\title{
Insect olfaction as an information filter for chemo-analytical applications
}

\author{
Dissertation
}

In partial fulfillment of the requirements for the degree of

Doctor of Philosophy (PhD)

of the Faculty of Forest Science and Forest Ecology

Georg-August-University Göttingen

\author{
Submitted by \\ Sebastian Paczkowski, \\ born in Berlin-Schöneberg \\ Göttingen, 2013
}


1. Reviewer: Prof. Dr. Stefan Schütz

2. Reviewer: Prof. Dr. Ursula Kües

Date of the oral examination: 29.05 .2013 


\section{Acknowledgements}

I like to thank all the people who advised and supported my work at the department of forest zoology and forest protection.

I also like to thank my supervisor, Prof. Dr. Stefan Schütz, for his support and advice.

I further like to thank Prof. Dr. Ursula Kües for her supervision of this thesis and Prof. Dr. Edmone Roffael for the evaluation of my oral examination.

I like to thank especially Dr. Bernhard Weissbecker and Dr. Gerrit Holighaus for their help and their critical opinions during so many discussions.

Further thanks go to all of the present and former staff of the department of forest zoology and forest protection. Thank you very much: Dr. Kai Füldner, Prof. Dr. Sergio Angeli, Evelin Kistner, Brunhilde Brunotte, Dr. Prodepran Thakeow, Dr. Bettina Johne, Julian Heiermann, Dr. Sonja Weißsteiner, Christine Rachow, Jan Seelig, Dr. Maximilian von Fragstein und Niemstorff, Dr. Martin Gabriel, Sara Nicke, Friederike Maibaum, Kira Duntemann, Ulrike Eisenwiener, Jörg Berger, Ullrich Aschmann, Reinhold Dankworth, Dr. Ferdinant Rühe, and Roswita Walbrecht.

I further like to thank all the external persons who supported my work: Prof. Dr. Claus-Dieter Kohl, Dr. Tilmann Sauerwald, Prof. Dr. Helmut Schmitz, Dr. Redelf Kraft, Dr. Jörg Gottschald, Moritz Essinger, Marco Bauer, Alexander Weiß, Daniel Meixner, Arnim Titze, Heino Schmeiß, Brian Inglis, and Prof. Dr. Barbara Wilson.

Further thanks go to all of the students who I supervised and who thereby contributed to my work.

I like to thank the Cusanuswerk, Bischöfliche Studienstiftung, for the financial support.

I like to thank my wife, Dr. Marta Paczkowska and my children Oliver Pavel, Isabella Jadwiga and Katharina Lotta for giving sense to my life. 


\section{Zusammenfassung}

Die Geruchswahrnehmung von Insekten ist an die spezifischen Anforderungen der Ökosysteme angepasst, in denen sie leben. Von Insekten wahrgenommene Volatile können Informationen über den Ort eines geeigneten Ovipositionsplatzes oder den physiologischen Zustand einer Wirtspflanze geben. Da der Geruchssinn der Insekten sich seit Millionen von Jahren an die Wahrnehmung bestimmter Volatile angepasst hat, können die wahrgenommenen Volatile als Markerstoffe angesehen werden, die zuverlässig einen Ökosystemzustand angeben können. Die Identifikation dieser Markerzustände und die Evaluation ihrer Nützlichkeit für spurenanalytische Anwendungen ist das Ziel dieser Arbeit.

Es wurden drei Themen ausgewählt um die Verwendungsmöglichkeit des Insektengeruchssinns für Sensoranwendungen zu überprüfen: Fleischfrische, Todeszeitbestimmung (post mortem Intervall, PMI) und frühzeitige Feuererkennung. Spurenanalytik (GC-MS), Elektrophysiologie (EAG, GC$M S / E A D)$, Verhaltensversuche und Feldtests wurden angewandt, um die chemoökologische Interaktion der Schmeißfliege Calliphora vicina und der „Feuerkäfer" Melanophila cuspidata, Merimna atrata and Acanthocnemus nigricans mit ihrer natürlichen Umgebung zu untersuchen. Die Resultate aus diesen Methoden erlaubten die Selektion von Volatilen, die drei Kriteria erfüllen: hohe Quantität, zuverlässige Emission und die ausschliessliche Emission von der untersuchten und keiner anderen Quelle. Diese drei Auswahlkriterien wurden aufgestellt um zu gewährleisten, dass die ausgewählten Markervolatile nicht nur zuverlässig mit dem physiologischen Status der Substrate (Alter von verderbendem Fleisch, PMI, Temperatur von erhitzten Spänen) korrelieren, sondern auch den Ansprüchen technischen Sensorsysteme genügen.

Im Falle des alternden Fleisches unter warmen und trockenen Bedingungen ist Nonanal ein Zeiger für die korrekte Reifung des Fleisches zu Schinken. Dimethyl Trisulfid, Phenol und Indol zeigen das Verderben des Fleisches unter warmen und feuchten Bedingungen an. Bei niedrigen Temperaturen sind zunehmende 2,3 Butandiol Emission und abnehmende Nonanal Emission ein Zeiger für zunehmendes Verderben des Fleisches, sowohl unter trockenen als auch feuchten Bedingungen. Allerdings wurde 2,3 Butandiol nicht von C. vicina wahrgenommen, da die Fliege während der Vegetationszeit aktiv ist und unter diesen Bedingungen Dimethyl Trisulfid, Phenol und Indol emittiert werden.

Nonanal, Hexanal, Dimethyl Disulfid, Dimethyl Trisulfid, Butan-1-ol und Phenol wurden als nützliche Volatile zur Eingrenzung der Leichenliegezeit ausgewählt. Die genannten Aldehyde sind typisch für die frühen Stadien der Verwesung, gefolgt von Butan-1-ol und den Schwefelsulfiden. Phenol wird hauptsächlich in den späteren Verwesungsstadien emittiert. Allerdings werden Phenol und 1-Butanol nicht von der Fliege wahrgenommen, da diese ein Generalist ist und nicht zwischen dem geblähten und aktivem Stadium der Verwesung unterscheiden muss. Daher wird zusätzlich die Geruchswahrnehmung des Speckkäfers Dermestes maculans betrachtet. Die Untersuchung anderer necrophager Insekten können weitere Einblicke in die Zeitabhängigkeit der Volatilemissionen während der Vertebratenverwesung geben. Die im Rahmen dieser Arbeit gewonnenen Ergebnisse 
erlauben eine Korrelation zwischen Emissionen und Leichenliegezeit und können eine Grundlage für eine volatilenbasierte post mortem Zeitbestimmung für Polizeikräfte sein.

Terpene, aliphatische Aldehyde, Furfural und Methoxyphenole werden von erhitztem Holz emittiert. Komponenten aus all diesen Stoffklassen werden von M. cuspidata und M. atrata wahrgenommen, da diese Käfer den Erhitzungszustand von Baumstämmen nach einem Feuer erkennen können, um einen geeigneten Ovipositionsplatz zu finden. A. nigricans nimmt vor allem Methoxyphenole wahr, wohingegen $M$. atrata und $M$. acuminata Furfural zur Orientierung verwenden. Diese Unterschiede komplementieren das bekannte Verhalten dieser Käfer in ihrer natürlichen Umgebung, da $A$.

nigricans auf verkohlten Stämmen, die große Mengen von Methoxyphenolen emittieren, seine Eier ablegt, und M. atrata und M. cuspidata auf Stämmen ihre Eier ablegen, die vom Feuer zwar äußerlich verkohlt, aber innerlich nur erhitzt wurden und daher Furfural emittieren. Halbleitergassensoren, die Furfural mit einer ausreichenden Selektivität vermessen können, können Holzbrände schon während ansteigender Temperatur und damit vor der Entzündung detektieren. Derartige Sensoren können sowohl als neuartige Frühwarnsysteme für die holzverarbeitende Industrie als auch für Waldbrandwarnsysteme oder den Haushalt dienen. 


\section{Abstract}

The olfaction of insect species is adapted to the requirements of the specific ecosystem they are living in. Volatiles perceived by insects can transport information on the location of an oviposition place or the physiological status of a host plant. As the olfaction of insects has adapted to the perception of distinct volatiles since millions of years, these volatiles can be regarded as marker compounds, which enable a reliable assessment of an ecological status. The identification of such marker compounds and the evaluation of their usefulness for trace analytical applications is the aim of this thesis.

Three topics were selected to evaluate the utilization of insect olfaction for sensor applications: Meat spoilage, post mortem interval (PMI) estimation, and the volatile based detection of wood fire before ignition. Trace analysis (GC-MS), electrophysiology (EAG, GC-MS/EAD), behavioral studies and field tests were used to understand the volatile based interaction of the blowfly Calliphora vicina and the fire beetles Melanophila cuspidata, Merimna atrata, and Acanthocnemus nigricans with their environment. The results gained by these methods allowed the selection of volatile marker compounds that fulfilled three criteria: high abundance, reliable emission, and unique emission from the substrate and not from any other sources. These criteria should ensure that the marker compounds's emission is not only reliably correlated to a physiological state of the substrate, e.g. an increasing spoilage of meat, a time point after death of a vertebrate, or a distinct temperature of wood prior to ignition, but that the marker compound can be sensed by technical sensor systems, as well.

In case of meat aging, the predominant emission of nonanal under warm conditions was an indicator for the correct maturation of the meat to ham. Dimethyl trisulfide, phenol, and indole indicated moisture induced spoilage under warm conditions. Under cold conditions, increasing 2,3 butanediol emission and decreasing nonanal emission was a spoilage indicator for both the dry and the wet samples. However, 2,3 butanediol was not perceived by the fly, because its activity is restricted to the warm vegetation period, where dimethyl trisulfide, phenol, and indole are emitted.

Nonanal, hexanal, dimethyl disulfide, dimethyl trisulide, butan-1-ol, and phenol are useful volatiles to estimate the PMI of vertebrates. The aldehydes are emitted in the early stages of vertebrate decay, followed by butan-1-ol and the sulfur compounds. Phenol is predominantly emitted in the later stages of decay. However, butan-1-ol and phenol are not perceived by the fly, which is a generalist among carrion visitors and does not differentiate between the early stage, the bloated stage, and the active stage of decay. The olfaction of other necrophagous insects, which prefer a distinct stage of decay, might lead to new insights into the time dependent volatile emission during vertebrate decay. The results gained by the investigation of the odor profile of decaying vertrebrates allowed a correlation between PMI and volatile profile that can be used by police forces as an additional indicator of the PMI. 
Terpenes, aliphatic aldehydes, furfural, and methoxyphenols are emitted from heated wood flakes. All of these compounds are perceived by M. cuspidata and M. atrata, as they can be used to assess the heating stage of a stem in order to find appropriate oviposition places. A. nigricans predominantly prefers methoxyphenols, while furfural is perceived by $M$. acuminata and M. atrata. These results complement the knowledge on the behavior of these beetles in their ecosystem, as $A$. nigricans oviposits on smoldering logs, which emit high abundances of different methoxyphenols, and $M$. atrata and M. cuspidata oviposit on heated but unburned stems, which emit, among other compounds, furfural. Semi conductor gas sensors perceiving furfural with a sufficient selectivity can predict a wood fire before it ignites. This would enable a new generation of early fire warning systems that can be used in the wood processing industry, wood land fire warning, or households.

These three examples show that insect olfaction can be utilized for the development of concepts for technical sensor applications, but that the ecological background of the insects has to be considered when selecting marker compounds. The restricted selectivity and sensitivity of technical sensors in comparison to the highly selective and sensititve biochemical perception mechanisms of insects have to be considered, as well. Therefore, the direct transfer from insect olfaction to technical solutions is not always possible. 


\section{Content}

1. Overview on the status of the manuscripts ............................................1

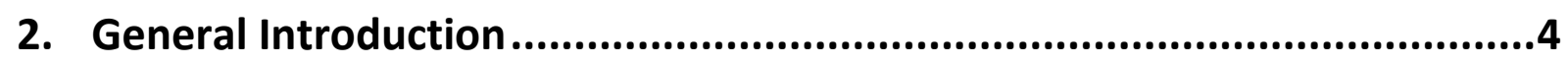

3. Biosensors on the basis of insect olfaction (Book chapter) ......................33

4. Post-mortem volatiles of vertebrate tissue (peer-reviewed mini-review) $\mathbf{5 0}$

5. Decaying mouse volatiles perceived by Calliphora vicina Rob.-Desv. (peerreviewed paper) ...............................................................................................70

6. Volatile emission of decomposing pig carcasses (Sus scrofa domesticus L.) as an indicator for the post mortem interval ................................................8

7. The olfaction of a fire beetle leads to new concepts for early fire warning

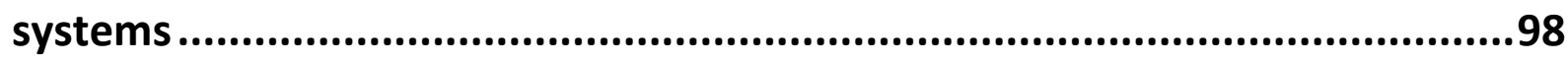

8. The olfaction of the fire beetle Merimna atrata as a model for early fire detection in eucalypt forests ................................................................116

9. Volatile combustion products of wood attract Acanthocnemus nigricans (Coleoptera: Acanthocnemidae). 129

10. Biomimetic gas sensors for large-scale drying of wood particles 142

11. Calliphora vicina (Rob.-Desv.) as an information filter for volatile-based meat spoilage detection

12. General Discussion 


\section{Overview on the status of the manuscripts}

Statement:

I hereby confirm that all of the corresponding authors/coauthors of the above listed publications agreed to be listed as coauthors for these publications.

2013

Paczkowski,S., Maibaum,F., Nicke,S., Ziegenhagen, H. and Schütz,S. (xxxx) Volatile emission of decomposing pig car-casses (Sus scrofa domesticus L.) as an indicator for the post mortem interval, Journal of Forensic Science, Manuscript in preparation

Author contributions:

Conceived and designed experiments: P.S., M.F., Z.H., S.S.

Trace analysis: P.S. (field sampling, GC-MS, statistics), M.F. (field sampling, GC-MS), Z.H. (field sampling, GC-MS)

Electrophysiology: P.S. (EAG, statistics), N.S. (EAG, statistics)

Wrote the paper: P.S., S.S., Z.H.

Paczkowski,S., Paczkowska,M., Nicke,S. and Schütz,S. (xxxx) The olfaction of the fire beetle Merimna atrata as a model for early fire detection in eucalypt forests, International Journal of Wild Fire, Manuscript in preparation

Author contributions:

Conceived and designed experiments: P.S., P.M., S.S.

Trace analysis: P.S. (thermal oxidation of wood chips, GC-MS, statistics)

Electrophysiology: P.S. (EAG, statistics), N.S. (EAG), P.M. (statistics)

Wrote the manuscript: P.S., S.S.

Paczkowski,S., Nicke,S. and Schütz,S. (xxxx) The olfaction of the blowfly Calliphora vicina (Rob.-Desv.) as an information filter for volatile-based meat spoilage detection. Meat Science, Manuscript in preparation

Author contributions:

Conceived and designed experiments: P.S., S.S.

Trace analysis: P.S. (meat spoilage, GC-MS, statistics)

Electrophysiology: P.S. (EAG, statistics), N.S. (EAG, statistics)

Wrote the manuscript: P.S., S.S.

2012

Paczkowski,S., Paczkowska,M., Dippel,S., Schulze,N., Schütz,S., Sauerwald,T., Weiss,A., Bauer,M., Gottschald,J. and Kohl,C.-D. (2013) The olfaction of a fire beetle leads to new 
concepts for early fire warning systems. Sensors and Actuators. In peer-revision, submitted 28.10.2012.

Author contributions:

Conceived and designed experiments: P.S., P.M., D.S., S.S., S.T., G.J., K.C-D

Trace analysis: P.S. (thermal oxidation of wood chips, GC-MS, statistics)

Electrophysiology: P.S. (EAD, EAG, statistics), N.S. (EAG, statistics)

Behavioral studies: P.S. (olfactometer, statistics), P.M. (olfactometer, statistics), S.N. (olfactometer, statistics), D.S. (olfactometer, statistics)

Sensor experiments: P.S. (data evaluation), S.T. (temperature cycle, surface development, gas mixer), W.A. (sensor surface development, gas mixer), B.M. (temperature cycles, burning chamber, portable MS), G.J. (sensor surface development, GC-MS/SOMMSA)

Wrote the paper: P.S., D.S., S.S., S.T., B.M., K.C-D

Paczkowski,S., Paczkowska,M., Dippel,S., Flematti,G. and Schütz,S. (2012) Volatile combustion products of wood attract Acanthocnemus nigricans. (Coleoptera: Acanthocnemidae) Journal of Insect Behavior, In peer-revision, submitted 30.11.2012.

Author contributions:

Conceived and designed experiments: P.S., P.M., D.S., S.S.

Behavioral studies: P.S. (olfactometer, statistics), P.M. (olfactometer, statistics), D.S. (olfactometer, statistics)

Wrote the paper: P.S., D.S., S.S.

Paczkowski,S., Maibaum,F., Paczkowska,M. and Schütz, S. (2012) Decaying mouse volatiles perceived by Calliphora vicina Rob.-Desv. Journal of Forensic Sciences, 57:1497-1506. Article was peer-reviewed.

Author contributions:

Conceived and designed experiments: P.S., M.F., P.M., S.S.

Trace analysis: P.S. (field sampling, GC-MS, statistics), M.F. (field sampling, GC-MS)

Electrophysiology: P.S. (EAG, statistics), P.M. (statistics)

Wrote the paper: P.S., M.F., S.S.

2011

Paczkowski,S., Sauerwald,T., Weiß,A., Bauer,M., Kohl,C.-D. and Schütz,S. (2011) Biomimetic gas sensors for large-scale drying of wood particles. Proceedings of the SPIE, 7975:797505797505-8. Not peer-reviewed.

Author contributions:

Conceived and designed experiments: P.S., S.S., S.T., K.C-D

Trace analysis: P.S. (thermal oxidation of wood chips, GC-MS, statistics)

Electrophysiology: P.S. (EAD, EAG, statistics)

Sensor experiments: S.T. (temperature cycle, surface development, gas mixer), W.A. (sensor surface development, gas mixer), B.M. (temperature cycles) 
Wrote the paper: S.S., P.S., S.T., B.M., K.C-D

Paczkowski,S. and Schütz,S. (2011) Post-mortem volatiles of vertebrate tissue. Applied Microbiology and Biotechnology, 91:917-935. Article was peer-reviewed.

Author contributions:

Wrote the paper: P.S. (concept, literature review, writing), S.S. (concept, writing)

Paczkowski,S., Weißbecker,B., Schöning,M.J. and Schütz,S. (2011) Biosensors on the basis of insect olfaction. In: Insect Biotechnology, Ed. Vilcinskas A, Springer, New York, p.225-240. Not peer-reviewed.

Author contributions:

Wrote the paper: P.S. (concept, literature review, writing), S.S. (concept, writing), W.B. (concept, literature review, writing), S.M.J. (concept, writing) 


\section{General Introduction}

Insects live on our planet since app. 360 million years. They can be found in nearly any ecosystem of the world and they are the class with the highest number of species in the animal kingdom. According to the laws of evolution, only the fittest survive (Darwin, 1859). The fittest individual in a population is not necessarily the strongest, but can be the one who shows the best adaption to the ecosystem it is living in. As conditions in ecosystems alter, the individuals with the highest flexibility to adapt to these changes are the fittest. In consequence, the wide-spread and large group of insects shows a high variation of successful strategies to fulfill the demands of various changing ecological niches (Grimaldi and Engel, 2005; Linksvayer et al., 2012).

Some of these niches require physical abilities, like flying or running, or abilities in orientation towards a food source or towards mating partners (Nation, 2001; Grimaldi and Engel, 2005). The basis for the orientation in complex environments is the selection of important stimuli. Such stimuli can be the optical impression of a host plant species (Briscoe and Chittka, 2001), the acoustic impression from a mating partner (Groot et al., 2010), the gustatory impression of a food source (Singh, 1998) or the volatile profile of a mating partner (Vogt and Riddiford, 1981). Among these senses, olfaction and gustation are the oldest senses in nature and especially olfaction plays an important role for the orientation of many insect species (Hansson, 1999). Insects can differentiate between healthy or damaged plants (Schütz and Weissbeker, 2003; Schütz et al., 2004; Schütz et al., 1997b) or can communicate with each other by means of olfaction (Pelosi and Maida, 1995).

Olfaction is based on the perception of volatile organic compounds (VOCs). In nature, VOCs are actively or passively produced as by- or end-products of chemical reactions (Schütz et al., 1997b; Paczkowski and Schütz, 2011). A plant can defend itself by the emission of volatiles (Feussner et al., 1997; Fineshi and Loreto, 2012) or an insect can attract mates by using its pheromone glands (Vogt and Riddiford, 1981). Draught stress (Schütz et al., 2004; Paczkowski et al., 2011b), auto oxidation (Choe and Min, 2005), or feeding by other insects (Schöning et al., 1998; Thakeow et al., 2008) are other examples for the origin of volatiles. On the other hand it has to be taken in account that olfaction in insects is not always an isolated impression. The process of finding a source can include other senses, as well (Schmitz et al., 2002; Heisswolf et al., 2007). An example for a possible interaction of senses, which can be connected to a sensitivation of sensory neurons, is the Australian little ashbeetle Acanthocnemus nigricans. The beetles are attracted by smoldering logs (Schmitz et al., 2002; Kreiss et al., 2007). They derive of an infrared sensitive organ for fire detection and use their olfaction to detect smoldering logs, as well (Schütz et al., 1999a). It is still a matter of discussion, if the beetles are activated by the scent of a fire and orientate towards it by means of their infrared detection (Evans, 2010). This could be an example of two complementary senses, as interactions between olfaction and other stimuli have already been observed (Heisswolf et al., 2007). These observations might contribute to recent discussions on the selectivity of insect olfaction (Hallem and Carlsson, 2006; Bohbot and Dickens, 2012). Odor cues are often compounds emitted by many different sources, which do not allow the exact identification of a single type of source, for instance a food source. Although pattern recognition is enabling insects to identify odor bouquets, additional senses will allow a more precise differentiation of the insect's environment. On the other hand, studies show the attractivity of isolated volatiles (Pelosi and Maida, 1995; Aak et al., 2010). 
The role of hormones and age in olfaction has been discussed, as well (Crnjar et al., 1990). It is known that neurosecretory cells (NSCs) in the protocerebrum of insects can release hormones, which are temporarily stable and can directly serve as neurotransmitters. Such neuropeptides can activate neurons over an intermediate time interval, causing a predisposition for detecting and corresponding to stimuli from the environment (Peric-Mataruga et al., 2001, Grimmekikhuijzen, 2012). These examples show that olfaction is influenced by the physiological state of the insect, as well.

All of these studies from the fields of evolution, physiology, genetics, biochemistry, behavior and chemistry of insects are interlinked and show that olfaction is based on complex interactions between an individual and its environment (Crnjar et al., 1990, Schütz et al., 1997b; Hansson, 1999; Schmitz et al., 2002; Schütz et al., 2003; Schütz and Weißbecker, 2003; Schütz et al., 2004; Heisswolf, 2007; Aak et al., 2010). In this thesis, these complex interactions were evaluated for applications in food science, forensic science, and fire control (Paczkowski et al., 2011a; Paczkowski et al., 2011b; Paczkowski and Schütz 2011; Paczkowski et al., 2012). Insects are adapted to the perception of volatiles that reliably convey information about the status of a host or the position of a mate. This adaption is the result of millions of years of evolutional pressure that favored the survival of the fittest (Darwin, 1859). According to volatile perception, the fittest individual is the one that perceives the volatiles allowing the most reliable identification of the source it is aiming to. Therefore, these volatiles can be regarded as pre-selected by nature and can be reliable markers for volatile based applications. In the frame of this doctoral thesis, the olfaction of the blowflies Calliphora vicina and $C$. vomitoria, as well as the olfaction of the Australian fire beetles Acanthocnemus nigricans and Merimna atrata and the European fire beetle Melanophila acuminata were investigated in order to identify reliable marker volatiles for meat spoilage detection, post mortem interval (PMI) estimation (Paczkowski et al., 2012) and early fire warning (Paczkowski et al., 2011a), respectively.

\subsection{Volatile organic compounds}

Volatile organic compounds (VOCs) are organic (= carbonic) compounds with a vapor pressure under SATP conditions (Standard Ambient Temperature and Pressure; $25^{\circ} \mathrm{C}$ and $1013 \mathrm{hPa}$ ) that is high enough to allow their evaporation in the gaseous phase. Although there is no uniform definition for VOCs, most approaches devide between very VOCs (VVOCs), VOCs, and semi VOCs (SVOCs), on the basis of their vapor pressure. However, there is no distinct definition at which vapor pressure or boiling point a volatile is classified as VVOC, SVOC, or VOC. While VVOCs, like $\mathrm{CO}_{2}, \mathrm{NO}_{x}$ or $\mathrm{CH}_{2} \mathrm{O}$, are completely in the gaseous phase under SATP conditions, SVOCs, like 3-methyl-2H-furo[2,3-c]pyran-2one, a compound that promotes seed germination after forest fires (Flematti et al., 2011) or methylbenzoate, a compound emitted by many molds (Parkinson et al., 2008), are evaporated at low concentrations.

VOCs are investigated in many branches of science. Especially in the atmospheric chemistry, VOCs are in the focus of recent research, because they can harm human health at high concentrations (Granström, 2002; Parkinson et al., 2008; Maleknia et al., 2009). Anthropogenic VOCs derive, for instance, from industry (Graedel, 1994), traffic ( $\mathrm{Na}, 2006)$, or consumers (Goldberg, 1993). In nature, VOCs can be by- or end-products of chemical reactions in plants (Feussner et al., 1997), animals (Paczkowski and Schütz, 2011), or fungi (Thakeow et al., 2008). The following subchapters give information on the formation and the physical properties of VOCs relevant for this thesis, as well as an overview on sampling procedures and identification methods. 


\subsubsection{Formation of VOCs}

\subsubsection{Wood}

Wood is a complex tissue consisting of hemicelluloses, cellulose, lignin, and a broad variety of accessory compounds. The relative amount of these constituents varies between tree species, within tree species and even within one tree (Kollmann, 1982). The most important group of volatile accessory compounds, especially in soft wood, is the group of terpenes (Granström, 2002; Banerjee, 2001; Englund and Nussbaum, 2000). At room temperature freshly felled wood emits terpenes with high vapor pressures (Englund and Nussbaum, 2000). Although this is a physical process, terpenes can transform to other compounds by chemical reactions (Neuenschwander et al., 2010). A second group of accessory compounds is the group of fatty acids. The parenchyma cells of wood contain fatty acids in the phospholipid membranes and in vesicles (Sjostrom 1993). Linoleic acid and arachidonic acid are typically abundant in these membranes (Frankel, 1983). Fatty acids can be degraded by autolysis, reactive oxygen species (ROS) (Choe and Min, 2005), thermal oxidation (Paczkowski et al., 2011a), and microbial enzymes (Paczkowski and Schütz, 2011). All of these processes might lead to the same volatile products. Autolysis or oxidation by ROS leads to the formation of the aldehydes hexanal, heptanal, nonanal and decanal (Risholm-Sudman et al., 1998; Svedberg et al., 2004; Arshadi, 2005; Roffael, 2006). However, aldehydes can form due to thermally induced oxidation (Arshadi, 2005) or fungal metabolism (Combet et al., 2006), as well. However, there is a lack of detailed studies on the formation of volatile lipid oxidation products (Combet et al., 2006; Kionka and Kunau, 1985; Heath et al., 2002).

Hemicellulose is a heteropolymer consisting of different hexose and pentose sugars. Together with cellulose, to which it is interconnected with pectin, it forms a net of fibers that makes the cell walls of plants more flexible (Nultsch, 2001). The most abundant sugars in hemicelluloses are the pentoses Dxylose and L-arabinose that can be polymerized together with glucose, xylose, mannose, galactose, rhamnose, mannuronic acid, galacturonic acid, and arabinose. These sugar monomers form, for instance, xylan, glucuronoxylan, arabinoxylan, glucomannan, and xyloglucan (Sjostrom, 1993). Cellulose is a macromolecule consisting of chains of biochemically polymerized glucose. The single glucose molecules are connected by 1,4- $\beta$-glycosidic bonds to the dimer cellobiose. In this subunit one glucose molecule is turned $180^{\circ}$. Repeatedly attached dimers form cellulose chains. These chains are organized in microfibers in which hydrogen bonds connect the chains to each other. These microfibers are integrated into the cell wall (Sjostrom, 1993; Nultsch 2001). Volatile products of hemicellulose combustion are acetic acid from the deacetylation of hemicelluloses, and hydroxyacetone and furanes from the degradation of pentoses and hexoses (Risholm-Sudman et al., 1998; Fengel and Wegener, 1984; Oasmaa et al., 2003; Tjeerdsma et al., 1998; Chheda et al., 2006; Roffael, 2006; Oasmaa et al., 2003).

Among the group of furanes, 2-pentylfurane, furfural and methylated furfural are reported in many of the published experiments (Risholm-Sudman et al., 1998; Fengel and Wegener, 1984; Oasmaa et al., 2003; Tjeerdsma et al., 1998; Chheda et al., 2006). Thermal degradation of the cellulose macromolecule leads to the cleavage of the 1,4- $\beta$-glycosidic bonds. Incomplete degradation yields, among many other products, hydroxyacetone (Piskorz et al., 1986; Zhang et al., 2008), 5methylfurfural (Oasmaa et al., 2003) and 3-methyl-2H-furo[2,3-c]pyran-2-one, also known as karrikinolide (Flematti et al., 2011) (Figure 1). 
A

\section{Biochemical}

Polymerization

B

Thermal

degradation

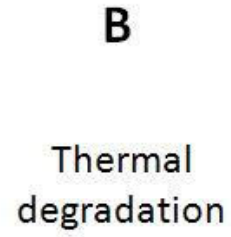

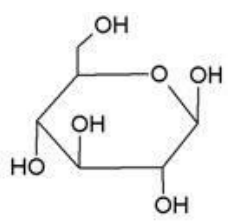

\section{D-Glucose}

C

5-Methylfurfural
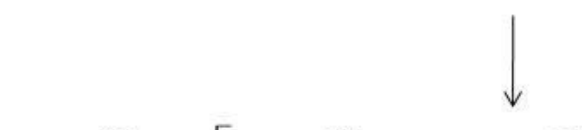

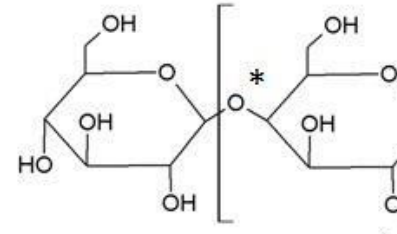

${ }^{*}$

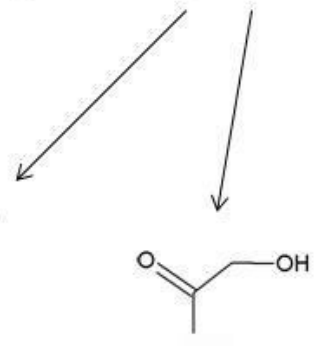

Hydroxyacetone
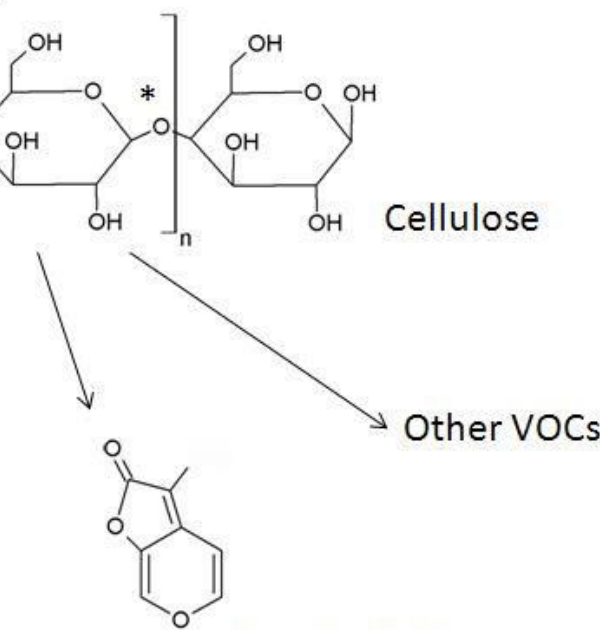

Karrikinolide

Figure 1 shows the structure of cellulose (B). The macromolecule forms from the biochemical polymerization of glucose (A). The smallest unit is the dimeric anhydrocellobiose, consisting of two glucose molecules. Glucose polymers assemble to microfibers, which are integrated in the cell wall. Incomplete thermal degradation yields hydroxyacetone, 5methylfurfural, 3-methyl-2H-furo[2,3-c]pyran-2-one (karrikinolide), and other volatile organic compounds (VOCs).

Lignin is, together with cellulose, the main compound of any wooden tissue. Although the complete structure of lignin is not fully understood, it is known that the bio-polymer lignin consists of several monomeric constituents. The lignin of hardwood is mainly made up of the two biochemically polymerized phenylpropanoids coniferylalcohol and sinapylalcohol, while softwood lignin consists of synapylalcohol (Sjostrom, 1993). The most prominent bond between two phenylpropanoids is the $\beta$ O-4-bond (Figure 2), but others also exist, for instance biphenyl- or $\beta$ - $\beta$-bonds exist (Higuchi, 1990). These are formed due to biochemical radicalization of sinapyl- or coniferylalcohol. Radicalization of the compounds leads to the formation of a polymerized lignin precursor (dimeric chinomethid). The following ionic addition reforms the aromatic center in the molecule. By repeating this process, more and more coniferyl- and sinapylalcohol units are added to the growing lignin molecule (Sjostrom, 1993, Higuchi 1990). An example for lignin depolymerization is the radicalization of lignin by UV-rays, resulting in bleaching of wood tissue because of the destruction of photoactive conjugated double bond systems, for instance in the delocalized $\pi$-electron systems of benzoic rings (Chang, 2001).

Temperature induced bond breaking of lignin is a physical influence that results in radicalization of molecules, as well. During a wood fire, heat and oxygen leads to a loss of electrons in the lignin molecule and consequently the bonds between the polymerized coniferyl and sinapyl components break, a process called oxidative cleavage (Gao et al., 2003). However, pyrolytic studies on wood prove the formation of the same volatiles under anaerobic conditions, which indicates oxidative processes without oxygen (Azeez et al., 2010; Branca et al., 2006; Ingram et al., 2008; Karagoz et al., 2005). 


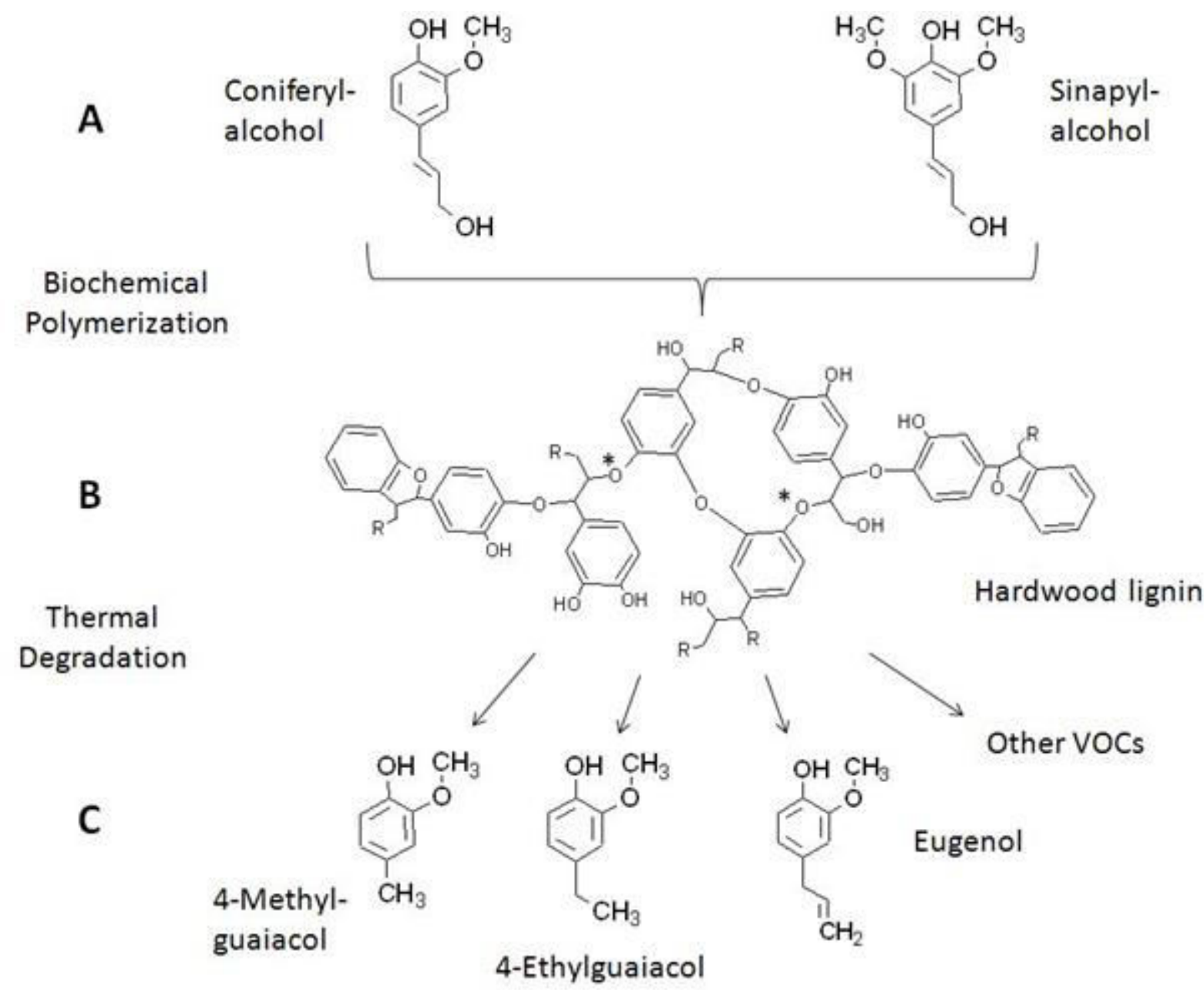

Figure 2 shows sinapylalcohol and coniferylalcohol (A), which are the basic compounds in hardwood lignin. Both compounds are polymerized by biochemical processes during the lignification of the cell wall. The result of this polymerization is the macromolecule lignin (B). $40-60 \%$ of the bonds between sinapyl- and coniferylalcohol are $\beta-0-4-$ bonds $(*)$. Thermal degradation of the lignin molecule yields the methoxyphenols 4-methylguaiacol, 4-ethylguaiacol and eugenol and other volatiles.

The released components are further oxidized to guaiacol derivatives, like 4-methylguaiacol, 4ethylguaiacol and eugenol, and a large number of other compounds (Figure 2), (Azeez et al., 2010; Branca et al., 2006; Ingram et al., 2008; Karagoz et al., 2005). A complete oxidation of lignin leads mainly to the formation of $\mathrm{CO}_{2}, \mathrm{H}_{2} \mathrm{O}$, and mineral containing ashes. Biochemical degradation of lignin can be induced by fungi (Oudia et al., 2008). White rot fungi derive of enzymes (phenoloxidases, peroxidases, chinon-reducing enzymes) that are able to oxidize polymerized coniferyl and sinapyl components by radical mediators (Mai et al., 2004). These processes can result in the formation of the same compounds that are formed by thermal degradation.

\subsubsection{Vertebrate tissue}

For specific details on the chemical composition and the degradation of vertebrate tissue, the reader is referred to the publication presented in chapter 3 of this thesis.

\subsubsection{Physics of VOCs}

Environmental factors are important for the formation, evaporation and distribution of volatiles. Physical parameters, such as temperature and wind speed are part of these environmental factors 
(Balkovsky and Shraiman, 2002). The temperature plays an important role in the ideal gas law, which explains the relation between pressure, volume, temperature, the mol of a gas, and the universal gas constant. The Maxwell-Boltzmann distribution derives from the ideal gas law and describes the mean kinetic energy of molecules in gases and fluids (Giancoli, 2010). This kinetic energy influences the rate of movement of the molecules. If this movement is fast enough, a molecule will have enough energy to overcome the binding forces to other molecules and can, for instance, evaporate from a fluid (Agafonova et al., 2012). The kinetic energy, which is sufficient for evaporation, is compound specific. Therefore, every pure compound has its specific boiling point, which is the temperature at which a pure compound completely evaporates. However, temperatures below the boiling point allow certain evaporation, as well, because few molecules, according to the Maxwell-Boltzmann distribution, have a kinetic energy higher than the averge (Giancoli, 2010; Zeng et al., 2010). This evaporation behavior is compound specific, as well, and is described by the vapor pressure of the compound (Giancoli, 2010; Agafonova et al., 2012). The vapor pressure is usually measured with the pure compound under SATP conditions, which means that the athmospheric pressure and the temperature are kept constant $\left(25^{\circ} \mathrm{C}, 1013 \mathrm{hPa}\right)$. If the specific vapor pressure of the pure compound and the athmospheric pressure are the same, the compound has reached its boiling point (Giancoli, 2010).

The vapor pressures of the pure compounds selected in the manuscripts presented in this study varied between 1 ppmv for 5-hydroxymethylfurfural and 14,500 ppmv for hexanal (Yaws, 2007; The good scents company, 2012). In order to relate the vapor pressure of the pure compound under SATP conditions to other concentrations, Henry`s law is the appropriate approach for diluted compounds at low concentrations (Pollien et al., 2003):

$$
p=k_{H} \times c
$$

where $p$ is the partial pressure of the solved compound in the gas above the solution, $c$ is the concentration of the compound in the solution and $k_{H}$ is the Henry's constant that depends on the solved compound, the temperature, and the solvent. $K_{\mathrm{H}}$ is experimentally determined. Henry's law takes in account the interactions of both temperature and solvent with the volatile compound. $A$ non-linear relation of $c$ and $p$ can be calculated by determining $k_{H}$ for different concentrations. As the Henry constants for most of the compounds used in this study was not known, Raoult's law was used (Stanford and Mcgorrin, 1994). Raoult's law implies a linear relation between the vapor pressure of a diluted compound and its concentration and has to be used for diluted compounds at high concentrations:

$$
p=p^{*} \times x
$$

where $p$ is the partial pressure of the solved compound in the gas above the solution, $p^{*}$ is the vapor pressure of the pure compound, and $x$ is the mole fraction of the solved compound. As the real vapor pressure / dilution curve is non-linear, Raoult's law is only an approximation of the real vapor pressure. Especially at low concentrations, the vapor pressure is underestimated.

Antoine 's law, which is a pragmatical simplification of the Clausius-Clapeyron equation (Manjare and Goshal, 2006), describes the relation between the vapor pressure and the temperature for pure compounds: 


$$
p=10^{A-\frac{B}{C+T}}
$$

where $p$ is the partial pressure of the pure compound in the gas above the solution, $T$ is the temperature, and $A, B$, and $C$ are experimentally determined constants.

Volatiles are usually emitted in plumes, which are distributed coincidentally in the environment. The combination of different wind speeds and wind directions lead to lamiar and turbulent wind currences which dilute the plumes and create a complex concentration gradient, which is not necessarily correlated to the position of the source (Villermaux and Innocenti, 1999; Fackrell and Robins, 1982). This makes it difficult to predict even the direction of a punctual volatile emission source (Balkovsky and Shraiman, 2002). Nevertheless, insects are able to select and approach a source by means of olfaction. Two different strategies allow the insect to find a source. The first strategy is an adaption towards the perception of volatiles at very low concentrations (Pelosi and Maida, 1995; Tegoni et al., 2004). The second strategy is the development of an effective searching behavior (Balkovsky and Shraiman, 2002).

Thus, the presented physical relations describing emission and distribution of volatile compounds show the complexity of the mere physical process. This complexity leads to an unpredictability of volatile emissions in a natural environment, which has to be considered especially when interpreting quantitative data on volatile compounds.

\subsubsection{Sampling of VOCs}

Sampling of VOCs is always restricted by the applied method (Rosa Ras et al., 2009). An important methodological factor is the sampling with static or dynamic head space. Static sampling is based on the passive collection of volatiles by an adsorbent placed near the emission source. Dynamic sampling is based on an airflow that sucks the emitted volatiles through the adsorbent. Another methodological difference exists between solvent free or solvent utilizing methods (Tholl et al., 2006).

As an example, the SPME (Solid Phase Micro Extraction) technique was developed for solvent free sampling in a static or dynamic headspace. A strong advantage of the method is the uncomplicated handling, but a disadvantage is the unprecise quantification. Details on SPME sampling are published in Paczkowski and Schütz, 2011. As this mini-review is already published and should not be modified, this cross reference should avoid unnecessary repetitions. For more information on SPME sampling see Tholl et al., (2006) and Wardencki et al., (2004).

Another volatile collecting method is the solvent based closed loop stripping apparatus (CLSA) method for dynamic sampling, firstly applied by Grob in 1976 (Grob and Zürcher, 1976). A pump is circulating air in a closed system and a charcoal filter is collecting the volatiles emitted by the sample. CLSA filter tubes are manufactured with an exact weight of charcoal fixed by a metal wire in a glass tube. After collection, the volatiles in the charcoal filter are eluted with a solvent (Hwang, 1984; Osemwengie and Steinberg, 2003; Nawrath et al., 2012). This is the advantage of this method, because the eluat can be analyzed several times by different analytical methods in order to confirm the identity of the compounds. Another possibility is the repeated analysis by the same method in order to estimate the standard deviation of the analysis. The eluat can be stored at low temperature. Major drawbacks of the method are the complex sample preparation in comparison to the TDS or 
SPME method, which can lead to contaminations (Tholl et al., 2006). Furthermore, the dilution of the collected volatiles during eluation can dilute a compound's concentration below the detection limit. Adapting the amount of solvent can improve this effect. Another disadvantage is the injection of the solvent into the GC, which covers many highly volatile compounds in the large solvent peak. This disables the identification of highly volatile compounds (Paczkowski and Schütz, 2011), which are detectable by the solvent free methods SPME and TDS.

The TDS sampling method (Thermal Desorption System) is based on a powder fixed in a glass tube by metal wire caps. There are different powders (Tenax, Carbotrap, Carbosieve, Carboxen or others) with different adsorption properties (Rothweiler et al., 1991), which can be combined with each other in one glass tube (Vass et al., 2004; Vass et al., 2008). The air is actively sucked through the tube during sampling and the volatiles are adsorbed on the powder. After sampling, the volatiles are thermally desorbed by an increasing temperature and a constant air flow leads them to a cooling trap. The advantages of the TDS method are the good sensitivity and the uncomplicated handling, which decreases the possibility of contaminations. Furthermore, the sampling method does not require a solvent and allows accurate quantification. On the other hand, the sample can be analyzed only one time and the equipment for TDS sampling and sample injection is expensive in comparison to other methods. Furthermore, a high amount of water vapour in the sampled air can cover the presence of VOCs at the retention time range of water. The selection of the appropriate adsorbent can decrease this problem (Helmig and Vierling, 1995).

The direct headspace method comprises several approaches for the sampling of a gas volume and its injection in a GC. One variation is that containers under vacuum suck the air from the environment and can be closed and transported to the lab. In the lab, gas samples can be taken with syringes from the container and can be injected in a cool injector of a GC. An easier method is the storage of a sample in a glass vial and the direct injection of the head space above the sample in the vial into the cool injector by an auto sampler (Hübschmann, 2001). In general, direct headspace sampling has the strong advantage that it is relatively unselective because the kind of volatiles and their quantities are not influenced by selective adsorbents. Therefore, the injected gas volume resembles the original composition of the sampled gas. Furthermore, contaminations due to sample processing are minimized. A major drawback is the low concentration of the volatiles, which are not enriched on an adsorbent (Tholl et al., 2006). The only possibility to avoid concentrations below the detection limit of a detector is the injection of a large gas volume, which is problematic because of the water content of the sample volume that leads to column bleeding (Helmig and Vierling, 1995).

The last sampling methods described here is the trapping of the volatiles by leading them through cooled liquid solvents, e.g. ethanol, cyclohexane, or dichloromethane (Dufour et al., 2007) or extracting them in these solvents (Thakeow et al., 2008). This method has the advantage that it results in large quantities of volatile containing solvent, which can be further analyzed even by bench-chemistry methods. Of course, the method is not useful for low emission rates, because usually only low amounts of the volatiles diffuse from the gaseous to the liquid phase. Most of the published work on wood pyrolysis uses this method, because it is useful for sampling high emission rates. Sensitive methods, for instance TDS or SPME, will adsorb too many volatiles and will cause an overloading of the column, which results in insufficient peak separation, distorted peaks and restrictions in quantification (Hübschmann 2001). 


\subsubsection{Identification of VOCs}

The identification of volatile organic compounds covers simple bench-chemistry methods, which use an indicator that reacts in presence of a compound or a group of compounds (Hayes, 2004), fast analytics by infrared or UV desorption (Didomenico and Rendhal, 2004) and more complex analytical devices (Gutmacher, 2011; Merford, 2002; Kim et al., 2011). If the formation process of a compound is understood and if this process results in one or few products, the identification of this compound can be done by simple methods, sometimes even by gas sensor devices (Waser, 2003; Paczkowski et al., 2011a; Kim et al., 2011). However, in order to understand this formation process and to calibrate a simpler detection method, it is necessary to apply more complex identification methods. There exist various analytic devices for broad scale identification of volatile compounds (Merfort, 2002). In the frame of this doctoral thesis, gas chromatographs (GCs) were used for volatile sample processing and three different detectors (FID, MS, and EAD) analyzed the compounds in the sample. For more detailed information on gaschromatography see Hübschmann (2001) or Weißbecker et al., (2004).

\subsubsection{Injector}

The type of injector depends on the volatile sampling method. A TDS system requires a thermo desorption unit in order to desorb the volatiles from the adsorption powder with a temperature program $\left(40^{\circ} \mathrm{C}\right.$ to $250^{\circ} \mathrm{C}$ ) (Tholl et al., 2006; Vass et al., 2004; Vass et al., 2008). The desorbed volatiles are transported by a gas flow (usually helium) to a cryo trap, were they freeze $\left(-80{ }^{\circ} \mathrm{C}\right)$ before the resulting mixture is heated for further transport to the column. This is of high importance, because the following separation requires a fixed starting time for the column's temperature program (Hübschmann, 2001). A cryo injection port is also vital for direct headspace methods, were the injected gas has to freeze for fixation. Volatiles on a SPME fiber can be adsorbed in a conventional injection port at high temperatures $\left(250^{\circ} \mathrm{C}\right)$ and the compounds are fixed at lower temperatures at the beginning of the column (Wardencki et al., 2004). A liquid sample can directly be injected in a heated $\left(250^{\circ} \mathrm{C}\right)$ standard injector (Thakeow et al., 2008).

The injection mode of an injector can be splitless, meaning that the complete volume of the sample reaches the column, or can have a split ratio, meaning that only parts of the injected sample are led to the column. The split mode is useful when a sample has a high concentration of volatiles and overloading of the column should be avoided (Statheropoulos et al., 2005). As described above, overloading can cause restrictions in qualitative and quantitative analysis (Hübschmann, 2001).

On-column injection requires a low concentration in the sample, as the sample cannot be diluted by a split. As the sample is not heated in the injector, no thermally induced chemical reactions take place. The focusing can be enhanced by a cooling trap (Hübschmann, 2001).

\subsubsection{Column}

The volatile compounds are led through the column in the mobile phase by a carrier gas, which is usually inert helium. The interaction of the volatiles with the stationary phase depends on the vapor pressure of the compound, both the chemical characteristics of the compound and the stationary phase and the temperature (McNair and Miller, 1997). For instance, a polar column has a higher retention for polar compounds. However, there exist many different columns, which vary not only in the chemical composition of the stationary phase, but also in their length (3-60 m), their inner diameter (0.05-0.53 mm), and their stationary phase thickness (0.1-5.0 $\mu \mathrm{m}$ ) (Hübschmann, 2001; 
Poole, 2012). The influence of all of these factors on the separation performance is described by the distribution coefficient of a compound (McNair and Miller, 1997; Hübschmann, 2001). For instance, a distribution coefficient of 2 means that the compound is solved twice as much in the stationary phase than in the mobile phase. A distribution coefficient of 1 means that a compound is solved to the same extent in the mobile and the stationary phase and a coefficient of 0 means that the compound is not solved in the liquid phase, which resembles the behavior of pure air (McNair and Miller, 1997).

An equation with the most important factors influencing the resolution of a GC column is shown here:

$$
R_{S}=\frac{\sqrt{N}}{4}\left(\frac{k}{k+1}\right)\left(\frac{\alpha-1}{\alpha}\right)
$$

where $R_{S}$ is the resolution of the GC run, $N$ is the efficiency as a function of the type of gas, length of the column and inner diameter of the column, $k$ is the retention as a function of temperature, stationary phase thickness, and inner diameter of the column and $\alpha$ is the selectivity as a function of the temperature and the type of the stationary phase. The temperature of the column and the kind of stationary phase are the most important factors influencing $R_{S}$ (Harris and Habgood, 1966). Hower, all factors have to be combined carefully in order to get a performance adapted to the requirements of the analysis and most often the maximum resolution is to costly or one GC run takes to much time to enable efficient work in analytical labs (Hübschmann, 2001; Poole, 2012, McNair and Miller, 1997). For instance, under isothermal conditions the doubling of the column lengths increases the resolution with a factor of $1.4(+40 \%)$, while the time of the analysis is doubled $(+100 \%)$ and the column is more costly (McNair and Miller, 1997). In the food industry such a solution might cause trouble in the lab organization, because many samples have to be analyzed, while in research institutes a high resolution can be crucial. In this doctoral thesis the type of the stationary phase was the most important factor that influenced the resolution of the analysis, while the other factors were kept constant for most of the analytical experiments.

Under constant analytical parameters, the compound specific retention allows the identification of a compound, because a standard solution with this compound can confirm its identity by matching the retention time. However, several compounds can have the same retention time even under constant analytical parameters. Therefore, further confirmation of the compounds identity, for instance by a mass spectrometer, is usually necessary. If the standard is run with different GC parameters, the retention index can be calculated by means of a reference mixture, for instance alkanes with different chain length (Castello et al., 2009).

\subsubsection{Detector}

The compounds are detected after leaving the column. Different detectors exist for different purposes. There are detectors that analyze only parts of the sample, for instance, only sulfur and phosphor containing compounds (FPD: Flame Photometric Detector) (Berijani et al., 2006) or nitrogen and phosphor containing compounds (NPD: Nitrogen Phosphorous Detector) (Dorea et al., 1996). 
In a FID (Flame lonization Detector), all compounds of a sample are burned in a hydrogen-air flame after the separation on the column. During this burning, ions are emitted, which hit on a detector plate. The detector plate changes its resistance due to the contact with the electrons. The intensity of this change is defined by the quantity and the structure of the compound (Van Hamme et al., 2000). Therefore, the resulting peak area corresponds to the amount of the compound in the sample. The chromatogram shows large and small peaks at compound specific retention times. The only possibility of identifying a compound is the injection of a standard compound, which is analyzed with the method of the sample. If the standard compound has the same retention time as the compound in the sample, it is very probable that its identity is the same (Hübschmann, 2001).

A quadrupole mass spectrometer (MS) is one of the most popular detectors for GCs. It identifies compounds by their specific fragmentation pattern and not only by their retention time. In order to fragment compounds from a GC column, the compounds are led into a vacuum and through an electronic beam. This beam is emitted by a filament under a defined voltage, usually $70 \mathrm{eV}$. The electrons in the beam hit electrons out of the molecules, which leads to the formation of ionized fragments with a positive charge. These fragments are repelled by an electrode with positive charge and focused by two electro-magnetic lenses. The fragments reach the quadrupole, which consists of four rods under an oscillating voltage, which induces an oscillating electromagnetic field. The penetrability for any fragment is determined by the frequency of the electromagnetic field. A fragment can pass only if the frequency corresponds to its mass/charge ratio. The fragments passing the quadrupole hit a detector plate and can be assigned to their mass. From a statistical point of view the formation of fragments with different masses depend on probabilities. Therefore, each compound has its fragment pattern with a compound specific relation between fragments that form with a high probability and fragments that form with a lower probability. This fragment pattern is called mass spectrum and can be compared to mass spectra of known compounds (Hübschmann, 2001). A high analogy of the measured spectrum with a spectrum from a database indicates the identity of the compound in the sample (Watson et al., 2004). Running a purchased standard compound with the same analytic parameters can further confirm the identity of the compound by the retention time. Details and limitations of compound identification by GC-MS are given in the gereral discussion of this thesis.

After the identification of a compound it can be quantified by analyzing a standard sample with known compound concentration and comparing the standard peak area to the sample peak area. As performing a reliable quantification is rather complicated, this topic is referred to in detail in the general discussion. In general, the accuracy of the quantification influences the informative value of the results, which have to be discussed concerning this restriction (see "Formation of VOCs" and "Physics of VOCs" in the general introduction).

\subsection{Insect olfaction}

The sense of olfaction is essential for many insect species, as they use it for basic processes in their biology, such as mating (Pelosi and Maida, 1995), oviposition (Verheggen et al., 2008), finding food resources (Beck et al., 2012), and interspecific communication (Reyes-Vidal, 2009). There are manifold different semio-chemicals which are used by insects to serve these purposes. These semiochemicals are classified in inter- and intraspecific semio-chemicals. Interspecific semiochemicals (allelochemicals) are used for the communication between species and interspecific semio-chemicals 
(pheromones) are used for the communication within species (Schaefer, 2003). Pheromones are known for their role in sexual communication, for instance in the lepidopteran species Bombyx mori (Maida et al., 1993; Sakurai et al., 2004) or Lymantria dispar (Plettner et al., 2000). But pheromones can also serve different purposes, for instance aggregation on appropriate food sources (Gruter et al., 2012), alarming of a group (Vandermoten et al., 2012), and marking of oviposition places (Nufio and Papaj, 2001). They can be actively released from thoracic or abdominal glands by the sender (Blomquist and Vogt, 2003). Although there are different definitions (Ruther, 2002), allelochemicals can be classified by the type of relation between sender and receiver. There are kairomones, which are advantageous for the receiver and allomones, which are advantageous for the sender (Burger, 1988; Ruther, 2002; Schaefer, 2003). An aggregation pheromone can attract predators and is a kairomone, while a compound serving for predator defense is an allomone. Synomones are both advantageous for the sender and the receiver (Shepherd, 2007). Synomones can mediate avoidance of competition between two species. Apneumones are released by organic matter and are advantageous for one species, but disadvantageous for another species. An example is the attraction of a food source for a species, which also attracts predators of this species (Aak et al., 2010). Besides these classical definitions there are many other more specific definitions, and sometimes it is hard to define the class of a volatile because of its complex function in an eco-system (Nordlund et al., 1981).

Most of the listed chemo-ecological relations rely on the detection of single compounds rather than compound groups. The ability to select and sensitively sense single volatile compounds from complex odor patterns in a habitat requires precise sensory organs and highly developed information processing (Blomquist and Vogt, 2003). On the other hand, there are many examples for a broad scale tuning in insect olfaction, as well, suggesting that both approaches make sense for insects (Hallem and Carlsson, 2006; Bohbot and Dickens, 2012).

For specific details on the biochemistry of insect olfaction the reader is referred to the published book chapter presented in chapter 3 of this thesis. For more details see Hansson (1999), Pelosi and Maida (1995), Tegoni et al., (2004), Wicher et al., (2008), Hallem and Carlsson (2006) and Bohbot and Dickens (2012).

\subsection{Electrophysiology}

In an olfactory neuron, an odor induces a change of the dendritic membrane potential. This membrane potential is a product of different ion concentrations inside and outside of the neuron. When the dendritic membrane potential changes a neuron can be depolarized over the threshold for excitation and the potential change is transmitted along the axon-membrane of the neuron. The altering electrical potential of an axon can be measured by two electrodes, one inside the axon and one outside, as a reference electrode. (Schneider, 1957; Satelle et al., 1980; Schmidt et al., 2011). There exist different electrophysiological setups that apply the described biophysical relation. Single sensillum recording (SSR) utilizes single olfactory neurons for signal evaluation (Van der Pers and Minks, 2011; Shanbhag et al., 1999). While the reference electrode can be situated in the head of an insect, the measuring electrode is inserted in the axon of an olfactory neuron. This electrophysiological method requires a lot of skill and patience, as the sensillae on the antennae cannot be seen with a binocular. Therefore, the only reference for a successful insertion of the electrode is the measuring of an action potential rate (Lewicki, 1998). The advantage of this method is the possibility to classify different types of sensillae, which can result in a mapping of an antenna. 
Among the most popular examples of the classification and spatial allocation of sensilla populations is a map of the funiculus of Drosophila melanogaster (Shanbhag et al., 1999). The classification of a sensilla population's odor spectrum is important for the understanding of the mechanisms of olfaction in insects (Kaib, 1974) and research on this field was crucial for developing hypotheses for odor perception in insects (Hallem and Carlson, 2006; Bhobot and Dickens, 2012). The electrophysiological investigations for the presented doctoral thesis were performed with a different electrophysiological setup, called electroantennogramm (EAG).

\subsection{Electroantennogramm (EAG)}

While single sensillum recording measures the performance of one olfactory neuron, the electroantennogramm measures the depolarization of sensilla groups on the whole antenna. This can be done by clamping the excised antennae between two electrodes in an antenna holder. Usually, an electrolytic solution connects the antenna with the electrodes. An odor stimulus on the antenna leads to action potentials in all of the olfactory neurons of one sensilla population, which results in a depolarization of the whole antennae. This depolarization is caused by an increasing influx of positively charged ions into the neurons. The increasing negative charge in the surrounding cell lymph of the neurons leads to a measurable change in the voltage between two electrodes. This voltage change is amplified by a pre-amplifier and a main-amplifier. A low-pass filter is used to suppress the influence of drifts and a high-pass filter is used to suppress the influence of high frequencies of action potentials. The resulting signals show peaks that are correlated to the presence and the concentration of volatile stimuli (Weissbecker et al., 2004; Sauer et al., 1992; Schütz et al., 1999b).

In order to apply this stimulus, the volatile can be diluted in paraffin oil and this solution is dropped on filter paper and inserted in a syringe with a defined volume (Weissbecker et al., 2002; Paczkowski et al., 2012; Schütz et al., 1999b). For ideal solutions, the equilibrium concentration of the volatile stimulus in the defined volume of the syringe corresponds to its vapor pressure (Henrie's law) and depends on its concentration (Henrie's law or Raoult's law) and the temperature (Antoine's law). When the compound in the syringe has reached its equilibrium concentration, a defined amount of the gas volume is puffed on the antenna in the antenna holder. With this technique, dose response curves can be recorded that show the sensitivity of the antenna towards a stimulus in comparison to a control stimulus (Schütz et al., 1999b; Paczkowski et al., 2012). With a statistical analysis, the lowest significant response towards the volatile stimulus can be calculated. The vapor pressure of this lowest significant response depends on the vapor pressure of the pure compound and the concentration of the compound (Raoult's law or Henrie's law) and can be calculated in order to compare the dose-response relation between volatile stimuli with different vapor pressure.

A modified electrophysiological method based on the EAG method is the electroantennographic detection. The EAG setup is connected as a detector to a gas chromatograph (Weissbecker et al., 2000; Smid et al., 2002). This electroantennographic detection can be combined with a MS analysis (GC-MS/EAD) (Weissbecker et al., 2004; Riolo et al., 2012). At the end of the column of a GC a split can direct one part of the gas flow to the mass spectrometer and another part to the EAG setup as a second detector. Both the ion chromatogram of the MS and the electrophysiological chromatogram of the EAD can be combined in order to identify a volatile and classify it as electrophysiological active or inactive. 


\subsection{Applications of insect olfaction}

As the formation, sampling, and detection of VOCs, as well as the capabilities of insect olfaction and its application in trace analytical methods has been introduced in the previous subchapters of the introduction, the basic hypothesis of this thesis will be introduced here. This hypothesis regards the olfaction of insects as a highly promising model for portable trace analytical devices, such as gas sensors, designed for industrial applications (Vass et al., 2002; Statheropoulos and Karma, 2005; LeBlanc and Logan, 2010; Paczkowski et al., 2011a; Paczkowski et al., 2011b; Paczkowski and Schütz, 2011; Paczkowski et al., 2012). The cause for posting such a hypothesis was the common problem that has to be faced when adapting gas sensors for the detection of process-correlated volatiles: the problem of the choice of the volatiles that reliably indicate the incident that has to be detected (Winquist et al., 1993). Finding such a volatile can be highly valuable, because a sensor system that detects this volatile will sense an incident with high reliability and, in consequence, can be a commercializable innovation. Insect olfaction can be highly beneficial for selecting such volatiles, because insects are already adapted to perceive them (LeBlanc and Logan, 2010).

As chemosensing is one of the oldest senses in nature, million years of evolution have developed a complex interaction of odorant binding proteins, membrane receptors, and neurons in recent insects (Hansson, 1999; Tegoni, 2004; Hallem and Carlsson, 2006; Bohbot and Dickens, 2012). Such a long time periode was necessary, because most mutations leading to alterations in the DNA are nonsense mutations and the phaenotype of the individuals carrying such mutations is not affected. Even most of the mutations that make sense and lead to a change in the phaenotype of the individual are not located on sequences expressed for the sense of olfaction. If they are located there, it will be only a small part of these mutations that improve the abilities of the individual for the interaction with its changing environment. These small number of mutations will improve the individual's fitness in the struggle for live. The resulting phaenotypical changes will alter the population, as the individual with the best adaption will increase its chance to survive and to reproduce (Darwin, 1859) and, therefore, to disperse its genes, which contain the mutation (Storch et al., 2001). This basic description of evolutional processes shows the low probability for advantageous modifications in genes and underlines the importance of a long time and a high number of individuals necessary to generate such complex biochemical interactions in the olfaction of recent insect species (Pelosi and Maida, 1995; Tegoni et al., 2004; Wicher et al., 2008; Hallem and Carlsson, 2006; Bohbot and Dickens, 2012). These laws of evolution support the selection of reliably occuring volatiles, because part of an individual's fitness is its efficiency in detecting, for instance, a food source, a mating partner or an oviposition place. This detection process is often triggered by volatiles, because volatiles allow an assessment of the physiological stage of a substrate, which can be crucial for oviposition or feeding (Schütz and Weißbecker, 2003; Schütz et al., 2004; Schütz et al., 1997b; Schütz et al., 1999a; Thakeow et al., 2008). Using such volatiles for sensor solutions can reduce the time and the costs for fundamental research activities and can efficiently transfer fundamental biological knowledge to applied technical innovations (Paczkowski et al., 2011a; Schütz et al., 1997a; Schöning et al., 1998; Paczkowski et al., 2011b; Schütz et al., 1999a). This hypothesis has been evaluated in the frame of this thesis on the three examples meat spoilage, vertebrate decomposition, and thermal oxidation of wood. In case of meat spoilage, the olfaction of the blowfly C. vicina has been investigated (Paczkowski et al., 2012) and in case of the thermal oxidation of wood, the pyrophilic beetles $M$. atrata, M. acuminata and A. nigricans were investigated (Paczkowski et al., 2011a). 
A more detailed description of the different ideas concerning applications of insect olfaction are included in the book chapter "Biosensors on the basis of insect olfaction", which is part of this thesis (Paczkowski et al., 2011b). As this book chapter is already published and should not be modified, this cross reference should avoid unnecessary repetitions. The limits of the hypothesis are discussed in the general discussion of this thesis.

\subsection{Semi conductor gas sensors}

Semi conductor gas sensors are used for the detection of organic and inorganic gases (Winquist et al., 1993; Bock et al., 1997; Kohl, 2003; Kuske, 2006; Rajamaki et al., 2006; Olafsdottir and Kristbergsson, 2006, Paczkowski et al., 2011a, Eßinger et al., 2010). According to the quantum theory, electrons in a metal crystal are regarded as an electron gas. The kinetic energy of these electrons is described by the Fermi-Dirac statistic, which is an adaption of the Maxwell-Boltzmann statistic for quantum mechanical systems. The Fermi-Dirac statistic takes in account the Pauli Principle, which demands that every electron in a molecule must have a different quantum number. The band-model for crystals was developed on the basis of the Schrödinger equation and postulates that a metal crystal derives of energy bands. Within the band the possible energy state of electrons seems to be continuously, because of the high number of discrete energy states for the electrons. Between the bands there are band gaps without electrons. In conductors, the valence band is the conduction band, because the valence band has free energy states for stimulated electrons. An isolator has a very large gap between the filled valence band and the empty conduction band and stimulated electrons cannot pass to the conduction band. A semi conductor has a relatively small gap between the filled valence band and the conduction band. This small valence-conduction band gap leads to the typical electrical properties of semi conductors, which can be used for gas detection (for more information see Giancoli, 2010).

On the surface of a heated metal oxide crystal, volatiles can be oxidized by the oxygen or can reduce the oxygen in the metal oxide. This influences the energy states in the band, which leads to a band bending of the valence band and, in consequence, to a change in conductance of the semi conductor metal oxide crystal (Kohl, 1989; Madou and Morrison, 1989). This altering conductance can be measured and results in a signal allowing an estimation of the quantity and the type of volatile. The sensitivity and selectivity of such gas sensors can be modified by the nano structure of the surface, the type of metal oxide or other substrates, such as porphyrins or copper-phthalocyanines, and temperature or voltage cycles (Sauerwald et al., 2007; Gramm and Schütze, 2003).

Gas sensors are regarded as good candidates for many applications, as they derive of several advantageous features (Gutmacher et al., 2011). They allow a precise online monitoring of gas emissions, as they can measure volatiles every second and over a period of several years. They can be produced with relatively low costs and are small. Gas sensors can detect low concentrations of volatiles; however, major drawbacks are the relatively high energy consumption that disables standalone solutions (Gutmacher et al., 2011) and the lack of selectivity (Semancik et al., 2001, Gramm and Schütze, 2003). In a complex odor plume there might be important marker volatiles in traces. A compound that quantitatively dominates the odor profile might induce a strong change in conductance on the semi conductor gas sensor, which covers the smaller change in conductance induced by the marker volatile. While insects use their biochemical olfaction to select compounds from an odor mixture (Tegoni et al., 2004; Hallem and Carlsson, 2006, Wicher et al., 2008; Bohbet 
and Dickens, 2012), gas sensors cannot reach this level of selectivity (Kuske et al., 2006; Olafsdottir and Kristbergsson, 2006). Besides optimization of a single gas sensor`s selectivity, arrays with signal integration can be used to enhance selectivity (Gutmacher et al., 2011). In the frame of cooperation with the Institute of Applied Physics in Gießen, Germany, a first step was made in the development of an early fire warning system for wood flake dryers in the wood processing industry, which is basically transferable to forests or wood storages. The sensors in this multi-sensor array are run with a temperature cycle that enhances their selectivity (Paczkowski,S., Paczkowska,M., Nicke,S., Schulze,N., Dippel,S., Schütz,S., Sauerwald,T., Weiss,A., Bauer,M., Gottschald,J. and Kohl,C-D (xxxx) The olfaction of a fire beetle leads to new concepts for early fire warning systems, Sensors and Actuators B-Chemical, Manuscript in preparation).

\subsection{Scope of this thesis}

The scope of this thesis is the utilization of insect olfaction for three fields of application: forensic science, fire control, and food science. The first chapter "Biosensors on the basis of Insect olfaction" introduces the general idea of biosensors and biomimetic sensors.

The following mini-review and two experimental studies show the possibilities of using necrophageous insects, mainly $C$. vicina, as an information filter for identifying marker compounds that can be correlated to the post mortem time. The mini-review "Post-mortem volatiles of vertebrate tissue" introduces to the general idea and shows the complexity of volatile formation and volatile pattern published in the literature. The first experimental study "Decaying mouse volatiles perceived by Calliphora vicina Rob.-Desv." shows the preliminary results with mice as model organisms. A correlation between the calculated ADD and two volatile compounds is evaluated. The second study uses pigs as a larger model organism resembling human corpses, and yields more marker volatiles.

The following four manuscripts evaluate the use of the olfaction of fire associated beetles on the field of fire warning. The first manuscript "The olfaction of a fire beetle leads to new concepts of fire warning systems" evaluates the olfaction of the European fire beetle Melanophila acuminata for use in gas sensor based fire warning systems. The second manuscript uses the Australian fire beetle Merimna atrata to compare fire beetles in different habitats and, thereby, confirming the hypothesis of marker volatiles for wood fires, as there should be parallels between the olfaction of both beetles if evolution really tunes the olfaction to the perception of the most reliable compounds. The third manuscript "Volatile combustion products of wood attract Acanthocnemus nigricans (Coleoptera: Acanthocnemidae)" investigates the olfaction of a second Australian fire beetle with behavioral studies, as it was not possible to use electrophysiological methods with this animal. This study confirms the importance of lignin and cellulose derived combustion products, which also play a role in the other two manuscripts. The last manuscript "Biomimetic gas sensors for large scale drying of wood" focusses on presenting the general idea of the second part of this thesis for a conference (SPIE 2011). Here, the applicability of the hypothesis is emphasized.

The last manuscript "The olfaction of the blowfly Calliphora vicina (Rob.-Desv.) as an information filter for volatile-based meat spoilage detection" transfers the idea of post mortem time determination to meet spoilage. Again, $C$. vicina is used as an information filter for the identification of marker volatiles for meat spoilage. This study results in a concept for volatile based meat spoilage 
detection, but also shows the limitations of the hypothesis. In an artificial environment, volatiles can be emitted that are not important for the ecology of the blowfly, but highly informative for meat spoilage detection.

\subsection{References}

Aak,A., Knudsen,G.K. and Soleng,A. (2010) Wind tunnel behavioural response and field trapping of the blowfly Calliphora vicina. Medical and Veterinary Entomology, 24, 250-257.

Agafonova,L.E., Varushchenko,R.M., Druzhinina,A.I. and Polyakova,O.V. (2012) Phase equilibria and the thermodynamic properties of methyl and ethyl esters of carboxylic acids. 1. Methyl n-butanoate and ethyl propanoate. Journal of Chemical Thermodynamics, 47, 120-129.

Amendt,J., Krettek,R. and Zehner,R. (2004) Forensic entomology. Naturwissenschaften, 91, 51-65.

Arshadi,M. (2005) Emission of volatile organic compounds from softwood pellets during storage. Forest Products Journal, 55, 132-135.

Azeez,A.M., Meier,D., Odermatt,J. and Willner,T. (2010) Fast pyrolysis of African and European lignocellulosic biomasses using Py-GC/MS and fluidized bed reactor. Energy \& Fuels, 24, 2078-2085.

Balkovsky,E. and Shraiman,B.I. (2002) Olfactory search at high Reynolds number. Proceedings of the National Academy of Sciences of the United States of America, 99, 12589-12593.

Banerjee,S. (2001) Mechanisms of terpene release during sawdust and flake drying. Holzforschung, $55,413-416$.

Beck,J.J., Higbee,B.S., Light,D.M., Gee,W.S., Merrill,G.B. and Hayashi,J.M. (2012) Hull split and damaged almond volatiles attract male and female navel orangeworm moths. Journal of Agricultural and Food Chemistry. 60, 8090-8096.

Berijani,S., Assadi,Y., Anbia,M., Milani Hosseini,M.R. and Aghaee,E. (2006) Dispersive liquid-liquid microextraction combined with gas chromatography-flame photometric detection. Very simple, rapid and sensitive method for the determination of organophosphorus pesticides in water. Journal of Chromatography A, 1123, 1-9.

Blomquist,G.J. and Vogt,R.G. (2003) Insect pheromone biochemistry and molecular biology. Elsevier, San Francisco, USA.

Bohbot,J.D. and Dickens,J.C. (2012) Selectivity of odorant receptors in insects. Frontiers in Cellular Neuroscience, doi, 10.3389/fncel.2012.00029.

Bock,J., Kohl,D. and Schieberle,P. (1997) Discrimination between toasted and untoasted white bread. 11th European conference on solid-state transducers, EuroSensors XI, pp 27-30, Warsaw, Poland, ISBN 83-908335-0-6.

Branca,C., Di Blasi,C. and Elefante,R. (2006) Devolatilization of conventional pyrolysis oils generated from biomass and cellulose. Energy \& Fuels, 20, 2253-2261.

Branscheid,W., Honikel,K.O., v.Lengerken,G. and Troeger,K. (2007) Qualität von Fleisch- und Fleischwaren. Deutscher Fachverlag GmbH, Frankfurt am Main, Germany.

Briscoe,A.D. and Chittka,L. (2001) The evolution of colors vision in insects. Annual Review of Entomology, 46, 471-510. 
Burger,B.V., Munro,Z.M. and Visser,J.H. (1988) Determination of plant volatiles.1. Analysis of the insect-attracting allomone of the parasitic plant Hydnora-Africana using Grob-Habich activatedcharcoal traps. Journal of High Resolution Chromatography \& Chromatography Communications, 11, 496-499.

Castello,G., Moretti,P. and Vezzani,S. (2009) Retention models for programmed gas chromatography. Journal of Chromatography A, 1216, 1607-1623.

Chang,S.-T. (2001) Comparisons of the photostability of esterified wood. Polymer Degradation and Stability, 71, 261-266.

Chheda,J.N., Roman-Leshkov,Y. and Dumesic,J.A. (2006) Production of 5-hydroxymethylfurfural and furfural by dehydration of biomass-derived mono- and poly-saccharides. Green Chemistry, 9, 342350.

Choe,E. and Min,D.B. (2005) Chemistry and reactions of reactive oxygen species in foods. Journal of Food Science, 70, 142-159.

Combet,E., Henderson,J., Eastwood,C.D. and Burton,K.S. (2006) Eight-carbon volatiles in mushrooms and fungi, properties, analysis, and biosynthesis. Mycoscience, 47, 317-326.

Crnjar,R., Yin,C.M., Stoffolano,J.G., Barbarossa,I.T., Liscia,A. and Angioy,A.M. (1990) Influence of Age on the Electroantennogram Response of the Female Blowfly (Phormia-Regina) (Diptera, Calliphoridae). Journal of Insect Physiology, 36, 917-921.

Darwin,C. (1859) On the origin of species by means of natural selection, or the preservation of favoured races in the struggle for live. Oxford University Press, Oxford, United Kingdom.

Didomenico,J. and Rendhal,C.S. (2004) Remote emission sensing system and method with a composite beam of IR and UV radiation that is not split for detection. United States Patent, US 6,723,989, Apr. 20.

Dorea,H.S., Tadeo,J.L. and Sanchez-Brunete,C. (1996) Determination of organophosphorus pesticide residues in fruits by gas chromatography with ITD and NPD detection. Chromatographia, 43, 380-386.

Dufour,A., Girods,P., Masson,E., Normand,S., Rogaume,Y. and Zoulalian,A. (2007) Comparison of two methods of measuring wood pyrolysis tar. Journal of Chromatography A, 1164, 240-247.

Dutson,T.R. (1983) Relationship of $\mathrm{pH}$ and temperature to disruption of specific muscle proteins and activity of lysosomal proteases. Journal of Food Biochemistry, 7, 223-245.

Englund,F. and Nussbaum,R.M. (2000) Monoterpenes in scots pine and norway spruce and their emission during kiln drying. Holzforschung, 54, 449-456.

Ercolini,D., Russo,F., Nasi,A., Ferranti,P. and Villani,F. (2009) Mesophilic and psychrotrophic bacteria from meat and their spoilage potential in vitro and in beef. Applied and Environmental Microbiology, 75, 1990-2001.

Eßinger, T. M., Becker, B., Sauerwald, T., Kohl, C.-D., Paczkowski, S. and Schütz, S. (2010) Volatile gas sensors for large-scale drying of wood. Sensoren und Messsysteme - 15. ITG/GMA-Fachtagung. pp. 1-4, Nürnberg, Germany. 
Estevez,M., Morcuende,D., Ventanas,S. and Cava,R. (2003) Analysis of volatiles in meat from Iberian pigs and lean pigs after refrigeration and cooking by using SPME-GC-MS. Journal of Agricultural and Food Chemistry, 51, 3429-3435.

Evans,W.G. (2010) Reproductive role of infrared radiation sensors of Melanophila acuminata (Coleoptera, Buprestidae) at forest fires. Annals of the Entomological Society of America, 103, 823826.

Fackrell,J.E. and Robins,A.G. (1982) Concentration fluctuations and fluxes in plumes from point sources in a turbulent boundary layer. Journal of Fluid Mechanics, 117, 1-26.

Fengel,D. and Wegener,G. (1984) Wood, chemistry, ultrastructure, reactions. De Gruyter Inc., Boston, USA.

Feussner,I., Kühn,H. and Wasternack,C. (1997) Do specific linoleate 13-lipoxygenases initiate $\beta$ oxidation? FEBS Letters, 406, 1-5.

Fineschi,S. and Loreto,F. (2012) Leaf volatile isoprenoids, An important defensive armament in forest tree species. Iforest-Biogeoscience and Forestry, 5, 13-17.

Flematti,G.R., Scaffidi,A., Dixon,K.W., Smith,S.M. and Ghisalberti,E.L. (2011) Production of the seed germination stimulant karrikinolide from combustion of simple carbohydrates. Journal of Agricultural and Food Chemistry, 59, 1195-1198.

Forbes,S.L., Dent,B.B. and Stuart,B.H. (2005a) The effect of soil type on adipocere formation. Forensic Science International. 154, 35-43.

Forbes,S.L., Stuart,B.H. and Dent,B.B. (2002) The identification of adipocere in grave soils. Forensic Science International, 127, 225-230.

Forbes,S.L., Stuart,B.H. and Dent,B.B. (2005b) The effect of the burial environment on adipocere formation. Forensic Science International, 154, 24-34.

Forbes,S.L., Stuart,B.H. and Dent,B.B. (2005c) The effect of the method of burial on adipocere formation. Forensic Science International, 154, 44-52.

Frankel,E.N. (1983) Volatile lipid oxidation-products. Progress in Lipid Research, 22, 1-33.

Gao,M., Pan,D.X. and Sun,C.Y. (2003) Study on the thermal degradation of wood treated with amino resin and amino resin modified with phosphoric acid. Journal of Fire Science, 21, 189-201.

Giancoli,D.C. (2010) Physik, Lehr- und Übungsbuch. Pearson Education Deutschland GmbH, München, Germany.

Goldberg, M.E., Bhambhani, M. and Brandon,A. (1993) Aerosol hairspray containing low VOC content. United States Patent, No.: US 5,176,898.

Goll,D.E., Otsuka,Y., Nagainis,P.A., Shannon,J.D., Sathe,S.K. and Muguruma,M. (1983) Role of muscle proteinases in maintenance of muscle integrity and mass. Journal of Food Biochemistry, 7, 137-177.

Graedel,T. (1994) Industrial Ecology: Definition and Implementation. In: Industrial Ecology and Global Change, Eds. Socolow,R., Andrews,C., Berkhout,F., Thomas,V., Cambridge University Press, Cambridge, United Kingdom, p.23-43. 
Gramm,A. and Schütze,A. (2003) High performance solvent vapor identification with a two sensor array using temperature cycling and pattern classification. Sensors and Actuators B-Chemical, 95, 5865.

Granström,K. (2002) Emissions of monoterpenes and VOCs during drying of sawdust in a spouted bed. Forest Products Journal, 53, 48-55.

Grimaldi, D. and Engel, M. S. (2005) Evolution of the insects. Cambridge University Press, Cambridge, United Kingdom.

Grimmelikhuijzen,C.J.P. (2012) Mini-review, The evolution of neuropeptide signaling. Regulary Peptides, 177, 6-9.

Grob,K. and Zürcher,F. (1976) Stripping of trace organic substances from water, Equipment and procedure. Journal of Chromatography A, 117, 285-294.

Groot,M., Cokl,A. and Virant-Doberlet M. (2010) Effects of heterospecific and conspecific vibrational signal overlap and signal-to-noise ration on male responsiveness in Nezara viridula (L.). Journal of Experimental Biology, 213, 3213-3222.

Gruter,C., Schurch,R., Czaczkes,T.J., Taylor,K., Durance,T., Jones,S.M. and Ratnieks,F.L.W. (2012) Negative feedback enables fast and flexible collective decision-making in ants. PLoS ONE 7(9): e44501. doi:10.1371/journal.pone.0044501.

Gurr,M.I. (1988) Lipid-metabolism in man. Proceedings of the Nutrition Society, 47, 277-285.

Gutmacher,D., Hoefer,U. and Wöllenstein,J. (2011) Gas sensor technologies for fire detection. Sensors and Actuators B-Chemical, 175, 40-45.

Haglund,W.D. (1997) Dogs and coyotes, postmortem involvement with human remains. In: Haglund,W.D. and Sorg,M.H. (eds.), Forensic taphonomy, the postmortem fate of human remains. CRC Press, Boca Raton, pp.367-382.

Hallem,E.A. and Carlson,J.R. (2006) Coding of odors by a receptor repertoire. Cell, 125, 143-160.

Hansson,B.S. (1999) Insect Olfaction. Springer, New York.

Harris,W.E. and Habgood,H.W. (1966) Programmed temperature gas chromatography. John Wiley \& Sons, Inc., New York, USA.

Hayes,E.T., Leek,A.B.G., Curran,T.P., Dodd,V.A., Carton,O.T., Beattle,V.E. and O'Doherty,J.V. (2004) The influence of diet crude protein level on odour and ammonia emissions from finishing pig houses. Bioresource Technology, 91, 309-315.

Heath,R.J., Jackowski, S. and Rock,C.O. (2002) Fatty acid and phospholipid metabolism in prokaryotes. In: Vance,D.E. and Vance,J.E. (eds.), Biochemistry of lipids, lipoproteins and membranes. Elsevier, San Francisco, USA, pp.59-96.

Heil,M. and Karban,R. (2010) Explaining evolution of plant communication by airborne signals. Trends in Ecology and Evolution, 25, 137-144.

Heisswolf,A., Gabler,D., Obermaier,E. and Muller,C. (2007) Olfactory versus contact cues in host plant recognition of a monophagous chrysomelid beetle. Journal of Insect Behavior, 20, 247-266. 
Helmig,D. and Vierling,L. (1995) Water adsorption capacity of the solid adsorbents Tenax TA, Tenax GR, Carbotrap, Carbotrap C, Carbosieve SIII, and Carboxen 569 and water management techniques for the atmospheric sampling of volatile organic trace gases, Analytical Chemistry, 67, 4380-4386.

Higuchi,T. (1990) Lignin biochemistry, Biosynthesis and biodegradation. Wood Science and Technology, 24, 23-63.

Hopkins,D.W., Wiltshire,P.E.J. and Turner,B.D. (2000) Microbial characteristics of soils from graves, arm investigation at the interface of soil microbiology and forensic science. Applied Soil Ecology, 14, 283-288.

Hwang,C.J., Krasner,S.W., McGuire,M.J., Moylan,M.S. and Dale,M.S. (1984) Determination of subnanogram-per-liter levels of earthy-musty odorants in water by the salted closed-loop stripping method. Environmental Science and Technology, 18, 535-539.

Hübschmann,H.-J. (2001) Handbook of GC/MS. Wiley-VCH, Weinheim, Germany.

Ingram,L., Mohan,D., Bricka,M., Steele,P., Strobel,D., Crocker,D., Mitchell,B., Mohammad,J., Cantrell,K. and Pittman,C.U. (2008) Pyrolysis of wood and bark in an auger reactor, Physical properties and chemical analysis of the produced bio-oils. Energy \& Fuels, 22, 614-625.

Insausti,K., Goni,V., Petri,E., Gorraiz,C. and Beriain,M.J. (2005) Effect of weight at slaughter on the volatile compounds of cooked beef from Spanish cattle breeds. Meat Science, 70, 83-90.

Kai,M., Haustein,M., Molina,F., Petri,A., Scholz,B. and Piechulla,B. (2009) Bacterial volatiles and their action potential. Applied Microbiology and Biotechnology, 81, 1001-1012.

Kaib,M. (1974) Receptors for meat-odor and flower-odor on antenna of blowfly Calliphora vicina. Journal of Comparative Physiology, 95, 105-121.

Kalinova,B., Podskalska,H., Ruzicka,J. and Hoskovec,M. (2009) Irresistible bouquet of death-how are burying beetles (Coleoptera, Silphidae, Nicrophorus) attracted by carcasses. Naturwissenschaften, 96, 889-899.

Karagoz,S., Bhaskar,T., Muto,A. and Sakata,Y. (2005) Comparative studies of oil compositions produced from sawdust, rice husk, lignin and cellulose by hydrothermal treatment. Fuel, 84, 875-884.

Kim,S.K., Chang,H.W., Zellers,E.T. (2011) Microfabricated gas chromatograph for the selective determination of trichloroethylene vapor at sub-parts-per-billion concentrations in complex mixtures. Analytical Chemistry, 83, 7198-7206.

Kionka,C. and Kunau,W.H. (1985) Inducible B-oxidation pathway in Neurospora crassa. Journal of Bacteriology, 161, 153-157.

Kohl,C-D (1989) Surface processes in the detection of reducing gases with SnO2 based devices. Sensors and Actuators, 18, 71-113.

Kollmann,F. (1982) Technologie des Holzes und der Holzwerkstoffe, Anatomie und Pathologie, Chemie, Physik, Elastizität und Festigkeit. Springer, Berlin, Germany.

Kreiss,E., Schmitz,H. and Gebhardt,M. (2007) Electrophysiological characterisation of the infrared organ of the Australian "Little Ash Beetle" Acanthocnemus nigricans (Coleoptera, Acanthocnemidae). Journal of Comparative Physiology A-Neuroethology Sensory Neural and Behavioral Physiology, 193, 729-739. 
Kuske,M., Padilla,M., Romain,A.C., Nicolas,J., Rubio,R. and Marco,S. (2006) Detection of diverse mould species growing on building materials by gas sensor arrays and pattern recognition. Sensors and Actuators B-Chemical, 119, 33-40.

LeBlanc,H.N. and Logan,J.G. (2010) Exploiting insect olfaction in forensic entomology. In: Amendt,J., Campobasso,C.P., Goff,M.L. and Grassberger,M. (eds.), Current concepts in forensic entomology. Springer, New York, USA, pp.205-221.

Lee,J.H., Waller,J.C., Melton,S.L., Saxton,A.M. and Pordesimo,L.O. (2004) Feeding encapsulated ground full-fat soybeans to increase polyunsaturated fat concentrations and effects on flavor volatiles in fresh lamb. Journal of Animal Science, 82, 2734-2741.

Lewicki,M.S. (1998) A review of methods for spike sorting, the detection and classification of neural action potentials. Network: Computation in Neural Systems, 9, 53-78.

Linksvayer,T.A., Fewell,J.H., Gadau,J. and Laubichler,M.D. (2012) Developmental evolution in social insects, Regulatory networks from genes to society. Journal of Experimental Zoology, B-Molecular and Developmental Evolution, 318B, 159-169.

Madou,M.J. and Morrison,S.R. (1989) Chemical sensing with solid state devices. Elsevier, San Francisco, USA.

Mai,C., Kües,U. and Militz,H. Biotechnology in the wood industry. Applied Microbiology and Biotechnology, 63, 477-494.

Maida,R., Steinbrecht,A., Ziegelberger,G. and Pelosi,P. (1993) The pheromone binding protein of Bombyx mori, purification, characterization and immunocytochemical localization. Insect Biochemistry and Molecular Biology, 23, 243-253.

Maleknia,S.D., Bell,T.L. and Adams,M.A. (2009) Eucalypt smoke and wildfires, Temperature dependent emissions of biogenic volatile organic compounds. International Journal of Mass Spectrometry, 279, 126-133.

Manjare,S.D. and Ghoshal,A.K. (2006) Studies on adsorption of ethyl acetate vapor on activated carbon. Industrial \& Engineering Chemistry Research, 45, 6563-6569.

McNair,H.M. and Miller,M.M. (1997) Basic gas chromatography. Wiley VCH, New York, USA.

Merford,I. (2002) Review of the analytical techniques for sesquiterpenes and sesquiterpene lactones. Journal of Chromatography A, 967, 115-130.

Moreno-Opo,R., Margalida,A., Arredondo,A., Guil,F., Martin,M., Higuero,R., Soria,C. and Guzman,J. (2010) Factors influencing the presence of the cinereous vulture Aegypius monachus at carcasses, food preferences and implications for the management of supplementary feeding sites. Wildlife Biology, 16, 25-34.

Na,K. (2006) Determination of VOC source signature of vehicle exhaust in a traffic tunnel. Journal of Environmental Management, 81, 392-398.

Nation,J.L. (2001) Insect physiology and biochemistry. CRC Press, New York, USA.

Nawrath,T., Mgode,G.F., Weetjens,B., Kaufmann,S.H.E. and Schulz,S. (2012) The volatiles of pathogenic and nonpathogenic mycobacteria and related bacteria. Beilstein Journal of Organic Chemistry, 8, 290-299. 
Neuenschwander,U., Guignard,F. and Hermans,I. (2010) Mechanism of the aerobic oxidation of apinene. ChemSusChem, 3, 75-84.

Nordlund,D.A., Jones,R.L. and Lewis,W.J. (1981) Semiochemicals. Their role in pest control. Wiley $\mathrm{VCH}$, New York, USA.

Nufio,C.R. and Papaj,D.R. (2001) Host marking behavior in phytophagous insects and parasitoids. Entomologia Experimentalis et Applicata, 99, 273-293.

Nultsch,W. (2001) Allgemeine Botanik, Thieme Verlag, New York, USA.

Oasmaa,A., Kuoppala,E. and Solantausta,Y. (2003) Fast pyrolysis of forestry residue. 2. Physicochemical composition of product liquid. Energy \& Fuels, 17, 433-443.

Olafsdottir,G. and Kristbergsson,K. (2006) Electronic-nose technology, Application for quality evaluation in the fish industry. In: Nicolay,X. (ed.), Odors in the food industry. Springer, New York, USA, pp.57-74.

Osemwengie,L.I. and Steinberg,S. (2003) Closed-loop stripping analysis of synthetic musk compounds from fish tissues with measurement by gas chromatography-mass spectrometry with selected-ion monitoring. Journal of Chromatography A, 993, 1-15.

Oudia,A., Queiroz,J. and Simoes,R. (2008) Potential and limitation of Trametes versicolor laccase on biodegradation of Eucalyptus globulus and Pinus pinaster kraft pulp. Enzyme and Microbial Technology, 43, 144-148.

Paczkowski, S. and Schütz, S. (2011) Post-mortem volatiles of vertebrate tissue. Applied Microbiology and Biotechnology 91, 917-935.

Paczkowski, S., Sauerwald, T., Weiß.A., Bauer, M., Kohl, C.-D. and Schütz, S. (2011a) Biomimetic gas sensors for large-scale drying of wood particles. Martin-Palma, R. J. and Lakhtakia, A. 7975, 797505797505-8. Conference Proceedings of the SPIE: Bioinspiration, Biomimetics, and Bioreplication, San Diego, USA.

Paczkowski,S., Weißbecker,B., Schöning,M.J. and Schütz,S. (2011b) Biosensors on the basis of insect olfaction. In: Vilcinskas,A. (ed.), Insect Biotechnology. Springer, Dordrecht, The Netherlands, pp.225240.

Paczkowski, S., Paczkowska, M., Maibaum, F., and Schütz, S. (2012) Decaying mouse volatiles perceived by Calliphora vicina Rob.-Desv. Journal of Forensic Sciences. 57, 1497-1506.

Parkinson,P.R., Churchill,T.J., Rolls,W. (2008) Assessment of matrix effects on methyl benzoate, a potential biomarker for detection of outgassed semi-volatiles from mold in indoor building materials. Bulletin of Environmental Contamination and Toxicology. 81, 494-497.

Pelosi,P. and Maida,R. (1995) Odorant-binding proteins in insects. Comparative Biochemistry and Physiology B-Biochemistry \& Molecular Biology, 111, 503-514.

Peric-Mataruga,V., Lazarevic,J. and Nenadovic,V. (2001) A possible role for the dorsolateral protocerebral neurosecretory neurons in the trophic adaptations of Lymantria dispar (Lepidoptera, Lymantriidae). European Journal of Entomology, 98, 257-264.

Piskorz,J., Radlein,D. and Scott,D.S. (1986) On the mechanism of the rapid pyrolysis of cellulose. Journal of Analytical and Applied Pyrolysis, 9, 121-137. 
Plettner,E., Lazar,J., Prestwich,E.G. and Prestwich,G.D. (2000) Discrimination of pheromone enantiomers by two pheromone binding proteins from the gypsy moth Lymantria dispar. Biochemistry, 39, 8953-8962.

Pollien,P., Jordan,A., Lindinger,W. and Yeretzian,C. (2003) Liquid-air partitioning of volatile compounds in coffee, dynamic measurements using proton-transfer-reaction mass spectrometry. International Journal of Mass Spectrometry, 228, 69-80.

Poole,C. (2012) Gas chromatography. Elsevier, San Francisco, USA.

Rajamaki,T., Alakomi,H.L., Ritvanen,T., Skytta,E.S., Smolander,M. and Ahvenainen,R. (2006) Application of an electronic nose for quality assessment of modified atmosphere packaged poultry meat. Food Control, 17, 5-13.

Ravaglioli,A., Krajewski,A., Celotti,G.C., Piancastelli,A., Bacchini,B., Montanari,L., Zama,G. and Piombi,L. (1996) Mineral evolution of bone. Biomaterials, 17, 617-622.

Reyes-Vidal,Y. and de la Torre,M. (2009) Emission, perception, and behavioral responses of entomopathogenic nematodes to semiochemicals. Nematropica, 39, 213-223.

Riolo,P., Minuz,R.L., Anfora,G., Stacconi,M.V.R., Carlin,S., Isidoro,N. and Romani,R. (2012) Perception of host plant volatiles in Hyalesthes obsoletus, Behavior, morphology, and electrophysiology. Journal of Chemical Ecology, 38, 1017-1030.

Risholm-Sudman,M., Lundgren,M., Vestin,E. and Herder,P. (1998) Emissions of acetic acid and other volatile organic compounds from different species of solid wood. Holz als Roh- und Werkstoff, 56, 125-129.

Roffael,E. (2006) Volatile organic compounds and formaldehyde in nature, wood and wood based panels. Holz als Roh- und Werkstoff, 64, 144-149.

Rosa Ras,M., Borrull,F. and Maria Marce,R. (2009) Sampling and preconcentration techniques for determination of volatile organic compounds in air samples. Trends in Analytical Chemistry, 28, 347361.

Rothweiler,H., Waeger,P.A. and Schlatter,C. (1991) Comparison of Tenax TA and Carbotrap for sampling and analysis of volatile organic compounds in air. Atmospheric Environment, Part B, 25B, 231-235.

Ruther,J., Meiners,T. and Steidle,J.L.M. (2002) Rich in phenomena-lacking in terms. A classification of kairomones. Chemoecology, 12, 161-167.

Sakurai,T., Nakagawa,T., Mitsuno,H., Mori,H., Endo,Y., Tanoue,S., Yasukochi,Y., Touhara,K. and Nishioka,T. (2004) Identification and functional characterization of a sex pheromone receptor in the silkmoth Bombyx mori. Proceedings of the National Academy of Sciences of the United States of America, 101, 16653-16658.

Satelle, D.B., Hall, L.M. \& Hildebrand J.G. (1979) Receptors for neurotransmitters, hormones and pheromones in insects. Biomedical Press, Elsevier North-Holland, The Netherlands.

Sauer,A.E., Karg,G., Koch,U.T., Kramer,J.J.D. and Milli,R. (1992). A portable EAG system for the measurement of pheromone concentrations in the field. Chemical Senses, 17, 543-553. 
Sauerwald,T., Skiera,D. and Kohl,C.D. (2007) Selectivity enhancement of gas sensors using nonequilibrium polarisation effects in metal oxide films. Applied Physics A-Materials Science \& Processing, 87, 525-529.

Schaefer,M. (2012) Wörterbuch der Ökologie. Spektrum Verlag, Heidelberg, Germany.

Schmidt,R.F., Lang,F. and Heckmann,M. (2011) Physiologie des Menschen. Springer, Berlin, Germany.

Schmitz,H., Schmitz,A., Trenner,S. and Bleckmann,H. (2002) A new type of insect infrared organ of low thermal mass. Naturwissenschaften, 89, 226-229.

Schneider, D. (1957). Elektrophysiologische Untersuchungen von Chemo- und Mechanorezeptoren der Antenne des Seidenspinners Bombyx mori L. Journal of Comparative Physiology A, 40, 8-41.

Schöning,M.J., Schütz,S., Schroth,P., Weissbecker,B., Steffen,A., Kordos,P., Hummel,H.E. and Luth,H. (1998) A BioFET on the basis of intact insect antennae. Sensors and Actuators B-Chemical, 47, 235-238.

Schütz,S., Weißbecker,B., Hummel,H.E., Schöning,M.J., Riemer,A., Kordos,P. and Lüth,H. (1997a) Field effect transistor-insect antenna junction. Naturwissenschaften. 84, 86-88.

Schütz,S., Weißbecker,B., Klein,A. and Hummel,H.E. (1997b) Host plant selection of the Colorado potato beetle as influenced by damage induced volatiles of the potato plant. Naturwissenschaften. 84, 212-217.

Schütz,S., Weißbecker,B., Hummel,H.E., Apel,K.-H., Schmitz,H. and Bleckmann,H. (1999a) Insect antenna as a smoke detector. Nature. 398, 298-299.

Schütz,S., Weißbecker,B., Koch,U.T. and Hummel,H.E, (1999b). Detection of volatiles released by diseased potato tubers using a biosensor on the basis of intact insect antennae. Biosensors and Bioelectronics, 14, 221-228.

Schütz,S. and Weißbecker,B. (2003) Mechanismen duftvermittelter Pflanze-Insekt-Interaktionen, Vom Pflanzenstreß zum Pflanzenfraß. Nova Acta Leopoldina. 87, 215-235.

Schütz,S., Weißbecker,B., Apel,K.-H. and Wenk,M. (2004) Duftstoffsignale als Marker für die Befallsdisposition von Kiefern durch den Blauen Kiefernprachtkäfer Phaenops cyanea F. (Col., Buprestidae). Mitteilungen der Deutschen Gesellschaft für allgemeine und angewandte Entomologie, 14, 301-306.

Semancik,S., Cavicchi,R.E., Wheeler,M.C., Tiffany,J.E., Poirier,G.E., Walton,R.M., Suehle,J.S., Panchapakesan,B. and DeVoe,D.L. (2001) Microhotplate platforms for chemical sensor research. Sensors and Actuators, B-Chemical, 77, 579-591.

Shanbhag,S., Muller,B. and Steinbrecht,A. (1999) Types, external organization, innervation and distribution of olfactory sensilla. International Journal of Insect Morphology and Embryology, 28, 377397.

Shepherd,W.P., Huber,D.P.W., Seybold,S.J. and Fettig,C.J. (2007) Antennal responses of the western pine beetle, Dendroctonus brevicomis (Coleoptera : Curculionidae), to stem volatiles of its primary host, Pinus ponderosa, and nine sympatric nonhost angiosperms and conifers. Chemoecology, 17, 209-221. 
Singh,R.N. (1998) Neurobiology of the gustatory system of Drosophila and some terrestrial insects. Microscopy Research and Technique, 39, 547-563.

Sink,J.D. (1979a) Factors influencing the flavour of muscle foods. Journal of Food Science, 44, 1-5.

Sink,J.D. (1979b) Symposium on meat flavor factors influencing flavor of muscle foods. Journal of Food Science, 44, 1-5.

Sjostrom,E. (1993) Wood Chemistry, Second Edition, Elsevier, San Francisco, USA.

Smid,H.M., van Loon,J.J.A., Posthumus,M.A. and Vet, L.E.M. (2002) GC-EAG-analysis of volatiles from Brussels sprouts plants damaged by two species of Pieris caterpillars, olfactory receptive range of a specialist and a generalist parasitoid wasp species. Chemoecology, 12, 169-176.

Smith,K.G.V. (1986) A manual of forensic entomology. Department of Entomology, British Museum (Natural History), London, United Kingdom.

Sos,J., Kemeny,T., Rigo,J. and Budavari,I. (1961) Influence of amino acid deficiency on chemical constitution and solidity of bones. Acta Physiologica Academiae Scientiarum Hungaricae, 19, 267-272.

Stanford,M.A. and Mcgorrin,R.J. (1994) Flavor volatilization in microwave food model systems. Thermally Generated Flavors, 543, 414-436.

Statheropoulos,M., Spiliopoulou,C. and Agapiou,A. (2005) A study of volatile organic compounds evolved from the decaying human body. Forensic Science International, 153, 147-155.

Steffen,A. and Pawliszyn,J. (1996) Analysis of flavor volatiles using headspace solid-phase microextraction. Journal of Agriculture and Food Chemistry, 44, 2187-2193.

Storch,V., Welsch,U. and Wink,M. (2001) Evolutionsbiologie. Springer, New York, USA.

Svedberg,U.R.A., Högberg,H.E., Högberg,J. and Galle,B. (2004) Emission of hexanal and carbon monoxide from storage of wood pellets, a potential occupational and domestic health hazard. Annals of Occupational Hygiene, 48, 339-349.

Tegoni,M., Campanacci,V. and Cambillau,C. (2004) Structural aspects of sexual attraction and chemical communlication in insects. Trends in Biochemical Sciences, 29, 257-264.

Thakeow,P., Angeli,S., Weissbecker,B. and Schütz,S. (2008) Antennal and behavioral responses of Cis boleti to fungal odor of Trametes gibbosa. Chemical Senses, 33, 379-387.

The good scents company (06.2012) http,

//www.thegoodscentscompany.com/search.php?qName=\&submit.x=3\&submit.y=6,

Tholl,D., Boland,W., Hansel,A., Loreto,F., Rose,U.S.R. and Schnitzler,J.P. (2006) Practical approaches to plant volatile analysis. Plant Journal, 45, 540-560.

Tjeerdsma,B.F., Boonstra,M., Pizzi,A., Tekely,P. and Militz,H. (1998) Characterisation of thermally modified wood, molecular reasons for wood performance improvement. Holz als Roh- und Werkstoff, 56, 149-153.

Vandermoten,S., Mescher,M.C., Francis,F., Haubruge,E. and Verheggen,F.J. (2012) Aphid alarm pheromone: An overview of current knowledge on biosynthesis and functions. Insect Biochemistry and Molecular Biology, 42, 155-163. 
Van der Pers,J.,N.,C. and Minks,A.K. (2011) Pheromone monitoring in the field using single sensillum recording. Entomologia Experimentalis et Applicata, 68, 237-245.

Van Hamme,J.D. and Ward,O.P. (2000) Development of a method for the application of solid-phase microextraction to monitor biodegradation of volatile hydrocarbons during bacterial growth on crude oil. 25, 155-162.

Vass,A.A., Smith,R.R., Thompson,C.V., Burnett,M.N., Wolf,D.A., Synstelien,J.A., Dulgerian,N. and Eckenrode,B.A. (2004) Decompositional odor analysis database. Journal of Forensic Sciences, 49, 760-769.

Vass,A.A., Smith,R.R., Thompson,C.V., Burnett,M.N., Dulgerian,N. and Eckenrode,B.A. (2008) Odor analysis of decomposing buried human remains. Journal of Forensic Sciences, 53, 384-391.

Vass,A.A., Barshick,S.A., Sega,G., Caton,J., Skeen,J.T., Love,J.C. and Synstelien,J.A. (2002) Decomposition chemistry of human remains, A new methodology for determining the postmortem interval. Journal of Forensic Sciences, 47, 542-553.

Verheggen,F.J., Arnaud,L., Bartram,S., Gohy,M. and Haubruge,E. (2008) Aphid and plant volatiles induce oviposition in an aphidophagous hoverfly. Journal of Chemical Ecology. 34, 301-307.

Villermaux,E. and Innocenti,C. (1999) On the geometry of turbulent mixing. Journal of Fluid Mechanics, 393, 123-148.

Vogt,R.G. and Riddiford,L.M. (1981) Pheromone binding and inactivation by moth antennae. Nature, 293, 161-163.

Wardencki,W., Michulec,M. and Curylo,J. (2004) A review of theoretical and practical aspects of solid phase microextraction in food analysis. International Journal of Food Science \& Technology, 39, 703-717.

Waser,R. (2003) Nanoelectronics and information technology - Advanced electronic materials and novel devices. Wiley- $\mathrm{VCH}$, Berlin, Germany.

Watson,P.R., Van Hove,M.A. and K. Hermann,K. (2004) NIST surface structure database - Version 5.0 national institute of standards and technology. Gaithersburg, Maryland, USA.

Weissbecker,B., Van Loon,J.J.A., Posthumus,M.A., Bouwmeester,H.J. and Dicke,M. (2000) Identification of volatile potato sesquiterpenoids and their olfactory detection by the two-spotted stinkbug Perillus bioculatus. Journal of Chemical Ecology, 26, 1433-1445.

Weissbecker,B., Holighaus,G. and Schütz,S. (2004) Gas chromatography with mass spectrometric and electroantennographic detection, analysis of wood odorants by direct coupling of insect olfaction and mass spectrometry. Journal of Chromatography A, 1056, 209-216.

Wicher,D., Schafer,R., Bauernfeind,R., Stensmyr,M.C., Heller,R., Heinemann,S.H. and Hansson,B.S. (2008) Drosophila odorant receptors are both ligand-gated and cyclic-nucleotide-activated cation channels. Nature, 452, 1007-1010.

Winquist,F., Hornsten,E.G., Sundgren,H. and Lundstrom,I. (1993) Performance of an electronic nose for quality estimation of ground meat. Measuring Science and Technology. 4, 1493-1500.

Wolska,L., Gdaniec-Pietryka,M., Konieczka,P. and Namiesnik,J. (2008) Problems of PAH quantification by GC-MS method using isotope-labelled standards. Talanta, 78, 730-735. 
Yaws,C.L. (2007) The Yaws handbook of vapor pressure, antoine coefficients. Gulf Publishing Company, Houston, Texas, USA.

Zeng,T.X., Zhao,B.J. and Zhu,S.F. (2010) Thermodynamic analysis of the sublimation process in cadmium selenide. Journal of Alloys and Compounds, 491, 170-172.

Zhang,B., Huang,H.J. and Ramaswamy,S. (2008) Reaction kinetics of the hydrothermal treatment of lignin. Applied Biochemistry and Biotechnology, 147, 119-131 


\section{Biosensors on the basis of insect olfaction (Book chapter)}

Authors: Paczkowski, S., Weissbecker, B., Schöning, M.J., Schütz, S.

Published in: Vilcinskas, A. (ed.) (2011) Insect Biotechnology, Biologically-Inspired Systems, Volume 2, p. 225-240. 


\title{
Chapter 12 \\ Biosensors on the Basis of Insect Olfaction
}

\author{
Sebastian Paczkowski, Bernhard Weißbecker, Michael J. Schöning, \\ and Stefan Schütz
}

\begin{abstract}
Biosensors utilize organic components like proteins, cells, or fragments of organisms within a technical device. By combining technical transducers with a biological sensory unit it is possible to reproduce the capability of natural sensors, which far exceed mere technical solutions in selectivity and sensitivity. For instance, by integrating the antenna of a Colorado potato beetle (Leptinotarsa decemlineata) in the gate of a field-effect transistor (BioFET), volatile trace compounds are directly detectable in the ppb range. A complex biochemical detection system in the antenna of the beetle serves as a filter for compounds that provide vital information about its environment. Another advantage of biologically inspired sensory solutions is the evolutionary tuning of the natural detection units towards compounds that are significantly correlated to ecological conditions. Nature has undergone millions of years of survival of the fittest to select compounds that serve organisms reliably as information to trace hosts, mating partners, enemies, or competitors. Because of the diversity of ecological interactions between animals all over the world the biosensor concept and biomimetic approaches can lead to numerous applications for tracking, surveillance and monitoring of environmental and industrial processes.
\end{abstract}

Keywords Volatile organic compounds (VOCs) - BioFET · Fire detection · Agricultural pests · Forest pests - Post mortem interval estimation · Meat spoilage detection

\subsection{Definition and Basic Principles}

Basically, a biosensor is a measuring device that traces chemical compounds, organisms, or physical measurands by spatially and functionally combining a biological

\author{
S. Paczkowski (B) \\ Department of Forest Zoology and Forest Conservation, Buesgen-Institute, \\ Georg-August-University Göttingen, 37077 Göttingen, Germany \\ e-mail: spaczko@gwdg.de
}


component with a physical or chemical transducer (Lowe, 1985; Scheller et al., 1985). This definition suggests a wide field of possible applications, as it neither refers to any peculiar biological components nor does it describe any concrete physical measurands. Actually, the field of possible realizations of biosensor concepts is quite extensive.

The integrated biological component could, for instance, be an organic molecule, Like an enzyme or an antibody. These biochemical structures perform distinctive tasks in organisms and therefore, interact with only a selected number of other chemical compounds that occur sometimes only in low concentrations. This selectivity can be utilized in a sensor concept that responds selectively and sensitively towards distinct compounds.

The bio-component can also be an organelle, cell, organ, or a complete organism. Here, the definition of a biosensor converges towards the definition of a bio-indicator that is defined as a plant or animal species whose appearance or absence in a habitat allows correlations to environmental conditions, like nutrition availability or air pollution. As in the case of a bio-indicator where a macroscopic measurand, for instance the number of lichen-species, is determined, also a biosensor on the basis of molecular bio-components has to display a measurable factor that is transduced into a physical measurand.

This leads to the second element of a biosensor: the so-called transducer. As the technological part of the sensor this compound is defined by its function and not by its mode of operation. Its task is to convert the specific biological/chemical interaction of the bio-component and an analyte into a physical/chemical response with a measurable output, generally electrical signals.

The first published application of a biosensor was a system to measure the concentration of glucose in the blood (Clark and Lyons, 1962). Here, the enzyme glucose oxidase was immobilized with a dialysis membrane on a platinum electrode. The enzyme metabolizes glucose to gluconolactone while the produced hydrogen is transformed to hydrogen peroxide under consumption of oxygen. This product is detected by an amperometric electrode. Alternatively, the consumption of oxygen can be recorded. The bio-component is in this case the dissolved glucose oxidase and the transducer, a platinum oxygen electrode. A permeable membrane keeps the solution around the electrode.

\subsection{Types of Biosensors}

Biosensors can be classified by the type of the used bio-component, the stage of development (generation), or the type of transducer.

\subsubsection{Bio-Components}

Enzymes (enzyme sensors) and antibodies (immuno sensors) are utilized as biocomponents in biosensors where the substrate is detected by docking on a selective 
receptor. One can differentiate between catalytic sensors and affinity sensors depending on whether the formation of a receptor/substrate complex yields a metabolic product or not.

A microbial sensor utilizes living cells, for instance coupled to an oxygen electrode that measures respiration processes of the cell by means of the oxygen uptake. This utilization of living cells or tissue opens new perspectives for biosensor applications. However, it also presents a technical challenge to cultivate functional cells on a transducer and to obtain a sufficient storage protocol for the biosensor (Rudolph and Reasor, 2001). Biological receptors or organisms (e.g., after genetic modification) can even be used for the detection of compounds that are not relevant to the organism itself but are of greater interest to humans. As an example, genetically engineered bacteria produce a fluorescent protein in the presence of uranium, which emits a green glow when irradiated by UV-light (Hillson et al., 2007). Another example is the application of complete organs, for instance insect antennae, which is described in detail in Section 5.

\subsubsection{Generations of Biosensors}

The above-mentioned glucose sensor of Clark and Lyons uses a membrane in order to immobilize enzymes on an electrode. This setup is also designated as a biosensor of the first generation (Scheller et al., 1985).

Enhancements to this technique are sensors where the biochemical receptors are directly bound to the surface of the transducer (2nd generation) or immobilized directly on an electronic control device, for instance a transistor (3rd generation). In particular, field-effect transistors (FETs) are convenient because of their high input impedance. The combination of a FET with a bio-component, for instance an insect antenna, is referred to as a BioFET (Schügerl, 1985; Schütz et al., 1997a).

\subsubsection{Transducers}

The most important types of transducers are electrochemical, optical, masssensitive, or thermal sensors (Rodriguez-Mozaz et al., 2006). An example of electrochemical transduction is the amperometric detection mentioned in Section 1, which is especially used for catalytic enzyme reactions.

Optical sensors can utilize the absorption, fluorescence, or bioluminescence of molecules and organisms as input.

One technical realization of mass-sensitive sensors is the piezoelectric sensor combining the surface of a quartz oscillator with an enzyme or antibody. When biocomponents from the surroundings attach to its surface the total mass of the crystal changes and due to Sauerbrey's equation the oscillating frequency of the quartz also changes.

Thermal sensors detect the production or consumption of heat during biochemical reactions. 


\subsection{Applications of Biosensors}

Conventional methods of instrumental chemical analysis, especially gas chromatography and mass spectrometry (GC-MS), require high laboratory costs. In this context, biosensors offer approaches for improved methods that allow real-time on-site analytics (Badihi-Mossberg et al. 2007).

To detect, for instance, a pesticide in farmland, standard methods require a labor-intensive extraction of the target compounds from the sample matrix. GCMS analysis is then performed with a throughput of 20-40 samples maximum per day. Therefore, the manual labor for sample preparation and the operation of the instrumental setup is very cost-intensive, restricting these conventional methods to the survey of a limited number of samples.

A portable measuring device without extensive sample preparation is needed to establish convenient applications for practical on-site use (Rodriguez-Mozaz et al., 2006). A biosensor with an appropriate selectivity can detect compounds directly in their matrix without preceding extraction or purification. This will inter alia enable the recording of data in a high spatial and temporal resolution in order to follow a concentration gradient and thereby, localize the source of the pollution. Applications of biosensors are, for instance, the detection of pesticides, air pollutants such as formaldehyde or sulfur dioxide, polycyclic aromatic hydrocarbons (PAHs), herbicides, toxicants, or heavy metals (Badihi-Mossberg et al., 2007). The range of applications for biosensors is not restricted to the detection of chemical compounds, but also includes the sensitive and highly reliable detection of specific microorganisms (Turner et al., 2008). Examples are the detection of Escherichia coli in the meat-processing industry (Tokarskyy and Marshall, 2008) or in water (Theegala et al., 2008). However, biosensors provide less accurate quantifications of compounds in comparison with conventional techniques of trace analysis.

If there is an assessment of complex biological effects needed, like toxicity and mutagenicity of mixtures, the state of health of plants and animals as well as the quality of ecosystems, a biological integration of complex measurands is necessary which cannot be detected by compound-specific trace analysis. In this case, a technical combination of bio-indicator organisms to adapted transductors is necessary. An example is the detection of the biochemical oxygen demand (BOD) of water which displays the amount of oxygen demanded to catabolize the contained amount of organic matter. This complex measurand is an indicator for the rate of pollution by sewage and can be determined with different conventional methods that are not applicable for online measurement (Rodriguez-Mozaz et al., 2006). For this demanding task biosensor approaches are already commercially available (Liu and Mattiasson, 2002).

On the whole, it is to be expected that the role of biosensors in diverse fields of application will increase rapidly in the future. Some applications are already covered by commercially available systems and recent research highlights the large diversity of further possibilities. 


\subsection{Insect Olfaction as a Basis for Biosensors}

The basic idea of a biosensor based on insect olfaction is to utilize the extremely high sensitivity and selectivity of olfactory receptors (ORs). The silk moth Bombyx mori is able to trace 1,000 molecules per second of the pheromone bombycol in one cubic centimeter of air (Kaissling and Priesner, 1970). This is equivalent to a 1-g sugar lump diluted within and distributed over the whole water volume of Lake Constance. Another advantage of an insect antenna is the evolutionary adaption of insect olfaction to the detection of complex environmental parameters. The black jewel beetle Melanophila acuminata can, for instance, detect the burnt scent of a single charred tree over a distance of several kilometers (Schütz et al., 1999).

Therefore, the combination of a highly specialized biochemically operating insect organ with a signal amplifying and processing electronic device can lead to a striking detection performance of organic trace compounds in the atmosphere. Such a biosensor system acquires its sensitivity and selectivity by means of a biochemical transduction pathway linking the occurrence of organic trace compounds to the depolarization of olfactory sensor neuron populations on the antenna. As the adaptation of the olfactory sense in insects to the distinctive demands for the single species has gone through millions of years of selection of the fittest, the resulting compounds can be regarded as marker compounds in the ecological interaction between the insect and its environment. Combining techniques of trace analysis, like gas chromatography/mass spectrometry with parallel electroantennographic detection based on insect antennae into one GC-MS/EAD setup (Weißbecker et al., 2004) allows the identification of single compounds that are selectively traceable by an insect via scent. As insect species appear in most ecosystems all over the world and are adapted to many, often very distinct olfaction-based interactions with their environment, the amount of potentially usable marker compounds for biosensors based on insect olfaction is extremely large. The applicability of these marker compounds for economic purposes implies that the outcome of biodiversity conservation is a considerable source of innovation.

\subsubsection{The Biochemical Transduction Pathway in Insect Olfaction}

Once an odor plume reaches the airspace around an insect, it enters nanopores in the cuticle of an olfactory sensillum. The cuticle covers the antenna in order to prevent mechanical damage and desiccation of the sensory neurons housed in the sensillum. These nanopores allow volatile organic compounds to diffuse into the sensillum lymph that surrounds the neurons inside the sensillum. So-called odorant binding proteins (OBPs), first discovered by Vogt and Riddiford as pheromone-binding proteins (PBPs) (Vogt and Riddiford, 1981), selectively bind and transport the mostly 
Fig. 12.1 The functional concept of insect olfaction. Embedded on the surface of the neuronal membrane inside a sensillum on the antenna are receptors that trigger ion influx by contact with an OBP-ligand complex

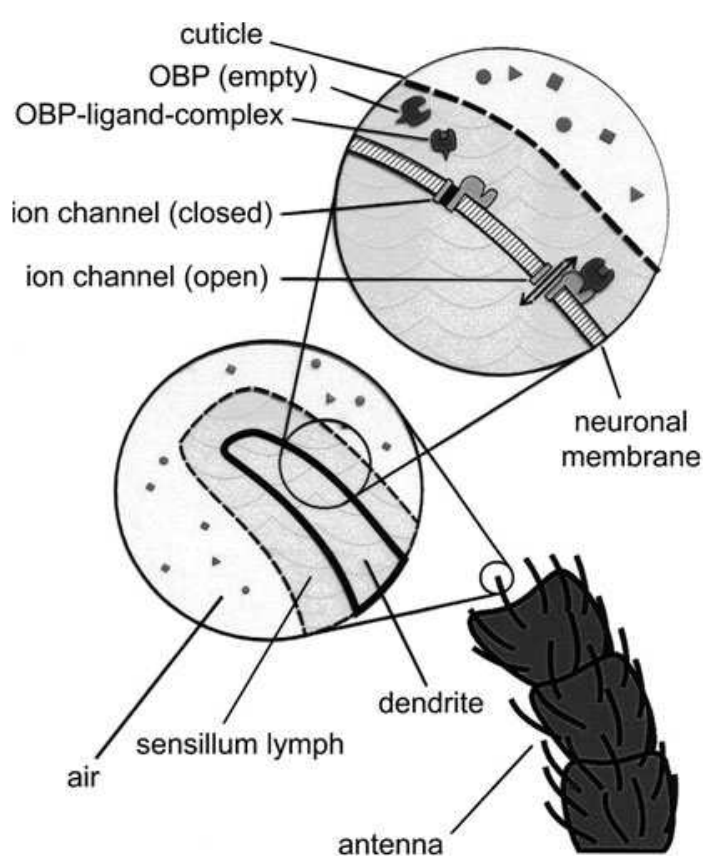

hydrophobic odor compounds through the hydrophilic sensillum lymph to the olfactory receptor contained in the neuron membrane (Fig. 12.1). It is proposed that due to the three-dimensional tertiary structure of these mono-chain proteins a binding cavity is formed in which functional groups of amino acids are exposed to interact with the peculiar structure and charge distribution of the odor molecule (Tegoni et al., 2004). When the OBP-ligand complex contacts its specific olfactory receptors on the neuron surface, a G-coupled protein cascade can be activated which leads to the opening of an ion channel allowing an influx of potassium ions that depolarizes the sensillum neuron. When the odor is present in a detectable concentration a sufficient number of cascades are activated to exceed the neuronal activation threshold and an action potential is elicited and further processed in the insect nervous system. Although intensive research has been conducted on understanding the biochemical interactions involved in insect olfaction the interactions are not as yet fully understood. For instance, the role of the OBPs and the ORs regarding the priority of selectiveness in binding the odor molecule is still a matter of discussion (Rützler and Zwiebel, 2005) and, as a second example, recently it has been found that the $\mathrm{OR}$ and the ion channel can form a spatial functional unit omitting the G-coupled protein transduction pathway (Wicher et al., 2008). However, the striking performance of insect olfaction in distinctively corresponding to ecologically relevant compounds has been verified by many studies and should lead to a wide range of artificial olfaction-based sensor applications in the future. 


\subsection{Application Layout: Biosensors on the Basis of Insect Antennae}

The utilization of an intact insect antenna as a bio-component requires a mechanically and electrically stable junction while avoiding damage to the organ. Because of advantageous electrical and constructive possibilities the design of biosensors as BioFETs, meaning a hybrid device consisting of an insect antenna and a field-effect transistor (Fig. 12.2), has been established as particularly reliable (Schöning et al., 1998; Schroth et al., 2001).

In the case of the "whole-beetle" setup, the antenna of the Colorado potato beetle was directly coupled to the gate of the field-effect transistor via an electrolyte solution, whereas for the "isolated antenna" setup, it was removed from the beetle (here, one part of the antenna is connected via the electrolyte to the FET gate). A typical dose-response curve of this antenna-based BioFET is schematically depicted in Fig. 12.3: An air current, loaded with (Z)-3-hexen-1-ol, as an exemplary odor compound, is applied to the antenna. The biochemical cascade inside the antenna finally leads to the formation of an electrical potential drop across the receptor's cell membrane and thus, to a dipole potential over the whole antenna. The latter modulates the conductance of the FET channel between source and drain, inducing a distinct variation of the drain current $I_{\mathrm{D}}$, which is dependent on the particular odor concentration.

The immanent miniaturization of the insect antenna as well as the miniaturization of the microelectronic part will allow manufacture of biosensor circuits of the size of a $1 €$ coin. Such sensor heads are deployable in portable biosensor systems to enable high-resolution data acquisition in-situ.

As distinctive examples four potential applications of biosensors on the basis of insect antennae are described below.

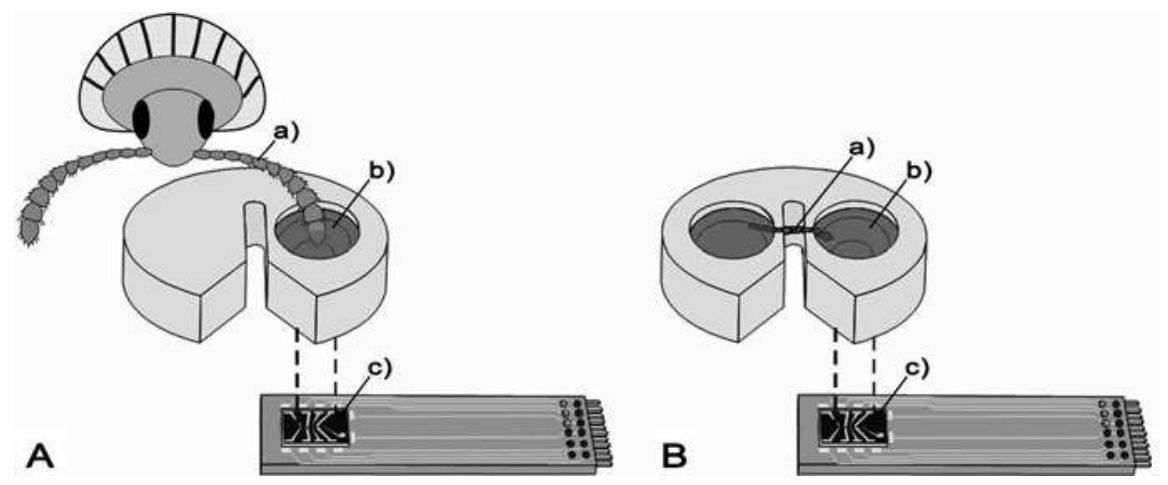

Fig. 12.2 BioFET hybrid consisting of an insect antenna and a field-effect transistor (FET) with intact Colorado potato beetle (A) and isolated antenna setup (B), respectively. (a) Antenna, (b) electrolyte, and (c) field-effect transistor 
Fig. 12.3 Sensor response of the BioFET towards different concentrations of (Z)-3-hexen-1-ol in air. The change in the drain current $I_{\mathrm{D}}$ serves as a sensor signal

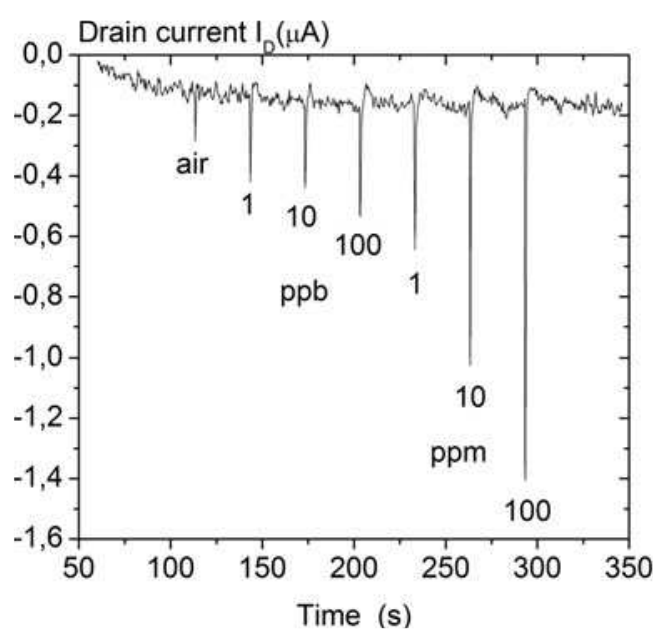

\subsubsection{Fire Detection with Insect Antennae}

Already at the beginning of the twentieth century there were reports from firemen of black beetles that flew in masses towards burning forests, hampering efforts to extinguish fires and even attacking firemen. Reports on this unusual pyrophilic behavior of the black jewel beetle $M$. acuminata included speculation that the beetle might be able to orientate towards a forest fire through olfactory means.

Further research on this beetle revealed that its antenna detects smoke components such as guajacol compounds very sensitively (Fig. 12.4).

These compounds originate from the pyrolysis of lignin, one of the basic constituents of wood. As the beetles breed in burned wood, they are adapted to the detection of wood fires like forest fires. Combustion of fossil wood (i.e., coal) can be detected by the beetles, too, as it also contains lignin.

Fig. 12.4 Result of combined gas chromatography/flame ionization detection (GC-FID/EAD) utilizing the excised antenna fragment of Melanophila acuminata. Simultaneous peak formation allows the identification of electrophysiologically active compounds, in this case guajacol derivates and terpenes

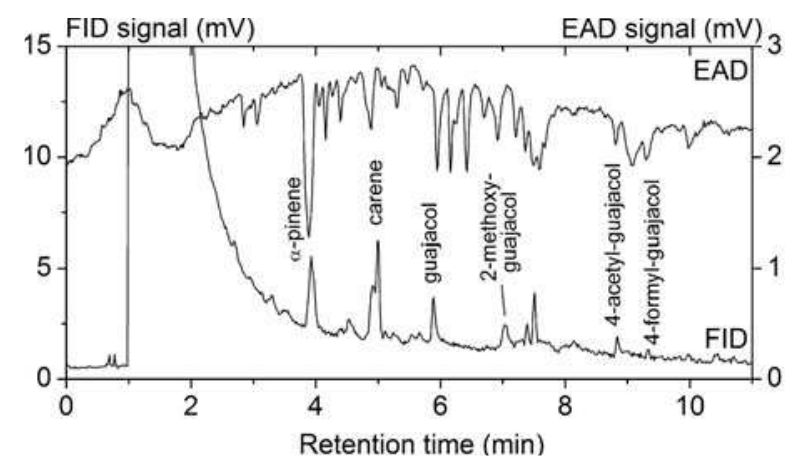


Combustion products of diesel fuel or gasoline do not elicit comparable signals. This enables the design of a biosensor based on the antenna of M. acuminata to differentiate between frequently occurring background compounds, for example originating from traffic, and wood fire. Thus, false-alarms caused by engine exhausts can be avoided and a reliable fire early warning system can be based on the performance of the biosensor. Additionally, by distinguishing between different guajacol compounds the beetle is able to differentiate between types of fires, as the compounds originate from the variation of oxygen availability and fire temperature. As a fire warning device should be integrated into an emergency plan any additional information about the kind of fire will facilitate selection of appropriate response procedures.

Field experiments revealed that a fire comprising of only a few trees $\left(5 \mathrm{~m}^{3}\right.$ wood) can be detected by a biosensor based on the antenna of $M$. acuminata over a $2-\mathrm{km}$ distance and can be traced by the concentration gradient of smoke components and wind direction through a dense forest stand. Combining the high sensitivity and selectivity for marker compounds of fires with the identification of the kind of fire and the traceability of the source, a biosensor based on the antenna of $M$. acuminata is a powerful tool to be used as the first link in the fire-fighting chain.

\subsubsection{Detection of Phytophagous Infestation in Agricultural Crops}

The Colorado potato beetle Leptinotarsa decemlineata is one of the most important agricultural pests. Originally, the beetle occurred only on Solanum rostratum which is a solanaceous herb like the potato plant Solanum tuberosum. When American settlers intensified cultivation of potatoes the beetle infested these cultures as an additional source of nutrition. Since then the Colorado potato beetle has spread over the whole world and caused serious crop losses.

Investigations into the olfactory capabilities of $L$. decemlineata in search of a possible pest-management technique revealed that the beetle is able to differentiate between the feeding of conspecifics, infection of potato plants with the late blight disease (Phytophthora infestans), as well as the mechanical damage of potato plants via hail and agricultural machines by its olfaction. Thereby, it uses the fact that potato plants emit different scents when beetles feed on them compared to plants that are infected by fungi or that are mechanically damaged (Schütz et al. 1997b) and it uses different marker compounds for this discrimination.

These marker compounds are detected in very low concentrations and therefore, are recognized by the beetle even at long distances from the emission source. A marker compound for the cumulative type of damage is 2-phenylethanol, as its concentration in the air above a potato field allows correlation to the consumed leaf area (Schütz, 1997b). Only if cumulative damage surpasses an economic threshold does it make sense to apply control measures to avoid larger crop failure. Prophylactic chemical treatment can be avoided this way. 
Infestation by the Colorado potato beetles is initially punctual and spreads as the population increases over the whole field. A well-directed control measure against infestation hotspots is possible by the detection of (Z)-3-hexen-1-ol, a marker compound correlated with the ongoing leaf damage. Therefore, monitoring of 2-phenylethanol and (Z)-3-hexen-1-ol by a biosensor based on the antenna of the Colorado potato beetle situated at a field margin or on a tractor enables the spatial assessment of cumulative and recent damage by taking wind direction and wind speed into account. This can result in on-site recommendations for efficient and economic pest management (Schütz and Weißbecker, 2003).

\subsubsection{Assessment of Increased Infestation Disposition for Insect Forest Pests}

The change to alternative energy sources such as renewable primary products (e.g., timber) has led to a focus on intensified utilization of our forests. As the value of timber increases its harvesting implies a profit gain and this consequently requires effective management systems. When land usage is intensified in forest stands possible risks have to be considered, for example nutrient loss, drought stress, or sunburn. These factors primarily lead to a decrease of accrescence and debilitation of the trees. A secondary effect is, for instance, the attraction of insects by the modified scent of debilitated trees. As the defense capability of such trees is lowered the survival rate of the insect's offspring rises. This leads to an increase in the pest's population, which is then able to infest intact trees, as well. Such complex ecological interactions can cause high economic losses. Therefore, a biosensor system on the basis of a pest species antenna to detect debilitated trees would enable early retaliatory actions.

The blue pine jewel beetle Phaenops cyanea is able to distinguish between short but vitality-decreasing drought stress, for instance on pine, and stress inflicted by fungi infestation or mechanical damage, for example via storms. It utilizes the fact that pines emit different scents when under drought and when stressed by fungi or mechanical damage (Schütz et al., 2004) and it is able to recognize the different marker compounds. These marker compounds are even detected in the ppb range (Schroth et al., 2001) and therefore, can be traced over long distances to damaged pine stands. Consequently, the antenna of the blue pine jewel beetle has the required sensitivity and selectivity when integrated into a biosensor setup. In order to detect long-term drought stress the antenna of the longhorn beetle Monochamus galloprovincialis, which is able to recognize selectively cyclic and oxidized monoterpenes emitted by trees under such stress situations, can be utilized (Weißbecker et al., 2006). For a practically relevant determination of whether a tree stand is damaged seriously enough to expect an infestation by $P$. cyanea, quantitative information correlated to the degree of damage is required. The marker compounds 
(Z)-3-hexen-3-ol and nonanal are correlated with the degree of cell-decomposition in the bast of the trees. The detection of these marker compounds in a pine stand enables one to conclude the degree of damage of the assimilate-conducting tissue and thereby, the predisposition for an infestation by P. cyanea (Schütz et al., 2004).

\subsubsection{Post Mortem Interval (PMI) Estimation in Legal Medicine}

In legal medicine the colonization of corpses by necrophagous insects is used to estimate the time elapsed since death. Different insect species colonize a dead vertebrate in waves, each associated with distinct stages of decay. Studies on a vertebrate's volatile profile in a certain state of decay have shown that there is a high variation in the qualitative scent composition (Statheropoulos et al., 2005, 2007; Dekeirsschieter et al., 2009). To investigate any constantly occurring trace compounds during vertebrate decay on the basis of trace analysis would probably exceed the realistic limits of manual data acquisition. Insect species can be utilized in post mortem interval estimation to avoid significant expense in regards to time and money as they have already overcome the problem of volatile pattern variation. For instance, in a GCMS/EAD setup the odor compound selection of several insects associated with early and later stages of decay are analyzed to reveal marker compounds that are typically emitted in these stages. According to the odor selection there are basically two types of carrion-colonizing insects, a general type that occurs during most of the decay period and a specialized type that occurs only at distinct stages of decomposition. Both types are valuable when a significant marker compound dynamics leading to a post mortem interval estimation is required. The former type can be used to develop a biosensor to indicate and locate the presence of corpses, while the latter type can provide quantitative patterns distinct for earlier or later stages of decay. The comparative relative quantity of compounds can lead to a dynamic marker compound pattern as a common core of the scent of decay. A suitable species for discovering such marker compound patterns may be, for instance, Calliphora vicina, a bluebottle blowfly species, as it occurs shortly after death until advanced decay (Smith, 1986). Odor compounds filtered by this fly species are-for example sulfur compounds like dimethyl-trisulfide (Stensmyr et al., 2002) — general compounds emitted during various metabolic activities. By utilizing the antennae of this fly species in a biosensor setup it is possible not only to estimate the post mortem time, but also to trace corpses or victims of natural disasters, for example after earthquakes. Insect antennae of specialized types of necrophagous insects should be selective for the identification of marker compounds characterizing certain stages of decay. For instance, there should be an increase of compounds associated with fermentation during the bloated stage and an increase in compounds associated with aerobic metabolic activity during the successive stage where the skin ruptures and the inner matter comes into contact with air. Combining the odor selection of both types of necrophagous insect antennae could lead to a biosensor setup that enables the estimation of post mortem interval as well as the tracing of corpses. 


\subsection{Biomimetic Approaches to Sensors on the Basis of Insect Olfaction}

Analyzing odors emitted due to the metabolic activity of organisms adapted to an ecological context usually results in a very high qualitative and quantitative variation of the odor-constituting compounds. Despite this high variation insects are able to orientate towards the source of the scents by olfactory means. Taking into account recent knowledge of the biochemical chain of odor compound detection this implies that insects filter marker compounds from the whole scent emitted from the desired source, despite variation factors, such as environmental conditions (e.g., temperature) or metabolic activity of other organisms (e.g., microorganisms). Such marker compounds significantly display distinct conditions of dynamic ecological processes. However, prior to transferring them to the databases of functional sensor systems for monitoring these processes, limitations in the information content of the marker compounds as well as technical limitations have to be taken into account. Limitations in the information content of the marker compounds have to be considered according to the requirements of the technical sensor solution. A marker compound that significantly defines a natural process is not necessarily conferrable to an artificial industrial process or a processed industrial product. For instance, using the detection of a thermal combustion product as a marker for smoldering wood might not be significant for smoldering wood that has been impregnated. Regarding the emission rate of compounds, the infestation of natural wood by fungi should lead to quantitatively different emission progress compared to infestation of chemically treated wood. Although nature has provided an approach towards the solution of such cases, for example early fire detection utilizing the odor filtering abilities of $M$. acuminata, adaptation to alterations of natural processes is required.

Considering the performance of biosensors on the basis of insect antennae it is not possible yet to mimic its high sensitivity and selectivity in detecting single compounds by an artificial solely technical device. Anyway, progress in gas sensor applications provides devices with certain advantages in comparison to biosensors. The major drawback of the latter is the short life-time of the antenna which restricts its efficient usage. Although the requirements of running a biosensor are not high, there is a requirement for personnel to operate the systems.

Thus, biosensors are more useful for on-site screening or tracking purposes than for long-term monitoring purposes. In contrast solid-state sensors like semiconductor gas sensors have a lifetime of approximately 1 year, which makes them good candidates for low-cost monitoring applications in industrial processes. As they do not possess the selectivity and sensitivity of biosensors their applicability is restricted concerning the concentration of the compounds to be traced and the level of identification, which is rather compound groups, like aldehydes or amines as opposed to single compounds. Applications of semiconductor gas sensors include, for instance, monitoring the shelf-life of fish (Nicolay, 2006), monitoring of wheat for fungal contamination (Presicce, 2006), detecting different mold species in buildings (Kuske, 2006), and smoldering fires in coal storage units (Schütz et al., 1999) or the discrimination of toasted or untoasted bread (Bock et al., 1997). To combine this 
technique with the concept of marker compounds is quite challenging, as the sensor has to be modified to respond selectively and to gate out any other compound reactions. This can be achieved with semiconductor gas sensor arrays where the combined reactions of all sensors in the array lead to a compound-specific pattern. Moreover, sensor filters, sensor surface modifications, temperature cycles, algorithm analysis, or artificial neuronal networks (ANN) serve to improve the sensors performance. As the efficient traceability of marker compounds is a valuable aim, the potential for enhancements in this field of technological research is high in order to successfully utilize the blue prints from nature. For instance, as outlined above, the amount of 2-phenylethanol emitted by potato plants or cyclic and oxidized monoterpenes emitted by trees under drought stress can be used to determine the ecological status of the field or the tree. The following examples describe further possible applications of biomimetic semiconductor gas sensor systems in industry.

\subsubsection{Detection of Meat Spoilage}

In our modern food industry quality control is the most important link between producer and customer. Trust is a strong marketing factor, but due to several scandals in recent times it became obvious, that the producer is not always able to guarantee a minimum quality. Especially in the meat industry this is still a problem. Therefore, there is a strong need for innovation in this field.

Generally, any cells including muscle cells contain fat due to phospholipid membranes. Fatty acids, like linolic acid or arachidonic acid are typically abundant in membrane lipids and can be degraded to volatile aliphatic alcohols and aldehydes. Intra-muscular fat consists of fat cells inside the muscular tissue. Because of a permanent turnover in all tissues, the composition of nutrition has a strong influence on the composition of the triacylglycerides. The second important source of meat volatiles are proteins. The amino acid concentration of muscle tissue shows variations between species of animals (Branscheid et al., 2007). Amino acid chains in meat tissue are mainly assembled to form the basic functional unit of the myosin-actin complex that enables neuron-induced movement.

As the basic function of this complex is the same in all vertebrates, it is supposed that its biochemical properties and therefore, the amounts of different amino acids are the same, as well. Equal to protein-induced lipid degradation processes, meat tissue contains proteolytic enzymes that cleave muscle proteins post mortem. The main changes take place by fragmentation of myofibrils through the z-disk, degradation of desmin, nebulin, and titin and the appearance of two polypeptides with a molecular mass of 95 and $30 \mathrm{kDa}$. These phenomena are most likely induced by calpains and cathepsins. Putrescine, cadaverine, tyramine, and histamine are products of bacterial metabolism and E. coli play an important role in the formation of histamine and cadaverine (Durlu-Özkaya et al., 2001). The variability of biogenic amines occurring during fermentation processes of meat is very high and depends on the microorganisms growing on the tissue. As these amines are not volatile under moderate temperature conditions a further degradation is needed 
to get a protein-induced flavor. Sulfur compounds are volatiles usually emitted by decaying meat (Mayr et al., 2003), fermented meat (Barbieri et al., 1992), and also cooked meat (Shahidi, 1998). It is most likely that these volatiles have their origin in methionine and cysteine, which are the major sulfur-containing compounds in meat tissue.

There has been some effort to correlate the volatiles emitted by decaying meat to its grade of spoilage (Stutz et al., 1991; Winquist et al., 1993; Nicolay, 2006; Mayr et al., 2003). These efforts resulted in sensor concepts disregarding the high diversity of the scent of decay, which limits their application range (Winquist et al., 1993; Nicolay, 2006).

As $C$. vicina is an organism associated with the colonization of vertebrate matter shortly after death it should be able to detect compounds emitted in the early phase of decomposition. These compounds can be regarded as marker compounds for the early spoilage of vertebrate tissue. Thus, by adapting a semiconductor gas sensor system to the dynamic marker compound pattern of aging meat it is possible to estimate the freshness of meat. Such a sensor system could be applied along the whole chain of custody from the slaughterhouse to the consumer. The most important restriction of such a sensor system is the detection threshold for the marker compounds as the emission rates in the early stages of spoilage are usually low.

\subsubsection{Early Fire Warning System in Wood Flake Driers}

The pyrophilic beetle $M$. acuminata has already been described above including its ability to detect fire over a long distance. Once it has reached the freshly burned area the female beetle oviposits on stems that were not completely destroyed by the fire. The hatching larvae feed on the cambium and later on the intact wood tissue. To be able to find an appropriate place for oviposition, the beetle has to be able to distinguish between different stages of heated or burned wood tissue. This ability can result in a marker compound pattern that significantly correlates the emission of compounds during wood heating with the temperature of the wood. This pattern, when detected by a semiconductor gas sensor system, can lead to fire warning applications that can detect wood fires before they ignite. Such applications can be adapted to, for instance, wood flake driers in the wood processing industry. Reducing the water content of flakes in order to glue and press them to boards requires a large energy input. As the drying process proceeds more efficiently at higher temperatures it would be more efficient to dry the flakes just under the threshold of self-ignition. Under production conditions the temperature is usually lowered to a level that ensures fire safety by monitoring the air temperature and installing spark extinguishing systems. Monitoring the marker compounds for heated wood just before the threshold of self-ignition would enable the temperature to be increased in wood flake driers without increasing the fire risk. This has the advantages of reducing energy consumption, the loss of material, and damage due to fire. Such systems can also be installed in biomass storage units, for 
example self-ignition of saw dust that is stored in silos as a renewable energy source is one of the problems faced by modern energy plants. Applications for wooden constructions, like warehouses, hotels, or private residential buildings are possible, although the relatively high energy consumption of semiconductor gas sensors limits commercial viability in the private market.

Acknowledgments Funding by the BMBF for "Biosensors on the basis of insect olfaction" and "BioHot" is gratefully acknowledged. Funding by the Deutsche Forschungsgemeinschaft (DFG) for "Multidimensional EAG" is gratefully acknowledged. S. P. is funded by the Cusanuswerk, Germany.

\section{References}

Badihi-Mossberg M, Buchner V, Rishpon J (2007) Electrochemical biosensors for pollutants in the environment. Electroanal 19:2015-2028

Barbieri G, Bolzoni L, Parolari G, Virgili R, Buttini R, Careri M, Mangia A (1992) Flavor compounds of dry-cured ham. J Agric Food Chem 40:2389-2394

Bock J, Kohl D, Schieberle P (1997) Discrimination between toasted and untoasted white bread. Sensors Ser, ISBN 83-908335-0-6, pp 863-866

Branscheid W, Honikel KO, v.Lengerken G, Troeger K (2007) Qualität von fleisch und fleischwaren. Deutscher Fachverlag GmbH, Frankfurt am Main

Clark LC, Lyons C (1962) Electrode systems for continuous monitoring in cardiovascular surgery. Ann N Y Acad Sci 102:29-45

Dekeirsschieter J, Verheggen FJ, Gohy M, Hubrecht F, Bourguignon L, Lognay G, Haubruge E (2009) Cadaveric volatile organic compounds released by decaying pig carcasses (Sus domesticus L.) in different biotopes. Forensic Sci Int 189:46-53

Durlu-Özkaya F, Ayhan K, Vural N (2001) Biogenic amines produced by Enterobacteriaceae isolated from meat products. Meat Sci 58:163-166

Hillson NJ, Hu P, Andersen GL, Shapiro L (2007) Caulobacter crescentus as a whole-cell uranium biosensor. Appl Environ Microbiol 73:7615-7621

Kaissling K-E, Priesner E (1970) Die Riechschwelle des Seidenspinners. Naturwissenschaften $54: 23-28$

Kuske M (2006) Detection of diverse mould species growing on building materials by gas sensor arrays and pattern recognition. Sens Actuators B Chem 118:1-2

Liu J, Mattiasson B (2002) Microbial BOD sensors for wastewater analysis. Water Res $36: 3786-3802$

Lowe CR (1985) An introduction to the concepts and technology of biosensors. Biosensors 1:3-16

Mayr D, Margesin R, Klingsbichel E, Hartungen E, Jenewein D, Schinner F, Mark TD (2003) Rapid detection of meat spoilage by measuring volatile organic compounds by using proton transfer reaction mass spectrometry. Appl Environ Microbiol 69:4697-4705

Nicolay X (2006) Odors in the Food Industry. Springer, New York, NY

Presicce DS (2006) Response evaluation of an E-nose towards contaminated wheat by Fusarium poae fungi. Sens Actuators B Chem 118:1-2

Rodriguez-Mozaz S, Lopez de Alda MJ, Barceló D (2006) Biosensors as useful tools for environmental analysis and monitoring. Anal Bioanal Chem 386:1025-1041

Rudolph AS, Reasor J (2001) Cell and tissue based technologies for environmental detection and medical diagnostics. Biosens Bioelectron 16:429-431

Rützler M, Zwiebel LJ (2005) Molecular biology of insect olfaction: recent progress and conceptual models. J Comp Physiol A 191:777-790

Scheller FW, Schubert F, Renneberg R, Müller H-G, Jänchen M, Weise H (1985) Biosensors: trends and commercialization. Biosensors 1:135-160 
Schroth P, Schöning MJ, Lüth H, Weißbecker B, Hummel HE, Schütz S (2001) Extending the capabilities of an antenna/chip biosensor by employing various insect species. Sens Actuators B Chem 78:1-5

Schöning MJ, Schütz S, Schroth P, Weißbecker B, Steffen A, Kordos P, Lüth H, Hummel HE (1998) A BioFET on the basis of intact insect antennae. Sens Actuators B Chem 47:234-237

Schügerl K (1985) Sensor-Meßtechniken in der biotechnologischen Forschung und Industrie. Naturwissenschaften 72:400-407

Schütz S, Weißbecker B (2003) Mechanismen duftvermittelter Pflanze-Insekt-Interaktionen: vom Pflanzenstreß zum Pflanzenfraß. Nova Acta Leopold NF 87(328):215-235

Schütz S, Weißbecker B, Apel K-H, Wenk M (2004) Duftstoffsignale als Marker für die Befallsdisposition von Kiefern durch den Blauen Kiefernprachtkäfer Phaenops cyanea F (Col., Buprestidae). Mitt Dtsch Ges Allg Angew Entomol 14:301-306

Schütz S, Weißbecker B, Hummel HE, Apel K-H, Schmitz H, Bleckmann H (1999) Insect antenna as a smoke detector. Nature 398:298-299

Schütz S, Weißbecker B, Hummel HE, Schöning MJ, Riemer A, Kordos P, Lüth H (1997a) Field effect transistor-insect antenna junction. Naturwissenschaften 84:86-88

Schütz S, Weißbecker B, Klein A, Hummel HE (1997b) Host plant selection of the Colorado potato beetle as influenced by damage induced volatiles of the potato plant. Naturwissenschaften $84: 212-217$

Shahidi F (1998) Flavour of meat, meat products and seafoods, 2nd edn. Blackie Academic \& Professional, London

Smith KGV (1986) A manual of forensic entomology. Department of Entomology, British Museum (Natural History), London

Statheropoulos M, Agapiou A, Spiliopouiou C, Pallis GC, Sianos E (2007) Environmental aspects of VOCs evolved in the early stages of human decomposition. Sci Total Environ 385:221-227

Statheropoulos M, Spiliopouiou C, Agapiou A (2005) A study of volatile organic compounds evolved from the decaying human body. Forensic Sci Int 153:147-155

Stensmyr MC, Urru I, Collu I, Celander M, Hansson BS, Angioy AM (2002) Rotting smell of dead-horse arum florets - these blooms chemically fool flies into pollinating them. Nature 420:625-626

Stutz HK, Silverman GJ, Angelini P, Levin RE (1991) Bacteria and volatile compounds associated with ground-beef spoilage. J Food Sci 56:1147-1153

Tegoni M, Campanacci V, Cambillau C (2004) Structural aspects of sexual attraction and chemical communication in insects. Trends Biochem Sci 29:257-264

Theegala CS, Small DD, Monroe WT (2008) Oxygen electrode-based single antibody amperometric biosensor for qualitative detection of E. coli and bacteria in water. J Environ Sci Health A 43:478-487

Tokarskyy O, Marshall DL (2008) Immunosensors for rapid detection of Escherichia coli O157: H7 - Perspectives for use in the meat processing industry. Food Microbiol 25:1-12

Turner A, Zourob MM, Elwary S (2008) Handbook of biosensors. Microsystems for bacterial detection. Springer, New York, NY

Vogt RG, Riddiford LM (1981) Pheromone binding and inactivation by moth antennae. Nature 293:161-163

Weißbecker B, Holighaus G, Schütz S (2004) Gas chromatography with mass spectrometric and electroantennographic detection: -analysis of wood odour by direct coupling of insect olfaction and mass spectrometry. J Chromatogr A 1056:209-216

Weißbecker B, Schröder T, Apel K-H, Schütz S (2006) Perception of host odours by forest pests: comparison of a wood breeding beetle (Monochamus galloprovincialis) and a bark breeding beetle (Phaenops cyanea). Mitt Dtsch Ges Allg Angew Entomol 15:235-238

Wicher D, Schafer R, Bauernfeind R, Stensmyr MC, Heller R, Heinemann SH, Hansson BS (2008) Drosophila odorant receptors are both ligand-gated and cyclic-nucleotide-activated cation channels. Nature 452:1007

Winquist F, Hornsten EG, Sundgren H, Lundstrom I (1993) Performance of an electronic nose for quality estimation of ground meat. Meas Sci Technol 4:1493-1500 


\title{
Post-mortem volatiles of vertebrate tissue
}

\author{
Sebastian Paczkowski • Stefan Schütz
}

Received: 24 May 2011 /Revised: 24 May 2011 / Accepted: 24 May 2011

(C) The Author(s) 2011. This article is published with open access at Springerlink.com

\begin{abstract}
Volatile emission during vertebrate decay is a complex process that is understood incompletely. It depends on many factors. The main factor is the metabolism of the microbial species present inside and on the vertebrate. In this review, we combine the results from studies on volatile organic compounds (VOCs) detected during this decay process and those on the biochemical formation of VOCs in order to improve our understanding of the decay process. Micro-organisms are the main producers of VOCs, which are by- or end-products of microbial metabolism. Many microbes are already present inside and on a vertebrate, and these can initiate microbial decay. In addition, microorganisms from the environment colonize the cadaver. The composition of microbial communities is complex, and communities of different species interact with each other in succession. In comparison to the complexity of the decay process, the resulting volatile pattern does show some consistency. Therefore, the possibility of an existence of a time-dependent core volatile pattern, which could be used for applications in areas such as forensics or food science, is discussed. Possible microbial interactions that might alter the process of decay are highlighted.
\end{abstract}

Keywords Post-mortem decay. Post-mortem volatiles . Decaying vertebrates . Volatile emission $\cdot$ Biochemical volatile formation - Volatiles of microbial metabolism

\footnotetext{
S. Paczkowski $(\bowtie) \cdot$ S. Schütz

Department of Forest Zoology and Forest Conservation, Büsgeninstitut, Georg August University,

Büsgenweg 3,

37077 Göttingen, Germany

e-mail: spaczko@gwdg.de
}

Published online: 01 July 2011

\section{Introduction}

A vertebrate is a complex structure of chemically interacting compounds. Some functional features like muscle contraction are constant in all vertebrates. Phospholipids in cell membranes, actin and myosin in muscle cells, or collagen and hydroxyapatite in bones are chemical structures that are indispensible in the biology of a vertebrate and exist in all vertebrates. The genetic makeup and diet have an impact on the quantitative chemical composition of a vertebrate. In muscle proteins, the relationship of different amino acids varies between species (Branscheid et al. 2007) and the quantitative composition of bones is influenced by both the diet (Sos et al. 1961) and species (Ravaglioli et al. 1996). The fat content, such as the composition and abundance of fatty acids, triglycerides, phospholipids, and steroids, shows high variability (Gurr 1988), and it is influenced by genetic makeup (Branscheid et al. 2007;Estevez et al. 2003; Insausti et al. 2005; Sink 1979a). The fat composition of the diet also directly influences the vertebrate's fat composition (Lee et al. 2004; Sink 1979b). These results indicate that vertebrates contain chemical structures of low and high variability.

Consumers or destruents are heterotrophic organisms in the food chain that are able to feed on cadavers. Consumers are adapted to hunt vertebrate prey but can also feed on cadavers and, besides other sources, destruents can feed on dead vertebrates as well. A dead vertebrate has a complex and time-dependent faunal succession composed of birds (Moreno-Opo et al. 2010), omnivorous mammals (Haglund 1997), and insects (Amendt et al. 2004;Smith 1986). There exist very little experimental literature on the microbiological succession on dead vertebrates (Hopkins et al. 2000). Microorganisms are present in all the geographical regions of the world and play the important role of recyclers in ecosystems. They degrade organic matter to inorganic substances, which 
are then made available to primary producers. The chemical reactions catalyzed by microbial enzymes yield volatile organic compounds (VOCs) as the by- or end-products. Micro-organisms can adapt their metabolism to their habitat. This adaption depends on many factors such as the complex and variable chemical composition of the decomposing dead vertebrate, temperature of the habitat, or competition between different microbial species (Kai et al. 2009). Consequently, the pattern of volatiles released during vertebrate decay can vary. The aim of this review is to summarize the volatiles whose presence has been consistently reported during the process of decay and to link these volatiles to their processes of formation. Based on the results, we discuss possible applications of these volatile patterns to forensics and the detection of food spoilage.

\section{Post-mortem decay initiated by endogenous enzymes}

Immediately after death, the heart activity ceases and the lack of oxygen leads to considerable physiological changes. Glycogen is degraded to lactic acid in the skeletal muscle (Branscheid et al. 2007). This decreases the $\mathrm{pH}$ during the first hours post-mortem. In the case of high muscle activity before death, this effect can occur even minutes after death because the muscle already lacks oxygen. Another consequence is the cessation of pyruvate transport across the mitochondrial membrane. The oxidation of pyruvate to acetyl-CoA stops, and acetyl-CoA cannot enter the citrate cycle. The respiratory chain requires the low electronegativity of oxygen because it generates the electron gradient necessary for ATP production. As ATP is a universal energy source in vertebrate cells, the termination of its anabolism leads to the cessation of regular metabolism. One of the consequences of low ATP levels is the binding of the myosin-actin complex in the muscle, resulting in rigor mortis. Another effect is the inability of the cells to repair their own membranes. Consequently, lysosomal hydrolytic enzymes are released in an uncontrolled fashion in vertebrate cells (Ito et al. 1991a, b). The proteolytic enzymes released include lysosomal cathepsins and calcium-dependent endopeptidases in the muscle cells. Endopeptidases mainly consist of calpain I for low calcium levels shortly after death and calpain II for high calcium levels. High calcium levels result from increasing influx due to the onset of decay of longitudinal tubuli in the muscle cells. These proteolytic enzymes fragment the myofibrils of muscle cells along the z-disk. They react with troponins $\mathrm{T}$ and $\mathrm{I}$, tropomyosin, C-protein, filamin, vinculin, desmin, titin, and nebulin (Dransfield 1992; Goll et al. 1983; Koohmaraie 1992, 1994). Toldra (1998) reported lipase and phospholipase activity inside fermented ham, although halophilic microorganisms were only abundant on the surface. This suggested that the activity of lipolytic enzymes arose from the muscle tissue itself. Furthermore, lipoprotein lipase is present in the skeletal muscles of rats and hydrolyses di- and triglycerides of low-density lipoproteins at an optimum $\mathrm{pH}$ value of 8 to 9 (Miller et al. 1987;Motilva et al. 1993; Okuda 1991). Lysosomal acid lipase was found in the human liver, placenta, aorta, and leukocytes (Sando and Rosenbaum 1985), and it hydrolyses lipids at a pH optimum of 4 to 5 (Motilva et al. 1993; Negre et al. 1985). This suggests that the activity of lipoprotein lipase ceases shortly after death due to lactic acid accumulation. The low $\mathrm{pH}$ suppresses lipoprotein lipase and supports the activity of lysosomal acid lipase. The phospholipids of the cell membranes are degraded by phospholipases (Toldra 1998). Autolysis of arachidonic acid by the skin enzyme system of ayu fish (Plecoglossus altivelis) yields 2-octenal, 1-octen-3-ol, 2nonenal, 2-octen-1-ol, and 3-nonen-1-ol, and the autolysis of eicopentaenoic acid yields 1,5-octadien-3-ol, 2,6-nonadienal, 2,5-octadien-1-ol, and 3,6-nonadien-1-ol (Zhang et al. 1992). In an anaerobic environment, fatty acids can remain in adipoceres in certain circumstances. Several studies have examined adipocere formation under artificial (Forbes et al. 2005a, b, c; Yan et al. 2001) and field (Forbes et al. 2002; Gill-King 1999) conditions.

In general, lysosomal hydrolytic enzymes are active at an optimum temperature of $37^{\circ} \mathrm{C}$ (Dutson 1983). Most of these enzymes are also active at lower temperatures but have lower activity. For example, some cathepsins are active at $25{ }^{\circ} \mathrm{C}$, and $\mathrm{Ca}^{2+}$-dependent proteinase $(\mathrm{CAF})$ is responsible for proteolysis in meat stored at $0-4{ }^{\circ} \mathrm{C}$ (Goll et al. 1983). Another example is calpastatin, which inhibits calpains with $87 \%$ of the maximum rate at $25{ }^{\circ} \mathrm{C}$ and $\mathrm{pH}$ 7.5. Temperature and enzyme activity are correlated in a non-linear way (Goll et al. 1983).

Reactive oxygen species (ROS) are formed enzymatically, chemically, and photochemically. They react with lipids, amino acids, and sugars. Oxidized lipids release volatile aldehydes, alcohols, or alkenes. Oxidized methionine forms $\mathrm{H}_{2} \mathrm{~S}$, while oxidized sugars form ketones (Choe and Min 2005). Living vertebrates compensate the cleavage reactions of ROS by repair processes or quench these highly reactive molecules by antioxidant defense mechanisms (Cadenas 1989). After death, these repair mechanisms cease, and ROS product emission increases. This increase might be the first sign of a postmortem VOC pattern. During the active and advanced stages of decay, these emissions play a minor role in comparison to the volatiles produced by microbial activity.

\section{Post-mortem decay of vertebrates by micro-organisms}

The microbial decay of a vertebrate is a dynamic process that depends on a highly diverse assembly of microbial species, 
their interactions (Kai et al. 2009; Stams 1994), and the climatic conditions of the habitat. Temperature has a strong effect on the microbial species composition. For instance, psychrotrophic or mesophilic bacteria exhibit optimal growth at different temperatures and also release different volatiles (Ercolini et al. 2009). Burial (Dent et al. 2004; Vass et al. 2008) and drowning or exposition (Dekeirsschieter et al. 2009; Statheropoulos et al. 2005) influence the microbial species composition. In general, the decay of a vertebrate is a process that occurs in different stages (Amendt et al. 2004; Dekeirsschieter et al. 2009) and can lead to complete decomposition or conservation, such as mummification. In the forensic literature, the decay of a vertebrate is often described in stages. The stage approach is a useful simplification of the decaying process as it is possible to characterize every stage of decay by its typical characteristics. Using this distinction, other phenomena like the infestation of the dead vertebrate with insect larvae can be described (Smith 1986). However, Matuszewski et al. found that a decomposing vertebrate can simultaneously exhibit different stages of decomposition (Matuszewski et al. 2010a) and Carter et al. states that the stages approach is an accepted and useful simplification but that the decaying process cannot be divided in discrete subunits (Carter et al. 2007). Therefore, most of the studies on volatiles of vertebrate decay (Table 3) don't mention the stage of decay in which the volatiles occurred. Recently, alternative methods for correlating postmortem interval (pmi) and decompositional process were proposed (Michaud and Moreau 2011).

Despite their destructive functions as destruents, microorganisms play an important role in living vertebrates by supporting the latter's vital functions. The number of microorganisms in the human gut was estimated to be in excess of $10^{11}$ cells/g gut contents (Moore and Holdeman 1974). Many facultative or obligate anaerobic species such as Escherichia coli and Clostridium perfringens are present in the healthy intestine where they metabolize predigested nutrients entering from the stomach (Corry 1978). Micro-organisms can adapt their metabolism to the diet of the vertebrate. The relative amounts of ammonia emitted, fecal volatile sulfur compounds, phenol and urinary 4-methylphenol in human feces depend on the relative proportion of meat in the diet of the vertebrate (Cummings et al. 1979; Geypens et al. 1997). Intestinal micro-organisms prefer different substrates such as carbohydrates or amino acids as their nutrition source or depend on the metabolic products of other microbes (Smith and Macfarlane 1996). Various microbes are present on mucous membranes, sexual organs and the skin (Jenny 1983; Labows 1982; Straehlerpohl and Exner 1985). Yeasts and bacteria may be present ante-mortem in the lymphatic and vascular system as a result of infection or uptake during nutrition (Chang and Kollman 1989; Wells et al. 1988). After death, the micro-organisms present begin to colonize the body and disperse in an uncontrolled manner from their original location. The infestation of local tissues adjacent to the intestine and the capillaries of the lymphatic or vascular system characterize the beginning of this migration. A second important source of microbes is the mucous membrane of the respiratory system. This microbial community consists of Staphylococcus aureus, some Streptococcaceae, Neisseria catarrhalis, Corynebacterium xerosis, Coryne. pseudodiphtheriticum, the yeast species Candida albicans and many other microbial species. In guinea pigs, it was reported that the immunosystem could neutralize spreading microbes during the first $24 \mathrm{~h}$ after death (Gill et al. 1976). This led to the hypothesis that after death, ROS oxidation and autolysis dominate decay until the immunosystem fails and microbial growth increases. Of course, the number of microbes mentioned here is limited to only a few examples. For more detailed information, see Ercolini et al. (2009) and Corry (1978).

\section{Carbohydrates}

The main substrates for microbial growth offered by a vertebrate are water, carbohydrates, amino acids, fats and minerals (Dent et al. 2004). Carbohydrates are the most readily utilized nutrition source for both microbes and mammals (Boumba et al. 2008). The Embden-MeyerhofParnas (EMP) glycolytic pathway and the Entner-Doudoroff (ED) pathway yield pyruvate from glucose. Pyruvate is fermented to ethanol and acetic acid by bacteria and yeasts (Boumba et al. 2008; Gottschalk 1986). Other by-products of this fermentation are pyruvic acid, lactic acid, butanoic acid, propanoic acid, acetaldehyde, acetone, propan-1-ol, propan2-ol, butan-1-ol and butane-1,3-diol (Boumba et al. 2008; Dent et al. 2004; Waksman and Starkey 1931). Incomplete aerobic degradation of sugar monomers by fungi yields organic acids such as glucuronic acid, citric acid and oxalic acid. Complete aerobic decay of sugar monomers results in carbon dioxide and water.

Clostridiaceae are obligate anaerobic bacteria that are present in the intestine and in anaerobic soil layers. Since they sporulate and can therefore survive under aerobic conditions, these bacteria can colonize a dead vertebrate from inside and from the external environment. This family is represented by species such as Clostridium perfringens (welchii), C. litus-eburense (Corry 1978; Haagsma 1991) and C. paraputrificum (Corry 1978). Many clostridiaceae species ferment pyruvate formed from carbohydrates to acetone, ethanol, butan-1-ol, acetic acid, butanoic acid or butane-1,3-diol (Boumba et al. 2008). Enterobacteriaceae such as $E$. coli are facultative anaerobes that are present in the intestine. E. coli ferments the pyruvate formed by glycolysis by mixed acid fermentation (Table 1) to lactic acid, succinic acid, acetic acid, formic acid and ethanol 
Table 1 Products of carbohydrate-utilizing microbes in a facultative anaerobic environment

\begin{tabular}{|c|c|c|c|}
\hline Pathway & Microbial families and species & Location & Metabolic products \\
\hline \multirow[t]{6}{*}{ Mixed acid fermentation } & Enterobacteriaceae & Intestine & $\begin{array}{l}\text { Lactic acid, succinic acid, } \\
\text { acetic acid, formic acid, ethanol }\end{array}$ \\
\hline & Escherichia coli & & \\
\hline & Enterococcaceae & $\begin{array}{l}\text { Oral cavity, intestine, } \\
\text { urethra, sexual organs }\end{array}$ & $\begin{array}{l}\text { Lactic acid, succinic acid, } \\
\text { acetic acid, formic acid, ethanol }\end{array}$ \\
\hline & $\begin{array}{l}\text { Enterococcus faecalis, } \\
\text { Enterococcus faecium }\end{array}$ & & \\
\hline & Bacillaceae & Upper soil layers & $\begin{array}{l}\text { Lactic acid, succinic acid, acetic acid, } \\
\text { formic acid, ethanol, butane-2,3-diol }\end{array}$ \\
\hline & Bacillus subtilis & & \\
\hline \multirow[t]{3}{*}{ Lactic acid fermentation } & Streptococcaceae & Oral cavity & Acetic acid, ethanol \\
\hline & Lactobacillaceae & Intestine & Lactic acid, ethanol \\
\hline & Lactobacillus acidophilus & & \\
\hline 2,3 Butanediol fermentation & $\begin{array}{l}\text { Klebsiella pneumoniae } \\
\text { subsp. pneumoniae }\end{array}$ & Intestine & $\begin{array}{l}\text { Butane-2,3-diol, lactic acid, } \\
\text { acetic acid, formic acid, ethanol }\end{array}$ \\
\hline \multirow[t]{2}{*}{ Propanoic acid fermentation } & Propionibacteriaceae & Intestine, skin & Propanoic acid, acetic acid \\
\hline & Propionibacterium acnes & & \\
\hline \multirow[t]{2}{*}{ Bifidum pathway } & Bifidobacteriaceae & Intestine, sexual organs & Lactic acid, acetic acid \\
\hline & Bifidobacterium animalis & & \\
\hline
\end{tabular}

(Boumba et al. 2008). It can switch from glycolysis to lactose utilization to form pyruvate, which is then transformed to the volatile products acetic acid and ethanol. The genus Klebsiella of the family enterobacteriaceae is a gramnegative, facultative anaerobe that lives in soil, water or grain. Klebsiella pneumoniae subsp. pneumoniae is the only species of this genus known to be abundant in the intestine of vertebrates. Among intestinal enterobacteriaceae, this species is characterized by its use of butane-2,3-diol fermentation for energy production. Butane-2,3-diol, lactic acid, acetic acid, formic acid and ethanol are the main endproducts of butane-2,3-diol fermentation (Gottschalk 1986). Staphylococcus aureus from the family Staphylococcaceae, formerly a member of Micrococcaceae, is a bacterium that is abundant in the soil, skin and respiratory system of vertebrates. Streptococcaceae are facultative anaerobes that are abundant in the oral cavity where they ferment carbohydrates to lactic acid (Table 1). The facultative anaerobic family enterococcaceae ferments carbohydrates to lactic acid by mixed acid fermentation. Members of this family such as Enterococcus faecalis and Enterococcus faecium are present in the oral cavity, intestine, urethra and sexual organs. Due to their lack cytochromes and porphyrins, these organisms are aero-tolerant. Propionibacteriaceae such as Propionibacterium acnes are aero-tolerant anaerobic species that grow relatively slowly on the skin and in the intestine of vertebrates. These bacteria can ferment carbohydrates or lactic acid to propanoic acid and acetic acid by propanoic acid fermentation (Table 1). Species of the family lactobacillaceae such as Lactobacillus acidophilus, which grows at a $\mathrm{pH}$ of 4-5 or lower, are facultative anaerobes or obligate anaerobes present in the intestine of vertebrates. They produce lactic acid (homofermentive) or lactic acid, acetate and ethanol (heterofermentive) by lactic acid fermentation. Bifidobacteriaceae such as Bifidobacterium animalis are anaerobic bacteria present in the intestine or sexual organs of vertebrates where they metabolize carbohydrates by the bifidum pathway to form lactic acid and acetic acid (Table 1). The contribution of yeasts to vertebrate decay is less than that of bacteria (Corry 1978). One yeast species reported to be present on decaying vertebrates is Candida sp., especially Candida albicans (Chang and Kollman 1989; Corry 1978), which is present in the intestine, on the mucous membranes of the oral cavity and the genitals and between fingers and toes. Saccharomyces cerevisiae (Corry 1978; Davis et al. 1972), another yeast species, is a facultative aerobe that obtains energy by respiration or fermentation.

The constant inner putrefaction caused by these microorganisms and other microbes leads to the formation of organic and inorganic gases that cause bloating. The putrefaction of the cadaver that follows results in the breakage of the outer skin. Consequently, the inner fluid comes into contact with oxygen and aerobic species from the air and soil, which then colonize it. Aerobic bacillaceae species, mainly Bacillus subtilis, which is abundant in the upper soil layers, can adapt to facultative anaerobic conditions by switching to mixed acid fermentation of sugars. This microbe grows faster under aerobic 
conditions. Pseudomonaceae are abundant in soil, water and on the skin of vertebrates. They are aerobic and cannot ferment. For energy production, they utilize the Entner-Doudoroff pathway, which yields pyruvate predominantly from hexoses. These microbes can also generate the electron gradient of the respiratory chain by reducing nitrate to nitrogen instead of reducing oxygen. Neisseriaceae such as Neisseria catarrhalis are aerobic species that are abundant on the mucosal membranes of vertebrates. Corynebacteriaceae are aerobic or facultative anaerobic bacteria, and several species such as Corynebacterium xerosis or Coryne. pseudodiphtheriticum inhabit the mucosal membranes or skin of vertebrates.
Amino acids

In all vertebrates, amino acids are the major components of muscle tissue proteins, membrane proteins and free proteins. Although the functional mechanisms of proteins are complex and diverse, they are composed of the same amino acids. Microbial proteases and peptidases yield free amino acids. Amino acid degradation can also lead to the formation of volatile products (Table 2).

Durlu-Özkaya et al. (Durlu-Özkaya et al. 2001) stated that putrescine, cadaverine, tyramine and histamine in the skeletal muscle are products of proteolytic bacterial metabolism and that $E$. coli plays an important role in the

Table 2 Metabolic products of amino acid degradation

\begin{tabular}{|c|c|c|}
\hline \multirow[t]{2}{*}{ Leucine } & Ehrlich pathway, anabolism & $\begin{array}{l}\text { Propan-1-ol, 2-methyl-propan-1-ol, } \\
\text { 2-methyl-butan-1-ol, 3-methyl-butan-1-ol }\end{array}$ \\
\hline & M. phenylpyruvica, S. xylosus, S. starnosus & $\begin{array}{l}\text { 3-Methyl-butan-1-ol, 3-methyl-butanal, } \\
\text { 3-methyl-butanoic acid }\end{array}$ \\
\hline \multirow[t]{2}{*}{ Isoleucine } & Ehrlich pathway, anabolism & $\begin{array}{l}\text { 1-Propanol, 2-methyl-propan-1-ol, } \\
\text { 2-methyl-butan-1-ol, 3-methyl-butan-1-ol }\end{array}$ \\
\hline & Yeast & $\begin{array}{l}\text { Propan-1-ol, 2-methyl-butan-1-ol, } \\
\text { 3-methyl-butan-1-ol, pentanol }\end{array}$ \\
\hline Threonine & Yeast & $\begin{array}{l}\text { 1-Propanol, 2-methyl-1-butanol, } \\
\text { 3-methyl-1-butanol, pentan-1-ol }\end{array}$ \\
\hline Arginine $>$ ornithine & Ornithine decarboxylase & Putrescine \\
\hline Lysine & Lysine decarboxylase & Cadaverine \\
\hline \multirow[t]{3}{*}{ Tyrosine } & $\begin{array}{l}\text { S. albus, B. fragilis, Fusobacterium sp., } \\
\text { Bifidobacterium spp., C. paraputrificum, } \\
\text { C. butyricum, C. sporogenes, C. septicum }\end{array}$ & 4-Methylphenol (anaerobe) \\
\hline & & $\begin{array}{l}\text { 2-Phenylethanol, phenylacetaldehyde, } \\
\text { phenylacetic acid }\end{array}$ \\
\hline & E. coli, Proteus sp., E. faecalis, S. albus & Phenol (facultative anaerobe) \\
\hline \multirow[t]{6}{*}{ Phenylalanine } & Phenylalanine decarboxylase $+\mathrm{Fe}^{3+}$ & Green complex \\
\hline & Pseudomonaceae (aerobe) & $\begin{array}{l}\text { 2-Phenylethanol, phenylacetaldehyde, } \\
\text { phenylacetic acid }\end{array}$ \\
\hline & & Phenylpropanoic acid \\
\hline & M. phenylpyruvica, S. xylosus & $\begin{array}{l}\text { Ethylbenzene, benzaldehyde, benzonitrile, } \\
\text { 2-hydroxybenzaldehyde }\end{array}$ \\
\hline & $\begin{array}{l}\text { P. putida, E. coli, K. pneumoniae, } \\
\text { B. halodurans }\end{array}$ & Ethenylbenzene, ethylbenzene \\
\hline & Denitrifying bacteria & $\begin{array}{l}\text { 1-Phenylethanol, phenylethanone, } \\
\text { benzoyl-acetate }\end{array}$ \\
\hline Tryptophan & $\begin{array}{l}\text { Bacteroides, Lactobacillus, Clostridium, } \\
\text { Bifidodobacterium, Peptostreptococcus }\end{array}$ & $\begin{array}{l}\text { Indole, indoyl acetic acid and } \\
\text { indoyl propanoic acid }\end{array}$ \\
\hline \multirow[t]{2}{*}{ Cysteine } & Anaerobe & Elemental sulfur, hydrogen sulfide \\
\hline & & $\begin{array}{l}\text { Hydrogen sulfide, dimethyl sulfide, } \\
\text { dimethyl disulfide, dimethyl trisulfide, } \\
\text { dimethyl tetrasulfide }\end{array}$ \\
\hline \multirow[t]{3}{*}{ Methionine } & & $\begin{array}{l}\text { Methanethiol, dimethyl disulfide, } \\
\text { dimethyl trisulfide }\end{array}$ \\
\hline & Aerobe & Dimethyl sulfide \\
\hline & $\begin{array}{l}\text { H. alvei, E. agglomeran, S. liquefaciens, } \\
\text { A. putrefaciens and A. hydrophila }\end{array}$ & Methanethiol, dimethyl sulfide \\
\hline
\end{tabular}


formation of histamine and cadaverine. L-Arginine hydrolysis yields ornithine, which is then decarboxylated to putrescine. Decarboxylated lysine yields cadaverine (Gill-King 1999). The amino acids valine, leucine and isoleucine are fermented to higher alcohols such as propan-1-ol, 2-methyl-propan1-ol, 2-methyl-butan-1-ol and 3-methyl-butan-1-ol by the Ehrlich pathway or in reverse as by-products of anabolic biosynthetic pathways (Boumba et al. 2008; Chen 1978; Derrick and Large 1993). Moraxella phenylpyruvica, Staphylococcus xylosus and Staphylococcus starnosus transforme degrade leucine to 3-methyl-butan-1-ol, 3-methyl-butanal and 3-methylbutanoic acid (Moller et al. 1998; Stahnke 1999). 4-Methylphenol and propanoic acid phenylester are products of anaerobic tyrosine catabolism by Staphylococcus albus, Bacteroides fragilis, Fusobacterium sp., Bifidobacterium spp., Clostridium paraputrificum, C. butyricum, $C$. sporogenes and $C$. septicum. Tyrosine degradation also yields 2-phenylethanol, phenylacetaldehyde and phenylacetic acid (Smith and Macfarlane 1996). E. coli, Proteus sp., Enterococcus faecalis and $S$. albus produce phenol under facultative anaerobic conditions from the same amino acid (Bone et al. 1976). Phenylalanine degradation yields 1-phenylethanol, 2-phenylethanol, phenylacetaldehyde, phenylacetic acid and phenylpropanoic acid (Luengo et al. 2001; Macfarlane and Allison 1986; Rabus and Heider 1998; Smith and Macfarlane 1996). Tryptophan degradation by Bacteroides, Lactobacillus, Clostridium, Bifidobacterium and Peptostreptococcus yields indole, indoyl acetic acid and indoyl propanoic acid (Smith and Macfarlane 1996). L-Phenylalanine can form greenish products in the presence of phenylalanine deaminase and ferric ions $\left(\mathrm{Fe}^{3+}\right)$ (Gill-King 1999). Ferric ions are present in the soil and hemoglobin. Hemolytic Streptococcaceae can degrade hemoglobin, leading to the accumulation of greenish products in the dead vertebrate. Under aerobic conditions, indolic and phenolic compounds are oxidized by mono- and dioxygenases (Young and Rivera 1985). Under anaerobic conditions, these are fermented by methanogenic bacteria present in the large intestine to phenol, cyclohexanol, cyclohexanone, hexanoic acid, hexanedioic acid, butanoic acid, propanoic acid, acetic acid, $\mathrm{CO}_{2}, \mathrm{H}_{2}$ and methane (Young and Rivera 1985). Under aerobic conditions, Coccaceae, Pseudomonaceae, Bacteriaceae, Bacillaeceae, Candida sp, Saccharomyces $s p$ and certain higher fungi form pyruvate, acetyl-CoA and succinate. Possible byproducts are quinoline, glutamate, 2-butendioic acid, 2oxopropanoic acid and acetic acid (Evans 1963). Yeasts can degrade the amino acids threonine, leucine, isoleucine and valine to 1-propanol, 2-methyl-butan-1-ol, 3-methyl-butan-1ol and pentan-1-ol (Boumba et al. 2008).

Sulfur compounds are prominent VOCs emitted by decaying vertebrates (Stensmyr et al. 2002). The sulfur-containing amino acids cysteine and methionine play an important role in the formation of volatile sulfur compounds such as hydrogen sulfide, dimethyl sulfide, dimethyl disulfide, dimethyl trisulfide, dimethyl tetrasulfide and thiols (Dent et al. 2004; Freney 1967; Gill-King 1999; Kadota and Ishida 1972). The facultative anaerobic Proteus species from intestine, soil, and water releases hydrogen sulfide. Dimethyl sulfide is a product of methionine decomposition under aerobic conditions. Cysteine degradation can yield elemental sulfur under anaerobic conditions (Freney 1967). Methanethiol is a product of methionine degradation (Frederick et al. 1957; Kadota and Ishida 1972; Wolle et al. 2006), and it can lead to the formation of dimethyl disulfide during GC analysis (Lestremau et al. 2004). This oxidative dimerisation may also occur during the proteolysis of vertebrates (Frederick et al. 1957). Methanethiol and dimethyl sulfide (Lindinger et al. 1998) were found to be by-products of the metabolism of Hafnia alvei, Enterobacter agglomeran, Serratia liquefaciens, Alteromonas putrefaciens and Aeromonas hydrophila (Dainty et al. 1989). The amount of sulfur compounds emitted by bacteria is species-dependent (Stutz et al. 1991). Under aerobic conditions, some bacteria, such as those from the thiobacillus group, can transform sulfides to sulfurous acids, elemental sulfur, and sulfate (Dent et al. 2004). The formation of secondary products from sulfur compounds under natural and artificial conditions is an indicator of the reactivity of these compounds (Lestremau et al. 2004).

The variability of biogenic amines is high and depends on the micro-organisms growing on the tissue (Ansorena et al. 2002). Dimethylamine (Lindinger et al. 1998) or trimethylamine is formed by the oxidative decarboxylation of free amino acids by Sherwanella putrefaciens (Dainty et al. 1989; Lopez-Caballero et al. 2001). As these amines are not volatile under moderate temperature conditions, further degradation is necessary for the production of VOCs. In general, these compounds are further degraded to ammonia, oxidized to nitrite by Nitrosomonas spp. and further to nitrate by Nitrobacter spp. in the soil under aerobic conditions (Waksman and Starkey 1931). Aerobic Pseudomonaceae are proteolytic bacteria that use proline, glutamate, lysine or Lphenylalanine as their $\mathrm{C}$ and $\mathrm{N}$ sources (Herrera et al. 2010).

Facultative or obligate aerobic Bacillaceae excrete the non-specific proteolytic enzyme subtilisin (Higgins and Burns 1975). Micrococcaceae such as Micrococcus luteus are aerobic and abundant in the soil and surface water and are also present on the skin of vertebrates. The proteolytic species Serratia proteamaculans, Pseudomonas fragi and Carnobacterium maltaromaticum were detected on beef (Ercolini et al. 2009). Other micro-organisms reported to grow on muscle tissue are the facultative anaerobe Brochotrix thermosphacta and the facultative anaerobe and aero-tolerant Leuconostoc mesenteroides, Leuc. curvatus, Pseudomonas lundensis and Pseudomonas fluorescens biovar. Moraxella species were also identified (Dainty et al. 1985; Stutz et al. 1991; Vanderzant et al. 1986). These 
species are generally abundant in the respiratory tract (Molin and Ternstrom 1986).

\section{Lipids}

In vertebrates, all cells contain fat in their phospholipid membranes. Fatty acids such as linoleic acid or arachidonic acid are typically present in abundance in lipid membranes (Frankel 1983). Intramuscular fat consists of fat cells inside the muscular tissue (Gandemer 2002), and depot fat is the fat under the skin. Intramuscular fat and depot fat contain triglycerides. The hydrolysis of triglycerides by microbial lipolytic enzymes yields glycerol and fatty acids.

Glycerol degradation results in the formation of pyruvate, ATP and NADH. Pyruvate degradation yields products such as butan-1-ol, butanoic acid, acetone, ethanol, propionic acid, acetic acid, formic acid, lactic acid, propane-1,3-diol, propane-1,2-diol and butane-2,3-diol (Boumba et al. 2008). Many Clostridiaceae are obligate anaerobic species that ferment pyruvate to acetone, ethanol, butan-1-ol, acetic acid, butanoic acid or butane-1,3-diol. E. coli catabolizes glycerol to propanol and propanoic acid (Boumba et al. 2008).

The fatty acids of vertebrates are further oxidized or hydrogenised (Dent et al. 2004) to several volatile compounds. However, there is a lack of detailed studies on volatile formation by microbial fatty acid degradation (Combet et al. 2006; Kionka and Kunau 1985). Although the beta-oxidation enzymes of prokaryotes, fungi, and plants differ from each other (Heath et al. 2002; Kionka and Kunau 1985), their catabolic activity is comparable and yields acetyl-CoA or propionyl-CoA (Gottschalk 1986). Acetyl$\mathrm{CoA}$ is degraded to either ethanol, acetic acid, acetaldehyde, and acetone or to propan-1-ol, propan-2-ol, and propionic acid (Boumba et al. 2008). The volatile by-products of lipoxygenase activity in fungi are mainly C6 or C9 aldehydes and $\mathrm{C} 8$ alcohols or $\mathrm{C} 8$ ketones (Combet et al. 2006; Feussner et al. 1997).

Aerobic oxidation of linoleic acid yields 2,4-decadienal, 3nonenal, hexanal, 2,4,7-decatrienal, 2,6-nonadienal, 3-hexenal, and propanal. Further chemical groups include ketones, hydrocarbons, acids, and epoxides. C. perfringens (welchii) (Polson et al. 1985) or other species from the genera Pseudomonas, Acinetobacter, and Bacillus (Gottschalk 1986) are lipolytic and yield volatile aldehydes, ketones, or esters (Boumba et al. 2008; Dent et al. 2004). Oxidation of linoleic acid in fungi yields the eight-carbon volatiles 1-octen3-ol, octan-3-ol, octan-1-ol, 1-octen-3-one, and octan-3-one (Combet et al. 2006). Tetradecanoic acid, hexadecanoic acid, octadecanoic acid, 9-octadecenoic acid, 10hydroxyoctadecanoic acid, and $(Z, Z)-9,12$-octadecadienoic acid are part of the adipocere. The latter can form in the later stages of decay by saponification of fatty acids under anaerobic conditions, neutral or slightly alkaline $\mathrm{pH}$, and in the presence of certain enzymes (Forbes et al. 2002, 2005a, b, c; Gill-King 1999; Notter et al. 2009).

Bones

Bones consist of organic and mineral components. The organic components are mainly fat and proteins. In ancient bones, it is possible to detect triglycerides, free fatty acids, collagen, amino acids, and non-collagenous proteins such as osteocalcin and haemoglobins (Evershed et al. 1995; Schultz 2006; Vanklinken et al. 1994; Vanklinken and Hedges 1992). Therefore, fat degradation can lead to the release of volatiles in the later stages of decay. Fat is the major component of marrow consisting of different saturated and unsaturated fatty acids, omega- 6 polyunsaturated fatty acid (PUFA), and omega-3 PUFA (Yeung et al. 2008). Vass et al. investigated the volatile emissions of exposed mammalian bones. They found different ratios of ketones, aldehydes, and alcohols in the four different mammalian species, and among other compounds they identified nonan-2-one, nonanal, hexanal, and hexan-1-ol (Vass et al. 2008). Ketones and aldehydes are products of fat degradation (Boumba et al. 2008; Dent et al. 2004). Alcohols, especially hexan-1-ol and 1-octen-3-ol, are by-products of fungal metabolism (Borjesson et al. 1993; Korpi et al. 1998; Larsen and Frisvad 1995; Pasanen et al. 1996; Sunesson et al. 1996; Thakeow et al. 2008), and signs of fungal activity are present on bones (Marinho et al. 2006). ROS might also play a role in the formation of volatiles from bones (Choe and Min 2005). Of the total bone proteins, $90 \%$ to $95 \%$ consist of different collagen proteins. These are degraded to peptides by bacterial collagenases (Macfarlane and Allison 1986). Streptomyces spp. are aerobic bacteria living in the soil, and they can cleave the strong disulfide bonds in the collagen molecule (Gray and Williams 1971). The mineral component of bones consists of hydroxyapatite, which is degraded by physical weathering.

\section{Microbial interactions}

Micro-organisms can show ecological interactions such as mutualism or competition. These interactions affect the pattern of volatiles released by the decaying vertebrate. Smith et al. investigated the formation of phenolic and indolic compounds by intestinal microbes in feces under anaerobic conditions and found variations in the relative quantity of phenolic and indolic volatiles, depending on the microbial density (Smith and Macfarlane 1996). Therefore, microbial density seems to influence their metabolic pathways due to inter- and intraspecific interactions. An example of mutualism is the fermentation of ethanol to acetate and $\mathrm{H}_{2}$. As the $\mathrm{H}_{2}$ concentration increases, the growth of the ethanol- 
fermenting microbes decreases. The presence of microbes that utilize $\mathrm{H}_{2}$ to reduce $\mathrm{CO}_{2}$ to methane increases the growth rate of the first micro-organism (Bryant et al. 1967). The suppression of microbial ethanol fermentation in the presence of hydrogen-utilizing micro-organisms is an example of the competition between microbes (Iannotti et al. 1973). The lower partial pressure of $\mathrm{H}_{2}$ leads to its formation from NADH by ethanol-fermenting microbes instead of coupling of the oxidation of NADH to the reduction of acetyl-CoA to ethanol. Both mechanisms may be important for post-mortem ethanol detection. The mycelial growth and spore germination of fungi are inhibited by $P$. fluorescens and other Pseudomonaceae (Fernando et al. 2005). Dimethyl trisulfide is one of the inhibitory substances utilized by the bacteria in this competitive interaction. Phenol is an antiseptic substance emitted by proteolytic Bacillaceae $(\mathrm{Gu}$ et al. 2007), E. coli, Proteus sp., Enterococcus faecalis and S. albus (Bone et al. 1976), and it might constrain the growth of other microbes. Trichosporon cutaneum can degrade phenol, but the presence of cresols such as 4-methylphenol delays this reaction (Alexieva et al. 2008). 4-Methylphenol is a metabolic product of $S$. albus, B. fragilis, Fusobacterium sp., Bifidobacterium spp., C. paraputrificum, C. butyricum, $C$. sporogenes, and C. septicum. All these data suggest a complex interaction based on phenolic compounds. The lipid metabolism of microbes yields fatty acids that show an antimicrobial effect (Jay 2000). In particular, the emission of aldehydes increases during the later stages of decay (Dekeirsschieter et al. 2009; Vass et al. 2008). This might lead to the conclusion that fatty acids are prominently decomposed in the later stages of decay to form aldehydes and that interactions based on fatty acids might occur during these stages. Volatiles emitted by the environment can also influence microbial growth on decaying vertebrates (Gilbert et al. 1969). For instance, thiols and sulfides were found to stimulate (King and Coley-Smith 1969) or suppress fungal growth (Lewis and Papavizas 1972). These results show the complexity of microbial interactions, which affect the VOCs emitted.

\section{Post-mortem volatiles}

The results of volatile analysis are influenced by abiotic factors, sampling technique, and analytical setup. Abiotic factors directly influence the release of volatiles from a vertebrate. As mentioned above, the temperature influences the activity of volatile-generating enzymes and the microbial species composition. Moisture also affects the process.

The compounds identified by volatile analysis depend on the sampling technique and the analytical setup. The sampling technique can be passive, for instance, in a closed container, or active with a constant gas flow. Depending on the exposed adsorbent, the compounds enrich according to their chemical properties. A non-specific material adsorbs many different volatiles, whereas a specific material is selective for characteristics such as polarity or certain functional groups. As an example, the SPME (Solid Phase Micro Extraction) technique was developed for uncomplicated and fast volatile sampling in a static headspace. A short fiber covered with an adsorption material is exposed to the headspace of the sample. After equilibration between the solid and the gaseous phase, the fiber is directly inserted in the injector of a GC system and the volatiles are thermally desorbed for analysis. The surface of the SPME fibers is modified to adsorb different classes of volatiles. For instance, polydimethylsiloxane (PDMS)-covered fibers adsorb predominantly non-polar compounds or Carbowaxcovered fibers adsorb predominantly polar compounds. There exist several surface adsorbents, which can be combined with each other in order to achieve selectivity or non-selectivity during sampling. Some fiber types used in analytical studies are listed in Table 4. The exposure time of the fiber is important as well because a short exposure time favors the adsorption of compounds with a high volatility, while discriminating compounds with a low volatility. A long exposure will lead to desorption of highly volatile compounds, while additional low-volatility compounds are adsorbed. Moreover, individual adsorption properties may vary between fibers from different lots. Therefore, quantification with the SPME technique requires an internal standard with known quantity. SPME can be used for the extraction of compounds from liquid solutions (Ercolini et al. 2009). The liquid extracts can be esterified to enhance the trace analysis (Forbes et al. 2002, 2005a, b, c). For more information on SPME sampling, see Tholl et al. (2006). Dynamic headspace sampling techniques are used to find volatiles with low emission rates. A continuous air stream is led through an adsorbent to trap the volatiles out of the sampling system. Either the air is circulated in the sampling system or filtered air enters the system, is enriched with volatiles from the sample, and led through the adsorbent and then out of the system. For dynamic headspace sampling, different adsorbents can be used. Tubes filled with different selective or unselective adsorbent powders, for instance, TENAX, Carbotrap, Carbotrap C, and Carbosieve S-III, are commercially available. For increasing performance, a tube can be filled with different adsorbent powders. Vass et al. used triple-sorbent traps for their analysis of buried human remains (Vass et al. 2004, 2008). Another possibility is the trapping of the volatiles by directing the gas flow through cooled liquid solvents such as cyclohexane, methanol, or dichloromethane. Tholl et al. (2006) give a more detailed description of volatile sampling techniques (Tholl et al. 2006).

Any chemical analysis of the adsorbed volatile compounds has its own analytical window that enables the 
identification of certain volatiles while excluding others. For instance, the polarity of a compound influences its retention on a GC column. A low molecular weight polar compound might only be detectable by high retention on a polar column and might be undetectable because of lower retention on a non-polar column. Furthermore, the detection of stereo isomers requires enantiomer-selective columns with modified surfaces (Dotterl et al. 2006). The identification of isotope ratios, like ${ }^{13} \mathrm{C} /{ }^{12} \mathrm{C}$ or ${ }^{15} \mathrm{~N} /{ }^{14} \mathrm{~N}$, requires a modified GC setup (Boschker and Middelburg 2002; Meier-Augenstein 2002). Both techniques can be used to specify trace analytical results. Especially, the identification of isotope ratios in cadaveric volatile compounds can give important information on the nutrition of the dead vertebrate or the microbial communities growing on it (Boschker and Middelburg 2002; Meier-Augenstein 2002).

Finally, the detector attached to the column determines the information collected on the substances. A flame ionization detector (FID) gives information about the retention time and the peak area of compounds in a sample. Identification and quantification can only be achieved by using standard compound samples with known concentration. However, the selection of standard compounds is problematic when the compound to be identified is unknown. A mass spectrometer (MS) is able to identify compounds not only by the retention time of standard compounds but also by comparing the fragment pattern of a compound in a sample with a fragment pattern database. However, a standard compound sample has to be used for exact identification as well because the fragment pattern database is restricted to some thousand compounds and several compounds have the same fragment pattern. For quantification, a reference quantity is necessary as well. The selection of possible standard compounds is much easier with a MS system than with a FID because the database gives a pre-selection of possible candidates. However, a false identification is still possible when two compounds have very similar mass spectra and the same retention time. In order to improve the trace analysis, it is possible to use two-dimensional gas chromatography ( $\mathrm{GC} \times$ GC). Two columns with different polarity are coupled to each other and, as a result, every compound has two retention indices. Two compounds, which have the same retention time on a non-polar column, might have a different retention time on the second polar column in a two-dimensional GC. Another approach to enhance compound identification is the time of flight MS (TOFMS). This detector measures the fragment-specific flight time from the point of fragmentation to the detector plate. TOFMS analysis allows a more precise differentiation between atomic masses in comparison to conventional mass spectrometers. The studies listed in Table 4 use GC/ MS analysis; only Kalinova et al. is an exception due to using GC $\times$ GC-TOFMS (Kalinova et al. 2009). Hübschmann et al. give further information on gas chromatography and coupled detector systems (Hübschmann 2009). Since it is known that the analytical method influences the results, Table 4 has been provided to supplement Table 3 and list selected information on sampling, sample preparation, and sample analysis. Another factor in chemical analysis is the modification of volatile compounds during the analytical procedure. For instance, volatile sulfur compounds can be transformed during GC-MS analysis (Lestremau et al. 2004).

The analysis of post-mortem volatiles does not only depend on the analytical parameters itself. Other decomposers might influence the volatile pattern of a decaying vertebrate by feeding. Scratches or holes made by scavengers can open the dead body before it breaks up due to swelling. As a result, oxygen will infiltrate the body, favoring the growth of aerobic or facultative aerobic microbial species and suppressing that of obligate anaerobes. Aerobic (Straehlerpohl and Exner 1985) and anaerobic (Gulzow 1982) micro-organisms of the scavengers oral cavities could be introduced. Larval growth might influence the volatile pattern because proteolytic enzymes are excreted by the larvae. The cuticle hydrocarbons of these insects might also contribute to the volatile pattern. Burying beetles (Nicrophorus spp.) even bury small vertebrates and inhibit the decay with excretions from their glands (Kalinova et al. 2009).

Table 3 lists the VOCs reported in studies on the decay of vertebrate tissue. Because of the large number of reported VOCs, the list contains only those that have been identified in more than one study. The volatile compounds butane-1,2-diol, butane-1,3-diol, propane-1,3-diol, propane1,2-diol, butane-2,3-diol, propan-1-ol, and propan-2-ol are not reported in any of the reviewed experimental studies although they are known to be volatile products of amino acid degradation (Boumba et al. 2008). Table 4 contains additional information about the method used.

\section{Application of post-mortem volatiles}

Insects are important cues for criminal investigations as their larval development on corpses can help to estimate the time since death. It is not possible to determine the exact day of death as larval development is influenced by many environmental factors. Therefore, additional cues are necessary to support juristically accepted estimation of the pmi. Several authors suggested the use of insects to select volatiles, which are important in the dynamic emission pattern of vertebrate decay (LeBlanc and Logan 2010; Paczkowski et al. 2011; Statheropoulos 2005; Vass et al. 2002). These volatiles could be an additional cue for determining the time since death. However, the variation of cadaveric volatiles is very high. In order to find useful 
Table 3 VOCs identified in experimental studies and reviews. The cross-references to Table 4 contain information on the experimental conditions

\begin{tabular}{llll}
\hline Compound & $\begin{array}{l}\text { CAS } \\
\text { number }\end{array}$ & Origin & Publication \\
\end{tabular}

Alcohols

Ethanol

$64-17-5$

2-Methyl-propan-1-ol

$78-83-1$

Butan-1-ol

Butan-2-ol

2-Methyl-butan-1-ol

3-Methyl-butan-1-ol

Pentan-1-ol

Hexan-1-ol

2-Ethyl-hexan-1-ol

Octan-1-ol

1-Octen-3-ol

Acids

Formic acid

Acetic acid

Propanoic acid
71-41-0

111-87-5

3391-86-4

64-18-6

64-19-7

78-92-2

$137-32-6$

$123-51-3$

$111-27-3$

104-76-7

Fungal metabolism

(Borjesson et al. 1993;

Korpi et al. 1998;

Larsen and Frisvad 1995;

Pasanen et al. 1996;

Sunesson et al. 1996;

Thakeow et al. 2008)

Carbohydrates
(Boumba et al. 2008)

L-Leucine (Moller et al. 1998)

Threonine, leucine, isoleucine, valine (Boumba et al. 2008)

Linoleic acid

(Combet et al. 2006)

Linoleic acid

(Combet et al. 2006)

Glycerol, pyruvate

(Boumba et al. 2008)

Carbohydrates, indolic/phenolic compounds, glycerol (Boumba et al. 2008;

Evans 1963; Smith and

Macfarlane 1996;

Young and Rivera 1985)

Amino acids

(Demeyer and Vannevel 1979)
(Gottschalk 1986; Corry 1978; O`Neal and Poklis 1996; Stutz et al. 1991;

Dent et al. 2004; Statheropoulos et al. 2005;

Statheropoulos et al. 2007;

Boumba et al. 2008;

Dekeirsschieter et al. 2009) $2,6,7,17$

(Derrick and Large 1993;

O'Neal and Poklis 1996;

Statheropoulos et al. 2005;

Boumba et al. 2008;

Dekeirsschieter et al. 2009) $)^{3,6}$

(O'Neal and Poklis 1996; Gill-King 1999;

Dent et al. 2004; Statheropoulos et al. 2005;

Boumba et al. 2008;

Dekeirsschieter et al. 2009) $)^{1,2,3,6}$

(O'Neal and Poklis 1996;

Dekeirsschieter et al. 2009) $)^{1,2,3}$

(Derrick and Large 1993; Boumba et al. 2008)

(Derrick and Large 1993; O`Neal and

Poklis 1996; Boumba et al. 2008;

Dekeirsschieter et al. 2009;

Ercolini et al. 2009; $)^{1,2,3,18}$

(Lorenzo et al. 2003; Statheropoulos et al. 2005; Vass et al. 2008; Boumba et al. 2008;

Dekeirsschieter et al. 2009;

Hoffman et al. 2009) ${ }^{1,2,3,5,6,8}$

(Stutz et al. 1991; Haze et al. 2001;

Statheropoulos et al. 2005;

Hoffman et al. 2009) ${ }^{6,8,9,17}$

(Vass et al. 2004; Statheropoulos et al. 2007;

Dekeirsschieter et al. 2009;

Ercolini et al. 2009;

Hoffman et al. 2009)

(Haze et al. 2001; Combet et al. 2006; Hoffman et al. 2009) $)^{8,9}$

(Zhang et al. 1992; Combet et al. 2006;

Ercolini et al. 2009;

Hoffman et al. 2009) ${ }^{8,18,19}$

(Gottschalk 1986;

Dekeirsschieter et al. 2009) $)^{1,2}$

(Gottschalk 1986; Dent et al. 2004;

Boumba et al. 2008)

(Gill-King 1999; Statheropoulos et al. 2005;

Boumba et al. 2008;

Dekeirsschieter et al. 2009;

Hoffman et al. 2009; $)^{1,2,3,6,8}$ 
Table 3 (continued)

\begin{tabular}{|c|c|c|c|}
\hline Compound & $\begin{array}{l}\text { CAS } \\
\text { number }\end{array}$ & Origin & Publication \\
\hline Butanoic acid & $107-92-6$ & $\begin{array}{l}\text { Carbohydrates, lipids } \\
\text { (Boumba et al. 2008), } \\
\text { amino acids } \\
\text { (Demeyer and Vannevel 1979) }\end{array}$ & $\begin{array}{l}\text { (Gill-King 1999; Lorenzo et al. 2003; } \\
\text { Dent et al. 2004; Statheropoulos et al. 2005; } \\
\text { Boumba et al. 2008; Dekeirsschieter et al. 2009; } \\
\text { Hoffman et al. 2009) } \text { ) }^{1,2,3,6,8}\end{array}$ \\
\hline Pentanoic acid & $109-52-4$ & $\begin{array}{l}\text { Amino acids } \\
\text { (Demeyer and Vannevel 1979) }\end{array}$ & $\begin{array}{l}\text { (Lorenzo et al. 2003; Dekeirsschieter et al. 2009; } \\
\text { Hoffman et al. 2009; ) } \text { ) }^{1,2,3,8}\end{array}$ \\
\hline Hexanoic acid & $142-62-1$ & $\begin{array}{l}\text { Indolic/phenolic compounds } \\
\text { (Young and Rivera 1985) }\end{array}$ & $\begin{array}{l}\text { (Lorenzo et al. 2003; Dekeirsschieter et al. 2009; } \\
\text { Hoffman et al. 2009) }\end{array}$ \\
\hline Tetradecanoic acid & $544-63-8$ & Lipids (Gill-King 1999) & $\begin{array}{l}\text { (Forbes et al. 2002, 2005a, b, c; } \\
\text { Notter et al. 2009) }\end{array}$ \\
\hline Hexadecanoic acid & $57-10-3$ & Lipids (Gill-King 1999) & $\begin{array}{l}\text { (Forbes et al. 2002, 2005a, b, c; } \\
\text { Notter et al. 2009) }\end{array}$ \\
\hline Octadecanoic acid & $57-11-4$ & Lipids (Gill-King 1999) & $\begin{array}{l}\text { (Forbes et al. 2002, 2005a, b, c; } \\
\text { Notter et al. 2009) }\end{array}$ \\
\hline 9-Octadecenoic acid & $112-80-1$ & Lipids (Gill-King 1999) & $\begin{array}{l}\text { (Yan et al. 2001; Forbes et al. 2002, 2005a, b, c; } \\
\text { Notter et al. 2009) }\end{array}$ \\
\hline$(Z, Z)-9,12$-Octadecadienoic acid & $60-33-3$ & Lipids (Gill-King 1999) & (Nushida et al. 2008; Notter et al. 2009) $^{15,16}$ \\
\hline 10-Hydroxyoctadecanoic acid & $638-26-6$ & Lipids (Gill-King 1999) & $\begin{array}{l}\text { (Forbes et al. 2002; } \\
\text { Forbes et al. 2005a, b, c) }{ }^{11,12,13,14}\end{array}$ \\
\hline \multicolumn{4}{|l|}{ Esters } \\
\hline Acetic acid propyl ester & $109-60-4$ & & $\begin{array}{l}\text { (Statheropoulos et al. 2005; } \\
\text { Dekeirsschieter et al. 2009) }\end{array}$ \\
\hline Butanoic acid ethyl ester & $105-54-4$ & & (Statheropoulos et al. 2005; Hoffman et al. 2009) $^{6,8}$ \\
\hline Butanoic acid butyl ester & $109-21-7$ & & $\begin{array}{l}\text { (Lorenzo et al. 2003; Dekeirsschieter et al. 2009; } \\
\text { Hoffman et al. 2009; ) } \text { ) }^{1,2,3,8}\end{array}$ \\
\hline \multicolumn{4}{|l|}{ Aldehydes } \\
\hline Butanal & $123-72-8$ & Alkanes (Arp 1999) & $\begin{array}{l}\text { (Stutz et al. 1991; Vass et al. 2008; } \\
\text { Dekeirsschieter et al. 2009) }\end{array}$ \\
\hline Pentanal & $110-62-3$ & Lipids (Boumba et al. 2008) & $\begin{array}{l}\text { (Statheropoulos et al. 2005; Vass et al. 2008; } \\
\text { Dekeirsschieter et al. 2009) }\end{array}$ \\
\hline Hexanal & $66-25-1$ & Lipids (Boumba et al. 2008) & $\begin{array}{l}\text { (Haze et al. 2001; Lorenzo et al. 2003; } \\
\text { Statheropoulos et al. 2005; Hoffman et al. 2009; } \\
\text { Ercolini et al. 2009) }\end{array}$ \\
\hline Heptanal & $111-71-7$ & Lipids (Boumba et al. 2008) & $\begin{array}{l}\text { (Stutz et al. 1991; Haze et al. 2001; } \\
\text { Lorenzo et al. 2003; Vass et al. 2008; } \\
\text { Dekeirsschieter et al. 2009; } \\
\text { Hoffman et al. 2009) }{ }^{1,2,5,8,9,17}\end{array}$ \\
\hline Nonanal & $124-19-6$ & Lipids (Boumba et al. 2008) & $\begin{array}{l}\text { (Haze et al. 2001; Vass et al. 2004; } \\
\text { Vass et al. 2008; Ercolini et al. 2009; } \\
\text { Hoffman et al. 2009) }\end{array}$ \\
\hline Decanal & $112-31-2$ & & (Vass et al. 2004; Vass et al. 2008) $^{4,5}$ \\
\hline \multicolumn{4}{|l|}{ Ketones } \\
\hline Acetone & $67-64-1$ & $\begin{array}{l}\text { Carbohydrates } \\
\text { (Boumba et al. 2008) }\end{array}$ & $\begin{array}{l}\text { (Stutz et al. 1991; O`Neal and Poklis 1996; } \\
\text { Gill-King 1999; Vass et al. 2004; } \\
\text { Dent et al. 2004; Statheropoulos et al. 2005; } \\
\text { Statheropoulos et al. 2007; Boumba et al. 2008; } \\
\text { Dekeirsschieter et al. 2009) }\end{array}$ \\
\hline Butan-2-one & $78-93-3$ & Alkanes (Patel et al. 1979) & $\begin{array}{l}\text { (Stutz et al. 1991; Statheropoulos et al. 2005; } \\
\text { Statheropoulos et al. 2007; } \\
\text { Dekeirsschieter et al. 2009) }\end{array}$ \\
\hline Pentan-2-one & $107-87-9$ & Alkanes (Patel et al. 1979) & $\begin{array}{l}\text { (Statheropoulos et al. 2005; } \\
\text { Dekeirsschieter et al. 2009) }\end{array}$ \\
\hline Cyclohexanone & $108-94-1$ & $\begin{array}{l}\text { Indolic/phenolic compounds } \\
\text { (Young and Rivera 1985) }\end{array}$ & $\begin{array}{l}\text { (Statheropoulos et al. } 2005 \\
\text { Hoffman et al. 2009) }\end{array}$ \\
\hline
\end{tabular}


Table 3 (continued)

\begin{tabular}{|c|c|c|c|}
\hline Compound & $\begin{array}{l}\text { CAS } \\
\text { number }\end{array}$ & Origin & Publication \\
\hline Heptan-2-one & $110-43-0$ & & $\begin{array}{l}\text { (Statheropoulos et al. 2005; } \\
\text { Dekeirsschieter et al. 2009; } \\
\text { Hoffman et al. 2009) }\end{array}$ \\
\hline Nonan-2-one & $821-55-6$ & $\begin{array}{l}\text { Lipids } \\
\text { (Boumba et al. 2008) }\end{array}$ & $\begin{array}{l}\text { (Statheropoulos et al. 2007; Vass et al. 2008; } \\
\text { Dekeirsschieter et al. 2009; } \\
\text { Ercolini et al. 2009) }\end{array}$ \\
\hline \multicolumn{4}{|l|}{ Homoaromatic compounds } \\
\hline Benzene & $71-43-2$ & & (Statheropoulos et al. 2005; Vass et al. 2008) \\
\hline Methylbenzene & $108-88-3$ & & $\begin{array}{l}\text { (Stutz et al. 1991; Vass et al. 2004; } \\
\text { Statheropoulos et al. 2005; } \\
\text { Statheropoulos et al. 2007; } \\
\text { Vass et al. 2008; Hoffman et al. 2009) })^{4,5,6,7,8,17}\end{array}$ \\
\hline 1,2-Dimethylbenzene & $95-47-6$ & & (Statheropoulos et al. 2007; Vass et al. 2008) \\
\hline 1,3-Dimethylbenzene & $108-38-3$ & & $\begin{array}{l}\text { (Statheropoulos et al. 2005; } \\
\text { Statheropoulos et al. 2007) }\end{array}$ \\
\hline 1,4 Dimethylbenzene & $106-42-3$ & & $\begin{array}{l}\text { (Vass et al. 2004; Statheropoulos et al. 2005; } \\
\text { Statheropoulos et al. 2007; Vass et al. 2008; } \\
\text { Hoffman et al. 2009) }\end{array}$ \\
\hline 1,2,3-Trimethylbenzene & $526-73-8$ & & $\begin{array}{l}\text { (Statheropoulos et al. 2005; } \\
\text { Statheropoulos et al. 2007) }\end{array}$ \\
\hline Ethylbenzene & $100-41-4$ & $\begin{array}{l}\text { Phenylalanine } \\
\text { (Luengo et al. 2001; } \\
\text { Moller et al. 1998) }\end{array}$ & $\begin{array}{l}\text { (Vass et al. 2004; Statheropoulos et al. 2005; } \\
\text { Vass et al. 2008) }\end{array}$ \\
\hline Ethenylbenzene & $100-42-5$ & $\begin{array}{l}\text { Phenylalanine } \\
\text { (Luengo et al. 2001) }\end{array}$ & $\begin{array}{l}\text { (Vass et al. 2004; Statheropoulos et al. 2007; } \\
\text { Vass et al. 2008) }\end{array}$ \\
\hline 1-Methyl-2-ethylbenzene & $611-14-3$ & & $\begin{array}{l}\text { (Vass et al. 2004; Statheropoulos et al. 2005; } \\
\text { Vass et al. 2008) }\end{array}$ \\
\hline 2-Phenyl-propan-2-ol & $617-94-7$ & & (Vass et al. 2004; Vass et al. 2008) \\
\hline Phenol & $108-95-2$ & $\begin{array}{l}\text { Tyrosine } \\
\text { (Bone et al. 1976) }\end{array}$ & $\begin{array}{l}\text { (Cummings et al. 1979; } \\
\text { Statheropoulos et al. 2007; } \\
\text { Vass et al. 2008; } \\
\text { Dekeirsschieter et al. 2009) })^{1,2,3,5,7,8}\end{array}$ \\
\hline 4-Methylphenol & $106-44-5$ & $\begin{array}{l}\text { Tyrosine } \\
\text { (Bone et al. 1976) }\end{array}$ & $\begin{array}{l}\text { (Geypens et al. 1997; } \\
\text { Statheropoulos et al. 2007; } \\
\text { Dekeirsschieter et al. 2009) }\end{array}$ \\
\hline 2-Phenylethanol & $60-12-8$ & $\begin{array}{l}\text { Phenylalanine } \\
\text { (Luengo et al. 2001) }\end{array}$ & $\begin{array}{l}\text { (O`Neal and Poklis 1996; } \\
\text { Dekeirsschieter et al. 2009) }\end{array}$ \\
\hline Benzaldehyde & $100-52-7$ & $\begin{array}{l}\text { Phenylalanine } \\
\text { (Moller et al. 1998) }\end{array}$ & $\begin{array}{l}\text { (Stutz et al. 1991; Lorenzo et al. 2003; } \\
\text { Vass et al. 2004; Dekeirsschieter et al. 2009; } \\
\text { Hoffman et al. 2009) }\end{array}$ \\
\hline Acetophenone & $98-86-2$ & $\begin{array}{l}\text { Phenylalanine } \\
\text { (Rabus and Heider 1998) }\end{array}$ & $\begin{array}{l}\text { (Statheropoulos et al. 2007; } \\
\text { Dekeirsschieter et al. 2009) }\end{array}$ \\
\hline 1-Methoxy-propylbenzene & $59588-12-4$ & & (Vass et al. 2004; Vass et al. 2008) \\
\hline Naphthalene & $91-20-3$ & & $\begin{array}{l}\text { (Vass et al. 2004; Statheropoulos et al. 2007; } \\
\text { Vass et al. 2008) }\end{array}$ \\
\hline \multicolumn{4}{|l|}{ Nitrogen compounds } \\
\hline Trimethylamine & $75-50-3$ & $\begin{array}{l}\text { Amino acids } \\
\text { (Lopez-Caballero et al. 2001) }\end{array}$ & $\begin{array}{l}\text { (Dainty et al. 1989; Stutz et al. 1991; } \\
\text { Dekeirsschieter et al. 2009) }\end{array}$ \\
\hline Benzonitrile & $100-47-0$ & $\begin{array}{l}\text { L-Phenylalanine } \\
\text { (Moller et al. 1998) }\end{array}$ & (Vass et al. 2004; Dekeirsschieter et al. 2009) $^{1,2,4}$ \\
\hline Indole & $120-72-9$ & $\begin{array}{l}\text { Tryptophan } \\
\text { (Smith and Macfarlane 1996) }\end{array}$ & $\begin{array}{l}\text { (O'Neal and Poklis 1996; Gill-King 1999; } \\
\text { Vass et al. 2002; Lorenzo et al. 2003; } \\
\text { Dent et al. 2004; Statheropoulos et al. 2005; } \\
\text { Hoffman et al. 2009; } \\
\text { Dekeirsschieter et al. 2009) }\end{array}$ \\
\hline
\end{tabular}


Table 3 (continued)

\begin{tabular}{|c|c|c|c|}
\hline Compound & $\begin{array}{l}\text { CAS } \\
\text { number }\end{array}$ & Origin & Publication \\
\hline \multicolumn{4}{|l|}{ Sulfur compounds } \\
\hline Sulfur dioxide & $7446-09-5$ & & $\begin{array}{l}\text { (Vass et al. 2002; Vass et al. 2004; } \\
\text { Statheropoulos et al. 2005; Vass et al. 2008; } \\
\text { Dekeirsschieter et al. 2009) }\end{array}$ \\
\hline Carbon disulfide & $75-15-0$ & & $\begin{array}{l}\text { (Vass et al. 2004; Statheropoulos et al. 2005; } \\
\text { Vass et al. 2008) }\end{array}$ \\
\hline Methanethiol & $74-93-1$ & $\begin{array}{l}\text { Methionine } \\
\text { (Frederick et al. 1957; } \\
\text { Kadota and Ishida 1972) }\end{array}$ & $\begin{array}{l}\text { (Lindinger et al. 1998; Dekeirsschieter et al. 2009; } \\
\text { Kalinova et al. 2009) }\end{array}$ \\
\hline Dimethyl sulfide & $75-18-3$ & $\begin{array}{l}\text { Methionine } \\
\text { (Dainty et al. 1989) }\end{array}$ & $\begin{array}{l}\text { (Stutz et al. 1991; Lindinger et al. 1998; } \\
\text { Vass et al. 2004; Statheropoulos et al. 2005; } \\
\text { Statheropoulos et al. 2007; Vass et al. 2008; } \\
\text { Kalinova et al. 2009) }\end{array}$ \\
\hline Dimethyl disulfide & $624-92-0$ & $\begin{array}{l}\text { Methanethiol } \\
\text { (Frederick et al. 1957; } \\
\text { Lestremau et al. 2004) }\end{array}$ & $\begin{array}{l}\text { (Stutz et al. 1991; Stensmyr et al. 2002; } \\
\text { Lorenzo et al. 2003; Dent et al. 2004; } \\
\text { Vass et al. 2004; Statheropoulos et al. 2005; } \\
\text { Statheropoulos et al. 2007; Vass et al. 2008; } \\
\text { Dekeirsschieter et al. 2009; Hoffman et al. 2009; } \\
\text { Kalinova et al. 2009) }\end{array}$ \\
\hline Dimethyl trisulfide & $3658-80-8$ & $\begin{array}{l}\text { Methionine } \\
\text { (Wolle et al. 2006) }\end{array}$ & $\begin{array}{l}\text { (Stutz et al. 1991; Stensmyr et al. 2002; } \\
\text { Dent et al. 2004; Vass et al. 2004; } \\
\text { Statheropoulos et al. 2005; } \\
\text { Statheropoulos et al. 2007; Vass et al. 2008; } \\
\text { Dekeirsschieter et al. 2009; } \\
\text { Kalinova et al. 2009) }\end{array}$ \\
\hline
\end{tabular}

marker volatiles, which are correlated to the time since death, it is possible to use insects as an information filter. Many insects have adapted their olfaction over millions of years to specific cadaveric volatiles (Huotari and Mela 1996; Kaib 1974; Kalinova et al. 2009; Stensmyr et al. 2002). Insects are feasible organisms for olfactory research because electrophysiological methods have been established for many species (Kalinova et al. 2009; Schutz et al. 1999; Stensmyr et al. 2002; Thakeow et al. 2008; Weissbecker et al. 2004), and the molecular basis of insect olfaction has been investigated for decades (Vogt and Riddiford 1981; Wicher et al. 2008). The order Diptera, in which most necrophilic insect species are classified, is relatively easy to breed at a high reproduction rate. Using chemo-ecological methods that combine trace analysis and electrophysiology (Weissbecker et al. 2004), these insects can be used to identify core compounds from the volatile pattern of a dead vertebrate. The existence of a common core of decompositional odors has already been suggested (Dekeirsschieter et al. 2009). Carrion-visiting Diptera can be divided into generalists, such as Calliphora vicina or Lucilia caesar, and specialists that occur in certain stages of decay, such as Hydrotaea aenescens or Hydrotaea meteorica (Matuszewski et al. 2010b). Both types of insects might identify core compounds of vertebrate decay, which occur at any time or in certain stages of decay. In general, insect antennae serve as information filters that help in identifying volatiles for different applications. For instance, a post-mortem interval-correlated qualitative and quantitative dynamic volatile pattern could give an estimate of the time since death (Statheropoulos et al. 2007; Vass et al. 2004) or selected volatiles may improve cadaver dog training (Hoffman et al. 2009). Dimethyl sulfide, dimethyl disulfide, and dimethyl trisulfide are already known as electrophysiologically active for the blowflies $C$. vicina and L. caesar (Stensmyr et al. 2002) and the burying beetles Nicrophorus vespillo and Nicrophorus vespilloides (Kalinova et al. 2009). Such volatiles should be reliable for routine application and could be detected by low-cost and compact trace analysis devices such as semi-conductor gas sensor systems (Kohl 2003; Paczkowski et al. 2011). The tracing of victims of natural disasters, such as those buried in earthquakes, as well as the tracking of victims of homicides whose bodies have been buried (Killam 2004; Statheropoulos et al. 2007; Vass et al. 2008) may be improved by such methods (Statheropoulos et al. 2006). The industrial application of a detector for early vertebrate post-mortem VOCs could help in determining the degree of spoilage or estimation of the storage time of fish and meat (Lindinger et al. 1998; Nicolay 2006; Winquist et al. 1993). 
Table 4 Contains important experimental parameters used in the studies listed in Table 3

\begin{tabular}{|c|c|c|c|c|}
\hline Number & Publication & Tissue & Location & Sampling technique \\
\hline 1 & Dekeirsschieter et al. 2009 & Domestic pig & Belgium, forest & Radiello, passive, 1 week, GC/MS \\
\hline 2 & Dekeirsschieter et al. 2009 & Domestic pig & Belgium, rural & Radiello, passive, 1 week, GC/MS \\
\hline 3 & Dekeirsschieter et al. 2009 & Domestic pig & Belgium, urban & Radiello, passive, 1 week, GC/MS \\
\hline 4 & Vass et al. 2004 & Human & $\begin{array}{l}\text { USA, Tennessee, } \\
\text { open woodland }\end{array}$ & $\begin{array}{l}\text { Triple sorbent traps (Carbotrap, } \\
\text { Carbotrap C, Carbosieve S-III), GC/MS }\end{array}$ \\
\hline 5 & Vass et al. 2008 & $\begin{array}{l}\text { Human, bones } \\
\text { of human, deer, dog }\end{array}$ & $\begin{array}{l}\text { USA, Tennessee, } \\
\text { open woodland }\end{array}$ & $\begin{array}{l}\text { Triple sorbent traps (Carbotrap, } \\
\text { Carbotrap C, Carbosieve S-III), GC/MS }\end{array}$ \\
\hline 6 & Statheropoulos et al. 2005 & Human & Greece, island of Samos & $\begin{array}{l}\text { Three-layer sampling sorbent glass tube } \\
\text { (Carbograph 1, Carbograph 2, } \\
\text { Carbosieve S-III), 51, GC/MS }\end{array}$ \\
\hline 7 & Statheropoulos et al. 2007 & Human & Greece, eastern Attica & $\begin{array}{l}\text { Three-layer sampling sorbend glass tube } \\
\text { (Carbopack B, Carbopack C, } \\
\text { Carbosieve S-III), 5l, GC/MS }\end{array}$ \\
\hline 8 & Hoffman et al. 2009 & Different human tissues & - & $\begin{array}{l}\text { SPME (PDMS/DVB), for } 20 \text { and } \\
40 \text { min in glas vial, GC/MS }\end{array}$ \\
\hline 9 & Haze et al. 2001 & Living human skin & Japan & $\begin{array}{l}\text { TENAX-TA column } \\
\text { (GL Science), 101, GC/MS }\end{array}$ \\
\hline 10 & Yan et al. 2001 & Adipocere & - & - \\
\hline 11 & Forbes et al. 2005a & Domestic pig adipocere & Australia, laboratory & $\begin{array}{l}1 \mu 1 \text { of chloroform extract, } \\
\text { HMDS esterification, GC/MS }\end{array}$ \\
\hline 12 & Forbes et al. $2005 b$ & Domestic pig adipocere & Australia, laboratory & $\begin{array}{l}1 \mu \mathrm{l} \text { of chloroform extract, } \\
\text { HMDS esterification, GC/MS }\end{array}$ \\
\hline 13 & Forbes et al. $2005 \mathrm{c}$ & Domestic pig adipocere & Australia, laboratory & $\begin{array}{l}1 \mu \mathrm{l} \text { of chloroform extract, } \\
\text { HMDS esterification, GC/MS }\end{array}$ \\
\hline 14 & Forbes et al. 2002 & Soil sample beneath coffin & Australia & $\begin{array}{l}1 \mu \mathrm{l} \text { of chloroform extract, } \\
\text { HMDS esterification, GC/MS }\end{array}$ \\
\hline 15 & Notter et al. 2009 & Domestic pig adipocere & Australia, laboratory & $1 \mu \mathrm{l}$ of derivatised sample, GC/MS \\
\hline 16 & Nushida et al. 2008 & Human adipocere & Japan, laboratory & Processed and derivatised sample, GC/MS \\
\hline 17 & Stutz et al. 1991 & Ground beef & Laboratory & Porapak trap, GC/MS \\
\hline 18 & Ercolini et al. 2009 & Beef muscle, latissimus dorsi & Laboratory & $\begin{array}{l}5 \mathrm{~g} \text { homogenized in } 100 \mathrm{ml} \text { water and } \\
30 \mathrm{~g} \mathrm{NaCl} \text {, SPME (CAR/DVB/PDMS) } \\
\text { for } 1 \mathrm{~h}, \mathrm{GC} / \mathrm{MS}\end{array}$ \\
\hline 19 & Zhang et al. 1992 & Ayu skin, arachidonic acid & Japan & $\begin{array}{l}30 \mathrm{ml}(1 \mathrm{mg} \text { protein } / \mathrm{ml})+50 \mu \mathrm{M} \\
\text { arachidonic acid; Tenax TA, } 70 \mathrm{ml} / \mathrm{min} \text {, } \\
90 \mathrm{~min}, \mathrm{GC} / \mathrm{MS}\end{array}$ \\
\hline 20 & Kalinova et al. 2009 & Mouse (Mus musculus) & Laboratory & $\begin{array}{l}\text { SPME }(\mathrm{CAR} / \mathrm{PDMS}) \text { for } 15 \mathrm{~min} \text { in } \\
\text { glass container, } \mathrm{GC} \times \mathrm{GC}-\mathrm{TOFMS}\end{array}$ \\
\hline
\end{tabular}

Although the formation of biogenic amines is known to be correlated with microbial growth on vertebrate tissue, most of these compounds have a low volatility and, therefore, are not useful for a volatile-based quality estimation. Histamine, putrescine, cadaverine, tyramine, tryptamine, $\beta$-phenylethylamine, spermine, and spermidine are reported to be the most important biogenic amines (Onal 2007). Especially putrescine and cadaverine were regarded as important volatile products of vertebrate decay; however, all of the listed amines have a low volatility and, therefore, are not frequently identified in analytical literature on cadaveric volatiles (Table 3). Together with lipid acid formation during the formation of adipocere, biogenic amines could be important in estimating the pmi by means of liquid chromatography or SPME liquid extraction (Onal 2007). However, gas sensor systems would most likely not be able to detect them. On the other hand, ammonia might play an important role in aging vertebrate tissue. Olafsdottir and Kristbersson (2006) discuss the performance of electronic noses for the quality estimation of fresh fish. As gas sensors are restricted in selecting single compounds from an odor, the authors refer to compound groups such as sulfur compounds, volatile amines like ammonia, esters, aldehydes, or alcohols. The quantitative emission of compounds from these groups did correlate with the signals of three gas sensors. Hence, fish storage time was 
detectable for the sensor array in this experiment. Winquist et al. used an array of 15 gas sensors detecting hydrogen, hydrogen sulfide, amines, alcohols, saturated hydrocarbons, and humidity in order to estimate the spoilage of ground beef (Winquist et al. 1993). In this study, a three-layerpattern recognition routine was used in order to train the electronic nose on the recognition of the type of meat and the storage time. It was possible to determine the type of meat and the storage time of ground pork and beef; however, the authors state that in this experiment variation was minimal in comparison to the potential variation in practice. The quantitative emissions of the compound classes may vary to a high extent and important volatile cues might be under the detection threshold for gas sensors. For instance, Lindinger et al. detected methanethiol in parts-per-billion concentrations in the headspace of beef meat stored at $22{ }^{\circ} \mathrm{C}$ (Lindinger et al. 1998). As already described in this review, methanethiol is a good candidate for meat spoilage detection; however, it has to be evaluated if gas sensors are able to detect the compound at such a concentration level. Furthermore, meat stored at chill temperatures will develop a psychrotrophic micro-fauna, consisting of lactic acid bacteria and Pseudomonas spp. or Enterobacteriaceae. The volatile profile of these bacteria will differ from the volatile profile of micro-organisms growing at ambient temperatures. Ercolini et al. investigated the odor profile of psychrotrophic micro-organisms and did not find methanethiol (Ercolini et al. 2009), which indicates that this compound might form only at elevated temperatures. Rajamaki et al. found an increasing quantity of dimethyl disulfide in the headspace of broiler chicken at an elevated temperature, which might have formed from methanethiol (Rajamaki et al. 2006). Hence, methanethiol or the associated methylated sulfides could be markers for the inappropriate storage of meat at elevated temperatures. In the study of Rajamäki et al., a commercial electronic nose with a principal component analysis detected hydrogen sulfide concentrations, which allowed one to determine spoilage on meat packed under a modified atmosphere (Rajamaki et al. 2006). However, gas sensor technology has to be improved to enable the detection of low concentrations of single compounds for a reliable spoilage estimation. This improvement can be achieved by immobilizing proteins on sensor surfaces to utilize the strong odor detection abilities in nature (Hou et al. 2005; Khanna et al. 2006) or by varying the operating temperature for enhanced gas sensor selectivity (Sauerwald et al. 2007).

\section{Conclusion}

This review highlights the VOC emissions of dead vertebrates based on data from experimental trace analysis and their formation. There is a correlation between the information on biochemical processes and phenomenological results. For some volatiles, the pathways of their formation are entirely unknown or not known in detail. In particular, the detailed processes of fat degradation in prokaryotes are unknown. Based on existing information in the literature, it may be possible to hypothesize a core pattern of volatile emission during decay. After death, the volatiles produced by ROS might be the first to give rise to changes, and this event would be accompanied by the increasing growth of microbes that metabolize the fat on the skin and lead to decomposition by the tissues' own enzymes. Once the immune system fails, the microbes that are already present inside the respiratory tract or in the gastrointestinal tract would contribute to the volatile pattern of decay. The metabolic products of particular intestinal microbes cause bloating under anaerobic conditions. This influences the microbial species composition and therefore gives rise to other volatile metabolic products. Breakage of the skin leads to the entry of oxygen, which again favors different microbes, yielding different volatiles. In an oxidative environment, secondary volatile products might form. During advanced dry decay, most of the carbohydrates and proteins are degraded. The volatiles produced by ROS and the microbial decomposition of bone components might dominate at this stage. Throughout this process, microbes might interact and thereby influence the pattern of the volatiles released. At present, information on vertebrate decay is incomplete. Therefore, the idea of a core pattern of volatiles of vertebrate decay remains a hypothesis. Further research on the application of post-mortem volatiles should integrate the knowledge of microbiologists and forensic scientists in order to perform experiments on the biochemical origin of these volatiles. The evolutional preadaption of insect olfaction to the perception of decompositional volatiles may help in developing volatile-based methods for forensic science, such as postmortem time estimation or cadaver dog training.

Acknowledgement The Cusanuswerk, Bischöfliche Studienstiftung, kindly supports the work of Sebastian Paczkowski.

Open Access This article is distributed under the terms of the Creative Commons Attribution Noncommercial License which permits any noncommercial use, distribution, and reproduction in any medium, provided the original author(s) and source are credited.

\section{References}

Alexieva Z, Gerginova M, Manasiev J, Zlateva P, Shivarova N, Krastanov A (2008) Phenol and cresol mixture degradation by the yeast Trichosporon cutaneum. J Ind Microbio Biot 35:1297-1301

Amendt J, Krettek R, Zehner R (2004) Forensic entomology. Naturwissenschaften 91:51-65

Ansorena D, Montel MC, Rokka M, Talon R, Eerola S, Rizzo A, Raemaekers M, Demeyer D (2002) Analysis of biogenic amines 
in northern and southern European sausages and role of flora in amine production. Meat Sci 61:141-147

Arp DJ (1999) Butane metabolism by butane-grown Pseudomonas butanovora. Microbiol-UK 145:1173-1180

Bone E, Tamm A, Hill M (1976) Production of urinary phenols by gut bacteria and their possible role in causation of large bowel cancer. Am J Clin Nutr 29:1448-1454

Borjesson TS, Stollman UM, Schnurer JL (1993) Off-odorous compounds produced by molds on oatmeal agar-identification and relation to other growth-characteristics. J Agr Food Chem 41:2104-2111

Boschker HTS, Middelburg JJ (2002) Stable isotopes and biomarkers in microbial ecology. FEMS Microbiol Ecol 40:85-95

Boumba VA, Ziavrou KS, Vougiouklakis T (2008) Biochemical pathways generating post-mortem volatile compounds co-detected during forensic ethanol analyses. Forensic Sci Int 174:133-151

Branscheid W, Honikel KO, vLengerken G, Troeger K (2007) Qualität von Fleisch- und Fleischwaren. Deutscher Fachverlag, Frankfurt

Bryant MP, Wolin EA, Wolin MJ, Wolfe RS (1967) Methanobacillus omelianskii, a symbiotic association of 2 species of bacteria. Arch Mikrobiol 59:20-31

Cadenas E (1989) Biochemistry of oxygen-toxicity. Annu Rev Biochem 58:79-110

Carter DO, Yellowlees D, Tibbett M (2007) Cadaver decomposition in terrestrial ecosystems. Naturwissenschaften 94:12-24

Chang J, Kollman SE (1989) The effect of temperature on the formation of ethanol by Candida-albicans in blood. J Forensic Sci 34:105-109

Chen ECH (1978) Relative contribution of Ehrlich and biosynthetic pathways to the formation of fusel alcohols. J Am Soc Brew Chem 36:39-43

Choe E, Min DB (2005) Chemistry and reactions of reactive oxygen species in foods. J Food Sci 70:142-159

Combet E, Henderson J, Eastwood CD, Burton KS (2006) Eightcarbon volatiles in mushrooms and fungi: properties analysis and biosynthesis. Mycoscience 47:317-326

Corry JEL (1978) Possible sources of ethanol ante-mortem and postmortem - its relationship to biochemistry and microbiology of decomposition. J Appl Bacteriol 44:1-56

Cummings JH, Hill MJ, Bone ES, Branch WJ, Jenkins DJA (1979) Effect of meat protein and dietary fiber on colonic function and metabolism. 2. Bacterial metabolites in feces and urine. Am J Clin Nutr 32:2094-2101

Dainty RH, Edwards RA, Hibbard CM (1985) Time course of volatile compound formation during refrigerated storage of naturally contaminated beef in air. J Appl Bacteriol 59:303-309

Dainty RH, Edwards RA, Hibbard CM, Marnewick JJ (1989) Volatile compounds associated with microbial-growth on normal and high ph beef stored at chill temperatures. J Appl Bacteriol 66:281-289

Davis GL, Leffert RL, Rantanen NW (1972) Putrefactive ethanol sources in postmortem tissues of conventional and germ-free mice. Arch Pathol 94:71-74

Dekeirsschieter J, Verheggen FJ, Gohy M, Hubrecht F, Bourguignon L, Lognay G, Haubruge E (2009) Cadaveric volatile organic compounds released by decaying pig carcasses (Sus domesticus L.) in different biotopes. Forensic Sci Int 189:46-53

Demeyer D, Vannevel C (1979) Protein fermentation and growth by rumen microbes. Ann Rech Vet 10:277-279

Dent BB, Forbes SL, Stuart BH (2004) Review of human decomposition processes in soil. Environ Geol 45:576-585

Derrick S, Large PJ (1993) Activities of the enzymes of the Ehrlich pathway and formation of branched-chain alcohols in Saccharomyces-cerevisiae and Candida-utilis grown in continuous-culture on valine or ammonium as sole nitrogen-source. J Gen Microbiol 139:2783-2792

Dotterl S, Burkhardt D, Weissbecker B, Jurgens A, Schutz S, Mosandl A (2006) Linalool and lilac aldehyde/alcohol in flower scents- electrophysiological detection of lilac aldehyde stereoisomers by a moth. J Chromatogr A 1113:231-238

Dransfield E (1992) Modeling postmortem tenderization. 3. Role of calpain-I in conditioning. Meat Sci 31:85-94

Durlu-Özkaya F, Ayhan K, Vural N (2001) Biogenic amines produced by Enterobacteriaceae isolated from meat products. Meat Sci $58: 163-166$

Dutson TR (1983) Relationship of ph and temperature to disruption of specific muscle proteins and activity of lysosomal proteases. J Food Biochem 7:223-245

Ercolini D, Russo F, Nasi A, Ferranti P, Villani F (2009) Mesophilic and psychrotrophic bacteria from meat and their spoilage potential in vitro and in beef. Appl Environ Microb 75:1990-2001

Estevez M, Morcuende D, Ventanas S, Cava R (2003) Analysis of volatiles in meat from Iberian pigs and lean pigs after refrigeration and cooking by using SPME-GC-MS. J Agr Food Chem 51:3429-3435

Evans WC (1963) Microbiological degradation of aromatic compounds. J Gen Microbiol 32:177-184

Evershed RP, Turnerwalker G, Hedges REM, Tuross N, Leyden A (1995) Preliminary-results for the analysis of lipids in ancient bone. J Archeol Sci 22:277-290

Fernando WGD, Ramarathnam R, Krishnamoorthy AS, Savchuk SC (2005) Identification and use of potential bacterial organic antifungal volatiles in biocontrol. Soil Biol Biochem 37:955-964

Feussner I, Kühn H, Wasternack C (1997) Do specific linoleate 13lipoxygenases initiate B-oxidation? FEBS Lett 406:1-5

Forbes SL, Stuart BH, Dent BB (2002) The identification of adipocere in grave soils. Forensic Sci Int 127:225-230

Forbes SL, Dent BB, Stuart BH (2005a) The effect of soil type on adipocere formation. Forensic Sci Int 154:35-43

Forbes SL, Stuart BH, Dent BB (2005b) The effect of the burial environment on adipocere formation. Forensic Sci Int 154:24-34

Forbes SL, Stuart BH, Dent BB (2005c) The effect of the method of burial on adipocere formation. Forensic Sci Int 154:44-52

Frankel EN (1983) Volatile lipid oxidation-products. Prog Lipid Res 22:1-33

Frederick LR, Starkey RL, Segal W (1957) Decomposability of some organic sulphur compounds in soil. P Soil Sci Soc Am 21:287-292

Freney JR (1967) Sulphur-containing organics. In: Mac Laren AD, Peterson GH (eds) Soil biochemistry. Marcel Dekker, New York, pp 229-259

Gandemer G (2002) Lipids in muscles and adipose tissues changes during processing and sensory properties of meat products. Meat Sci 62:309-321

Geypens B, Claus D, Evenepoel P, Hiele M, Maes B, Peeters M, Rutgeerts P, Ghoos Y (1997) Influence of dietary protein supplements on the formation of bacterial metabolites in the colon. Gut 41:70-76

Gilbert RG, Menzies JD, Griebel GE (1969) Influence of volatiles from alfalfa upon growth and survival of soil microorganisms. Phytopathology 59:992-995

Gill CO, Penney N, Nottingham PM (1976) Effect of delayed evisceration on microbial quality of meat. Appl Environ Microbiol 31:465-468

Gill-King H (1999) Chemical and ultrastructural aspects of decomposition. In: Haglund WD, Sorg MH (eds) Forensic taphonomy. The postmortem fate of human remains. CRC, Boca Raton, pp 93-108

Goll DE, Otsuka Y, Nagainis PA, Shannon JD, Sathe SK, Muguruma M (1983) Role of muscle proteinases in maintenance of muscle integrity and mass. J Food Biochem 7:137-177

Gottschalk G (1986) Bacterial metabolism. Springer, New York

Gray TRG, Williams ST (1971) Soil microorganisms. Oliver and Boyd, Edinburgh

Gu YQ, Mo MH, Zhou JP, Zou CS, Zhang KQ (2007) Evaluation and identification of potential organic nematicidal volatiles from soil bacteria. Soil Biol Biochem 39:2567-2575 
Gulzow HJ (1982) On the anaerobic transformation of palatinit by microorganisms of the human oral cavity. Deut Zahnarztl Z 37:669-672

Gurr MI (1988) Lipid-metabolism in man. P Nutr Soc 47:277-285

Haagsma J (1991) Pathogenic anaerobic bacteria and the environment. Rev Sci Tech 10:749-764

Haglund WD (1997) Dogs and coyotes: postmortem involvement with human remains. In: Haglund WD, Sorg MH (eds) Forensic taphonomy: the postmortem fate of human remains. CRC, Boca Raton, pp 367-382

Haze S, Gozu Y, Nakamura S, Kohno Y, Sawano K, Ohta H, Yamazaki K (2001) 2-Nonenal newly found in human body odor tends to increase with aging. J Invest Dermatol 116:520-524

Heath RJ, Jackowski S, Rock CO (2002) Fatty acid and phospholipid metabolism in prokaryotes. In: Vance DE, Vance JE (eds) Biochemistry of lipids, lipoproteins and membranes. Elsevier, Amsterdam, pp 55-92

Herrera MC, Duque E, Rodriguez-Herva JJ, Fernandez-Escamilla AM, Ramos JL (2010) Identification and characterization of the PhhR regulon in Pseudomonas putida. Environ Microbiol 12:1427-1438

Higgins IJ, Burns RG (1975) The chemistry and microbiology of pollution. Academic, London

Hoffman EM, Curran AM, Dulgerian N, Stockham RA, Eckenrode BA (2009) Characterization of the volatile organic compounds present in the headspace of decomposing human remains. Forensic Sci Int 186:6-13

Hopkins DW, Wiltshire PEJ, Turner BD (2000) Microbial characteristics of soils from graves: arm investigation at the interface of soil microbiology and forensic science. Appl Soil Ecol 14:283-288

Hou YX, Jaffrezic-Renault N, Martelet C, Tlili C, Zhang A, Pernollet JC, Briand L, Gomila G, Errachid A, Samitier J, Salvagnac L, Torbiero B, Temple-Boyer P (2005) Study of Langmuir and Langmuir-Blodgett films of odorant-binding protein/amphiphile for odorant biosensors. Langmuir 21:4058-4065

Hübschmann H-J (2009) Handbook of GC/MS. Wiley-VCH, Weinheim

Huotari M, Mela M (1996) Blowfly olfactory biosensor's sensitivity and specificity. Sensors Actuat B-Chem 34:240-244

Iannotti EL, Kafkewit D, Wolin MJ, Bryant MP (1973) Glucose fermentation products of Ruminococcus-albus grown in continuous culture with Vibrio succinogenes - changes caused by interspecies transfer of $\mathrm{H}_{2}$. J Bacteriol 114:1231-1240

Insausti K, Goni V, Petri E, Gorraiz C, Beriain MJ (2005) Effect of weight at slaughter on the volatile compounds of cooked beef from Spanish cattle breeds. Meat Sci 70:83-90

Ito T, Ando T, Mayahara H, Miyajima H, Ogawa K (1991a) Postmortem changes in the rat-kidney. 1. Histopathological electron-microscopic and enzyme histochemical-studies of postmortem changes at roomtemperature. Acta Histochem Cytoc 24:135-151

Ito T, Ando T, Mayahara H, Miyajima H, Ogawa K (1991b) Postmortem changes in the rat-kidney. 2. Histopathological electron-microscopic and enzyme histochemical-studies of postmortem changes at 0-degrees-C. Acta Histochem Cytoc 24:153166

Jay JM (2000) Food preservation with chemicals. Aspen, Gaithersburg

Jenny J (1983) Bacteriology, mycology and parasitology of the vaginafrequency and clinical-significance of the different microorganisms. Schweiz Rundsch Med 72:1515-1520

Kadota HG, Ishida Y (1972) Production of volatile sulphur compounds by micro-organisms. Ann Revf Microbiol 26:127-138

Kai M, Haustein M, Molina F, Petri A, Scholz B, Piechulla B (2009) Bacterial volatiles and their action potential. Appl Microbiol Biotechnol 81:1001-1012

Kaib M (1974) Receptors for meat-odor and flower-odor on antenna of blowfly Calliphora vicina. J Comp Phys 95:105-121

Kalinova B, Podskalska H, Ruzicka J, Hoskovec M (2009) Irresistible bouquet of death - how are burying beetles (Coleoptera: Silphi- dae: Nicrophorus) attracted by carcasses. Naturwissenschaften 96:889-899

Khanna VK, Kumar A, Jain YK, Ahmad S (2006) Design and development of a novel high-transconductance pH-ISFET (ionsensitive field-effect transistor)-based glucose biosensor. Int $\mathrm{J}$ Electron 93:81-96

Killam EW (2004) The detection of human remains. Thomas, Springfield

King JE, Coley-Smith JR (1969) Production of volatile alkyl sulphides by microbial degradation of synthetic alliin and alliin-like compounds in relation to germination of sclerotia of Sclerotium cepivorum Berk. Ann Appl Biol 64:303-314

Kionka C, Kunau WH (1985) Inducible beta-oxidation pathway in Neurospora-crassa. J Bacteriol 161:153-157

Kohl C-D (2003) Electronic noses. In: Waser R (ed) Nanoelectronics and information technology-advanced electronic materials and novel devices. Wiley-VCH, Berlin

Koohmaraie M (1992) The role of $\mathrm{Ca}(2+)$-dependent proteases (calpains) in postmortem proteolysis and meat tenderness. Biochimie 74:239 245

Koohmaraie M (1994) Muscle proteinases and meat aging. Meat Sci 36:93-104

Korpi A, Pasanen AL, Pasanen P (1998) Vblatile compounds originating from mixed microbial cultures on building materials under various humidity conditions. Appl Environ Microbiol 64:2914-2919

Labows JL (1982) Skin microorganisms and body odors. J Soc Cosmet Chem 33:369

Larsen TO, Frisvad JC (1995) Comparison of different methods for collection of volatile chemical markers from fungi. J Microbiol Meth 24:135-144

LeBlanc HN, Logan JG (2010) Exploiting insect olfaction in forensic entomology. In: Amendt J, Campobasso CP, Goff ML, Grassberger $M$ (eds) Current concepts in forensic entomology. Springer, New York, pp 205-222

Lee JH, Waller JC, Melton SL, Saxton AM, Pordesimo LO (2004) Feeding encapsulated ground full-fat soybeans to increase polyunsaturated fat concentrations and effects on flavor volatiles in fresh lamb. J Anim Sci 82:2734-2741

Lestremau F, Andersson FAT, Desauziers V (2004) Investigation of artefact formation during analysis of volatile sulphur compounds using solid phase microextraction (SPME). Chromatographia 59:607-613

Lewis JA, Papavizas GC (1972) Evolution of volatile sulphurcontaining compounds from decomposition of crucifers in soil. Soil Biol Biochem 2:239-246

Lindinger W, Hansel A, Jordan A (1998) Proton-transfer-reaction mass spectrometry (PTR-MS): on-line monitoring of volatile organic compounds at pptv levels. Chem Soc Rev 27:347-354

Lopez-Caballero ME, Sanchez-Fernandez JA, Moral A (2001) Growth and metabolic activity of Shewanella putrefaciens maintained under different $\mathrm{CO}_{2}$ and $\mathrm{O}_{2}$ concentrations. Int J Food Microbiol 64:277-287

Lorenzo N, Wan TL, Harper RJ, Hsu YL, Chow M, Rose S, Furton KG (2003) Laboratory and field experiments used to identify canis lupus var. familiaris active odor signature chemicals from drugs, explosives, and humans. Anal Bioanal Chem 376:12121224

Luengo JM, Garcia JL, Olivera ER (2001) The phenylacetyl-CoA catabolon: a complex catabolic unit with broad biotechnological applications. Mol Microbiol 39:1434-1442

Macfarlane GT, Allison C (1986) Utilisation of protein by human gut bacteria. FEMS Microbiol Ecol 38:19-24

Marinho AND, Miranda NC, Braz V, Ribeiro-dos-Santos AK, deSouza SMFM (2006) Paleogenetic and taphonomic analysis of human bones from Moa Beirada and Ze Espinho Sambaquis Rio de Janeiro Brazil. Mem do I Oswaldo Cruz 101:15-23

Matuszewski S, Bajerlein D, Konwerski S, Szpila K (2010a) Insect succession and carrion decomposition in selected forests of 
Central Europe. Part 1: pattern and rate of decomposition. Forensic Sci Int 194:85-93

Matuszewski S, Bajerlein D, Konwerski S, Szpila K (2010b) Insect succession and carrion decomposition in selected forests of Central Europe. Part 2: composition and residency patterns of carrion fauna. Forensic Sci Int 195:42-51

Meier-Augenstein W (2002) Stable isotope analysis of fatty acids by gas chromatography-isotope ratio mass spectrometry. Anal Chim Acta 465:63-79

Michaud JP, Moreau G (2011) A statistical approach based on accumulated degree-days to predict decomposition-related processes in forensic studies. J Forensic Sci 56:229-232

Miller WC, Palmer WK, Arnall DA, Oscai LB (1987) Characterization of the triacylglycerol lipase activity in 3 types of rat skeletalmuscle. Can J Physiol Pharm 65:317-322

Molin G, Ternstrom A (1986) Phenotypically based taxonomy of psychrotrophic Pseudomonas isolated from spoiled meat water and soil. Int J Syst Bacteriol 36:257-274

Moller JKS, Hinrichsen LL, Andersen HJ (1998) Formation of amino acid (L-leucine L-phenylalanine) derived volatile flavour compounds by Moraxella phenylpyruvica and Staphylococcus xylosus in cured meat model systems. Int J Food Microbiol 42:101-117

Moore WEC, Holdeman LV (1974) Human fecal flora-normal flora of 20 Japanese-Hawaiians. Appl Microbiol 27:961-979

Moreno-Opo R, Margalida A, Arredondo A, Guil F, Martin M, Higuero R, Soria C, Guzman J (2010) Factors influencing the presence of the cinereous vulture Aegypius monachus at carcasses: food preferences and implications for the management of supplementary feeding sites. Wildlife Biol 16:25-34

Motilva MJ, Toldra F, Nieto P, Flores J (1993) Muscle lipolysis phenomena in the processing of dry-cured ham. Food Chem 48:121-125

Negre AE, Salvayre RS, Dagan A, Gatt S (1985) New fluorometric assay of lysosomal acid lipase and its application to the diagnosis of Wolman and cholesteryl ester storage diseases. Clin Chim Acta 149:81-88

Nicolay X (2006) Odors in the food industry. Springer, New York

Notter SJ, Stuart BH, Rowe R, Langlois N (2009) The initial changes of fat deposits during the decomposition of human and pig remains. J Forensic Sci 54:195-201

Nushida H, Adachi J, Takeuchi A, Asano M, Ueno Y (2008) Adipocere formation via hydrogenation of linoleic acid in a victim kept under dry concealment. Forensic Sci Int 175:160-165

Okuda H (1991) Lipases. In: Kuby SA (ed) A study of enzyme action. CRC, Boca Raton, pp 579-593

Olafsdottir G, Kristbergsson K (2006) Electronic-nose technology: application for quality evaluation in the fish industry. In: Nicolay $\mathrm{X}$ (ed) Odors in the food industry. Springer, New York, pp 57-74

Onal A (2007) A review: current analytical methods for the determination of biogenic amines in foods. Food Chem 103:1475-1486

O`Neal CL, Poklis A (1996) Postmortem production of ethanol and factors that influence interpretation - a critical review. Am J Foren Med Path 17:8-20

Paczkowski S, Weißbecker B, Schöning MJ, Schütz S (2011) Biosensors on the basis of insect olfaction. In: Vilcinskas A (ed) Insect biotechnology. Springer, Dordrecht, pp 225-240

Pasanen AL, Lappalainen S, Pasanen P (1996) Volatile organic metabolites associated with some toxic fungi and their mycotoxins. Analyst 121:1949-1953

Patel RN, Hou CT, Laskin AI, Derelanko P, Felix A (1979) Microbialproduction of methyl ketones - purification and properties of a secondary alcohol-dehydrogenase from yeast. Eur J Biochem 101:401-406

Patel RN, Hou CT, Laskin AI, Felix A, Derelanko P (1980) Microbial oxidation of gaseous hydrocarbons - production of secondary alcohols from corresponding normal-alkanes by methaneutilizing bacteria. Appl Environ Microbiol 39:720-726

Polson CJ, Gee DJ, Knight B (1985) The essentials of forensic medicine. Pergamon, Oxford

Rabus R, Heider J (1998) Initial reactions of anaerobic metabolism of alkylbenzenes in denitrifying and sulphate reducing bacteria. Arch Microbiol 170:377-384

Rajamaki T, Alakomi HL, Ritvanen T, Skytta ES, Smolander M, Ahvenainen R (2006) Application of an electronic nose for quality assessment of modified atmosphere packaged poultry meat. Food Control 17:5-13

Ravaglioli A, Krajewski A, Celotti GC, Piancastelli A, Bacchini B, Montanari L, Zama G, Piombi L (1996) Mineral evolution of bone. Biomaterials 17:617-622

Sando GN, Rosenbaum LM (1985) Human lysosomal acid lipase cholesteryl ester hydrolase-purification and properties of the form secreted by fibroblasts in microcarrier culture. J Biol Chem 260:5186-5193

Sauerwald T, Skiera D, Kohl CD (2007) Selectivity enhancement of gas sensors using non-equilibrium polarisation effects in metal oxide films. Appl Phys A-Mater 87:525-529

Schultz M (2006) Microscopic structure of bone. In: Haglund WD, Sorg MH (eds) Forensic taphonomy. The postmortem fate of human remains. CRC, Boca Raton, pp 187-200

Schütz S, Weissbecker B, Hummel HE, Apel KH, Schmitz H, Bleckmann H (1999) Insect antenna as a smoke detector. Nature 398:298-299

Sink JD (1979a) Factors influencing the flavour of muscle foods. J Food Sci 44:1-5

Sink JD (1979b) Symposium on meat flavor factors influencing flavor of muscle foods. J Food Sci 44:1-5

Smith KGV (1986) A manual of forensic entomology. Department of Entomology, British Museum (Natural History), London

Smith EA, Macfarlane GT (1996) Enumeration of human colonic bacteria producing phenolic and indolic compounds: effects of $\mathrm{pH}$ carbohydrate availability and retention time on dissimilatory aromatic amino acid metabolism. J Appl Bacteriol 81:288-302

Sos J, Kemeny T, Rigo J, Budavari I (1961) Influence of amino acid deficiency on chemical constitution and solidity of bones. Acta Physiol Hung 19:267

Stahnke LH (1999) Volatiles produced by Staphylococcus xylosus and Staphylococcus carnosus during growth in sausage minces-part II. The influence of growth parameters. Food Sci Technol-LEB 32:365-371

Stams AJM (1994) Metabolic interactions between anaerobic-bacteria in methanogenic environments. Antonie Leeuw Int J G 66:271-294

Statheropoulos M, Spiliopouiou C, Agapiou A (2005) A study of volatile organic compounds evolved from the decaying human body. Forensic Sci Int 153:147-155

Statheropoulos M, Mikedi K, Agapiou A, Georgiadou A, Karma S (2006) Discriminant analysis of volatile organic compounds data related to a new location method of entrapped people in collapsed buildings of an earthquake. Anal Chim Acta 566:207-216

Statheropoulos M, Agapiou A, Spiliopouiou C, Pallis GC, Sianos E (2007) Environmental aspects of VOCs evolved in the early stages of human decomposition. Sci Total Environ 385:221-227

Stensmyr MC, Urru I, Collu I, Celander M, Hansson BS, Angioy AM (2002) Rotting smell of dead-horse arum florets - these blooms chemically fool flies into pollinating them. Nature 420:625-626

Straehlerpohl HJ, Exner M (1985) A comparative-study of the aerobic microorganisms of the oral cavity and nasopharynx cholesteatomas. Arch Oto-Rhino-Laryn 241:120

Stutz HK, Silverman GJ, Angelini P, Levin RE (1991) Bacteria and volatile compounds associated with ground-beef spoilage. J Food Sci 56:1147-1153 
Sunesson AL, Nilsson CA, Andersson B, Blomquist G (1996) Volatile metabolites produced by two fungal species cultivated on building materials. Ann Occup Hyg 40:397-410

Thakeow P, Angeli S, Weissbecker B, Schutz S (2008) Antennal and behavioral responses of Cis boleti to fungal odor of Trametes gibbosa. Chem Senses 33:379-387

Tholl D, Boland W, Hansel A, Loreto F, Rose USR, Schnitzler JP (2006) Practical approaches to plant volatile analysis. Plant J 45:540-560

Toldra F (1998) Proteolysis and lipolysis in flavour development of dry-cured meat products. Meat Sci 49:S101-S110

Vanderzant C, Savell JW, Hanna MO, Potluri V (1986) A comparison of growth of individual meat bacteria on the lean and fatty tissue of beef pork and lamb. J Food Sci 51:5-8

Vanklinken GJ, Hedges REM (1992) Experiments on C-14 dating of contaminated bone using peptides resulting from enzymatic cleavage of collagen. Radiocarbon 34:292-295

Vanklinken GJ, Bowles AD, Hedges REM (1994) Radiocarbon dating of peptides isolated from contaminated fossil bone-collagen by collagenase digestion and reversed-phase chromatography. Geochim Cosmochim Ac 58:2543-2551

Vass AA, Barshick SA, Sega G, Caton J, Skeen JT, Love JC, Synstelien JA (2002) Decomposition chemistry of human remains: a new methodology for determining the postmortem interval. J Forensic Sci 47:542-553

Vass AA, Smith RR, Thompson CV, Burnett MN, Wolf DA, Synstelien JA, Dulgerian N, Eckenrode BA (2004) Decompositional odor analysis database. J Forensic Sci 49:760-769

Vass AA, Smith RR, Thompson CV, Burnett MN, Dulgerian N, Eckenrode BA (2008) Odor analysis of decomposing buried human remains. J Forensic Sci 53:384-391

Vogt RG, Riddiford LM (1981) Pheromone binding and inactivation by moth antennae. Nature 293:161-163
Waksman SA, Starkey RL (1931) The soil and the microbe. Wiley, New York

Weissbecker B, Holighaus G, Schütz S (2004) Gas chromatography with mass spectrometric and electroantennographic detection: analysis of wood odorants by direct coupling of insect olfaction and mass spectrometry. J Chromatogr A 1056:209-216

Wells CL, Maddaus MA, Simmons RL (1988) Proposed mechanisms for the translocation of intestinal bacteria. Rev Infect Dis 10:958979

Wicher D, Schafer R, Bauernfeind R, Stensmyr MC, Heller R, Heinemann SH, Hansson BS (2008) Drosophila odorant receptors are both ligand-gated and cyclic-nucleotide-activated cation channels. Nature 452:1007-1010

Winquist F, Hornsten EG, Sundgren H, Lundstrom I (1993) Performance of an electronic nose for quality estimation of ground meat. Meas Sci Technol 4:1493-1500

Wolle DD, Banavara DS, Rankin SA (2006) Short communication: empirical and mechanistic evidence for the role of pyridoxal-5'phosphate in the generation of methanethiol from methionine. $J$ Dairy Sci 89:4545-4550

Yan F, Mcnally R, Kontanis EJ, Sadik OA (2001) Preliminary quantitative investigation of postmortem adipocere formation. $\mathrm{J}$ Forensic Sci 46:609-614

Yeung DKW, Lam SL, Griffith JF, Chan ABW, Chen ZY, Tsang PH, Leung PC (2008) Analysis of bone marrow fatty acid composition using high-resolution proton NMR spectroscopy. Chem Phys Lipids 151:103-109

Young LY, Rivera MD (1985) Methanogenic degradation of 4 phenolic-compounds. Water Res 19:1325-1332

Zhang CH, Hirano T, Suzuki T, Shirai T (1992) Studies on the odor of fishes. 2. Enzymatically generated specific volatile compounds in ayu tissues. Nippon Suisan Gakk 58:559-565 


\author{
Sebastian Paczkowski, ${ }^{1}$ M.Sc.; Friederike Maibaum, ${ }^{1}$ M.Sc.; Marta Paczkowska, ${ }^{1}$ M.Sc.; \\ and Stefan Schütz, ${ }^{1}$ Ph.D.
}

\title{
Decaying Mouse Volatiles Perceived by Calliphora vicina Rob.-Desv.*,†
}

\begin{abstract}
Volatiles emitted by decaying human remains are in the focus of recent research. The identification of core volatiles in this field is of high importance, because cadaveric volatiles generally show high variation. In this study, the volatile profiles of five mice (Myodes glareolus) were sampled with charcoal filter tubes from their time of death until advanced decay. Eleven compounds were quantitated by means of gas chromatography-mass spectrometry. Electroantennographic experiments with female Calliphora vicina antennae led to the identification of dimethyl trisulfide, dimethyl disulfide, nonanal, hexan-1-ol, 1-octen-3-ol, 3-methylbutan-1-ol, and heptanal as electrophysiologically active compounds. When these were compared, dimethyl trisulfide $(17 \mathrm{ng} / \mu \mathrm{L})$ and dimethyl disulfide $(11 \mathrm{ng} / \mu \mathrm{L})$ were found to be emitted in higher concentrations. The roles of these compounds and nonanal as core volatiles for cadaver detection or postmortem time determination and their correlation to the stages of decay and the accumulated degree days are discussed.
\end{abstract}

KEYWORDS: forensic science, forensic chemoecology, forensic entomology, Calliphora vicina, electroantennogram, decomposition process, volatile emission pattern, stages of decay, odor analysis, postmortem time, core compounds, accumulated degree days

Vertebrates are degraded by autolytic and microbial processes after their death (1-11). The chemical reactions catalyzed by the tissue's own enzymes, as well as microbial enzymes, yield volatile organic compounds as byproducts or end products $(9,12-20)$. These volatile compounds are currently being researched and considered as possible applications in forensic investigations. In 2004, Dent et al. (19) published a review of human decomposition processes in soil. In the same year, Vass et al. (14) introduced a postmortem volatile database for the purpose of developing a portable sensor capable of detecting buried remains. Dent et al. (19) discuss the likely pathways of protein, fat, carbohydrate, and bone decomposition, while Vass et al. (14) provide a first analysis on the volatile profile of human decomposition in graves. Both groups showed the existence of a dynamic in the volatile pattern.

Statheropoulos et al. (16) were the first to present experimental data on the volatiles emitted by human remains on the soil surface at a specific time point in the decaying process. In 2007, Statheropoulos et al. (15) published the quantitative dynamic of the volatile pattern in the early stages of human decomposition. This discusses the practical use of the volatile pattern of human decay for canine training or the detection of human remains. In 2009, Dekeirsschieter et al. (12) sampled the volatile profile of pigs in three separate habitats. They discuss the influence of factors affecting the decompositional process and proposed a common

\footnotetext{
${ }^{1}$ Department of Forest Zoology and Forest Conservation, Büsgeninstitut, Georg-August-University, Göttingen, Germany.

* Presented in part at the 7th Annual Meeting of the European Association of Forensic Entomology, June 9-12, 2009, in Uppsala, Sweden.

Support provided by the Cusanuswerk, Bischöfliche Studienstiftung, Germany.

Received 30 Nov. 2010; and in revised form 15 July 2011; accepted 7 Aug. 2011.
}

core of volatiles, which were identified in all habitats. A study by Hoffman et al. (17) shows similarities across various regions and different tissues of decaying remains. All of these studies found a high variability of the volatile pattern of decaying vertebrates and discuss constant dynamics in the volatile emissions.

The general problem seems to be the identification of core volatiles by means of common trace analytical methods. They should have three characteristics: First, they should be removed from as much variation as possible, enabling cadaver dogs to find human remains despite disparities in climate, habitat, or condition of the cadaver. A portable sensor system for tracing cadavers or postmortem interval (PMI) estimation would also rely on these characteristics. Second, the volatiles should be emitted in high quantities, as cadaver dogs are then able to trace them over larger distances, and gas sensor applications have a relatively high detection threshold. Third, the emitted quantities of the core volatiles should show a constant dynamic to correlate them to the process of decay. Such a correlation could include several volatiles, some typical for the early stages of decay, and some typical for the later stages.

To identify the core volatiles in question, it was decided that mice (Myodes glareolus) would be used in the experiments. Core volatiles should form from basic chemical compounds such as those found in muscle tissue or neurons; therefore, a constant dynamic should be detectable in mice as well as in larger vertebrates. A small cadaver also emits less volatiles; hence, the selection of volatiles with the listed characteristics should be easier. However, any realistic experimental design would most probably not allow validating core volatiles. Therefore, it was decided that the female blowfly Calliphora vicina would be used as an information filter for the scent of decay. The fly feeds and oviposits on deceased vertebrates (21-23). Ovipositing begins 
shortly after death and continues until advanced decay $(22,23)$; therefore, this fly is a generalist among carrion visitors. Its olfaction might be adapted to perceive core volatiles, which would allow tracking of dead vertebrates for ovipositing.

The biochemistry of insect olfaction is different from the olfaction in mammals and not yet completely understood. The most accepted hypothesis is that the odor molecules enter specialized olfactory sensillae on the surface of an insect antenna. Here, they are bound to odorant binding proteins. This binding complex is able to activate olfactory receptors on the surface of axons inside the sensillae, which can lead to an electrophysiological reaction (24). In two separate studies, Kaib (25) and Huotari and Mela (26) identified several compounds, which were electrophysiologically active on the antenna of $C$. vicina. In both studies, the compounds were not identified as products of vertebrate decay, but chosen randomly. Compounds such as dimethyl disulfide or dimethyl trisulfide, which occur frequently in recent literature as byproducts of vertebrate decomposition, were not tested during these earlier studies. The first study, combining trace analysis of dead vertebrates with electrophysiology on C. vicina, was performed by Stensmyr et al. (20). They identified dimethyl sulfide, dimethyl disulfide, and dimethyl trisulfide as active on the antenna of the blowflies C. vicina and Lucilia caesar. However, they sampled the decaying vertebrate only once and did not provide information on its stage of decay. To fill this gap, the volatile patterns of five decaying mice were analyzed. Thereby, the antenna of $C$. vicina was used as a phylogenetically adapted information filter to select core volatiles of vertebrate decay.

\section{Materials and Methods}

\section{Collection of Volatiles}

During the late summer of 2008, five mice (M. glareolus) were placed in a deciduous forest near Göttingen, Lower Saxony, Germany. The mice were caught in the forest in live traps to avoid the killing of preserved mouse species. The traps were controlled twice a day, and mice of the species M. glareolus were captured and killed using $\mathrm{CO}_{2}$. Approval from the university's Animal Ethics Committee was obtained prior to trapping the mice. Wild animals were used so as to allow a natural variation of the chemical composition of the mouse tissues. The mouse cadavers were placed inside casings made from wire and fixed to the ground with tent pegs to protect them against scavengers. To collect the volatiles, the dead mice were placed in 1.5-L polyethylene bags (Confresco Frischhalteprodukte, Minden, Germany) connected to membrane pumps (Thomas Division, Sheboygan, WI). The pumps withdrew air from the environment $(70 \mathrm{~L} / \mathrm{h})$ through a $3 \AA$ molecular sieve filter (Carl Roth $\mathrm{GmbH} \& \mathrm{Co} \mathrm{KG}$, Karlsruhe, Germany), the bag, and an adsorbent trap. The volatiles were trapped on charcoal (CLSA; Gränicher \& Quartero, Daumazan, France) for $60 \mathrm{~min}$ and TENAX ${ }^{\circledR}$ (TDS; Gerstel, Mühlheim, Germany) for $30 \mathrm{~min}$. In 2008, between August 29 and September 24, 29 samples were taken. During sample collection, the temperature was recorded with a HOBOC data logger (Onset Computer Corporation, Bourne, MA). The accumulative degree days (ADDs) were calculated by dividing the sum of the minimum and maximum temperature $\left({ }^{\circ} \mathrm{C}\right)$ per day by 2 and subtracting five (27). Table 1 shows the sampling regime, the start of sampling, and the average temperature between the sampling. Controls with both TENAX ${ }^{\circledR}$ and charcoal traps attached to empty plastic bags with filters were placed $20 \mathrm{~m}$ away from the decaying mice to collect the volatiles of the sampling site.

\section{Trace Analysis}

TDS Gas Chromatography-Mass Spectrometry (GC-MS)-The TDS tubes were thermally desorbed by a TDS thermodesorption system (Gerstel). The volatiles were focused in a KAS cryotrap (Gerstel) and separated on a $30 \mathrm{~m} \times 0.25 \mathrm{~mm}$ HP-5MS $(95 \%$ dimethyl-5\% diphenyl-polysiloxane) nonpolar column $(0.25 \mu \mathrm{L}$ film thickness) in a 6890N Network GC System; both systems were from Agilent Technologies (Santa Clara, CA). The carrier gas was helium with $99,999 \%$ purity and a gas flow of $1 \mathrm{~mL} / \mathrm{L}$. The oven program was set to an initial temperature of $40^{\circ} \mathrm{C}$ for $1.5 \mathrm{~min}$, which was then increased at a rate of $7.5^{\circ} \mathrm{C}$ per min to $200^{\circ} \mathrm{C}$ with a final hold of $5 \mathrm{~min}$. The 5973 quadrupole mass spectrometer (Agilent Technologies) was operated in the scan mode in the $\mathrm{m} / \mathrm{z}$ detection range of 20-354 per $0.5 \mathrm{sec}$. The resulting chromatograms were further evaluated using the Enhanced Chemstation version D00.00.38 software (Agilent Technologies), Mass Spectral Search Library of the National Institute of Standards and Technology (NIST, Gaithersburg, $\mathrm{MD}$ ), and MS Interpreter version 0.9f (NIST). Authentic standards were employed to confirm the identity of the compounds found in the samples (Table 2). Straight-chain hydrocarbons (C8 -C24) were used to calculate the linear retention index (28).

CLSA GC-MS-The CLSA tubes were eluted with $75 \mu \mathrm{L}$ of a dichloromethane/methanol (2:1) mixture. One microliter of the solution was injected through the injection port of a $6890 \mathrm{~N}$ Network GC System onto a $30 \mathrm{~m} \times 0.25 \mathrm{~mm}$ HP INNOWAX (poly(ethylenglycol)) polar column $(0.25 \mu \mathrm{m}$ film thickness); both systems were from Agilent Technologies. The carrier gas was helium with $99,999 \%$ purity and a gas flow of $1 \mathrm{~mL} / \mathrm{L}$. The oven program was set to an initial temperature of $50^{\circ} \mathrm{C}$ for $1.5 \mathrm{~min}$, which was then increased at a rate of $7.5^{\circ} \mathrm{C}$ per min to $250^{\circ} \mathrm{C}$ with a final hold of $5 \mathrm{~min}$. A 5973 quadrupole mass spectrometer (Agilent Technologies) was operated in the scan mode in the $\mathrm{m} / \mathrm{z}$ detection range of $20-354$ per $0.5 \mathrm{sec}$. The resulting chromatograms were further evaluated using the Enhanced Chemstation version D00.00.38 software, Mass Spectral Search Library of the National Institute of Standards and Technology, and MS Interpreter version 0.9f. Authentic standards were used to confirm the identity of the compounds in the samples (Table 2). Straight-chain hydrocarbons (C8-C24) were used to calculate the linear retention index (28). The identified compounds were quantitated from the peak areas of selected single ions in the CLSA eluate. Dimethyl disulfide was quantitated on the basis of $\mathrm{m} / \mathrm{z}=94$; undecane, $\mathrm{m} / \mathrm{z}=71$; heptanal, $\mathrm{m} /$ $\mathrm{z}=70 ; 3$-methylbutan-1-ol, $\mathrm{m} / \mathrm{z}=55$; octan-3-one, $\mathrm{m} / \mathrm{z}=99$; hexan-1-ol,

$\mathrm{m} / \mathrm{z}=56$; dimethyl trisulfide, $\mathrm{m} / \mathrm{z}=126$; nonanal, $\mathrm{m} / \mathrm{z}=57$; 1-octen-3-ol, $\mathrm{m} / \mathrm{z}=57$; phenol, $\mathrm{m} / \mathrm{z}=94$; and indole, $\mathrm{m} /$ $\mathrm{z}=117$. The median, minimum, and maximum of the compound concentrations are listed in Table 3. The samples were grouped into the following stages based on the optical impression of the stages of decay of the mice: fresh, bloated, active decay, and advanced decay (Table 4). The characteristics of each stage were presented by Dekeirsschieter et al. (12) and reviewed by Carter et al. (29). In few cases, the decaying mice showed characteristics of two stages, for instance, of the active stage and the advanced stage. The same effect was described by Matuszewski et al. (30), in relation to decaying pig carcasses. In these cases, the predominant stage was selected to evaluate the 
TABLE 1-Sampling regime of the 29 samples of the five mice, the average daily temperature, the minimum and maximum temperature, the stage of decay according to Table 4 and the accumulated degree days (ADDs).

\begin{tabular}{|c|c|c|c|c|}
\hline Date & $\begin{array}{c}\text { Average } \\
\text { daily } \\
\text { temperature } \\
\left({ }^{\circ} \mathrm{C}\right)\end{array}$ & $\begin{array}{l}\text { Minimum } \\
\text { daily } \\
\text { temperature } \\
\left({ }^{\circ} \mathrm{C}\right)\end{array}$ & $\begin{array}{l}\text { Maximum } \\
\text { daily } \\
\text { temperature } \\
\left({ }^{\circ} \mathrm{C}\right)\end{array}$ & $\begin{array}{c}\text { Mice (stage } \\
\text { of decay;ADD) }\end{array}$ \\
\hline $\begin{array}{l}29 \text { August } \\
2008\end{array}$ & 12 & 6 & 19 & 1 (fresh;0) \\
\hline $\begin{array}{l}30 \text { August } \\
2008\end{array}$ & 11 & 5 & 19 & 1 (fresh;40) \\
\hline $\begin{array}{l}31 \text { August } \\
2008\end{array}$ & 11 & 5 & 17 & 1 (bloated;53) \\
\hline $\begin{array}{l}1 \text { September } \\
2008\end{array}$ & 11 & 4 & 16 & \\
\hline $\begin{array}{l}2 \text { September } \\
2008\end{array}$ & 10 & 4 & 16 & $\begin{array}{l}1 \text { (active;76),2 } \\
\text { (fresh;0) }\end{array}$ \\
\hline $\begin{array}{l}3 \text { September } \\
2008\end{array}$ & 11 & 3 & 16 & $\begin{array}{c}1 \text { (active;85), } 2 \\
\quad \text { (bloated;20) }\end{array}$ \\
\hline $\begin{array}{l}4 \text { September } \\
2008\end{array}$ & 10 & 2 & 16 & \\
\hline $\begin{array}{l}5 \text { September } \\
2008\end{array}$ & 10 & 3 & 16 & $\begin{array}{l}1 \text { (advanced;103),2 } \\
\text { (active;38) }\end{array}$ \\
\hline $\begin{array}{l}6 \text { September } \\
2008\end{array}$ & 10 & 3 & 16 & \\
\hline $\begin{array}{l}7 \text { September } \\
2008\end{array}$ & 12 & 6 & 17 & $\begin{array}{l}1 \text { (advanced;124), } \\
2 \text { (advanced;60) }\end{array}$ \\
\hline $\begin{array}{l}8 \text { September } \\
2008\end{array}$ & 15 & 10 & 27 & \\
\hline $\begin{array}{l}9 \text { September } \\
2008\end{array}$ & 16 & 10 & 30 & 3 (fresh;0) \\
\hline $\begin{array}{l}10 \text { September } \\
2008\end{array}$ & 16 & 10 & 19 & $3($ fresh;22) \\
\hline $\begin{array}{l}11 \text { September } \\
2008\end{array}$ & 16 & 10 & 20 & 3 (bloated;35) \\
\hline $\begin{array}{l}12 \text { September } \\
2008\end{array}$ & 16 & 11 & 22 & $\begin{array}{l}3 \text { (active;47), } 4 \\
\text { (fresh;0) }\end{array}$ \\
\hline $\begin{array}{l}13 \text { September } \\
2008\end{array}$ & 16 & 11 & 21 & $\begin{array}{l}3 \text { (advanced;55), } 4 \\
\quad \text { (fresh;20) }\end{array}$ \\
\hline $\begin{array}{l}14 \text { September } \\
2008\end{array}$ & 16 & 11 & 21 & \\
\hline $\begin{array}{l}15 \text { September } \\
2008\end{array}$ & 15 & 11 & 20 & $\begin{array}{l}4 \text { (fresh;30), } 5 \\
\quad \text { (fresh;0) }\end{array}$ \\
\hline $\begin{array}{l}16 \text { September } \\
2008\end{array}$ & 14 & 11 & 20 & \\
\hline $\begin{array}{l}17 \text { September } \\
2008\end{array}$ & 13 & 10 & 19 & $\begin{array}{l}4 \text { (fresh;48), } 5 \\
\quad(\text { fresh;22) }\end{array}$ \\
\hline $\begin{array}{l}18 \text { September } \\
2008\end{array}$ & 13 & 9 & 19 & $\begin{array}{l}4 \text { (bloated;57), } 5 \\
\quad(\text { fresh;31) }\end{array}$ \\
\hline $\begin{array}{l}19 \text { September } \\
2008\end{array}$ & 13 & 8 & 19 & \\
\hline $\begin{array}{l}20 \text { September } \\
2008\end{array}$ & 12 & 8 & 19 & \\
\hline $\begin{array}{l}21 \text { September } \\
2008\end{array}$ & 12 & 7 & 19 & $\begin{array}{c}4 \text { (active;84), } 5 \\
\text { (bloated;59) }\end{array}$ \\
\hline $\begin{array}{l}22 \text { September } \\
2008\end{array}$ & 13 & 9 & 18 & $\begin{array}{l}4 \text { (advanced;90), } 5 \\
\text { (active;65) }\end{array}$ \\
\hline $\begin{array}{l}23 \text { September } \\
2008\end{array}$ & 10 & 2 & 15 & \\
\hline $\begin{array}{l}24 \text { September } \\
2008\end{array}$ & 18 & 11 & 30 & 5 (active;77) \\
\hline
\end{tabular}

correlation between the optical impression, ADDs, and selected compounds in the volatile profile (Fig. 1).

\section{Flies}

In the autumn of 2008, female $C$. vicina were caught from fresh ground meat (half pork, half beef) in the city of Göttingen. The species $C$. vicina was chosen as it is one of the first to arrive on a dead vertebrate and is present during all stages of decay, excluding the dry stage. This species is considered one of the most important in forensic entomology $(22,23)$ and is a model organism for the olfaction of blowflies $(20,25,26)$. Although Calliphora vomitoria is reported to be more abundant in forests of central Europe (31), it was decided nevertheless that C. vicina would be used. After removing the antennae for electrophysiological experiments, the development of the ovaries was assessed (32). This was used as an indicator of the predilection of the flies to oviposit and therefore exhibits high electrophysiological performance. Only flies with developed ovaries were selected for the electrophysiological measurements.

\section{Electroantennogram}

All compounds identified in the CLSA samples were tested in paraffin oil (Uvasol quality; Merck/VWR, Darmstadt, Germany) dilutions from $10^{-9}$ to $10^{-2} \mathrm{mg} / \mathrm{mg}$. For each dilution step, a $2 \mathrm{~cm}^{2}$ filter paper was drenched with $200 \mu \mathrm{L}$ of the dilution. The filter paper was inserted into a $10-\mathrm{mL}$ glass syringe, and $2.5 \mathrm{~mL}$ of the air was blown into a stream of humidified air $\left(500 \mathrm{~mL} / \mathrm{min}, 23^{\circ} \mathrm{C}, 80 \%\right.$ relative humidity), which was passed over the excised antenna of female $C$. vicina. For each dilution, three antennae were used, and every antenna was blown three times. The depolarization of the antennae was monitored by an electroantennographic (EAG) setup (Professor Koch, Kaiserslautern, Germany) consisting of a preamplifier, main amplifier, frequency filter, and adjustment amplifier The signals were analyzed by the GC Chemstation software. (for more details, see Weissbecker et al. [33]). Significant differences in responses to the negative paraffin oil control on filter paper and the compound dilution were calculated by the $U$-test or $t$-test.

\section{Results \\ Decaying Process}

Table 4 shows the four different stages of decay according to the description given by Dekeirsschieter et al. (12). This approach was used to group the volatile samples. $C$. vicina is present during all of these stages $(22,23)$. In Table 1 , the sampling regime, the average daily temperature, the stage of decay, and the ADDs are shown. During the experiment, the average day temperature varied between $10^{\circ} \mathrm{C}$ and $18^{\circ} \mathrm{C}$. While the first three mice entered the bloated (Fig. 3) or active stage (Fig. 4) after one or 2 days, mouse four entered the bloated stage after 6 days and mouse five after 4 days.

The ADD was calculated to compare the temperature dependent processes of decay. However, the values of the ADDs were not correlated to the stages of decay in this experiment. The fresh stage (Fig. 2) shows a maximum ADD of 48 , while the bloated stage shows a minimum ADD of 35 . The ADD range of the active stage of decay $\left(\mathrm{ADD}_{\min }=20 ; \mathrm{ADD}_{\max }=85\right)$ overlaps with all other stages, whereas only the advanced stage (Fig. 5) $\left(\mathrm{ADD}_{\min }=55 ; \mathrm{ADD}_{\max }=124\right)$ has a constantly higher $\mathrm{ADD}$ value than the fresh stage.

In this experiment, insects were present during all four stages of decay, most of them being members of the order Diptera or Coleoptera. The predominant fly families were Calliphoridae (C. vicina, C. vomitoria) and Dryomyzidae. Calliphoridae were present throughout the decaying process and oviposited occasionally, whereas Dryomycidae preferred the fresh and bloated stages for feeding. A greater number of flies visited the remains during the decay of mouse one and two 
TABLE 2-Compounds identified by both analytical methods, in the order of their linear retention index on the polar column.

\begin{tabular}{|c|c|c|c|c|c|c|}
\hline No. & Compound & $\begin{array}{c}\text { CLSA } \\
\text { (LRI) } \\
\text { INNOWAX }\end{array}$ & $\begin{array}{c}\text { TDS } \\
\text { (LRI) } \\
\text { HP-5MS }\end{array}$ & $\begin{array}{c}\text { Chemical } \\
\text { Classes }\end{array}$ & $\begin{array}{l}\text { Authentic Standards } \\
\text { (Company, Purity, } \\
\text { CAS No.) }\end{array}$ & Literature \\
\hline 1 & Dimethyl disulfide & 1083 & 755 & Sulfur compound & Merck, 98\%, 624-92-0 & $(9,12-20)$ \\
\hline 2 & Undecane & 1100 & 1100 & Alkane & Acros, 99\%, 1120-21-4 & $(13,14)$ \\
\hline 3 & Heptanal & 1181 & 906 & Aldehyde & Acros, $95 \%, 111-71-7$ & $(9,12,13,17,18,42)$ \\
\hline 4 & 3-Methylbutan-1-ol & 1195 & 759 & Alcohol & Aldrich, $99,5 \%, 123-51-3$ & $(1,8,12,47)$ \\
\hline 5 & Octan-3-one & 1251 & 992 & Ketone & VWR, 96\%, 106-68-3 & $(8,58)$ \\
\hline 6 & Hexan-1-ol & 1341 & 884 & Alcohol & Aldrich, 98\%, 111-27-3 & $(9,16,17,25,42)$ \\
\hline 7 & Dimethyl trisulfide & 1386 & 976 & Sulfur compound & SAFC, 98\%, 3658-80-8 & $(9,12-16,19,20)$ \\
\hline 8 & Nonanal & 1392 & 1113 & Aldehyde & Merck, 98\%, 124-19-6 & $(8,13,14,17,42)$ \\
\hline 9 & 1-Octen-3-ol & 1440 & 985 & Alcohol & Merck, 98\%, 3391-86-4 & $(8,17,48,57)$ \\
\hline 10 & Phenol & 1980 & 1021 & Aromatic & Aldrich, $99 \%, 108-95-2$ & $(12,13,15,17,68)$ \\
\hline 11 & Indole & 2442 & 1331 & Aromatic heterocycle & Fluka, 99\%, 120-72-9 & $(1,12,16-19,34)$ \\
\hline
\end{tabular}

LRI, linear retention index; CAS, Chemical Abstracts Service.

than during the decay of mouse three, four, and five. Oviposition occurred only on mouse one and two. These discrepancies can possibly be explained by shorter daylight hours, which decreased from $14 \mathrm{~h}$ during the decay of mouse one and two, to $12 \mathrm{~h}$ during the decay of the other mice. The prevalent beetle species observed were Geotrupes spp., Geotrupidae and Nicrophorus spp., Silphidae (Nicrophorus vespilloides, Nicrophorus vespillo).

\section{Trace Analysis}

Eleven compounds were identified by both methods adopted in the experiment. The compounds are listed in the order of their retention index on the polar column (CLSA) and are assigned to

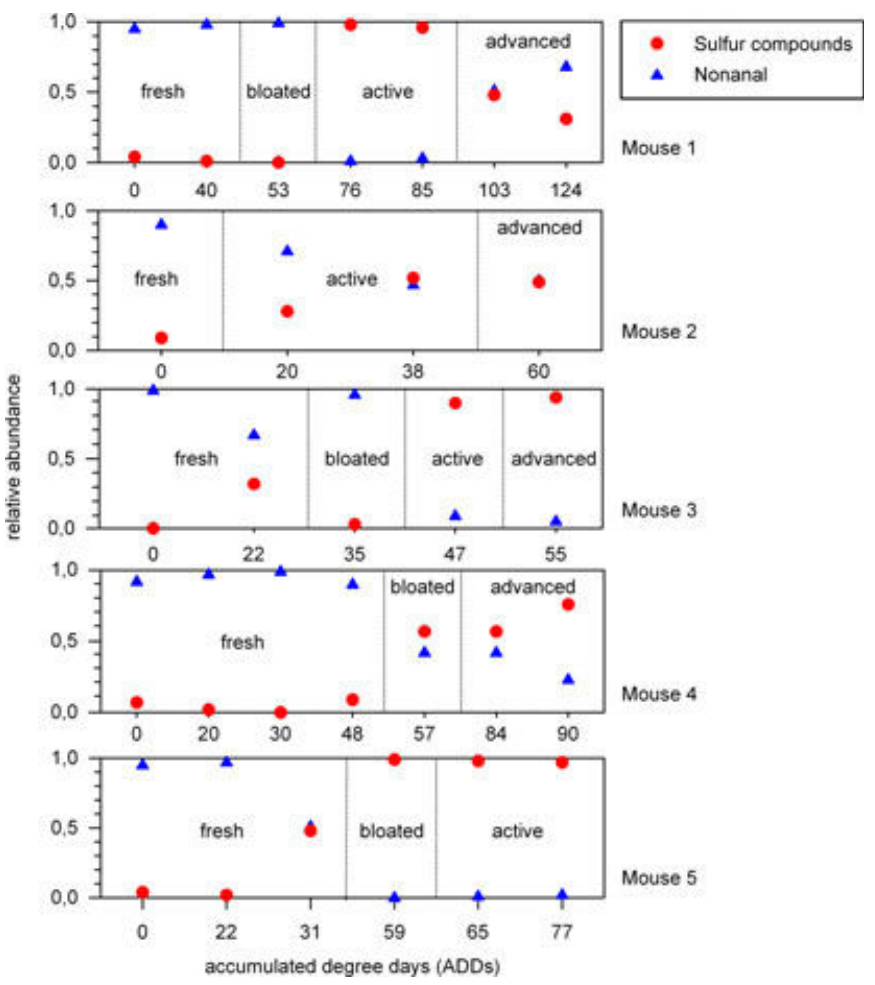

FIG. 1-Relative emission patterns of nonanal and the sulfur compounds dimethyl disulfide and dimethyl trisulfide across the four stages of decay in the five mice. On the $\mathrm{x}$-axis the $A D D$ value of each sample is given and the $\mathrm{y}$-axis shows the relative abundance of nonanal and the sulfur compounds. the following chemical classes: alcohols, ketones, alkanes, aldehydes, sulfur compounds, aromatic compounds, and aromatic heterocycles (Table 2). The quantitative data are presented in Table 3. Both sulfur compounds were predominantly found in the active stage of decay (dimethyl trisulfide, $<1-104 \mathrm{ng} / \mu \mathrm{L}$; dimethyl disulfide, $<1-60 \mathrm{ng} / \mu \mathrm{L})$, as well as 3-methylbutan-1-ol $(1-20 \mathrm{ng} / \mu \mathrm{l})$ and phenol $(<1-7 \mathrm{ng} / \mu \mathrm{L})$. Nonanal was found in high concentrations during the fresh stage of decay $(<1-52 \mathrm{ng} /$ $\mu \mathrm{L})$ and in lower concentrations during the other stages $(<1-12$ $\mathrm{ng} / \mu \mathrm{L})$. Hexan-1-ol, 1-octen-3-ol, heptanal, and indole were found in very low quantities throughout the decaying process $(<1-1 \mathrm{ng} / \mu \mathrm{L})$. Undecane was present in higher concentrations in samples throughout the entire experiment $(2-5 \mathrm{ng} / \mu \mathrm{L})$, with the exception of one sample in the fresh stage of decay with low concentration of undecane.

\section{Electrophysiology}

The results of the EAG experiments are presented in Table 5. Significant differences between the paraffin control and the stimulus were observed with dimethyl trisulfide at a dilution of $10^{-9}$ and dimethyl disulfide at a dilution of $10^{-8}$. Nonanal, hexan-1-ol, 1-octen-3-ol, 3-methyl-butan-1-ol, and heptanal were detected at dilutions of $10^{-5}$ or $10^{-4}$. Octan-3-one, indole, and phenol stimulated the antennae at higher concentrations, and undecane did not stimulate the antennae at all.

\section{Discussion}

The idea of distinct stages of vertebrate decay is used by several authors (for review, see [29]). Clearly, this approach is always subjective (29), as the process of decay is continuous. The stage model ignores the fact that a carcass can show the characteristics of two stages of decay at the same time (30). Matuszewski et al. (30) discuss processes-oriented approach and describe the decaying process using the onset, duration, and rate of the decay characteristics. It is most likely that the mosaic effect, discussed by Matuszewski et al. (30), influences the volatile profile, particularly of larger vertebrates. This could be problematic for volatile-based PMI estimation, as the quantity of the emitted volatiles would depend on several overlapping processes. The volatile profile of a small cadaver should not show the influence of the mosaic effect, because the decaying process is much faster. Therefore, an inhomogeneous decay would not affect the volatile quantities to the same extent as in larger vertebrates. 


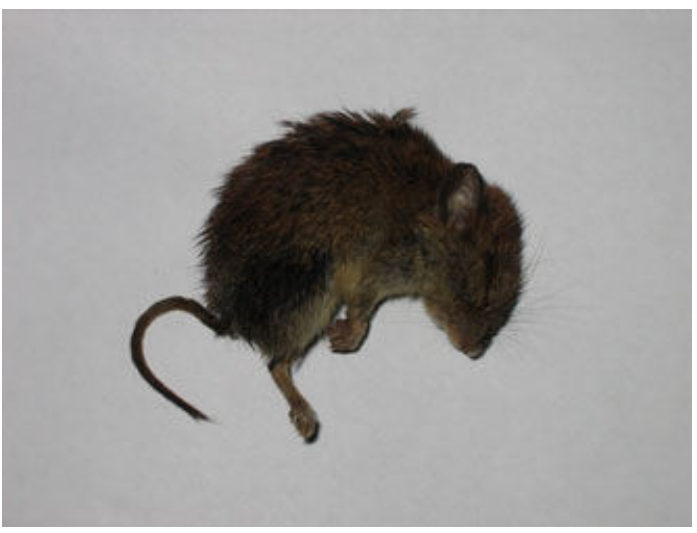

FIG. 2-Fresh stage of decay of mouse one (29 August 2011).

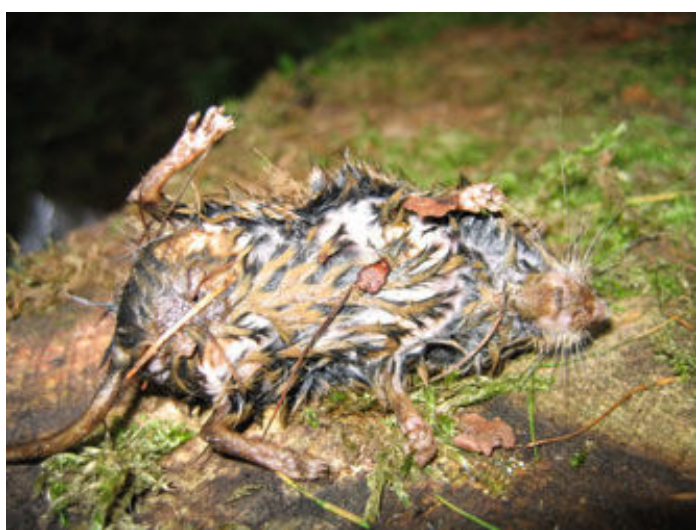

FIG. 3-Bloated stage of decay of mouse two (3 September 2011).

Temperature is another important aspect to be considered. The process of decay is mainly driven by microbial metabolism and insect feeding, and both factors are strongly influenced by the temperature regime. Calculating the ADDs for PMI determination was inspired by entomological studies and evaluated for forensic science $(27,34)$ to compare decaying processes. A linear model seems to be an inappropriate approach, because the temperature has a nonlinear influence on enzymatic activity (5), as well as on the vapor pressure of volatile compounds (35). Vass et al. (34) attempted to solve this problem by calculating the cumulative degree hours for every $12 \mathrm{~h}$, thereby separating day and night temperatures. However, they state that their results still lack any strong correlation between temperature and rate of decay in graves, indicating the influence of other factors like humidity, oxygen availability, or soil composition (34). Michaud and Moreau calculated a nonlinear regression model based on four ADDs, which explained the decay of the 12 exposed pig carcasses in their study (27).

In the experiment conducted in Göttingen, only the fresh and advanced stages of the decaying mice showed no overlapping ADD values. This confirms the limitations of the stages approach and the linear model for rapid decaying processes. In general, describing the decomposition in distinct stages is problematic; however, insect succession on a cadaver is partly correlated to the process of decomposition $(22,23,31)$. This correlation might occur, because certain insect species detect core volatiles, which are always present at a certain stage of the decaying process. The electrophysiological studies in the experiment show dimethyl

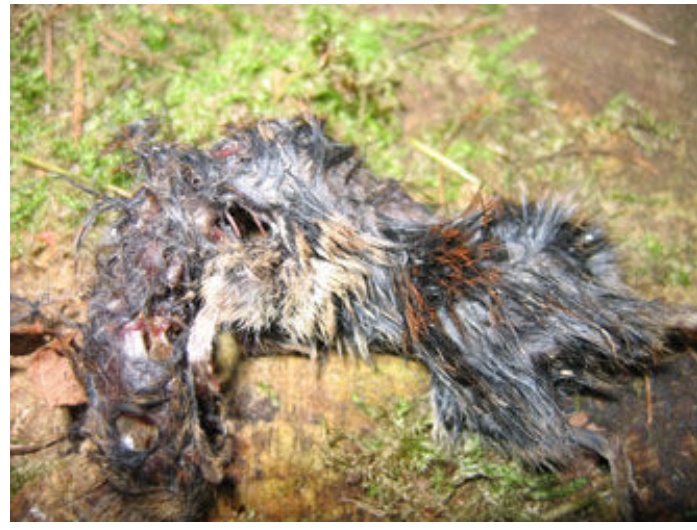

FIG. 4-Active stage of decay of mouse one (2 September 2011).

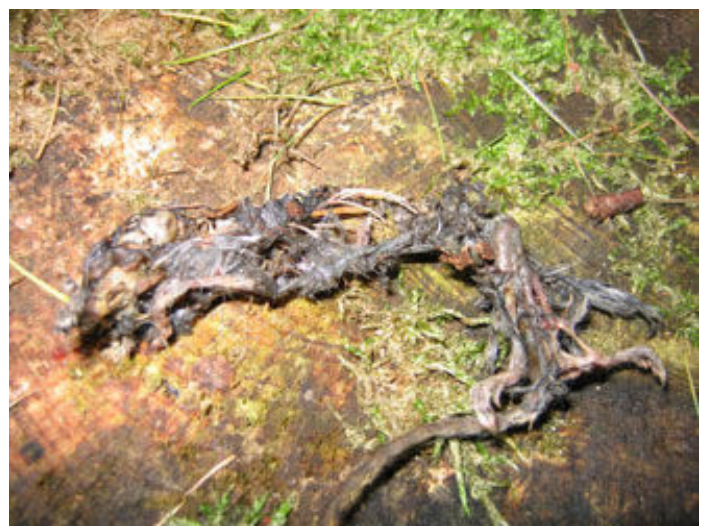

FIG. 5-Advanced stage of decay of mouse one (5 September 2011).

disulfide and dimethyl trisulfide were detected at very low concentrations of $10^{-9}$ and $10^{-8}$ by female blowfly antennae (Table 5). Figure 6 shows the dose-response curve for female C. vicina antennae with a calculated $\mathrm{ED}_{10}$ of $6 \times 10^{-10}$ (36). The curve shows biphasic development, which is typical for two olfactory receptor neuron populations of different sensitivities. Furthermore, the formation of dimethyl disulfide and dimethyl trisulfide has been reported in most experimental literature on the decomposition of vertebrates $(9,12-17,20)$. The cited literature, as well as the trace analysis and electrophysiology presented in this study, lead to the conclusion that both compounds are important cues for the process of decay and potential core volatiles. In general, sulfur compounds are formed by the microbial degradation of methionine and cysteine, which are two sulfur-containing amino acids in the body $(1,19,37,38)$. Among the sulfur compounds formed, methanethiol is emitted by the following bacteria: Hafnia alvei, Enterobacter agglomeran, Serratia liquefaciens, Alteromonas putrefaciens, and Aeromonas hydrophila, which grow on vertebrate tissue $(39,40)$. The oxidative dimerization of methanethiol contributes to the formation of dimethyl disulfide and dimethyl trisulfide both in vivo and in vitro (41). Furthermore, the bacteria belonging to the Thiobacillus group can transform sulfur compounds under aerobic conditions and might therefore catalyze this reaction (19).

The high sensitivity of female C. vicina toward sulfur compounds is important for tracing cadavers, which was demonstrated in behavioral tests (21). However, it is unlikely that sulfur compounds play a role in initial cadaver detection, because the biochemical processes described here do not yield 
TABLE 3-Emission rates of the volatile compounds quantitated in the CLSA samples in ng/ $\mu$. The median displays the average concentration in all samples per stage. The $\mathrm{n}$ value shows the number of samples analyzed in each stage of decay according to Table 4. Min and Max show the minimum and maximum concentration of the volatile compounds in the samples. If no value is given for the minimum, the compound was absent in at least one sample. Less than one indicates the presence of the compound below the threshold for quantitation in all samples.

\begin{tabular}{|c|c|c|c|c|c|c|c|c|c|c|c|c|}
\hline \multirow[b]{2}{*}{ Compound } & \multicolumn{3}{|c|}{ Fresh, $n=12$} & \multicolumn{3}{|c|}{ Bloated, $n=3$} & \multicolumn{3}{|c|}{ Active, $n=8$} & \multicolumn{3}{|c|}{ Advanced, $n=6$} \\
\hline & Median & Min & Max & Median & Min & Max & Median & Min & Max & Median & Min & Max \\
\hline Dimethyl trisulfide & 0 & - & $<1$ & $<1$ & 0 & 1 & 17 & $<1$ & 104 & $<1$ & - & 2 \\
\hline Dimethyl disulfide & $<1$ & - & 1 & 1 & $<1$ & 2 & 11 & $<1$ & 60 & 2 & $<1$ & 10 \\
\hline Nonanal & 2 & $<1$ & 52 & 2 & 2 & 2 & 2 & $<1$ & 12 & 1 & $<1$ & 3 \\
\hline Hexan-1-ol & $<1$ & - & 1 & $<1$ & $<1$ & 1 & $<1$ & - & 1 & $<1$ & $<1$ & 1 \\
\hline 1-Octen-3-ol & $<1$ & $<1$ & $<1$ & $<1$ & $<1$ & $<1$ & $<1$ & $<1$ & $<1$ & $<1$ & $<1$ & $<1$ \\
\hline 3-Methylbutan-1-ol & $<1$ & $<1$ & 1 & 1 & $<1$ & 1 & 5 & 1 & 20 & 2 & $<1$ & 3 \\
\hline Heptanal & $<1$ & - & 1 & $<1$ & $<1$ & $<1$ & $<1$ & - & $<1$ & $<1$ & $<1$ & $<1$ \\
\hline Octan-3-one & $<1$ & - & 2 & $<1$ & - & $<1$ & $<1$ & - & 1 & $<1$ & 0 & $<1$ \\
\hline Indole & $<1$ & - & $<1$ & $<1$ & - & $<1$ & $<1$ & - & 1 & $<1$ & - & $<1$ \\
\hline Phenol & $<1$ & - & $<1$ & $<1$ & $<1$ & $<1$ & 2 & $<1$ & 7 & $<1$ & $<1$ & $<1$ \\
\hline Undecane & 3 & - & 4 & 3 & 2 & 3 & 2 & 2 & 4 & 2 & 2 & 5 \\
\hline
\end{tabular}

TABLE 4-Four stages of the decomposition process of the mice during the sampling period.

\begin{tabular}{|c|c|c|c|}
\hline $\begin{array}{l}\text { Stage of } \\
\text { Mice Decay }\end{array}$ & $\begin{array}{l}\text { Length } \\
\text { of Time } \\
\text { (Days) }\end{array}$ & Characteristics & Pictures \\
\hline Fresh & $1-4$ & $\begin{array}{l}\text { Rigor mortis; lowered } \\
\text { body temperature }\end{array}$ & $\begin{array}{l}\text { Figure } 2 \text { shows } \\
\text { the fresh stage } \\
\text { of decay of } \\
\text { mouse one } \\
\text { (29 August 2011) }\end{array}$ \\
\hline Bloated & 1 & $\begin{array}{l}\text { Putrefaction inside } \\
\text { the mice causes } \\
\text { bloating; loss of } \\
\text { hair begins }\end{array}$ & $\begin{array}{l}\text { Figure } 3 \text { shows } \\
\text { the bloated stage } \\
\text { of decay of } \\
\text { mouse two } \\
\text { (3 September 2011) }\end{array}$ \\
\hline $\begin{array}{l}\text { Active } \\
\text { decay }\end{array}$ & $1-3$ & $\begin{array}{l}\text { Cadaver inflates and } \\
\text { microbial degradation } \\
\text { of inner tissues } \\
\text { increases }\end{array}$ & $\begin{array}{l}\text { Figure } 4 \text { shows the } \\
\text { active stage of } \\
\text { decay of mouse one } \\
\text { (2 September } \\
\text { 2011) }\end{array}$ \\
\hline $\begin{array}{c}\text { Advanced } \\
\text { decay }\end{array}$ & $1-3$ & $\begin{array}{l}\text { Most of the soft tissues } \\
\text { are assimilated by } \\
\text { microbial activity; only } \\
\text { hair, bones, cartilage, } \\
\text { and skin are left }\end{array}$ & $\begin{array}{l}\text { Figure } 5 \text { shows the } \\
\text { advanced stage } \\
\text { of decay of } \\
\text { mouse one } \\
\text { (5 September 2011) }\end{array}$ \\
\hline
\end{tabular}

sulfur compounds immediately after death. Different volatiles or a combination of factors could be involved in initial cadaver detection. The aldehydes nonanal and heptanal are electrophysiologically active at dilutions of $10^{-5}$ or $10^{-4}$. Among the volatiles in Table 3, nonanal is the only compound present in high concentrations at the onset of decay. Therefore, nonanal could be a cue for the detection of dead vertebrates shortly after death. In general, aldehydes are important in the decay process of vertebrates. Nonanal and heptanal have been identified by several authors $(8,9,12-14,17,18,42)$. Degradation of fat on the skin and fur of the mice might lead to the formation of nonanal in the beginning of the decaying process. It is possible that the same processes that yield nonanal on the living skin (42) also contribute to its formation in the fresh stage of decay. As $C$. vicina is reported to be abundant on cadavers within minutes after death, flies may follow additional indications, for example, the immobility of the cadaver or carbon dioxide concentration. After the onset of decay, the degradation of depot fat or intracellular fat might contribute to the formation of nonanal. Dekeirsschieter et al. (12) mentioned an increase in aldehyde formation during the later stages of decay, and Vass et al. (13) identified aldehydes in high relative concentrations in bones. As fat is a component of marrow that persists even in ancient bones (43-46), the increasing amount of aldehydes in the volatile pattern of decaying vertebrates could be derived from lipid oxidation in bones (47). Unfortunately, there is a lack of detailed studies on biogenic aldehyde formation arising from fatty acid degradation $(48,49)$. Although the beta-oxidation enzymes in prokaryotes, fungi, and plants differ from each other $(49,50)$, their catabolic activity is comparable and yields acetyl-CoA or propionyl-CoA (51). This indicates that aldehydes are not typically emitted from cadavers, but from any source in which lipids are oxidized, and that the emission of nonanal alone is too unspecific for tracing a cadaver in any stage of decay. Therefore, volatile-based PMI estimation or cadaver dog training should include more than one volatile.

Trace analytical and electrophysiological results from the experiment indicate that nonanal and the sulfur compounds are core volatile candidates. The first and second requirements for core volatiles are fulfilled, because both nonanal or the sulfur compounds are present during the whole process of decay and are emitted in high quantities (Table 3). To evaluate whether the volatiles show a constant quantitative emission pattern, the relative quantities of the compounds were displayed. Temperature variation, variation in the adsorption performance of the charcoal tubes, and slightly different suction rates of the pumps can influence the quantity of the volatiles in the samples. Therefore, within one sample, the sum of nonanal, dimethyl disulfide, and dimethyl trisulfide was regarded as $100 \%$. The absolute quantities of both nonanal and the sulfur compounds were expressed as the percentage $(0.0-1.0)$ of this sum. As an example, if $10 \mathrm{ng} / \mu \mathrm{l}$ nonanal, $20 \mathrm{ng} / \mu \mathrm{L}$ dimethyl disulfide, and $20 \mathrm{ng} / \mu \mathrm{L}$ dimethyl trisulfide are found in one sample, the sum is $50 \mathrm{ng} / \mu \mathrm{L}$. In this case, the relative amount of nonanal in this sample is $10 \mathrm{ng} / \mu \mathrm{L} / 50 \mathrm{ng} / \mu \mathrm{L}=0.2$, and the relative amount of the sulfur compounds in this sample is $(20 \mathrm{ng} / \mu \mathrm{L}+20 \mathrm{ng} / \mu \mathrm{L}) / 50 \mathrm{ng} /$ $\mu \mathrm{L}=0.8$. Assuming that temperature and suction rate influence the emissions of the three compounds to the same extent, the relative quantities of all samples are compared in Fig. 1. There is a constant relation between nonanal and the sulfur compounds during the process of decay. In the early stages of decomposition, nonanal dominates, whereas in the later stages, this relation changes and the sulfur compounds are predominant. 


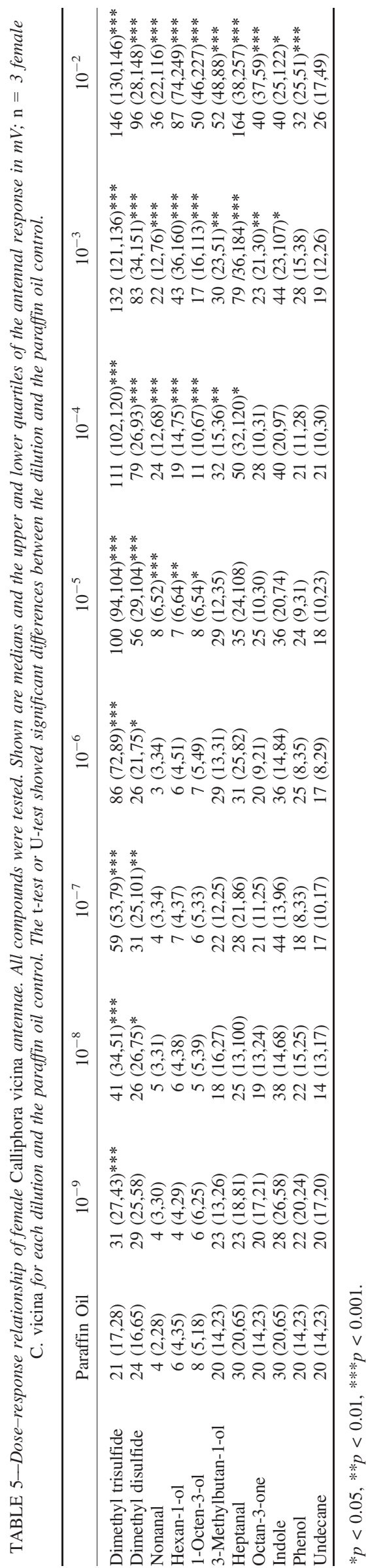

During the decay of mouse one and mouse three, this relation changed abruptly, whereas mouse two, four, and five show a slower change in the relation. Abrupt temperature changes did not occur (Table 4); therefore, different factors influenced the relation between nonanal and the sulfur compounds. One important factor could be the condition of the intestine during the early stages of decay. An intact intestine will lead to an accumulation of volatiles inside the cadaver. In this case, the cadaver would bloat until the skin breaks and the volatiles leave the body, changing the volatile profile abruptly. If there are holes in the intestine because of insect feeding or physical injury, the release of decompositional volatiles will occur earlier and in slowly increasing quantities. The mosaic effect might also influence the volatile emission in the early stages of decay. Most probably, the process of bloating cannot be regarded as the bloating of the intestine as a whole. While gases accumulate in intact regions of the intestine, they might leave through injuries in other parts. This could be important, especially where larger vertebrates are concerned.

The emission pattern presented in Fig. 1 shows a constant development of the relation between nonanal and the sulfur compounds for each mouse. However, the changes in the volatile pattern cannot be correlated accurately to the stages of decay. In the fresh stage of the decay (Fig. 2), nonanal clearly dominates the volatile profile. The only exception is the third sample of mouse five, where nonanal and the sulfur compounds are emitted in equal concentration. The volatile composition in the bloated stage (Fig. 3) does not show such a consistency. Mouse one and three show a high concentration of nonanal, whereas mouse five shows a high concentration of the sulfur compounds. Mouse four shows an almost equal concentration of both. In the active stage, the sulfur compounds are predominant in the volatile profile. Exceptions are the first sample of mouse two (ADD 20), where the concentration of nonanal is high, and the second sample in the active stage (Fig. 4) of mouse two where the concentrations of nonanal and the sulfur compounds are equal. In the advanced stage (Fig. 5), the compounds are either emitted in the same relative quantities (mouse one, ADD 103; mouse two and mouse four, ADD 84), or the sulfur compounds are emitted in relatively higher quantities (mouse three and mouse four, ADD 90). In the sample with the highest ADD, nonanal dominates (mouse one, ADD 124). An increasing amount of aldehydes in the later stages of decay has already been reported by Dekeirsschieter et al. (12) and might be connected to lipid oxidation in bones $(13,43-46)$.

The calculated ADD values do not sufficiently explain the volatile emission. A nonlinear model might show a stronger correlation. In this study, the dominance of the nonanal emission lasts to an ADD range between ADD 20 and ADD 53. The ADD range in which the relation between nonanal and the sulfur compounds changes begins at ADD 31 and ends at ADD 84 , while the ADD values of the sulfur compound dominance range between ADD 47 and ADD 90.

Neither the stages approach nor the linear temperature model can explain the volatile emission in this experiment. New models have to be developed to describe the process of decay to evaluate the hypothesis that the PMI can be estimated by means of volatile analysis. Temperature-based models might lack availability of data, as it has been found that temperature data provided by weather stations are not always sufficient for PMI estimation (52). More quantitative data on volatile emissions must be collected. Additional core volatiles emitted by larger vertebrates could increase the correlation of the volatile 


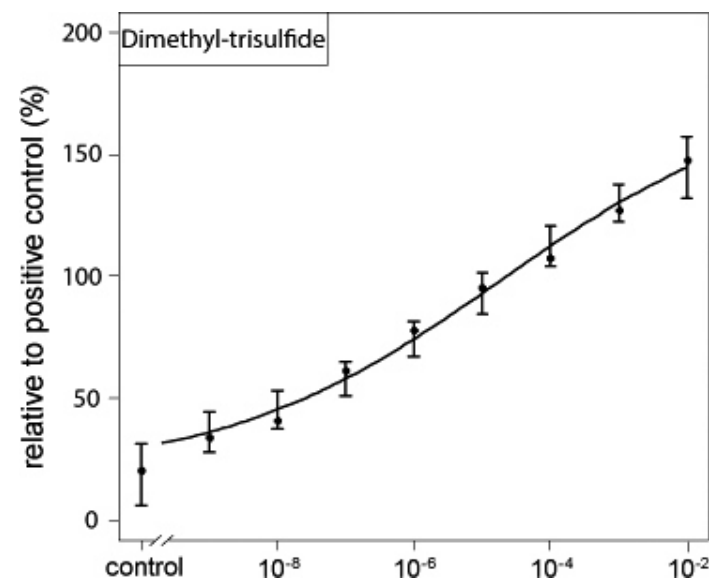

FIG. 6-Biphasic development of the dose-response reaction of female Calliphora vicina antennae toward dimethyl trisulfide.

emission during the decaying process. Investigation into the olfaction of other carrion visitors would be interesting, especially those species that appear during the early stages of decay, and may detect important core volatiles (31).

The higher fusel alcohol 3-methylbutan-1-ol is a candidate for an additional core volatile in the model presented in Fig. 1. Female blowflies can detect this compound at a dilution of $10^{-4}$ (Table 5), but in this experiment, the compound showed a high quantitative variation between the samples. 3-Methylbutan-1-ol is a byproduct of L-leucine degradation by the Ehrlich pathway (53). It is also a byproduct of the anabolic biosynthetic pathways in microbes $(47,54,55)$. Dekeirsschieter et al. (12) identified the compound in the headspace of decaying pig, and Ercolini et al. (8) detected the compound in the headspace of tainted meat. Moraxella phenylpyruvica, Staphylococcus xylosus, S. starnosus transforme, Saccharomyces cerevisiae, and Candida utilis degrade leucine to 3-methylbutan-1-ol $(53,54,56)$. The experiments performed in Göttingen showed that female blowflies could detect hexan-1-ol and 1-octen-3-ol at dilutions of $10^{-5}$ (Table 5). Both compounds showed very low absolute and relative emission in this experiment (Table 3), which discriminates them as core volatiles. However, human remains might emit the compounds in higher quantities. The eight-carbon volatile compound 1-octen-3-ol is formed during lipid oxidation of compounds such as linoleic acid (48) and arachidonic acid (57). The compound is predominantly emitted by fungal activity (58-63) and is used as the main fungal aroma compound in the food industry (64). 1-Octen-3-ol forms during the degradation of muscle tissue $(8,17)$ human blood, bone, and fat $(17)$; and the skin of fish (57). Haze et al. (42) identified hexan-1-ol in the headspace of living human bodies, while Hoffman et al. (17) identified this compound in the headspace of decaying human blood, testicle, muscle, fat, and bone. The compound is also emitted by tainted meat (9) and human cadavers (16). Fungal metabolism (58-63) and lipid oxidation in plants (65) also yield hexan-1-ol. The detection of 1-octen-3-ol and hexan-1-ol by $C$. vicina antennae (Table 5) could be useful for tracking the uptake of nutrition from flowers (25) and fungi (58), but is not necessarily important when tracing a cadaver.

Octan-3-one, indole, phenol, and undecane are, essentially, insignificant compounds to female blowflies, and among these volatiles, only undecane shows slightly higher concentrations in the volatile samples of decaying mice (Table 3). This discriminates them as core volatiles, although this might be different for larger vertebrates. Octan-3-one is the second eight-carbon volatile identified in this experiment. It has been identified in the headspace of tainted meat (8) and is prominent in the headspace of fungi (58). Lipid oxidation in fungi yields octan-3-one (48). Indole, a degradation product of the amino acid tryptophan (66), is found to be emitted from decaying pig (12); human blood, testicles, and adipoceres (17); and decaying humans (16,34). The volatile compound phenol was identified in the headspace of decaying pig (12) and decaying human remains $(13,15)$ and is derived from the bacterial metabolism of tyrosine in organisms such as the human intestine $(67,68)$. Undecane was detected in the headspace of human bones and at human burial sites $(13,14)$. Further information on postmortem volatiles of vertebrate tissue can be found in Paczkowski and Schütz (69).

\section{Conclusions}

The pattern of volatiles emitted from decaying vertebrates is complex. The olfactory perception of $C$. vicina and other carrion visiting invertebrates can help in the selection of core volatiles. Among the detected substances, sulfur compounds are the most important cues for tracing deceased vertebrates. Examination of the relative amounts of sulfur compounds and nonanal facilitates differentiation within the decaying process showed that the relative amounts are correlated neither to the stages of decay nor to the calculated ADD. This information might be a step toward establishing a volatile-based postmortem estimation method with potential forensic application.

\section{Acknowledgments}

We would like to thank Sara Nicke for supporting the electrophysiological experiments and statistical analysis of Table 5, Maximilian v. Fragstein und Niemsdorff for the statistical analysis of Fig. 6, and our reviewers for their helpful comments during the revision of the manuscript.

\section{References}

1. Gill-King H. Chemical and ultrastructural aspects of decomposition. In: Haglund WD, Sorg MH, editors. Forensic taphonomy, the postmortem fate of human remains. Boca Raton, FL: CRC Press, 1997;93 -108 .

2. Saukko P, Knight B. Knight's forensic pathology, 3rd rev edn. London: Edward Arnold, 2004.

3. Ito $\mathrm{T}$, Ando $\mathrm{T}$, Mayahara $\mathrm{H}$, Miyajima $\mathrm{H}$, Ogawa $\mathrm{K}$. Postmortem changes in the rat-kidney. 1. Histopathological, electron-microscopic, and enzyme histochemical-studies of postmortem changes at room-temperature. Acta Histochem Cytochem 1991;24:135-51.

4. Ito $\mathrm{T}$, Ando $\mathrm{T}$, Mayahara $\mathrm{H}$, Miyajima H, Ogawa K. Postmortem changes in the rat-kidney. 2. Histopathological, electron-microscopic, and enzyme histochemical-studies of postmortem changes at 0-degrees-C. Acta Histochem Cytochem 1991;24:153-66.

5. Goll DE, Otsuka Y, Nagainis PA, Shannon JD, Sathe SK, Muguruma M. Role of muscle proteinases in maintenance of muscle integrity and mass. J Food Biochem 1983;7:137-77.

6. Koohmaraie $\mathrm{M}$. The role of $\mathrm{Ca}-2+$-dependent proteases (calpains) in postmortem proteolysis and meat tenderness. Biochimie 1992;74:239-45.

7. Dransfield E. Modeling postmortem tenderization 3. Role of calpain-I in conditioning. Meat Sci 1992;31:85-94.

8. Ercolini D, Russo F, Nasi A, Ferranti P, Villani F. Mesophilic and psychrotrophic bacteria from meat and their spoilage potential in vitro and in beef. Appl Environ Microbiol 2009;75:1990-2001.

9. Stutz HK, Silverman GJ, Angelini P, Levin RE. Bacteria and volatile compounds associated with ground-beef spoilage. J Food Sci 1991;56:1147-53.

10. Moore WEC, Holdeman LV. Human fecal flora-normal flora of 20 Japanese-Hawaiians. Appl Microbiol 1974;27:961-79. 
11. Gray TRG, Williams ST. Soil microorganisms. Edinburgh: Oliver and Boyd, 1971.

12. Dekeirsschieter J, Verheggen FJ, Gohy M, Hubrecht F, Bourguignon L, Lognay G, et al. Cadaveric volatile organic compounds released by decaying pig carcasses (Sus domesticus L.) in different biotopes. Forensic Sci Int 2009;189:46-53.

13. Vass AA, Smith RR, Thompson CV, Burnett MN, Dulgerian $\mathrm{N}$ Eckenrode BA. Odor analysis of decomposing buried human remains. J Forensic Sci 2008;53:384-91.

14. Vass AA, Smith RR, Thompson CV, Burnett MN, Wolf DA, Synstelien JA, et al. Decompositional odor analysis database. J Forensic Sci 2004;49:760-9.

15. Statheropoulos M, Agapiou A, Spiliopouiou C, Pallis GC, Sianos E. Environmental aspects of VOCs evolved in the early stages of human decomposition. Sci Total Environ 2007;385:221-7.

16. Statheropoulos M, Spiliopouiou C, Agapiou A. A study of volatile organic compounds evolved from the decaying human body. Forensic Sci Int 2005; 153:147-55.

17. Hoffman EM, Curran AM, Dulgerian N, Stockham RA, Eckenrode BA. Characterization of the volatile organic compounds present in the headspace of decomposing human remains. Forensic Sci Int 2009;186:6-13.

18. Lorenzo N, Wan TL, Harper RJ, Hsu YL, Chow M, Rose S, et al. Laboratory and field experiments used to identify Canis lupus var. familiaris active odor signature chemicals from drugs, explosives, and humans. Analyt Bioanalyt Chem 2003;376:1212-24.

19. Dent BB, Forbes SL, Stuart BH. Review of human decomposition processes in soil. Environ Geol 2004;45:576-85.

20. Stensmyr MC, Urru I, Collu I, Celander M, Hansson BS, Angioy AM. Rotting smell of dead-horse arum florets - these blooms chemically fool flies into pollinating them. Nature 2002;420:625-6.

21. Aak A, Knudsen GK, Soleng A. Wind tunnel behavioural response and field trapping of the blowfly Calliphora vicina. Med Vet Entomol 2010;24:250-7.

22. Amendt J, Krettek R, Zehner R. Forensic entomology. Naturwissenschaften 2004;91:51-65.

23. Smith KGV. A manual of forensic entomology. London: Department of Entomology, British Museum (Natural History), 1986.

24. Tegoni M, Campanacci V, Cambillau C. Structural aspects of sexual attraction and chemical communication in insects. Trends Biochem Sci 2004;29:257-64.

25. Kaib M. Receptors for meat-odor and flower-odor on antenna of blowfly Calliphora vicina. J Comp Physiol 1974;95:105-21.

26. Huotari M, Mela M. Blowfly olfactory biosensor's sensitivity and specificity. Sensor Actuat B-Chem 1996;34:240-4.

27. Michaud JP, Moreau G. A statistical approach based on accumulated degree-days to predict decomposition-related processes in forensic studies. J Forensic Sci 2011;56:229-32.

28. Castello G, Moretti P, Vezzani S. Retention models for programmed gas chromatography. J Chromatogr A 2009;1216:1607-23.

29. Carter DO, Yellowlees D, Tibbett M. Cadaver decomposition in terrestrial ecosystems. Naturwissenschaften 2007;94:12-24.

30. Matuszewski S, Bajerlein D, Konwerski S, Szpila K. Insect succession and carrion decomposition in selected forests of central Europe. Part 1: pattern and rate of decomposition. Forensic Sci Int 2010;194:85-93.

31. Matuszewski S, Bajerlein D, Konwerski S, Szpila K. Insect succession and carrion decomposition in selected forests of central Europe. Part 2: composition and residency patterns of carrion fauna. Forensic Sci Int 2010;195:42-51.

32. Stoffolano JG. Influence of diapause and diet on development of gonads and accessory reproductive glands of black blowfly, Phormia regina (Meigen). Can J Zool 1974;52:981-8.

33. Weissbecker B, Holighaus G, Schütz S. Gas chromatography with mass spectrometric and electroantennographic detection: analysis of wood odorants by direct coupling of insect olfaction and mass spectrometry. J Chromatogr A 2004;1056:209-16.

34. Vass AA, Barshick SA, Sega G, Caton J, Skeen JT, Love JC, et al. Decomposition chemistry of human remains: a new methodology for determining the postmortem interval. J Forensic Sci 2002;47:542-53.

35. Thomson G. The antoine equasion for vapor-pressure data. Chem Rev 1946;38:1-39.

36. Ritz C, Streibig JC. Bioassay analysis using R. J Stat Softw 2005;12: $1-22$.

37. Kadota HG, Ishida Y. Production of volatile sulfur compounds by microorganisms. Annu Rev Microbiol 1972;26:127-38.

38. Freney JR. Sulfur-containing organics. In: Mac LA, Peterson GH, editors. Soil biochemistry. New York, NY: Marcel Dekker, 1967;229-59.
39. Dainty RH, Edwards RA, Hibbard CM, Marnewick JJ. Volatile compounds associated with microbial-growth on normal and high ph beef stored at chill temperatures. J Appl Bacteriol 1989;66:281-9.

40. Lindiger W, Hansel A, Jordan A. Proton-transfer-reaction mass spectrometry PTR-MS: on-line monitoring of volatile organic compounds at PPTV levels. Chem Soc Rev 1998;27:347-54.

41. Lestremau F, Andersson FAT, Desauziers V. Investigation of artefact formation during analysis of volatile sulphur compounds using solid phase microextraction (SPME). Chromatographia 2004;59:607-13.

42. Haze S, Gozu Y, Nakamura S, Kohno Y, Sawano K, Ohta H, et al. 2-Nonenal newly found in human body odor tends to increase with aging. J Invest Dermatol 2001;116:520-4.

43. Evershed RP, Turnerwalker G, Hedges REM, Tuross N, Leyden A. Preliminary-results for the analysis of lipids in ancient bone. J Archaeol Sci 1995;22:277-90.

44. Vanklinken GJ, Hedges REM. Experiments on C-14 dating of contaminated bone using peptides resulting from enzymatic cleavage of collagen. Radiocarbon 1992;34:292-5.

45. Vanklinken GJ, Bowles AD, Hedges REM. Radiocarbon dating of peptides isolated from contaminated fossil bone-collagen by collagenase digestion and reversed-phase chromatography. Geochim Cosmochim Acta 1994;58:2543-51.

46. Schultz M. Microscopic structure of bone. In: Haglund WD, Sorg MH, editors. Forensic taphonomy, the postmortem fate of human remains. Boca Raton, FL: CRC Press, 2006;187-200.

47. Boumba VA, Ziavrou KS, Vougiouklakis T. Biochemical pathways generating post-mortem volatile compounds co-detected during forensic ethanol analyses. Forensic Sci Int 2008;174:133-51.

48. Combet E, Henderson J, Eastwood CD, Burton KS. Eight-carbon volatiles in mushrooms and fungi: properties, analysis, and biosynthesis. Mycoscience 2006;47:317-26.

49. Kionka C, Kunau WH. Inducible beta-oxidation pathway in Neurospora crassa. J Bacteriol 1985;161:153-7.

50. Heath RJ, Jackowski S, Rock CO. Fatty acid and phospholipid metabolism in prokaryotes. In: Vance DE, Vance JE, editors. Biochemistry of lipids, lipoproteins and membranes. Amsterdam: Elsevier, 2002;55-92.

51. Gottschalk G. Bacterial metabolism. New York, NY: Springer-Verlag, 1986

52. Dourel L, Pasquerault T, Gaudry E, Vincent B. Using estimated on-site ambient temperature has uncertain benefit when estimating postmortem interval. Psyche 2010;2010:1-7.

53. Moller JKS, Hinrichsen LL, Andersen HJ. Formation of amino acid (L-leucine, L-phenylalanine) derived volatile flavour compounds by Moraxella phenylpyruvica and Staphylococcus xylosus in cured meat model systems. Int J Food Microbiol 1998;42:101-17.

54. Derrick S, Large PJ. Activities of the enzymes of the ehrlich pathway and formation of branched-chain alcohols in Saccharomyces cerevisiae and Candida utilis grown in continuous-culture on valine or ammonium as sole nitrogen-source. J Gen Microbiol 1993;139:2783-92.

55. Chen ECH. Relative contribution of ehrlich and biosynthetic pathways to the formation of fusel alcohols. J Am Soc Brew Chem 1978;36:39 $-43$.

56. Stahnke LH. Volatiles produced by Staphylococcus xylosus and Staphylococcus carnosus during growth in sausage minces-part II. The influence of growth parameters. Food Sci Technol-LEB 1999;32:365-71.

57. Zhang CH, Hirano T, Suzuki T, Shirai T. Studies on the odor of fishes. 2. Enzymatically generated specific volatile compounds in ayu tissues. Nippon Suisan Gakk 1992;58:559-65.

58. Thakeow P, Angeli S, Weissbecker B, Schütz S. Antennal and behavioral responses of Cis boleti to fungal odor of Trametes gibbosa. Chem Senses 2008;33:379-87.

59. Borjesson TS, Stollman UM, Schnurer JL. Off-odorous compounds produced by molds on oatmeal agar-identification and relation to other growth-characteristics. J Agri Food Chem 1993;41:2104-11.

60. Pasanen AL, Lappalainen S, Pasanen P. Volatile organic metabolites associated with some toxic fungi and their mycotoxins. Analyst 1996;121:1949-53.

61. Korpi A, Pasanen AL, Pasanen P. Volatile compounds originating from mixed microbial cultures on building materials under various humidity conditions. Appl Environ Microbiol 1998;64:2914-9.

62. Sunesson AL, Nilsson CA, Andersson B, Blomquist G. Volatile metabolites produced by two fungal species cultivated on building materials. Ann Occup Hyg 1996;40:397-410.

63. Larsen TO, Frisvad JC. Comparison of different methods for collection of volatile chemical markers from fungi. J Microbiol Methods $1995 ; 24: 135-44$ 
64. Hadar Y, Dosoretz CG. Mushroom mycelium as a potential source of food flavour. Trends Food Sci Technol 1991;2:214-8.

65. Feussner I, Kühn H, Wasternack C. Do specific linoleate 13-lipoxygenases initiate ß-oxidation? FEBS Lett 1997;406:1-5.

66. Smith EA, Macfarlane GT. Enumeration of human colonic bacteria producing phenolic and indolic compounds: effects of $\mathrm{pH}$, carbohydrate availability and retention time on dissimilatory aromatic amino acid metabolism. J Appl Bacteriol 1996;81:288-302.

67. Cummings JH, Hill MJ, Bone ES, Branch WJ, Jenkins DJA. Effect of meat protein and dietary fiber on colonic function and metabolism. 2. Bacterial metabolites in feces and urine. Am J Clin Nutr 1979;32:2094-101.

68. Bone E, Tamm A, Hill M. Production of urinary phenols by gut bacteria and their possible role in causation of large bowel cancer. Am J Clin Nutr 1976;29:1448-54.
69. Paczkowski S, Schütz S. Post-mortem volatiles of vertebrate tissue. Appl Microbiol Biotechnol 2011;91:917-35.

Additional information and reprint requests:

Sebastian Paczkowski, M.Sc.

Department of Forest Zoology and Forest Conservation

Büsgeninstitut, Georg-August-University

Göttingen

Germany

E-mail: spaczko@gwdg.de 


\section{Volatile emission of decomposing pig carcasses (Sus scrofa domesticus L.) as an indicator for the post mortem interval}

Manuscript short title: VOLATILE EMISSION AS A PMI INDICATOR

Authors:

Sebastian Paczkowski ${ }^{1}$ (Corresponding author); (S. Paczkowski), M.Sc., Dept. of Forest Zoology and Forest Conservation, Büsgen-Institute, Georg-August-University, Göttingen, Germany, Büsgenweg 3, 37077 Göttingen, Germany; e-Mail: spaczko@gwdg.de; phone: +49-551-39-3610; fax: +49-551-3922089

Friederike Maibaum (F. Maibaum), M.Sc., Dept. of Forest Zoology and Forest Conservation, BüsgenInstitute, Georg-August-University, Göttingen, Germany, Büsgenweg 3, 37077 Göttingen, Germany; e-Mail: fmaibau@gwdg.de

Sara Nicke, (S. Nicke), Technical Assistant, Dept. of Forest Zoology and Forest Conservation, BüsgenInstitute, Georg-August-University, Göttingen, Germany, Büsgenweg 3, 37077 Göttingen, Germany; e-Mail: snicke@gwdg.de

Henrik Ziegenhagen, (H. Ziegenhagen), B.Sc., Dept. of Forest Zoology and Forest Conservation, Büsgen-Institute, Georg-August-University, Göttingen, Germany, Büsgenweg 3, 37077 Göttingen, Germany; e-Mail: henrik.ziegenhagen@stud.uni-goettingen.de

Stefan Schütz (S. Schütz), Prof. Dr., Dept. of Forest Zoology and Forest Conservation, BüsgenInstitute, Georg-August-University, Göttingen, Germany, Büsgenweg 3, 37077 Göttingen, Germany; e-Mail: stefan.schuetz@forst.uni-goettingen.de

\footnotetext{
${ }^{1}$ The work of Sebastian Paczkowski was kindly supported by the Cusanuswerk, Bischöfliche Studienstiftung, Germany.
} 


\begin{abstract}
In this study, core volatiles of vertebrate decay were selected and evaluated for a volatile based estimation of the post mortem interval. Such volatiles should $\left(1^{\text {st }}\right)$ be reliably emitted during vertebrate decay, $\left(2^{\text {nd }}\right)$ be emitted in high quantities, and $\left(3^{\text {rd }}\right)$ show a reproducible quantitative dynamic during the decaying process. Four pigs (Sus scrofa domesticus L.) were placed in a deciduous forest in Germany at different times in the year. Volatiles released during their decomposition were sampled. Seventeen compounds were identified and quantified by GC-MS. Electrophysiological experiments on the antenna of female Calliphora vicina were used as an evolutionary tuned information filter to evaluate the $1^{\text {st }}$ criterion. Dimethyl disulfide and dimethyl trisulfide fulfilled all three criteria. The role of butan-1-ol, phenol, and aldehydes for PMI estimation is discussed, as well as correlations between volatile emissions, $\mathrm{ADD}_{5}$ and observed stages of decay.
\end{abstract}

Keywords: Forensic entomology, Forensic chemoecology, decompositional process, stages of decay, core volatiles, volatile emission pattern, Calliphora vicina, electrophysiology, electroantennogram, EAG, olfaction, VOC, electronic noses, semi-conductor gas sensors

\title{
Introduction
}

Vertebrates consist of many different tissues like bones, muscles, skin or the nervous system. After death, these tissues are degraded by reactive oxygen species, autolysis, microorganisms or scavengers (Paczkowski and Schütz, 2011). The chemical degradation during autolysis or microbial activity yields volatiles as by- or end products (Vass et al., 2008; Vass et al., 2004; Vass et al., 2002; Statheropoulos et al., 2007; Statheropoulos et al., 2005; Hoffman et al., 2009; Dekeirsschieter et al., 2009; Paczkowski and Schütz, 2011; Paczkowski et al., 2012; DeGreef and Furton, 2011). These volatile products are in the focus of recent research, because their emission might be correlated to the post mortem interval (PMI). Most of the established methods for PMI estimation are useful in the first week after death. However, the process of decay is influenced by temperature, moisture, or other factors like clothing or drug consumption. Therefore, PMI estimation results in time spans rather than time points.

The most important method for PMI estimation is the outward appearance of the cadaver. This appearance is classified in stages. The fresh stage of decomposition is characterized by a lowering body temperature, rigor mortis, and the formation of blue or black spots on the skin. During this stage autolysis, reactive oxygen species and microbes start to decompose the cadaver. Volatile products of this decomposition cause the bloating of the intestine at the onset of the bloated stage. Increasing microbial activity, the feeding of insect larvae, or scavengers cause the breaking of the skin and the outflow of the inner fluid. This is the beginning of the active stage. The weight loss in this stage is the highest during the decaying process (Carter et al., 2007) and the rate of the weight loss is probably influenced by the soil humidity (Matuszewski et al., 2010a). When most of the tissue is digested by larvae, metabolized by microorganisms, or drained off, the cadaver is in the advanced stage of decay. In this stage the cadaver is drier and insect activity is lower than in the active or bloated stage. In the dry stage of decay only bones and hair are left. The limitations of the stages approach is discussed by several authors (Carter et al., 2007; Matuszewski et al., 2010b), as the optical impression is subjective and a cadaver can show characteristics of different stages of decay at 
the same time. The head can show a higher rate of decomposition than other parts of the cadaver. While the bowel is still bloated, the cadaver could deflate at the anus.

Forensic entomology offers additional cues for PMI estimation (Amendt et al., 2004). Insect colonization of dead vertebrates shows a time dependent succession pattern (Matuszewski et al., 2010a). The development of insect larvae could allow PMI estimation in the time span of one month after death (Amendt et al., 2004). The larval growth is influenced by the temperature and, therefore, additional data of weather stations is required in order to estimate the rate of the larval development. It has been shown that data of weather stations are not always sufficient for PMI estimation (Dourel et al., 2010). A combination of methods can contribute to improved PMI estimation. Volatile based PMI estimation is proposed to complement established methods (Dekeirsschieter et al., 2009; Paczkowski and Schütz, 2011; Paczkowski et al., 2012; LeBlanc and Logan, 2010; Statheropoulos et al., 2007; Statheropoulos et al., 2005; Vass et al., 2008; Vass et al., 2004).

The complex chemical processes during the decay of a vertebrate can yield many different volatiles. A volatile based PMI estimation should rely on volatiles, which are reliably present during the decay of vertebrates. A first approach to find such core volatiles was done by Dekeirsschieter et al., 2009. They analyzed the volatiles of decaying pig cadavers (Sus scrofa domesticus) in different habitats (rural, forest, and urban) and identified tentative core volatiles of decay. As indicated by the authors, many experiments are required in order to confirm these core volatiles, because different vertebrate species may yield different volatile pattern and environmental aspects can influence the volatile emission. The olfaction of forensically important insects can be utilized for the identification of core volatiles in order to avoid time consuming and costly experiments. These insects are adapted to perceive volatiles of decay (Paczkowski et al., 2012; Kalinova et al., 2009; Hoermann et al., 2011; LeBlanc and Logan, 2010). The females find dead vertebrates for oviposition by means of olfaction and depend on reliably emitted volatiles (Huotari, 2000). Such volatiles can be useful for volatile based PMI estimation.

The cadaveric volatiles perceived by the blowfly Calliphora vicina should fulfill three criteria: The first criterion is the emission of the volatile by any kind of decaying vertebrate and, in consequence, by any human corpse. This criterion is important for the reliability of the method, as vertebrate species and environment can alter the volatile emission (Dekeirsschieter et al., 2009). Such environmental influences could be microclimatic conditions during the decaying process that favor different microbial species with different volatile by- or end products. Moreover, feeding of scavengers might influence the rate of decomposition or could introduce different microbial species from the scavengers mouth region (Paczkowski and Schütz, 2011). Alterations in the volatile profile caused by clothing or drug consumption are not considered in this study, because such environmental influences were not part of the ecologically driven adaption of $C$. vicina. The second criterion requires a high concentration of the volatile to enable the detection with GC sampling techniques or artificial noses. The metabolic activity of microbes and the vapor pressure of volatiles depend on the temperature in a non linear way (Goll et al., 1983; Thomson, 1946). Therefore, a volatile which is emitted in low concentration at $15^{\circ} \mathrm{C}$ might drop below the detection threshold at $5^{\circ} \mathrm{C}$. Moreover, core volatiles meeting the first and the second criterion might be important for search operations with trained canines or prospective artificial noses. The third criterion for volatiles enabling PMI 
estimation is the reproducible dynamic of their emission. The increase or decrease of core volatiles should show the same correlation to the PMI for any decaying process of vertebrates. Calculating the ADD can improve this correlation, because the ADD takes in account the temperature dependence of the decaying process.

In this study, the volatile emission patterns of decaying pig carcasses (Sus scrofa domesticus L.) are investigated in order to identify volatiles, which fulfill all of the three criteria. The olfaction of female blowflies (C. vicina) is used as an evolutionary tuned filter for the improved selection of these volatiles.

\section{Materials and Methods}

\section{Collection of volatiles from decaying pigs}

Four domestic pigs (Sus scrofa domesticus L.) $(25-30 \mathrm{~kg}$ ) were placed in a deciduous forest near Klein Schneen, Lower Saxony, Germany, in the time period from 2008 to 2011. The pigs were ordered from a pig farm and killed by penetrative captive bolt. Approval from the university's Animal Ethics Committee was obtained prior to killing the pigs. Domestic pigs were used, because they resemble a human body according to the relative size of the inner organs and the chemical composition of the tissues $(8,11)$. The pig cadavers were placed in a metal fence casing, which was fastened to the ground to protect the cadavers against scavengers. To collect the volatiles, TDS tubes filled with TENAX ${ }^{\circledR}$ (TDS; Gerstel, Mühlheim, Germany) were connected to membrane pumps (Thomas Division, Sheboygan, USA) and placed $2 \mathrm{~cm}$ above the cadavers. The position of the tubes was selected on the basis of the optical impression of the decaying stage. The characteristics of these stages (fresh, bloated, active, and advanced decay) were critically reviewed by Carter et al., 2007 (Carter et al., 2007) and applied by Dekeirsschieter et al., 2009 (Dekeirsschieter et al., 2009) and Statheropoulos et al., 2011 (11). In the fresh and bloated stage of decay the tubes were places above the head or the swollen parts of the cadaver. In the active or advanced stage of decay the tubes were placed above the stomach or the anal region. The $3^{\text {rd }}$ and $4^{\text {th }}$ pig showed the mosaic-effect described by Matuszewski et al., 2010 (Matuszewski et al., 2010b). While the head of these pigs was in a progressed stage of decay, the torso was still bloated. In case of the $3^{\text {rd }}$ pig, the tube was positioned above the head and in case of the $4^{\text {th }}$ pig the tube was positioned above the swollen torso in order to evaluate the influence of the sampling tubes' position on the sampled volatile profile. The pumps withdrew air through the TDS tubes for two hours with a rate of $70 \mathrm{~L} / \mathrm{h}$. During the whole period of sample collection, the temperature was recorded with a $\mathrm{HOBO}$ C data logger (Onset Computer Corporation, Bourne, USA). The accumulative degree days $\left(\mathrm{ADD}_{5}\right)$ were calculated by dividing the sum of the minimum and maximum temperature $\left({ }^{\circ} \mathrm{C}\right.$ ) per day by two and subtracting 5 (Michaud and Moreau, 2011). Controls were placed $50 \mathrm{~m}$ away from the decaying pigs to collect the volatiles of the sampling site.

\section{Trace analysis with the TDS samples from decaying pigs}

The TDS GC-MS trace analytic was performed according to a method described in Paczkowski et al., 2012 (p.71). The identified compounds were quantified from the peak areas of selected single ions in the sample. Dimethyl trisulfide was quantified on the basis of $\mathrm{m} / \mathrm{z}=126$; dimethyl disulfide $\mathrm{m} / \mathrm{z}=94$; nonanal, $\mathrm{m} / \mathrm{z}=57$; hexan-1-ol, $\mathrm{m} / \mathrm{z}=56 ; 1$-octen-3-ol, $\mathrm{m} / \mathrm{z}=57 ; 3$-methylbutan-1-ol, $\mathrm{m} / \mathrm{z}=55$; 
heptanal, $\mathrm{m} / \mathrm{z}=70$; octan-3-one, $\mathrm{m} / \mathrm{z}=43$; acetophenone, $\mathrm{m} / \mathrm{z}=105$; indole, butanoic acid $\mathrm{m} / \mathrm{z}=60$; $\mathrm{m} / \mathrm{z}=117 ;$ phenol, $\mathrm{m} / \mathrm{z}=94 ; 4$-methylphenol, $\mathrm{m} / \mathrm{z}=107 ; 3$-methyl-butanoic acid $\mathrm{m} / \mathrm{z}=60$; acetic acid, $\mathrm{m} / \mathrm{z}=43$; butan-1-ol, $\mathrm{m} / \mathrm{z}=56$; and undecane, $\mathrm{m} / \mathrm{z}=57$. The samples were grouped by the optical impression of the stages of decay: fresh, bloated, active decay, and advanced decay. The correlation between the optical impression, the $A D D_{5}$ and the relative abundance of selected compounds in the volatile profile was evaluated.

\section{Collection and rearing of flies}

In the summer of 2011, female $C$. vicina were caught from fresh ground meat (half pork, half beef) in the city of Göttingen. The species $C$. vicina was chosen as it is one of the first to arrive on a dead vertebrate, and is present during all stages of decay. This species is important for forensic entomology (Amendt et al., 2004; Smith, 1986) and is a model organism for research on the olfaction of blowflies (Kaib, 1974; Huotari and Mela, 1996; Stensmyr et al., 2002). After removing the antennae for electrophysiological experiments, the development of the ovaries was assessed (Stoffolano, 1974). This was used as an indicator of the predilection of the flies to oviposit and, therefore, exhibit high electrophysiological performance. Only flies with developed ovaries were selected for electrophysiological measurements.

\section{Electroantennogram (EAG) with C. vicina}

The EAG experiments were performed according to the method used in Paczkowski et al., 2012 (p.72).

\section{Results}

\section{Decaying process}

The temperature regime during the decay of the four pigs and the calculated $A D D_{5}$ for the onsets of the decaying stages are shown in Figure 1. The average day temperature varied between $3{ }^{\circ} \mathrm{C}$ and $18^{\circ} \mathrm{C}$ in autumn for the first pig, $7^{\circ} \mathrm{C}$ and $30^{\circ} \mathrm{C}$ in early summer for the second pig, $10^{\circ} \mathrm{C}$ and $29^{\circ} \mathrm{C}$ in late summer for the third pig, and $11{ }^{\circ} \mathrm{C}$ and $25^{\circ} \mathrm{C}$ in spring for the fourth pig. The onset of the bloated stage varied between 7 and 12 days, the onset of the active stage between 14 and 24 days, and the onset of the advanced stage between 21 and 43 days. The calculated $\mathrm{ADD}_{5}$ varied between 38 and 121 for the onset of the bloated stages, between 136 and 180 for the active stages, and between 242 and 343 for the advanced stages.

Frequent rain kept the cadaver and the habitat moist during the decay of the first pig. Although in the fresh stage few flies (Calliphoridae, Muscidae) were observed on the cadaver, eggs were found on snout and anus of the pig. Larvae hatched few days before and in the bloated stage. In the active stage of decay the cadaver was still swollen in the region of the intestine and many larvae were present on the fluid besides head, back, and anus. In the advanced stage of decay very few flies visited the still moist cadaver and fewer larvas were present on the remains.

Frequent rainfalls kept the second pig moist, as well. Fly activity was low and few eggs were laid on the snout and the back of the pig. The moist weather led to a high activity of snails, which fed on the eggs. Therefore, no larvae hatched on the snout and the back. Larvae of Silphidae hatched beneath 
the cadaver. In the bloated stage the cadaver swelled and the skin broke up at some places, which were damaged by the feeding of the snails.

These places were attractive for Calliphoridae, Muscidae and Silphidae, but no eggs were found there. All of these insect families were abundant in the mouth opening in the bloated stage, as well and eggs were laid on the back and the snout of the cadaver. The swollen stomach got black and broke up at the onset of the active decay and beetle larvae (Silphidae) came out from underneath the cadaver. In the advanced stage of decay the whole cadaver was still moist. There was almost no activity of flies and beetles and only few Silphidae larvae were left on the tissue.

There were no rainfalls during the fast decay of the third pig (Figure 1). Few flies (Calliphoridae) visited the cadaver in the short period of the fresh stage (Figure 1), while many flies (Calliphoridae, Muscidae) and beetles (Staphinilidae, Silphidae) visited the cadaver in the bloated stage of decay. The head showed the characteristics of advanced decay, while most of the cadaver was still in the bloated stage. Many larvae were found in the mouth opening and on the breast. In the active decaying stage, the head was in the dry stage of decay and the larvae migrated to the fluid on top of the stomach region. Many flies (Calliphoridae, Muscidae) visited the cadaver in this stage. Only few larvae were visible on the cadaver in the advanced stage. 


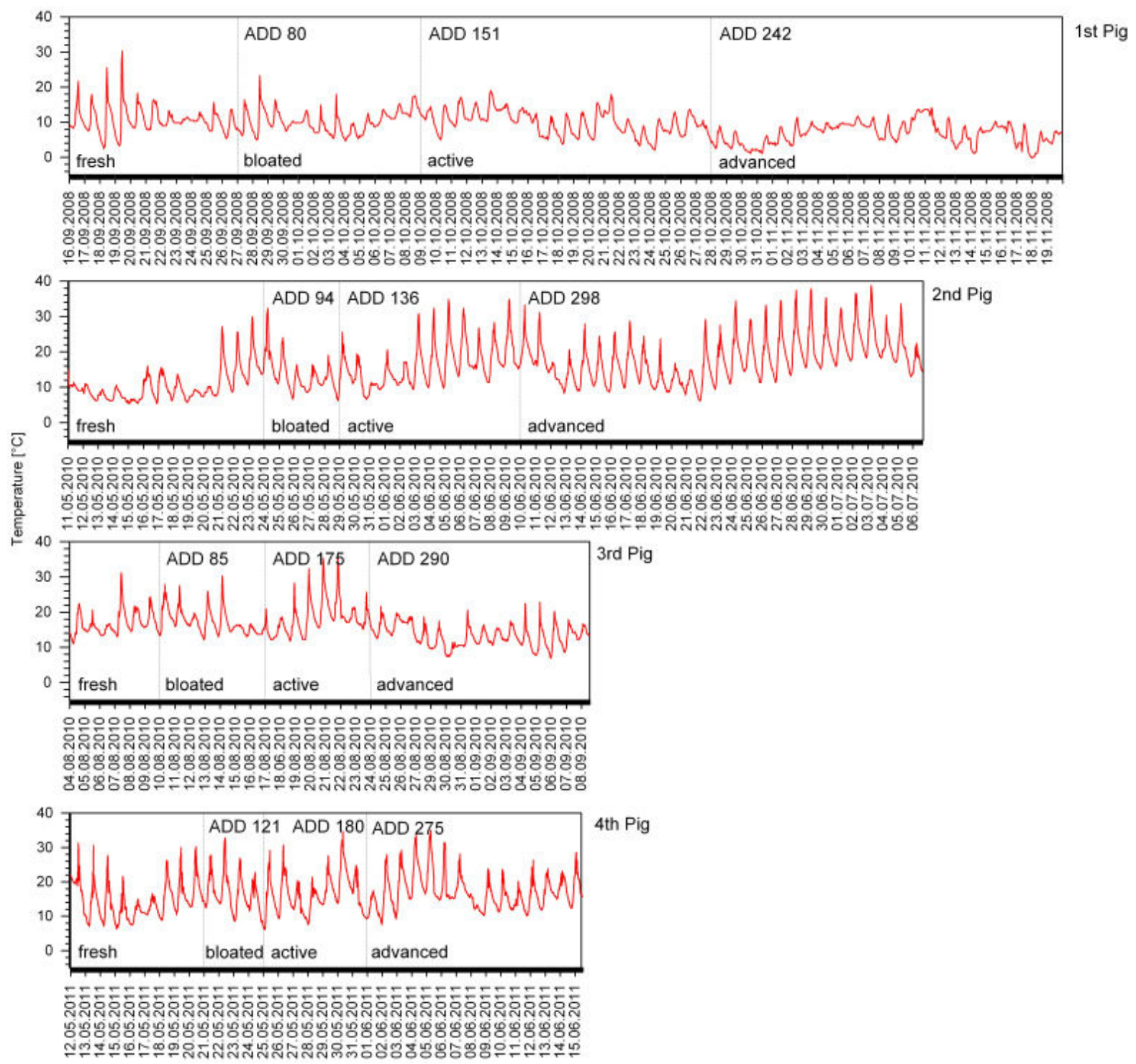

Figure 1 shows the temperature regime, the stages of decay and the assigned ADD value (accumulated degree days) for the four pigs.

The weather was dry during the fresh, bloated, and active stages of the fourth pig's decay. Calliphoridae visited the cadaver in the fresh stage of decay. Eggs were laid on the head, the anus, and in the mouth opening. More insects (Calliphoridae, Muscidae, Silphidae) were attracted in the bloated stage and many larvae were present on the head, the back, and the anus. Eggs were found on the legs. The anus region broke up at the onset of the active decay, the region of the intestine was still swollen and the head showed characteristics of advanced decay. The tissue turned moist and grey after a rainfall in the advanced stage of decay. Very few larvae or adult insects visited the cadaver in this stage.

\section{Trace analysis of the TDS samples from decaying pigs}

Seventeen compounds were identified and quantified during the decay of the four pigs (Table 1). Among these compounds there are four alcohols (24\%), three acids (18\%), two sulfur compounds (12\%), two aldehydes (12\%), two aromatics (12\%), one ketone (6\%), and one aromatic heterocycle (6\%). Table 2 shows the quantity of these compounds assigned to the stages of decay. No compound 
was reproducibly detected during the fresh stage of decay. However, the group of aldehydes (heptanal and nonanal) is present at low absolute concentrations (0-85 ng) in most samples of the fresh stage and only the first sample of the $2^{\text {nd }}$ pig is an exception. Acetophenone and dimethyl disulfide were reproducibly detectable in the bloated stage of decay. Acetophenone is emitted at low concentration (5-43 ng) and dimethyl disulfide is emitted at increasing concentration (2-442 ng). Seven compounds (dimethyl disulfide, dimethyl trisulfide, heptanal, phenol, undecane, butan-1-ol, and acetophenone) were reproducibly detected in the active stage of decay. The sulfur compounds, phenol, and butan-1-ol show strongly increasing concentrations (sulfur compounds: 581748 ng; phenol: 6-2,448 ng, 193-5,198 ng) in this stage. Phenol, butan-1-ol, and acetophenone were reproducibly detected in the advanced stage of decay. Butan-1-ol is emitted at varying concentrations in most of the samples of this stage $(9-3,035 \mathrm{ng})$ and phenol is emitted at high concentrations in all of the samples of this stage (316-3,256 ng).

\section{Electroantennogram (EAG) with C. vicina}

The results of the electrophysiological experiments are listed in Table 3. Additional data is published in Paczkowski et al., 2012 (p.76). Fourteen of the seventeen compounds were electrophysiological active on the antennae of female $C$. vicina. Both sulfur compounds showed the highest antennal responses and were significantly distinguishable from control stimuli down to dilutions of $10^{-8} \mathrm{~g} / \mathrm{g}$ (dimethyl disulfide) and $10^{-9} \mathrm{~g} / \mathrm{g}$ (dimethyl trisulfide). Both aldehydes showed high antennal responses, as well as the three alcohols hexan-1-ol, 1-octen-3-ol, and 3-methylbutan-1-ol. These compounds were significantly distinguishable from control stimuli down to dilutions of $10^{-4} \mathrm{~g} / \mathrm{g}$ and $10^{-5} \mathrm{~g} / \mathrm{g}$. Octan-3-one, acetophenone, indole, butanoic acid, phenol, 4-methylphenol, and 3methylbutanoic acid were significantly distinguishable from control stimuli down to dilutions of $10^{-3}$ $\mathrm{g} / \mathrm{g}$ and $10^{-2} \mathrm{~g} / \mathrm{g}$. However, acetic acid, butan-1-ol, and undecane elicited no significant electrophysiological responses.

\section{Discussion}

The aim of this study was the identification of volatiles of the decaying process, which fulfill all of the three criteria (1: reliably present; 2 : high quantities; 3 : reproducible dynamic). The volatiles that fulfil these criteria can be detected by technical sensors, which are much less sensitive than insect antennae. Seven compounds fulfill the first criterion in this study. 
Table 1 lists the compounds of pig decay (S. scrofa domesticus) identified by mass spectra and confirmed by standard compounds in the order of their linear retention index on a nonpolar column. References are listed that identified the same compounds, respectively.

No. Compound

TDS (LRI) Chemical

1 Acetic acid

2 Butan-1-o

Dimethyl disulfide

3-Methylbutan-1-ol

Butanoic acid

$6 \quad 3-M e t h y l-b u t a n o i c$ acid

7 Hexan-1-o

$8 \quad$ Heptanal

9 Dimethyl trisulfide

10 1-Octen-3-o

11 Octan-3-one

12 Phenol

13 Acetophenone

14 Undecane

15 4-Methylpheno

16 Nonana

17 Indole classes

759

Authentic standards

(company, purity,

\section{CAS No.)}

Fluka, 98\%, 64-19-7

Fluka, 98\%, 71-36-3

Alcohol

Sulfur compound

Alcohol

Acid

Acid

884 Alcohol

906 Aldehyde

976

Sulfur

compound

Alcohol

Ketone

Aromatic

Aromatic

Alkane

Aromatic

Aldehyde

1331 Aromatic heterocycle

SAFC, 98\%, 503-74-2

VWR, 96\%, 106-68-3

Aldrich, 99\% 98-86-2

Fluka, 99\%, 120-72-9

\section{Literature}

Gottschalk, 1986; Dent et al., 2004; Boumba et al., 2008; Hoermann et al., 2011; DeGreef and Furton, 2011)

(Dekeirsschieter et al., 2009; Statheropoulos et al., 2005; O'Neal and Poklis, 1996; Gill-King, 1999; Dent et al., 2004; Boumba et al., 2008)

Merck, 98\%, 624-92-0

(Stensmyr et al., 2002; Dent et al., 2004; Vass et al., 2008; Vass et al., 2004; Statheropoulos et al.

2007; Statheropoulos et al., 2005; Hoffman et al., 2009; Dekeirsschieter et al., 2009; Stutz et al., 1991; Stutz et al., 1991; Lorenzo et al., 2003; Podskalska et al., 2009)

Aldrich, 99,5\%, 123-51-3 (Dekeirsschieter et al., 2009; O'Neal and Poklis, 1996; Boumba et al., 2008; Derrick and Large, 1993;

Aldrich, 98\%, 107-92-6

Aldrich, 98\%, 111-27-3

Acros, 95\%, 111-71-7

SAFC, 98\%, 3658-80-8

Merck, 98\%, 3391-86-4

Aldrich, 99\%, 108-95-2

Acros, 99\%, 1120-21-4

Aldrich, 99\%, 106-44-5

Merck, 98\%, 124-19-6
Ercolini et al., 2009)

(Dekeirsschieter et al., 2009; Statheropoulos et al., 2005; Hoffman et al., 2009; Gill-King, 1999; Lorenzo et al., 2003; Boumba et al., 2008; Dent et al., 2004; Hoermann et al., 2011)

(Hoermann et al., 2011)

(Kaib, 1974; Huotari, 2000; Statheropoulos et al., 2005; Hoffman et al., 2009; Stutz et al., 1991; Haze et al., 2001)

(Hoffman et al., 2009; Dekeirsschieter et al., 2009; Stutz et al., 1991; Vass et al., 2008; Haze et al., 2001; Lorenzo et al., 2003)

(Stensmyr et al., 2002; Dent et al., 2004; Vass et al., 2008; Vass et al., 2004; Statheropoulos et al., 2007; Statheropoulos et al., 2005; Dekeirsschieter et al., 2009; Stutz et al., 1991; Podskalska et al., 2009; DeGreef and Furton, 2011)

(Hoffman et al., 2009; Ercolini et al., 2009; Zhang et al., 1992; Combet et al., 2006)

(Statheropoulos et al., 2007; Dekeirsschieter et al., 2009; Vass et al., 2008; Hoffman et al., 2009; Cummings et al., 1979; DeGreef and Furton, 2011

(Dekeirsschieter et al., 2009; Statheropoulos et al., 2007)

(Vass et al., 2004; Vass et al., 2008; DeGreef and Furton, 2011)

(Dekeirsschieter et al., 2009; Statheropoulos et al., 2007; Geypens et al., 1997)

(Vass et al., 2008; Vass et al., 2004; Hoffman et al., 2009; Haze et al., 2001; Ercolini et al., 2009;

(Dent et al., 2004; Hoffman et al., 2009; Dekeirsschieter et al., 2009; Vass et al., 2002; O'Neal and Poklis, 1996; Statheropoulos et al., 2005; Lorenzo et al., 2003; Gill-King, 1999; Evans, 1963; O'Neal and Poklis, 1996) DeGreef and Furton, 2011) 
Table 2: Emission quantity of volatiles. The table shows the quantity of the volatile compounds in the TDS samples in ng. The order of the numbers is: median, lower quartile, upper quartile, minimum, maximum. The median displays the average concentration in all samples of each stage. The $\mathbf{N}$ value shows the number of samples analyzed in each stage of decay. A minimum of" 0 " shows that the compound was absent in at least one sample.

$\begin{array}{lcccc}\text { Compound } & \text { Fresh (N=14) } & \text { Bloated } \mathbf{( N = 9 )} & \text { Active (N=12) } & \text { Advanced (N=19) } \\ \text { Dimethyl trisulfide } & - & 1,0,5,0,849 & 256,39,545,10,1495 & 83,14,193,0,1686 \\ \text { Dimethyl disulfide } & - & 5,3,51,2,442 & 133,61,389,47,441 & 86,20,441,0,2053 \\ \text { Nonanal } & 5,2,13,0,47 & 4,0,20,0,56 & 10,1,29,0,33 & 6,2,15,0,92 \\ \text { Hexan-1-ol } & 0,0,0,0,7 & 0,0,2,0,15 & 0,0,0,0,22 & 0,0,0,0,16 \\ \text { 1-Octen-3-ol } & - & - & - & - \\ \text { 3-Methylbutan-1-ol } & 0,0,0,0,2 & 3,0,12,0,23 & 99,4,125,0,423 & 19,3,57,0,330 \\ \text { Heptanal } & 6,5,10,0,38 & 6,1,8,0,94 & 11,7,37,3,112 & 6,1,44,0,263 \\ \text { Octan-3-one } & 0,0,2,0,33 & 0,0,2,0,5 & 0,0,0,0,4 & 0,0,0,0,6 \\ \text { Indole } & - & - & 1,0,4,0,15 & 5,0,22,0,695 \\ \text { Phenol } & 3,0,6,0,10 & 2,0,11,0,1594 & 146,21,1045,6,2448 & 709,426,2273,316,3256 \\ \text { Undecane } & 2,1,3,0,14 & 2,1,3,0,3 & 6,3,15,1,58 & 4,2,13,0,33 \\ \text { Butan-1-ol } & - & 0,0,0,0,76 & 1403,275,2423,193,5198 & 632,61,1368,9,3035 \\ \text { 3-Methyl-butanoic acid } & - & 0,0,167,0,2140 & 98,0,1069,0,11578 & 365,0,2649,0,26063 \\ \text { Butanoic acid } & - & 0,0,0,0,2 & 0,0,1,0,7 & 0,0,1,0,40 \\ \text { 4-Methylphenol } & - & 0,0,0,0,62 & 4,0,59,0,112 & 6,3,23,0,195 \\ \text { Acetophenone } & 19,12,28,0,56 & 28,9,40,5,43 & 24,13,46,8,54 & 18,8,36,6,60 \\ \text { Acetic acid } & 0,0,15,0,112 & 0,0,33,0,69 & 0,0,119,0,510 & 21,0,97,0,328\end{array}$

The volatile compounds dimethyl disulfide, dimethyl trisulfide, heptanal, phenol, undecane, acetophenone, and butan-1-ol were reproducibly emitted during the bloated, active or advanced stages of decay. This is indicated in Table 2 by a minimum higher than zero. The olfaction of female $C$. vicina is used in order to confirm the reliability of these compounds as core volatiles for vertebrate decay. Electrophysiological activity of a volatile does not necessarily implicate a sufficient concentration for electronic sensor systems ( $2^{\text {nd }}$ criterion), because insect olfaction is much more sensitive in comparison to technical systems (Weissbecker et al., 2004, O'Neal and Poklis 1996). Furthermore, electrophysiological activity does not suggest a correlation with one or several stages of decay or with the calculated $A D D_{5}$ values ( $3^{\text {rd }}$ criterion), because the fly is a generalist and does not necessarily differentiate between the bloated, active, and advanced stage of decay by means of volatiles. The applicability and stage specificity of volatiles has to be evaluated by the $2^{\text {nd }}$ and the $3^{\text {rd }}$ criterion.

Among the seven core volatile candidates, butan-1-ol and undecane are not electrophysiological active. Although butan-1-ol has been detected during the decay of vertebrates several times ( $O$ ' Neal and Poklis, 1996; Gill-King, 1999; Dent et al., 2004; Statheropoulos et al., 2005; Dekeirsschieter et al., 2009; Paczkowski et al., 2012) it is not perceived by the blowfly. C. vicina might not need to detect volatiles, which are important for the differentiation between the bloated and the active stage. The biology of this insect shows that the fly is a generalist among invertebrate carrion visitors. Adults can visit carrions in the fresh stage of decay, but can be found in larger numbers in the bloated and active stage of the decaying process (Smith, 1986; Matuszewski et al., 2010a). Larvae of $C$. vicina develop successfully in both stages of decay. The fly does not have to differentiate between the bloated and active stage of decay and, therefore, does not have to perceive butan-1-ol. In contrast, the male necrophagous hide beetle Dermestes maculatus does differentiate between the bloated and the 
Table 3: EAG. The Table shows the dose-response relationship of female $C$. vicina antennae. The order of the numbers is: Median (upper quartile, lower quartile). The antennal responses are given in $\mathrm{mV}(\mathrm{N}=3)$. The T-test or U-test was used to calculate significant differences between the dilution and the paraffin oil control. The p-level was: $*^{*}<0.05 ;{ }^{*}<0.005$; $* * *<0.001$. Most of the dose-response relationships have already been published in Paczkowski et al., (2012) (p.76).

$\begin{array}{lcccccc}\text { Compound } & \begin{array}{c}\text { Paraffin } \\ \text { oil }\end{array} & 10^{-6} & 10^{-5} & 10^{-4} & 10^{-3} & 10^{-2} \\ & & & & & * * & * * * \\ \text { Acetophenone } & 13(9,23) & 14(11,28) & 15(10,20) & 16(12,22) & 29(28,65) & 57(56,123) \\ & & & & & * & * \\ \text { Butanoic acid } & 13(9,23) & 14(11,23) & 17(12,26) & 23(19,26) & 25(21,46) & 26(25,37) \\ & & & & & & * \\ \text { 4-Methylphenol } & 16(9,23) & 12(10,30) & 12(11,26) & 16(14,21) & 20(18,32) & 25(22,64) \\ \text { 3-Methyl-butanoic } & 17(16,27) & 16(14,27) & 16(14,21) & 18(15,22) & 20(18,33) & 21(19,35) \\ \text { acid } & & & & & & \\ \text { Acetic acid } & 17(14,27) & 12(11,41) & 16(14,25) & 19(17,26) & 19(18,33) & 30(22,34)\end{array}$

active stage of decay by means of volatiles. The male beetle perceives benzyl butyrate, which is regarded as a product of the butanoic acid fermentation of glucose by the obligate anaerobic Clostridiaceae (Hoermann et al., 2011). Butanoic acid fermentation of Clostridiaceae can shift from butanoic acid production to butan-1-ol production (Boumba et al., 2008).

All of this information suggests an important role of butan-1-ol for the volatile based differentiation between the bloated and the active stage of decay and the compound's emission is correlated to the onset of the active stage in this study, as well (Figure 2). Undecane is neither known to be detected by C. vicina (Table 3) or other necrophilic insects (Kalinova et al., 2009; Hoermann et al., 2011), nor frequently identified in other trace analytical studies on vertebrate decay (Paczkowski and Schütz, 2011). Therefore, this compound is excluded as a core volatile.

The second criterion (high concentration) is fulfilled by dimethyl disulfide, dimethyl trisulfide, phenol, and butan-1-ol. These compounds are the most prominent ones in the volatile pattern of the decaying pigs (Table 2). The electrophysiological active aldehydes heptanal and nonanal are emitted at low concentration in comparison to these compounds. However, it has already been shown in a study on decaying mouse carcasses that aldehydes can enable a differentiation between the fresh stage and other stages (Paczkowski et al., 2012). Only few compounds are emitted in this first stage of decay and, therefore, aldehydes are emitted in high relative abundances (Figure 2). This enables a selective measurement by semi-conductor gas sensor systems. The sensitivity of these gas sensors is sufficient to detect compounds at low concentrations, if they are not mixed with other compounds of higher concentration (Olafsdottir and Kristbergsson, 2006) and methods for further enhancement of gas sensor performances are shown by Sauerwald et al., (Sauerwald et al., 2007) and Gramm et al., (Gramm and Schütze, 2003). Therefore, the aldehydes heptanal and nonanal are further regarded as vital for a volatile based PMI estimation, although they are emitted in low quantities and might drop below the detection limit at low temperatures. 
The reliable quantitative dynamic during the decay is the third criterion evaluated for volatile based PMI estimation. This criterion requires a dynamic emission, which is correlated to the process of decay. Figure 2 displays the relative abundances of the two aldehydes, the two sulfur compounds, butan-1-ol, and phenol. Although C. vicina perceives acetophenone (Table 3 ) this compound is excluded, as its relative emission was found to resemble the aldehyde emission and did not contribute to an improved understanding of the decaying process. Furthermore, it's absolute concentration is low and it is considered to be an oxidation product of the TENAX ${ }^{\circledR}$ powder (DeGreef and Furton, 2011).

The function of aldehydes for the determination of the PMI in the early phase of decay has already been discussed. The sulfur compound emission reliably starts at the onset of the bloated stage. The position of the sampling tubes (head or bloated torso) did not affect the sulfur compound detection, although in case of the $3^{\text {rd }}$ pig the tube was positioned above the head, which was in a progressed stage of decay. The correlation of the sulfur compound emission with the onset of the bloated stage corresponds to observations of Matuszewski et al, 2010 (Matuszewski et al., 2010a), who found an increasing insect activity during the bloated stages of decaying domestic pigs (Sus scrofa domesticus). This was observed in this study, as well. Aak et al (Aak et al., 2010) found that dimethyl trisulfide was attractive for $C$. vicina in a wind tunnel behavior study and Paczkowski et al (Paczkowski et al., 2012) found a very high sensitivity of $C$. vicina towards sulfur compounds. The sulfur compound emission starts at an $A D D_{5}$ of $80\left(1^{\text {st }} \mathrm{pig}\right)$, an $\mathrm{ADD}_{5}$ of $94\left(2^{\text {nd }} \mathrm{pig}\right)$, an $A D D_{5}$ of $85\left(3^{\text {rd }} \mathrm{pig}\right)$, and an $A D D_{5}$ of 121 $\left(4^{\text {th }} \mathrm{pig}\right)$. The higher $A D D_{5}$ value of the $4^{\text {th }}$ pig might be caused by the long time between the last sample of the fresh stage and the first sample of the bloated stage (Figure 2). It is possible that the bloating of the cadaver started earlier than observed. The absolute sulfur compound emission shows a high variation. The $1^{\text {st }}$ pig showed a strong increase of the absolute sulfur compound emission in the middle of the active stage $\left(A D D_{5} 190\right)$, while the $2^{\text {nd }}$ pig shows an increasing absolute concentration at the end of the bloated stage $\left(A_{D D}{ }_{5} 117\right)$. As snails fed on the skin of this pig, the resulting small injuries could have led to an effusion of volatiles from the intestine. This would also explain the high numbers of carrion visiting insects on these injuries. 

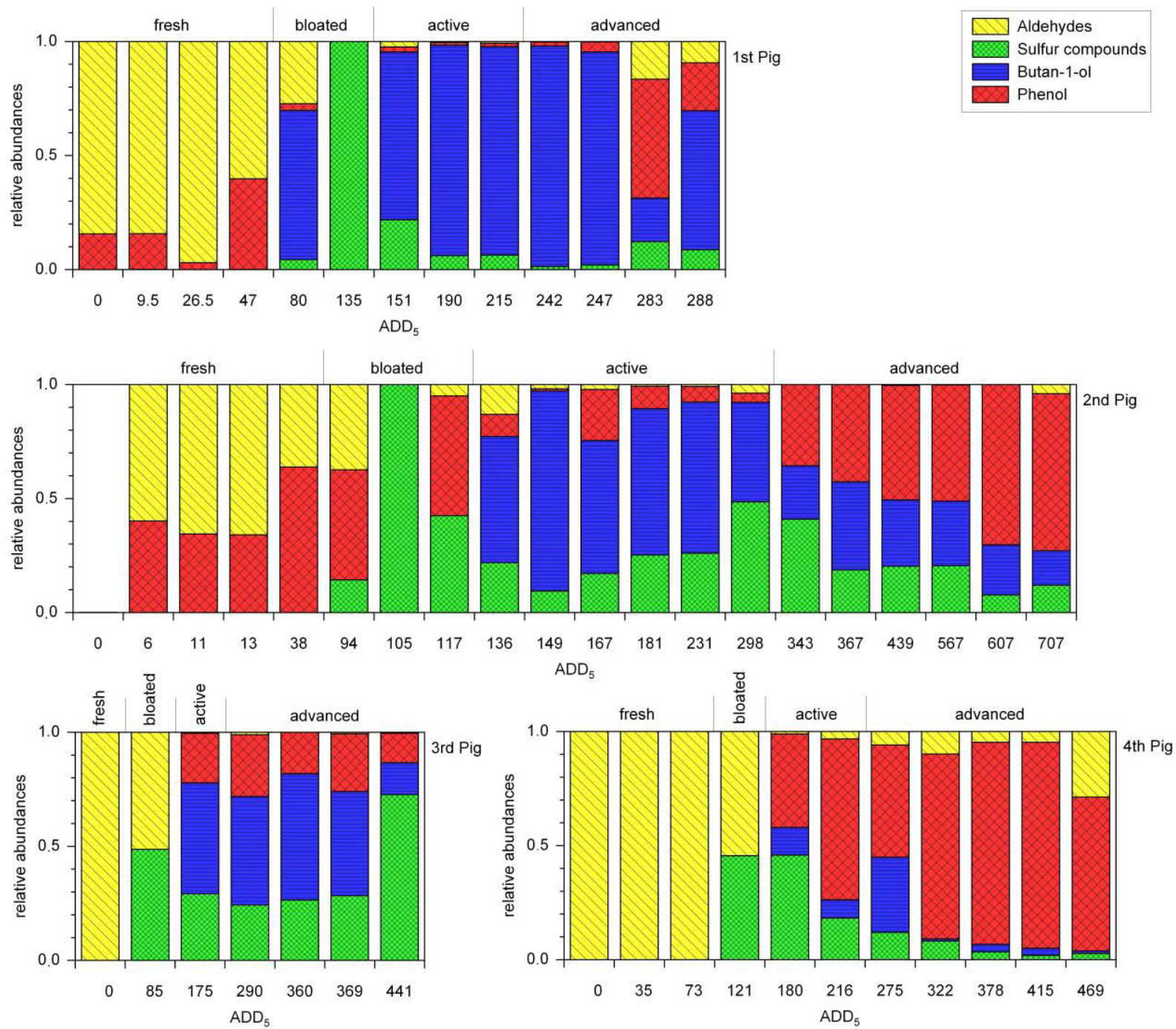

Figure 2: Volatile dynamics. The Figure shows the relative abundance of the aldehydes heptanal and nonanal, the sulfur compounds dimethyl disulfide and dimethyl trisulfide, butan-1-ol, and phenol for the four pigs. The onset and the duration of each stage of decay are shown and the $A_{D D}$ was calculated for each sample in order to evaluate the correlation between optical impression, $\mathrm{ADD}_{5}$ and the relative abundance of the selected volatiles.

The skin breaks and the inner fluid gets in contact with oxygen at the onset of the active stage. This induces a change in the microbial species constitution. Aerobic or facultative aerobic microbes increase their growth rate, while obligate or facultative anaerobic microbes are restricted in their growth. Butan-1-ol is emitted in large quantities, possibly because of a shift in the biochemical pathways of facultative aerobic microbes (Boumba et al., 2008). The compound is detected at the onset of the active stage at an $A D D_{5}$ of 151 ( $\left.1^{\text {st }} \mathrm{pig}\right)$, an $A D D_{5}$ of $136\left(2^{\text {nd }} \mathrm{pig}\right)$, an $A D D_{5}$ of 175 ( $\left.{ }^{\text {rd }} \mathrm{pig}\right)$, and an $\mathrm{ADD}_{5}$ of 180 ( $4^{\text {th }} \mathrm{pig}$ ). The only exception is the bloated stage of the 1 st pig (Table 2). The correlation of butan-1-ol emission to the active stage of decay corresponds to results of Statheropoulos et al., (Statheropoulos et al., 2005), who detected butan-1-ol in the headspace of a corpse with progressed decay and not in the headspace of a corpse in an earlier stage of decay. In the study presented here, the absolute butan-1-ol concentration decreased in the advanced stages of the four pigs (Table 2).

The $1^{\text {st }}$, the $2^{\text {nd }}$ and the $4^{\text {th }}$ pig showed an increasing absolute emission of phenol in the active and advanced stage of decay. Increasing phenol emission might indicate microbial competition for 
decreasing substrates in this late stage of decay (Paczkowski and Schütz, 2011). The $1^{\text {st }}, 2^{\text {nd }}$ and the $4^{\text {th }}$ pig showed a decreasing relative concentration of phenol and an increasing aldehyde concentration in the advanced stage of decay (Figure 2). Increasing aldehyde concentration during the advanced stage of decay has already been suggested in a former study on decaying mouse cadavers (Paczkowski et al., 2012) and by Dekeirsschieter et al., (2009) and could derive from decomposing bones (Vass et al., 2008; Paczkowski and Schütz, 2011) or adipocere formation (Paczkowski and Schütz, 2011). The relative concentration of phenol and aldehydes might give information about the PMI during the advanced stage of decay, although the visible characteristics of the cadaver do not change in this stage. Long term studies have to be performed in order to assess the value of phenol and aldehyde formation for PMI estimation in the later stages of decay.

\section{Conclusion}

C. vicina is a generalist with regard to vertebrate species and stage of decomposition. Its olfaction hints at the aldehydes heptanal and nonanal, and the sulfur compounds dimethyl disulfide and dimethyl trisulfide for a volatile based differentiation between the fresh and the bloated stage of decay. The olfaction of $D$. maculatus, being a specialist with regard to stage of decomposition, reveals additional compounds like butan-1-ol. This compound contributes to a better differentiation between the bloated and the active stage of decay. Although acetophenone and the aldehydes heptanal and nonanal were electrophysiological active on the antenna of $C$. vicina, their role for volatile based PMI estimation was critically discussed. Results of other studies and the restrictions of semi-conductor gas sensors led to the selection of the aldehydes despite their low concentration and the rejection of acetophenone because of its questionable role in the decaying process. This shows that compounds perceived by insects do not necessarily contribute to an improved PMI estimation.

Volatile based PMI estimation shows similar restrictions as established methods and might be of additional value, if the stage of decay cannot be classified or the insect succession is influenced by a high abundance of predators. The relative emission of phenol and aldehydes might give information about the PMI during the advanced stage of decay. Moreover, canine training can be improved by selecting volatile compositions typical for distinct stages of decay. A sensor based on-site method might provide a fast first assessment of the PMI or might improve search operations after natural disasters.

\section{Acknowledgements}

We like to thank August Capelle for selecting the sampling site, building the metal fence cage and organizing the regional contacts with foresters and officials. We like to thank Jörg Berger and Ullrich Aschmann for their technical support. The work of Sebastian Paczkowski was kindly supported by the Cusanuswerk, Bischöfliche Studienstiftung, Germany.

\section{References}

Aak,A., Knudsen,G.K. and Soleng,A. (2010) Wind tunnel behavioural response and field trapping of the blowfly Calliphora vicina. Medical and Veterinary Entomology, 24, 250-257.

Amendt,J., Krettek,R. and Zehner,R. (2004) Forensic entomology. Naturwissenschaften, 91, 51-65. 
Boumba,V.A., Ziavrou,K.S. and Vougiouklakis,T. (2008) Biochemical pathways generating postmortem volatile compounds co-detected during forensic ethanol analyses. Forensic Science International, 174, 133-151.

Carter,D.O., Yellowlees,D. and Tibbett,M. (2007) Cadaver decomposition in terrestrial ecosystems. Naturwissenschaften, 94, 12-24.

Castello,G., Moretti,P. and Vezzani,S. (2009) Retention models for programmed gas chromatography. Journal of Chromatography A, 1216, 1607-1623.

Combet,E., Henderson,J., Eastwood,C.D. and Burton,K.S. (2006) Eight-carbon volatiles in mushrooms and fungi: properties, analysis, and biosynthesis. Mycoscience, 47, 317-326.

Cummings,J.H., Hill,M.J., Bone,E.S., Branch,W.J. and Jenkins,D.J.A. (1979) Effect of meat protein and dietary fiber on colonic function and metabolism .2. Bacterial metabolites in feces and urine. American Journal of Clinical Nutrition, 32, 2094-2101.

DeGreef,L.E. and Furton,K.G. (2011) Collection and identification of human remains volatiles by noncontact, dynamic airflow sampling and SPME-GC/MS using various sorbent materials. Analytical and Bioanalytical Chemistry, 401, 1295-1307.

Dekeirsschieter,J., Verheggen,F.J., Gohy,M., Hubrecht,F., Bourguignon,L., Lognay,G. and Haubruge,E. (2009) Cadaveric volatile organic compounds released by decaying pig carcasses (Sus domesticus L.) in different biotopes. Forensic Science International, 189, 46-53.

Dent,B.B., Forbes,S.L. and Stuart,B.H. (2004) Review of human decomposition processes in soil. Environmental Geology, 45, 576-585.

Derrick,S. and Large,P.J. (1993) Activities of the enzymes of the ehrlich pathway and formation of branched-chain alcohols in saccharomyces-cerevisiae and candida-utilis grown in continuous-culture on valine or ammonium as sole nitrogen-source. Journal of General Microbiology, 139, 2783-2792.

Dourel,L., Pasquerault,T., Gaudry,E. and Vincent,B. (2010) Using estimated on-site ambient temperature has uncertain benefit when estimating postmortem interval. Psyche, 2010, 1-7.

Ercolini,D., Russo,F., Nasi,A., Ferranti,P. and Villani,F. (2009) Mesophilic and psychrotrophic bacteria from meat and their spoilage potential in vitro and in beef. Applied and Environmental Microbiology, 75, 1990-2001.

Evans,W.E.D. (1963) The chemistry of death. Charles C. Thomas Publishers, Springfield, USA.

Geypens,B., Claus,D., Evenepoel,P., Hiele,M., Maes,B., Peeters,M., Rutgeerts,P. and Ghoos,Y. (1997) Influence of dietary protein supplements on the formation of bacterial metabolites in the colon. Gut, 41, 70-76.

Gill-King,H. (1999) Chemical and ultrastructural aspects of decomposition. In Haglund,W.D. and Sorg,M.H. (eds.), Forensic Taphonomy. CRC Press, Boca Raton, USA, pp. 93-108. 
Goll,D.E., Otsuka,Y., Nagainis,P.A., Shannon,J.D., Sathe,S.K. and Muguruma,M. (1983) Role of muscle proteinases in maintenance of muscle integrity and mass. Journal of Food Biochemistry, 7, 137-177.

Gottschalk,G. (1986) Bacterial metabolism. Springer Verlag, New York, USA.

Gramm,A. and Schütze,A. (2003) High performance solvent vapor identification with a two sensor array using temperature cycling and pattern classification. Sensors and Actuators B-Chemical, 95, 5865.

Haze,S., Gozu,Y., Nakamura,S., Kohno,Y., Sawano,K., Ohta,H. and Yamazaki,K. (2001) 2-Nonenal newly found in human body odor tends to increase with aging. Journal of Investigative Dermatology, $116,520-524$

Hoermann,C., Ruther,J., Reibe,S., Madea,B. and Ayasse,M. (2011) The importance of carcass volatiles as attractants for the hide beetle Dermestes maculatus (De Geer). Forensic Science International, 212, 173-179.

Hoffman,E.M., Curran,A.M., Dulgerian,N., Stockham,R.A. and Eckenrode,B.A. (2009) Characterization of the volatile organic compounds present in the headspace of decomposing human remains. Forensic Science International, 186, 6-13.

Huotari,M. and Mela,M. (1996) Blowfly olfactory biosensor's sensitivity and specificity. Sensors and Actuators B-Chemical, 34, 240-244.

Huotari,M.J. (2000) Biosensing by insect olfactory receptor neurons. Sensors and Actuators BChemical, 71, 212-222.

Kaib,M. (1974) Receptors for meat-odor and flower-odor on antenna of blowfly Calliphora-vicina. Journal of Comparative Physiology, 95, 105-121.

Kalinova,B., Podskalska,H., Ruzicka,J. and Hoskovec,M. (2009) Irresistible bouquet of death-how are burying beetles (Coleoptera: Silphidae: Nicrophorus) attracted by carcasses. Naturwissenschaften, 96, 889-899.

LeBlanc,H.N. and Logan,J.G. (2010) Exploiting insect olfaction in forensic entomology. In Amendt,J., Campobasso,C.P., Goff,M.L. and Grassberger,M. (eds.), Current concepts in forensic entomology. Springer, New York, USA, pp. 205-222.

Lorenzo,N., Wan,T.L., Harper,R.J., Hsu,Y.L., Chow,M., Rose,S. and Furton,K.G. (2003) Laboratory and field experiments used to identify Canis lupus var. familiaris active odor signature chemicals from drugs, explosives, and humans. Analytical and Bioanalytical Chemistry, 376, 1212-1224.

Matuszewski,S., Bajerlein,D., Konwerski,S. and Szpila,K. (2010a) Insect succession and carrion decomposition in selected forests of Central Europe. Part 2: Composition and residency patterns of carrion fauna. Forensic Science International, 195, 42-51. 
Matuszewski,S., Bajerlein,D., Konwerski,S. and Szpila,K. (2010b) Insect succession and carrion decomposition in selected forests of Central Europe. Part 1: Pattern and rate of decomposition. Forensic Science International, 194, 85-93.

Michaud,J.P. and Moreau,G. (2011) A statistical approach based on accumulated degree-days to predict decomposition-related processes in forensic studies. Journal of Forensic Sciences, 56, 229-232.

Olafsdottir,G. and Kristbergsson,K. (2006) Electronic-nose technology: Application for quality evaluation in the fish industry. In Nicolay,X. (ed.), Odors in the food industry. Springer, New York USA, pp. 57-74.

O'Neal,C.L. and Poklis,A. (1996) Postmortem production of ethanol and factors that influence interpretation - A critical review. American Journal of Forensic Medicine and Pathology, 17, 8-20.

Paczkowski, S. and Schütz, S. (2011) Post-mortem volatiles of vertebrate decay. Applied Microbiology and Biotechnology 91, 917-935.

Paczkowski, S., Paczkowska, M., Maibaum, F., and Schütz, S. (2012) Decaying mouse volatiles perceived by Calliphora vicina Rob.-Desv. Journal of Forensic Sciences. 57, 1497-1506.

Podskalska,H., Ruziccka,J., Hoskovec,M. and Salek,M. (2009) Use of infochemicals to attract carrion beetles into pitfall traps. Entomologia Experimentalis et Applicata, 132, 59-64.

Sauerwald,T., Skiera,D. and Kohl,C.D. (2007) Selectivity enhancement of gas sensors using nonequilibrium polarisation effects in metal oxide films. Applied Physics A-Materials Science \& Processing, 87, 525-529.

Schütz,S., Weissbecker,B., Koch,U.T. and Hummel,H.E. (1999) Detection of volatiles released by diseased potato tubers using a biosensor on the basis of intact insect antennae. Biosensors and Bioelectronics, 14, 221-228.

Smith,K.G.V. (1986) A manual of forensic entomology. Department of Entomology,British Museum (Natural History), London, United Kingdom.

Statheropoulos,M., Spiliopoulou,C. and Agapiou,A. (2005) A study of volatile organic compounds evolved from the decaying human body. Forensic Science International, 153, 147-155.

Statheropoulos,M., Agapiou,A., Spiliopoulou,C., Pallis,G.C. and Sianos,E. (2007) Environmental aspects of VOCs evolved in the early stages of human decomposition. Science of the Total Environment, 385, 221-227.

Stensmyr,M.C., Urru,I., Collu,I., Celander,M., Hansson,B.S. and Angioy,A.M. (2002) Rotting smell of dead-horse arum florets - These blooms chemically fool flies into pollinating them. Nature, 420, 625626.

Stoffolano,J.G. (1974) Influence of diapause and diet on development of gonads and accessory reproductive glands of black blowfly, Phormia-Regina (Meigen). Canadian Journal of Zoology-Revue Canadienne de Zoologie, 52, 981-991. 
Stutz,H.K., Silverman,G.J., Angelini,P. and Levin,R.E. (1991) Bacteria and volatile compounds associated with ground-beef spoilage. Journal of Food Science, 56, 1147-1153.

Thomson,G. (1946) The antoine equation for vapor-pressure data. Chemical Reviews, 38, 1-39.

Vass,A.A., Barshick,S.A., Sega,G., Caton,J., Skeen,J.T., Love,J.C. and Synstelien,J.A. (2002) Decomposition chemistry of human remains: A new methodology for determining the postmortem interval. Journal of Forensic Sciences, 47, 542-553.

Vass,A.A., Smith,R.R., Thompson,C.V., Burnett,M.N., Wolf,D.A., Synstelien,J.A., Dulgerian,N. and Eckenrode,B.A. (2004) Decompositional odor analysis database. Journal of Forensic Sciences, 49, 760-769.

Vass,A.A., Smith,R.R., Thompson,C.V., Burnett,M.N., Dulgerian,N. and Eckenrode,B.A. (2008) Odor analysis of decomposing buried human remains. Journal of Forensic Sciences, 53, 384-391.

Weissbecker,B., Holighaus,G. and Schütz,S. (2004) Gas chromatography with mass spectrometric and electroantennographic detection: analysis of wood odorants by direct coupling of insect olfaction and mass spectrometry. Journal of Chromatography A, 1056, 209-216.

Zhang,C.H., Hirano,T., Suzuki,T. and Shirai,T. (1992) Studies on the odor of fishes .2. Enzymatically generated specific volatile compounds in ayu tissues. Nippon Suisan Gakkaishi, 58, 559-565. 


\section{The olfaction of a fire beetle leads to new concepts for early fire warning systems}

\section{Authors names and affiliations:}

Corresponding author: Sebastian Paczkowski ${ }^{\mathrm{a} 1}$, Dept. of Forest Zoology and Forest Conservation, Büsgen-Institute, Georg-August-University, Göttingen, Germany. email: SebastianPaczkowski@gmx.de, Tel.: +49-07666-6026417, fax: fax: +49-551-39-22089

Marta Paczkowska a , Dept. of Forest Zoology and Forest Conservation, Büsgen-Institute, GeorgAugust-University, Göttingen, Germany. email: mpaczko@gwdg.de

Stefan Dippel ${ }^{b}$, Dept. of Developmental Biology, Büsgen-Institute and Johann-Friedrich-BlumenbachInstitute of Zoology and Anthropology, Georg-August-University, Göttingen, Germany, email: dpl13@gmx.de

Norman Schulze ${ }^{a}$, Dept. of Forest Zoology and Forest Conservation, Büsgen-Institute, Georg-AugustUniversity, Göttingen, Germany. email: schulzenorman@googlemail.com

Prof. Dr. Stefan Schütz ${ }^{\mathrm{a}}$, Dept. of Forest Zoology and Forest Conservation, Büsgen-Institute, GeorgAugust-University, Göttingen, Germany, email: stefan.schuetz@forst.uni-goettingen.de

Dr. Tilman Sauerwald ${ }^{c}$, Institute of Measurement Techniques, University of Saarland, Saarbrücken, Germany, email: t.sauerwald@Imt.uni-saarland.de

Alexander Weiß ${ }^{d}$, Institute of Applied Physics, Justus-Liebig-University, Gießen, Germany, email: Alexander.Weiss@ap.physik.uni-giessen.de

Marco Bauer ${ }^{d}$, Institute of Applied Physics, Justus-Liebig-University, Gießen, Germany, email: Marco.Bauer@ap.physik.uni-giessen.de

Dr. Jörg Gottschald ${ }^{d}$, Institute of Applied Physics, Justus-Liebig-University, Gießen, Germany, email: Joerg.Gottschald@ap.physik.uni-giessen.de

Prof. Dr. Dieter Kohl ${ }^{d}$, Institute of Applied Physics, Justus-Liebig-University, Gießen, Germany, email: Dieter.Kohl@ap.physik.uni-giessen.de

Running head: Beetle olfaction for early fire warning

\footnotetext{
${ }^{1}$ Permanent address: Schwarzwaldstr. 48, 79211 Denzlingen, Germany
} 


\begin{abstract}
The European fire beetle Melanophila cuspidata oviposits on heated and superficially charred stems to provide its xylophagous larvae with nutrition. We investigated, if the beetle smells marker volatiles allowing an assessment of the heating stage of the wood beneath the bark. Such biologically preselected marker volatiles will enable a reliable differentiation between fresh, heated, and burned wood and will be useful for early fire warning systems. 25 compounds of heated pine wood chips (Pinus sylvestris) were identified and quantified by GC-MS. Electrophysiological experiments (GCMS/EAD, EAG) and behavioral studies with $M$. cuspidata revealed eight electrophysiologically active and behaviorally attractive compounds. From these compounds furfural was selected as a marker volatile for heated wood from these compounds. A nanoporous semiconductor metal oxide gas sensor was developed for the detection of furfural. This sensor detected furfural sensitively in a burning chamber prior to the ignition of pine wood chips ( $P$. sylvestris). The furfural emission in this chamber was monitored simultaneously by an online mass-spectrometer. The utilization of $M$. cuspidata for the selection of marker volatiles and the feasibility of the nanoporous sensor for early fire warning systems in the wood processing industry is discussed.
\end{abstract}

Keywords: (Melanophila cuspidata, thermal oxidation, wood, furfural, nanoporous semiconductor metal oxide gas sensor, biomimetik sensor)

\title{
Introduction
}

Wood is a complex tissue consisting of the structural polymers hemicelluloses, cellulose, lignin, and a broad variety of accessory compounds. The relative amount of these constituents varies between tree species, within tree species and even within one tree (Kollmann, 1982). Therefore, the qualitative and quantitative volatile pattern emitted during the thermal oxidation of wood is also complex. One or several reliably occurring combustion products of wood have to be selected in order to develop an early fire warning system for wood. Such combustion products, further referred to as marker volatiles, should have three characteristics. Firstly, marker volatiles should be emitted reliably by any kind of heated wood and should occur reproducibly prior to ignition. This will ensure that an early fire warning system will not fail to detect an increasing fire risk. Secondly, the marker volatiles should be emitted at relatively high concentration. Semiconductor gas sensors are very sensitive, but a volatile compound at low concentration might not be detectable in a volatile mixture, since such gas sensors are not very selective. Thirdly, marker volatiles should be characteristic for the thermal oxidation or the pyrolysis of wood and should not be emitted by other sources in order to reduce false alarms. The selection of such marker volatiles will require many experiments, because the variation among volatile combustion products of wood is very high.

\section{Biomimetik approach}

A more efficient option to select such marker volatiles is the utilization of the pyrophilic beetle Melanophila cuspidata (Buprestidae; Klug, 1829). Its olfaction is optimized to perceive volatile compounds emitted by forest fires (Schütz et al., 1999). The beetles mate, and the female beetles oviposit, on stems with heated but unburned wood tissue on recently burned areas. Such stems are not able to defend themselves any more, but still provide nutrition for the larvae. The beetles have to distinguish between intact, heated, and burned wood beneath the bark in order to find the ideal 
oviposition place. This cannot be done by the optical impression of the stems, because all stems are charred after a fire. The infrared organ of the beetle (Schmitz et al., 1997; Kreiss et al., 2007) might be able to differentiate between cold, warm, and hot stems to avoid the landing on hot patches; however, this information does not allow the estimation of the physiological stage of the stem beneath its charred bark. The physiological stage can be estimated by the absence or presence of volatile compounds that form during the thermal oxidation of wood. Volatiles smelled by the beetles can be reliable markers for the heating stages of wood, because the survival of their offspring depends on a correct assessment of these stages. The perceived volatiles will have a high potential for fulfilling the first criterion for the proposed marker volatiles. Therefore, the chemoecology of $M$. cuspidata can preselect potential marker volatiles for a reliable semiconductor gas sensor system.

\section{Requirements of biomimetik metal oxide gas sensors}

Such a semiconductor gas sensor should exhibit a long life time, operate at low power conditions, and should have the potential for mass production. The sensor should be sensitive enough to detect marker volatiles at low concentration and selective enough to minimize the influence of other gases. Semiconductor metal oxide gas sensors have most of these characteristics. They have the potential for low power consumption applications (Gutmacher et al., 2011) and temperature cycles can improve their selectivity (Semancik et al., 2001, Gramm and Schütze, 2003). Nanoporous semiconductor metal oxide gas sensors exhibit an improved sensitivity towards target volatiles in comparison to bulk material sensors (Kakati et al., 2009, Paczkowski et al., 2011). An ordered mesoporous tungsten oxide gas sensors made by nanocasting was used for the selective and sensitive detection of furfural, which is a compound utilized by M. cuspidata, and run with a temperature cycle. The performance of this biomimetic sensor in differentiating between stages of heated pine wood (Pinus sylvestris, Coniferalis: Pinaceae; Linnaeus, 1753) was evaluated in a burning chamber.

\section{Material and Methods}

\section{Thermal oxidation of wood chips, cellulose and lignin}

A modified GC oven (Fractovap Series 4160, Carlo Erba Strumentazione, Rodano, Italy) was used as a heating chamber for the thermal oxidation experiments. A glass flask (250 ml Duran, Schott AG, Mainz, Germany) was put into the oven. Synthetic air $\left(20 \% \mathrm{O}_{2}\right.$ in $\mathrm{N}_{2}$, Alpha Gaz, Düsseldorf, Germany) was lead into the flask with 1 bar pressure. The air was sucked out of the flask by a rotary vane pump (1.2 l/min, Thomas Division, Sheboygan, USA) and was led through a charcoal trap (1.8 mg Charcoal, CLSA Filter, Daumazan sur Arize, France) for collecting volatiles. A control sample of 0.6 I air from a clean flask was taken at $250^{\circ} \mathrm{C}$. Afterwards, the xylem of a fresh branch $(\varnothing=2.5-3 \mathrm{~cm})$ of $P$. sylvestris was drilled with a $5 \mathrm{~mm}$ diameter wood driller and $2 \mathrm{~g}$ of the chips were inserted into the glass flask. After $10 \mathrm{~min}$ conditioning time at $25{ }^{\circ} \mathrm{C}$ the rotary vane pump sucked $0.6 \mathrm{~L}$ through the charcoal filter tube. This procedure was repeated for the temperature steps $50^{\circ} \mathrm{C}, 75^{\circ} \mathrm{C}, 100^{\circ} \mathrm{C}, 150$ ${ }^{\circ} \mathrm{C}, 200{ }^{\circ} \mathrm{C}, 250{ }^{\circ} \mathrm{C}$ and $300{ }^{\circ} \mathrm{C}$ in order to collect the volatiles of fresh, heated, and burning wood chips. Two gram of cellulose powder (DS-0, Sigma-Aldrich, St. Louis, USA) or lignin powder (KraftLignin, Sigma-Aldrich, St. Louis, USA) were filled into a $4 \mathrm{ml}$ glass vial with a perforated aluminum cap in order to evaluate the origin of the wood chips' emissions. The vials were put into the glass flask 
and the volatile sampling was performed in the same way as described. The described sampling procedures were repeated five times with the wood chips, the cellulose powder, and the lignin powder.

\section{Trace analysis of the volatile samples}

The trace analysis was performed according to a method described in Paczkowski et al., 2012 (p.71).

\section{Quantification of volatiles from thermally oxidized wood flakes}

The standard solutions for the confirmation of the retention time were used for quantification. The main ion of each compound was extracted and quantified using the Enhanced Chemstation Version D00.00.38 software from Agilent Technologies (Santa Clara, USA). The peak area of the main ion in the standard solution resembled $\sim 100 \mathrm{ng}$. The quantity in the samples was calculated in ppmv and each data set was tested for normal distribution with the Shapiro-Wilk Test. Most of the datasets were not normally distributed, therefore, the data was described by the median, the lower and upper quartiles, and minima and maxima (Table 2 and 3). A minimum of 0 indicates that the compound is not present in all of the five replications.

\section{Insect collection and rearing}

Wood stems ( $P$. sylvestris) containing larvae of Melanophila cuspidata were collected on a burned woodland area in Sallent - Balsareny, Spain, in March 2010. They were transported to Germany and incubated at $37^{\circ} \mathrm{C} \pm 1{ }^{\circ} \mathrm{C}, 40 \% \pm 10 \%$ relative humidity and a $12 \mathrm{~h} / 12 \mathrm{~h}$ day/night cycle. The adult beetles hatched $16 \pm 2$ days later and were collected for electrophysiological and behavioral experiments.

\section{GC-MS/EAD with $M$. cuspidata}

GC-MS/EAD studies were performed as described in Weissbecker et al., 2004. The volatile samples of three selected thermal oxidation experiments of wood chips were used for the GC-MS/EAD studies. Each sample was tested on three beetle antennae and only compounds repeatedly eliciting antennal responses were selected for further EAG experiments.

\section{EAG with M. cuspidata}

The EAG was performed according to a method described in detail in Paczkowski et al., 2012 (p.72). Furthermore, the vapor pressure of the $1^{\text {st }}$ significant concentration of the beetles towards the tested compounds was calculated with Henrie's law as the vapor pressure of the compound's dilution at which the beetles showed the first significant response.

\section{Behavioral studies with M. cuspidata}

All compounds which were selected for the EAG experiments were tested in paraffin oil dilutions of $10^{-2}$ and $10^{-4}(\mathrm{~g} / \mathrm{g})$ in the behavioral studies. Additionally, a volatile mixture of 2-methoxyphenol and 5-methylfurfural (1:1) diluted to $10^{-4}(\mathrm{~g} / \mathrm{g})$ in paraffin oil was tested. A custom built two arm

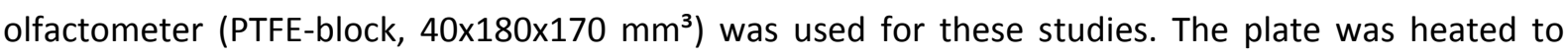
$30 \pm 2{ }^{\circ} \mathrm{C}$ by a heating plate beneath the olfactometer. Ten parallel walking chambers $\left(20 \times 8 \times 150 \mathrm{~mm}^{3}\right)$ were covered with an acryl glass lid. A comb with metal tubes confined the beetles in the middle of the walking chambers. $100 \mu \mathrm{l}$ filter pipette tips (Sarstedt AG\&CO, Nümbrecht, Germany) were inserted from both sides of each walking chamber. Each filter contained two drops of either the odor 
dilution or pure paraffin oil or none of the compounds for the choice tests with pure air. Charcoal filtered and humidified air $\left(21 \pm 1{ }^{\circ} \mathrm{C}, 40 \%\right.$ relative humidity) was led through the walking chambers at a constant flow of $0.65 \mathrm{~L} / \mathrm{min}$. The beetles were able to move for seven minutes after the comb was removed. The walking chambers were scaled into 500 single positions. The movement of the beetles was recorded by a camera (iSlim, Kye Systems Corp.) and captured by a picture converter (VirtualDub 1.9.10, A. Lee, Freeware, 2 pictures / s). The position of each beetle in the olfactometer was calculated with the program ImageJ $1.43 \mathrm{u}$ (National Institute of Health, USA) and the plugin MTrack2 (National Institute of Health, USA) for every second. The attractivity index of each stimulus was calculated, expressing the mean value of the beetle position as the percentage of the theoretical maximum mean value. The attractivity index was $100 \%$ in case of a maximum attraction, $50 \%$ in case of indifferent behavior and $0 \%$ in case of a maximum repellence. Significant difference between stimulus and paraffin oil was tested with the Mann-Whitney U-Test for skew distributed data sets. A $p$-level of $5 \%$ was the threshold for significance.

\section{Sensor preparation and performance in a gas mixing equipment}

The sensors were prepared by depositing a suspension of $\mathrm{WO}_{3}$ on a commercial alumina substrate with an interdigitated platinum electrode (Umweltsensortechnik $\mathrm{GmbH}$, Geschwenda, Germany). Nanoporous material obtained by a nanocasting process was compared with bulk material (Fluka, St. Gallen, Switzerland). The details of the $\mathrm{WO}_{3}$ nanocasting process are described in Eßinger et al., 2010 and based on a method of Rossinyol et al., (Rossinyol and Tian et al., 2005). The sensor temperature was controlled by integrated platinum wires.

The gas sensing properties were measured by means of a gas-mixing equipment (Multi Gas Controller 647B, MKS Instruments Deutschland GmbH, München, Germany). Standard mass-flow controllers (1179A Mass-Flo Controller, MKS Instruments Deutschland GmbH, München, Germany) were used to generate two well defined synthetic air flows. One gas flow was led through a custom build dewar, which was filled with furfural. At a constant temperature of $-5{ }^{\circ} \mathrm{C}$, generated with a Cryostat (RM 6, Messgeräte-Werke Lauda Dr. R. Wobser GmbH \& CO. KG, Lauda-Königshofen, Germany), a constant vapor pressure and, therefore, a constant concentration of $140 \mathrm{ppmv}$ above the liquid furfural was generated. In combination with the second synthetic air flow arbitrary concentrations between 0 und 140 ppmv of furfural were adjusted.

Changes in sensor conductance were measured at a constant voltage of $0.5 \mathrm{~V}$. The sensor response is defined as the relative change in conductance $\left(\left(G-G_{0}\right) / G_{0}\right)$ where $G$ is the sensor response towards furfural and $G_{0}$ is the sensor response towards pure synthetic air.

\section{Fire experiments in a burning chamber}

$\sim 20 \mathrm{~g}$ of dried OSB strands (Pinus sylvestris) were heated in a $50 \times 50 \times 60 \mathrm{~cm}^{3}$ burning chamber (150 L). An infrared-heater with an integrated thermocouple was used in order to adjust the required temperature. The wood chips were placed on the surface of the IR-heater and were heated from 25 ${ }^{\circ} \mathrm{C}$ to $50{ }^{\circ} \mathrm{C}, 100{ }^{\circ} \mathrm{C}, 150{ }^{\circ} \mathrm{C}, 200{ }^{\circ} \mathrm{C}, 250{ }^{\circ} \mathrm{C}, 300{ }^{\circ} \mathrm{C}, 350{ }^{\circ} \mathrm{C}$, and $400{ }^{\circ} \mathrm{C}$. The temperature was kept constant for $15 \mathrm{~min}$ at each step and the volatile emissions of the wood chips were detected by the described nanoporous metal oxide semiconductor gas sensor. A temperature variation technique was used in order to improve the sensors selectivity (Semancik et al., 2001, Gramm et al., 2003). The surface temperature of the sensor was raised in a triangular shaped cycle with a duration of $20 \mathrm{~s}$ 
(minimum $=250{ }^{\circ} \mathrm{C}$; maximum $=500{ }^{\circ} \mathrm{C}$ ), while acquiring 200 equidistant data points for each cycle (see inset Figure 3). Two features were defined for the discrimination of the temperature stages in the fire experiment. An explorative data analysis was applied using the conductivity in point $X$ and $Y$ in the temperature cycle (see inset Figure 3). Significant differences between the temperature steps were evaluated with the sign test. A $p$-level of $5 \%$ was the threshold for significance.

\section{Volatile detection in a burning chamber by an online quadrupole mass spectrometer}

The gases from the burning chamber were sucked through the capillary inlet of an online quadrupole mass spectrometer (Cirrus 300, MKS Instruments, Inc.) with a rate of $20 \mathrm{ml} / \mathrm{min}$. The inlet was heated to $150{ }^{\circ} \mathrm{C}$ in order to avoid the condensation of volatiles. The mass spectrometer was working in the peak jump mode with an electron energy of $70 \mathrm{eV}$ and detected the amount of the mass $\mathrm{m} / \mathrm{z}=$ 96 in parallel to the nanoporous metal oxide gas sensor. This is the most abundant mass in the spectra of furfural ionized at $70 \mathrm{eV}$. The recording of this mass was used to monitor the emission of furfural during the heating of the wood chips and, thereby, enable the evaluation of the sensor performance.

\section{Results}

\section{Trace analysis and quantification of the wood flake volatiles}

Twenty-five compounds were identified in the volatile samples of the thermally oxidized wood flakes (Table 1) and the quantitative dynamics of the compounds are shown in Table 2. N,Ndimethylformamide is emitted at constant emission rates throughout the heating process, but never in all five tested samples (minimum=0). All of the other compounds are emitted in specific temperature ranges. The first group of compounds consists of the terpenes $\alpha$-pinene, $\beta$-pinene and 3-carene; the alcohols 1-octen-3-ol, 1-octanol, and 1-pentanol; the aldehydes hexanal, heptanal, nonanal, and decanal; and the furane 2-pentylfurane. The volatiles of this group are emitted in the temperature range between $25{ }^{\circ} \mathrm{C}$ (fresh stage) and $250{ }^{\circ} \mathrm{C}$ (Prefiring stage). The second group of compounds consists of the furanes furfural, 5-methylfurfural, 2-furanmethanol, and 5hydroxymethylfurfural; the ketone hydroxyacetone; the acid acetic acid; and the methoxyphenol 6formyl-2-methoxyphenol. These compounds are emitted in the temperature range from $200{ }^{\circ} \mathrm{C}$ (Heating stage) to $300{ }^{\circ} \mathrm{C}$ (Post-ignition stage). The third group of volatiles is emitted in the temperature range from $250{ }^{\circ} \mathrm{C}$ (Prefiring stage) to $300{ }^{\circ} \mathrm{C}$ (Post-ignition stage). This group comprises the methoxyphenols 4-ethyl-2-methoxyphenol, 2-methoxyphenol, 2-methylphenol and 4methylphenol, and the furane 2(5)furanone. Most of the compounds are emitted at a median concentration in the single digit ppmv range per $30 \mathrm{~s}$. Acetic acid, hydroxyaceton, hexanal, N,Ndimethylformamide, furfural, $2(5 \mathrm{H})$-furanone, and $\alpha$-pinene are emitted at or above a median concentration in the double digit ppmv range. Among this group, only hexanal, furfural, $2(5) \mathrm{H}-$ furanone, and $\alpha$-pinene are emitted in all of the five samples of one or several temperature steps (minimum $>0$ ). At $250^{\circ} \mathrm{C}$ (Prefiring stage), furfural is reliably emitted and shows the highest minimum of all compounds and all temperature steps.

The thermal oxidation of cellulose powder and lignin powder resulted in less volatile compounds than the thermal oxidation of the wood chips (Table 3). All of the compounds quantified were 
reliably present (minimum>0), except 4-ethyl-2-methoxyphenol at $200{ }^{\circ} \mathrm{C}$. Levoglucosenone showed large peak areas in the samples at $250{ }^{\circ} \mathrm{C}$ and $300{ }^{\circ} \mathrm{C}$ during the thermal oxidation of cellulose, but the compound was not quantified as it did not occur during the thermal oxidation of the wood flakes. Four other compounds were not identified in the cellulose powder experiments and did not occur in the wood chips experiments. Lignin powder emitted 2-methoxyphenol at and above $150{ }^{\circ} \mathrm{C}$. The chemical pretreatment of the powder (kraft pulping) could explain the presence of 2-methoxyphenol at this early temperature step. Phenol $\left(150{ }^{\circ} \mathrm{C}-300^{\circ} \mathrm{C}\right)$ and 4-propyl-2-methoxyphenol $\left(250{ }^{\circ} \mathrm{C}-300\right.$ ${ }^{\circ} \mathrm{C}$ ) were emitted during the thermal oxidation of the lignin powder and not in the wood chips experiments. Neither cellulose powder nor lignin powder did ignite in any of the repetitions. Cellulose showed a brown color at $300^{\circ} \mathrm{C}$.

Table 1 lists the compounds identified in samples of heated wood flakes (Pinus sylvestris) in a temperature range from $25^{\circ} \mathrm{C}$ to $300^{\circ} \mathrm{C}$.

\begin{tabular}{|c|c|c|c|c|c|c|c|c|}
\hline No. & Compound & $\begin{array}{c}\text { HP-5MS } \\
\text { (LRI) }\end{array}$ & $\begin{array}{l}\text { INNOWAX } \\
\text { (LRI) }\end{array}$ & $\begin{array}{l}\text { Chemical } \\
\text { class }\end{array}$ & CAS No. & Company & $\begin{array}{c}\text { Purity } \\
\text { [\%] }\end{array}$ & $\begin{array}{l}\text { Ion for } \\
\text { quanti- } \\
\text { fication } \\
{[\mathrm{m} / \mathrm{z}]}\end{array}$ \\
\hline 1 & Acetic acid & $<800$ & 1364 & Acid & $64-19-7$ & Fluka & 99 & 43 \\
\hline 2 & Hydroxyacetone & $<800$ & 1195 & Ketone & $116-09-6$ & Aldrich & 98 & 43 \\
\hline 3 & 1-Pentanol & 810 & 1166 & Alcohol & $71-41-0$ & Aldrich & 99 & 42 \\
\hline 4 & Hexanal & 826 & 1100 & Aldehyde & $66-25-1$ & Aldrich & 98 & 44 \\
\hline 5 & $\mathrm{~N}, \mathrm{~N}$-Dimethylformamide & 827 & 1242 & Aldehyde & $68-12-2$ & Aldrich & 98 & 73 \\
\hline 6 & Furfural & 860 & 1357 & Furane & $98-01-1$ & Acros & 99 & 96 \\
\hline 7 & 2-Furanmethanol & 877 & 1564 & Furane & $98-00-0$ & Merck & 98 & 98 \\
\hline 8 & Heptanal & 910 & 1145 & Aldehyde & 111-71-7 & Aldrich & 95 & 44 \\
\hline 9 & 2(5H)-Furanone & 924 & 1602 & Ketone & $497-23-4$ & Aldrich & 98 & 55 \\
\hline 10 & $\alpha$-Pinene & 937 & 1035 & Terpene & $80-56-8$ & Merck & 98 & 93 \\
\hline 11 & $\beta$-Pinene & 980 & 1109 & Terpene & $127-91-3$ & Fluka & 98 & 93 \\
\hline 12 & 5-Methylfurfural & 985 & 1499 & Furane & $620-02-0$ & Across & 98 & 110 \\
\hline 13 & 1-Octen-3-ol & 989 & 1379 & Alcohol & 3391-86- & Merck & 99 & 57 \\
\hline 14 & 2-Pentylfurane & 1002 & 1161 & Furane & $3777-69-$ & $A B C R$ & 98 & 81 \\
\hline 15 & 3-Carene & 1013 & 1124 & Terpene & $498-15-7$ & Fluka & 99 & 93 \\
\hline 16 & 2-Methylphenol & 1067 & 1921 & Phenol & $95-48-7$ & Aldrich & 98 & 108 \\
\hline 17 & 1-Octanol & 1084 & 1490 & Alcohol & $111-87-5$ & Aldrich & 98 & 56 \\
\hline 18 & 4-Methylphenol & 1094 & 1999 & Phenol & $106-44-5$ & Aldrich & 99 & 107 \\
\hline 19 & 2-Methoxyphenol & 1105 & 1777 & Methoxyphenol & $90-05-1$ & Fluka & 98 & 109 \\
\hline 20 & Nonanal & 1118 & 1344 & Aldehyde & $124-19-6$ & Merck & 98 & 57 \\
\hline 21 & 4-Methyl-2-methoxyphenol & 1202 & 1883 & Methoxyphenol & $93-51-6$ & Aldrich & 98 & 138 \\
\hline 22 & Decanal & 1219 & 1437 & Aldehyde & $112-31-2$ & Acros & 95 & 43 \\
\hline 23 & 5-Hydroxymethylfurfural & 1245 & 2348 & Furane & $67-47-0$ & Aldrich & 95 & 97 \\
\hline 24 & 4-Ethyl-2-methoxyphenol & 1296 & 1958 & Methoxyphenol & 2785-89- & SAFC & 98 & 137 \\
\hline 25 & 6-Formyl-2-methoxyphenol & 1421 & 2397 & Methoxyphenol & $148-53-8$ & Aldrich & 98 & 152 \\
\hline
\end{tabular}


Table 2: Emission of wood flakes. The table shows the temperature dependent emission rate (ppmv in $30 \mathrm{~s}$ ) of the identified compounds in the volatile samples of $2 \mathrm{~g}$ heated wood flakes (Pinus sylvestris). The values of the emission rate are displayed in the following order: median, lower quartile, upper quartile, minima, and maxima. N.d. means not detected. A minimum above $0 \mathrm{ppmv}$ in $30 \mathrm{~s}$ ) shows a reliable emission of the compound at this temperature step.

\begin{tabular}{|c|c|c|c|c|c|c|c|c|}
\hline \multirow[t]{3}{*}{ Compound } & \multicolumn{8}{|c|}{ Emission rate [ppmv in 30s] } \\
\hline & $25^{\circ} \mathrm{C}$ & $50^{\circ} \mathrm{C}$ & $75^{\circ} \mathrm{C}$ & $100^{\circ} \mathrm{C}$ & $150^{\circ} \mathrm{C}$ & $200^{\circ} \mathrm{C}$ & $250^{\circ} \mathrm{C}$ & $300^{\circ} \mathrm{C}$ \\
\hline & \multicolumn{5}{|c|}{ Fresh stage } & Heating stage & Prefiring stage & Post-Ignition \\
\hline \multicolumn{9}{|l|}{ Wood flakes (Pinus sylvestris) } \\
\hline Acetic acid & n.d. & n.d. & n.d. & n.d. & n.d. & $18,16,32,0,51$ & $268,245,411,0,654$ & $52,20,73,0,664$ \\
\hline Hydroxyacetone & n.d. & n.d. & n.d. & n.d. & n.d. & $5,4,6,0,8$ & $41,33,41,0,75$ & $14,10,29,0,38$ \\
\hline 1-Pentanol & n.d. & n.d. & n.d. & n.d. & $4,1,10,0,13$ & $4,3,7,0,7$ & $2,1,3,0,5$ & n.d. \\
\hline Hexanal & n.d. & n.d. & n.d. & n.d. & $14,4,29,3,69$ & $18,9,20,8,25$ & $10,7,11,5,13$ & n.d. \\
\hline $\mathrm{N}, \mathrm{N}$-Dimethylformamide & $18,17,22,0,42$ & $5,5,5,0,8$ & $13,12,16,0,24$ & $13,12,19,0,26$ & $15,12,15,0,32$ & $15,14,20,0,23$ & $21,17,22,0,26$ & $15,9,17,0,37$ \\
\hline Furfural & n.d. & n.d. & n.d. & n.d. & n.d. & $4,3,6,3,8$ & $64,58,92,56,98$ & $1,1,2,1,96$ \\
\hline 2-Furanmethanol & n.d. & n.d. & n.d. & n.d. & n.d. & $0,0,0,0,1$ & $3,2,3,2,3$ & $1,1,1,1,2$ \\
\hline Heptanal & n.d. & n.d. & n.d. & n.d. & $0.2,0,1,0,1$ & $1,0.6,1,0.2,2$ & $0.4,0.4,1,0.2,1$ & n.d. \\
\hline 2(5H)-Furanone & n.d. & n.d. & n.d. & n.d. & n.d. & $0,0,0,0,0.3$ & $13,12,21,8,23$ & $16,6,17,2,40$ \\
\hline$\alpha$-Pinene & $16,11,21,3,166$ & $2,1,3,1,5$ & $2,1,5,0.5,7$ & $1,0.2,1,0.2,4$ & $0.2,0.2,0.2,0,3$ & $0,0,0,0,0.8$ & n.d. & n.d. \\
\hline$\beta$-Pinene & $0.3,0.3,12,0.2,62$ & $0,0,0.2,0,9$ & $0,0,0,0,18$ & $0,0,0,0,9$ & $0,0,0,0,8$ & $0,0,0,0,2$ & n.d. & n.d. \\
\hline 5-Methylfurfural & n.d. & n.d. & n.d. & n.d. & n.d. & $0,0,0.2,0,0.2$ & $2,1,3,2,3$ & $1,0.2,1,0,7$ \\
\hline 1-Octen-3-ol & n.d. & n.d. & n.d. & n.d. & n.d. & $0.4,0.2,0.7,0.2,1$ & n.d. & n.d. \\
\hline 2-Pentylfuran & n.d. & n.d. & n.d. & n.d. & $1,0.5,1,0.2,4$ & $1,1,1,0.6,2$ & $0.2,0.2,0.3,0.2,0.5$ & n.d. \\
\hline 3-Carene & $6,4,14,3,113$ & $1,1,2,1,2$ & $1,0.3,2,0.2,5$ & $0.2,0,0.3,0,2$ & $0,0,0.2,0,2$ & $0,0,0,0,1$ & n.d. & n.d. \\
\hline 2-Methylphenol & n.d. & n.d. & n.d. & n.d. & n.d. & n.d. & $0,0,0,0,0.2$ & $1,0.2,2,0.2,4$ \\
\hline 1-Octanol & n.d. & n.d. & n.d. & n.d. & n.d. & $0.4,0.2,1,0.2,1$ & n.d. & n.d. \\
\hline 4-Methylphenol & n.d. & n.d. & n.d. & n.d. & n.d. & n.d. & $0,0,0.2,0,0.2$ & $0.4,0.4,1,0,2$ \\
\hline 2-Methoxyphenol & n.d. & n.d. & n.d. & n.d. & n.d. & n.d. & $2,1,2,1,3$ & $7,2,8,0.3,9$ \\
\hline Nonanal & n.d. & n.d. & n.d. & n.d. & $0.3,0.3,1,0.2,2$ & $2,1,3,1,3$ & $2,1,3,1,5$ & n.d. \\
\hline 4-Methyl-2-methoxyphenol & n.d. & n.d. & n.d. & n.d. & n.d. & n.d. & $1,1,2,1,3$ & $5,4,9,1,13$ \\
\hline Decanal & n.d. & n.d. & n.d. & n.d. & n.d. & $0.2,0.2,0.3,0.2,0.3$ & $0.6,0.3,0.6,0,1$ & n.d. \\
\hline 5-Hydroxymethylfurfural & n.d. & n.d. & n.d. & n.d. & n.d. & $2,2,3,2,3$ & $5,3,7,2,12$ & $5,2,47,1,99$ \\
\hline 4-Ethyl-2-methoxyphenol & n.d. & n.d. & n.d. & n.d. & n.d. & n.d. & $0.2,0.2,0.3,0.2,0.3$ & $0.8,0.6,1,0.3,1$ \\
\hline 6-Formyl-2-methoxyphenol & n.d. & n.d. & n.d. & n.d. & n.d. & $0.3,0.2,0.5,0,0.6$ & $10,6,13,4,26$ & $4,2,27,1,36$ \\
\hline
\end{tabular}


Table 3: Emission of cellulose and lignin. The Table shows the temperature dependent emission rate (ppmv in $30 \mathrm{~s}$ ) of the identified compounds in the volatile samples of cellulose powder $(2 \mathrm{~g})$ and lignin powder $(2 \mathrm{~g})$. The values of the emission rate are displayed in the following order: median, lower quartile, upper quartile, minima, and maxima. N.d. means not detected. A minimum above $\mathbf{0}$ ppmv in $\mathbf{3 0}$ s shows a reliable emission of the compound at this temperature step.

\begin{tabular}{|c|c|c|c|c|c|c|c|c|}
\hline \multirow[t]{3}{*}{ Compound } & \multicolumn{8}{|c|}{ Emission rate [ppmv in 30s] } \\
\hline & $25^{\circ} \mathrm{C}$ & $50^{\circ} \mathrm{C}$ & $75^{\circ} \mathrm{C}$ & $100^{\circ} \mathrm{C}$ & $150^{\circ} \mathrm{C}$ & $200^{\circ} \mathrm{C}$ & $250^{\circ} \mathrm{C}$ & $300^{\circ} \mathrm{C}$ \\
\hline & \multicolumn{6}{|c|}{ Fresh stage } & \multicolumn{2}{|c|}{ Heating stage } \\
\hline \multicolumn{9}{|l|}{ Cellulose } \\
\hline Furfural & n.d. & n.d. & n.d. & n.d. & n.d. & n.d. & $7,6,9,5,11$ & $141,140,180,87,181$ \\
\hline 5-Methylfurfural & n.d. & n.d. & n.d. & n.d. & n.d. & n.d. & n.d. & $11,10,12,7,13$ \\
\hline $\begin{array}{l}\text { 5-Hydroxy- } \\
\text { methylfurfural }\end{array}$ & n.d. & n.d. & n.d. & n.d. & n.d. & n.d. & n.d. & $6,3,10,3,12$ \\
\hline \multicolumn{9}{|l|}{ Lignin } \\
\hline 2-Methoxyphenol & n.d. & n.d. & n.d. & n.d. & $4,3,5,3,6$ & $7,6,9,6,11$ & $16,12,20,4,20$ & $76,63,90,50,92$ \\
\hline $\begin{array}{l}\text { 4-Methyl-2- } \\
\text { methoxyphenol }\end{array}$ & n.d. & n.d. & n.d. & n.d. & n.d. & n.d. & $2,1,3,0.5,3$ & $11,6,14,6,21$ \\
\hline $\begin{array}{l}\text { 4-Ethyl-2- } \\
\text { methoxyphenol }\end{array}$ & n.d. & n.d. & n.d. & n.d. & n.d. & $0.2,0.2,0.2,0,0.2$ & $0.7,0.7,2,0.3,2$ & $8,3,12,3,18$ \\
\hline
\end{tabular}

\section{Electrophysiology with M. cuspidata}

Twenty-three compounds showed a response on the eight individuals tested in the GC-MS/EAD studies. Fourteen of these compounds were identified by means of their mass spectra and their standard retention time. Characteristic GC-MS/EAD chromatograms for each temperature are shown in Figure 1. 1-Pentanol, $\beta$-pinene, nonanal, hexanal, $\alpha$-pinene, 2-methoxyphenol, and heptanal are detected down to vapor pressures below $0.5 \mathrm{ppm}$ in the EAG experiments (Table 4). Furfural is detected below a vapor pressure of $2.9 \mathrm{ppm}$. 

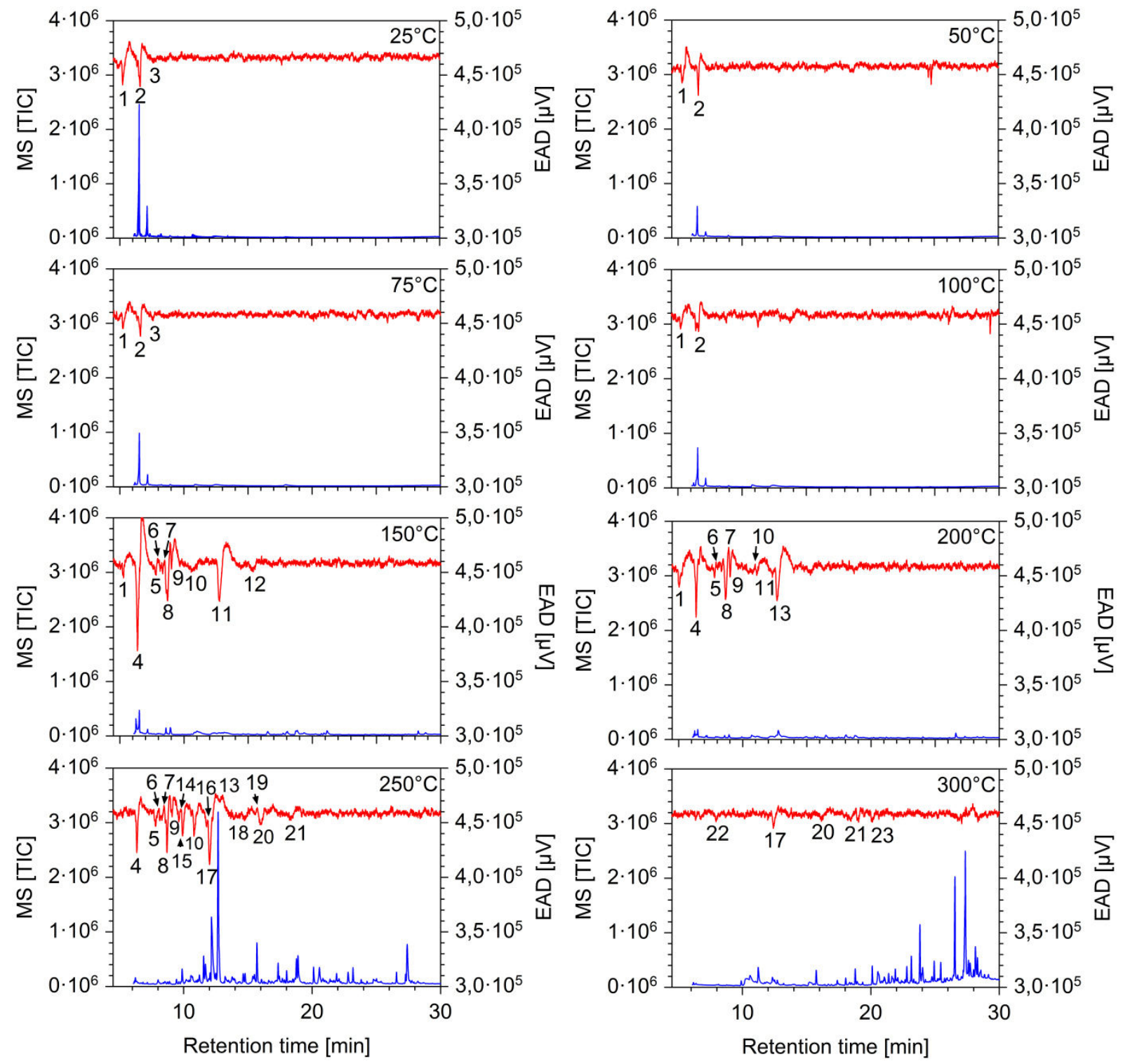

Fig. 1: GC-MS EAD results. The Figure shows representative GC-MS/EAD results for Melanophila cuspidata tested on samples of heated wood chips (Pinus sylvestris) in the temperature range of $25^{\circ} \mathrm{C}$ to $300^{\circ} \mathrm{C}$. 1: $\alpha$-pinene; $2: \beta$-pinene; 3 : 3-carene; 4: hexanal; 5: heptanal; 6: unidentified; 7: unidentified; 8: 1-pentanol; 9: 2-pentylfuran; 10: N,N-

dimethylformamide; 11: nonanal; 12: unidentified; 13: furfural; 14: unidentified; 15: hydroxyacetone; 16: unidentified; 17: acetic acid; 18: 5-methylfurfural; 19: unidentified; 20: 2-furanmethanol; 21: 2-methoxyphenol; 22: unidentified; 23: 4methyl-2-methoxyphenol.

\section{Behavioral studies with M. cuspidata}

The beetles were very active during the behavioral experiments and walked constantly from one side to the other. Therefore, most of the tested compounds showed a median in the range of $48 \%$ and $52 \%$ (Table 4). Most of the upper and lower quartiles showed values between $25 \%$ and $75 \%$. 1-Pentanol shows an upper quartile higher than $75 \%$ (upper quartile $=77 \%$ ) at a dilution of $10^{-2}(\mathrm{~g} / \mathrm{g})$. 3-Carene $(p=0.001)$ was significantly attractive at a dilution of $10^{-4}(\mathrm{~g} / \mathrm{g})$ and 2methoxyphenol $(p=0.022)$ and 5 -methylfurfural $(p=0.009)$ were significantly attractive at a dilution of $10^{-4}(\mathrm{~g} / \mathrm{g})$. All other compounds showed no significant attraction or repellence. The volatile mixture containing 2-methoxyphenol and 5-methylfurfural (1:1) was attractive at a dilution of $10^{-4}(\mathrm{~g} / \mathrm{g})$ $(p=0.032)$. 
Table 4: EAG and behavioral studies. The table shows the dose-response relation (EAG) and the behavioral response of Melanophila cuspidata towards compounds from heated wood flakes (Pinus sylvestris). A low vapor pressure of the $1^{\text {st }}$ significant dilution indicates a perception at low concentration in the EAG. The $p$-level of the behavioral responses was calculated by the $t$-Test or the $U$-test. Significant differences between the diluted compound and the paraffin oil control are indicated by circles or by the number of $\operatorname{stars}\left({ }^{\circ}: p<0.1,{ }^{*}: p<0.05,{ }^{* *}: p<0.01, * * *: p<0.001\right)$.

\begin{tabular}{|c|c|c|c|}
\hline Compound & $\begin{array}{c}\text { Vapor pressure } \\
\text { of } 1^{\text {st }} \text { significant } \\
\text { concentration } \\
\text { [ppm] }\end{array}$ & $\begin{array}{c}\text { P-level of } \\
\text { behavioral } \\
\text { response at } \\
\text { dilution of } \\
10^{-2}\end{array}$ & $\begin{array}{c}\text { P-level of } \\
\text { behavioral } \\
\text { response at } \\
\text { dilution of } 10^{-4}\end{array}$ \\
\hline 1-Pentanol & 0.003 & ${ }^{\circ} 0.054$ & 0.784 \\
\hline$\beta$-Pinene & 0.003 & 0.235 & 0.438 \\
\hline Nonanal & 0.007 & 0.584 & 0.693 \\
\hline Hexanal & 0.014 & 0.476 & ${ }^{\circ} 0.094$ \\
\hline$\alpha$-Pinene & 0.043 & 0.469 & 0.301 \\
\hline 2-Methoxyphenol & 0.234 & 0.281 & $* 0.022$ \\
\hline Heptanal & 0.500 & 0.885 & ${ }^{\circ} 0.096$ \\
\hline 2-Furanmethanol & 1.323 & ${ }^{\circ} 0.055$ & 0.992 \\
\hline 2-Pentylfurane & 2.626 & 0.520 & 0.978 \\
\hline Furfural & 2.899 & ${ }^{\circ} 0.088$ & 0.639 \\
\hline Hydroxyacetone & 3.835 & 0.887 & 0.419 \\
\hline Acetic acid & 5.499 & 0.595 & 0.533 \\
\hline 5-Methylfurfural & 8.372 & 0.898 & $* * 0.009$ \\
\hline 3-Carene & 48.36 & $* * * 0.001$ & 0.839 \\
\hline 4-Methyl-2-methoxyphenol & & 0.859 & 0.853 \\
\hline $\mathrm{N}, \mathrm{N}$-Dimethylformamide & & 0.522 & 0.171 \\
\hline Mixed group (5-Methylfurfural / 2-Methoxyphenol 1:1) & & & $* 0.032$ \\
\hline
\end{tabular}

Furfural $(p=0.088)$ and 2 -furanmethanol $(p=0.055)$ had a low but insignificant $p$ value.

\section{Sensor performance in the gas mixing equipment}

The nanoporous sensor responded towards furfural at a concentration of $\sim 14$ ppmv. Previous experiments already showed an increased performance of the nanoporous sensor in detecting the furfural peak (response of 1.05) in comparison to the performance of the bulk material, which showed a response of 0.1 (Eßinger et al., 2010). Therefore, the sensitive nanoporous sensor is used for the fire experiments in the burning chamber.

\section{Fire experiments in the burning chamber}

The temperature cycle allowed an extraction of selected sensor signals for a precise correlation to the volatile formation in the chamber. The quotient of the sensor signals $Y$ and $X$ is plotted against the feature $X$ (see inset Figure 2). That means that the relation of $X$ to $Y$ is correlated to the same $X . X$ and $Y$ are relative values expressing the conductance $(G)$ of the sensor. Monitoring $X$ and $Y$ allows to see the volatile induced change in conductance on the sensor surface. The development of the relation of the two features is shown in Figure 3. This relation does not allow a significant discrimination of the temperature steps between $25{ }^{\circ} \mathrm{C}$ and $150{ }^{\circ} \mathrm{C}(p>5 \%)$. The temperature stages of $200{ }^{\circ} \mathrm{C}$ and $250^{\circ} \mathrm{C}$ (heating stage) show a relation of the features that differs significantly from the fresh stage $(p<5 \%)$. All of the following temperature steps can be discriminated from each other 


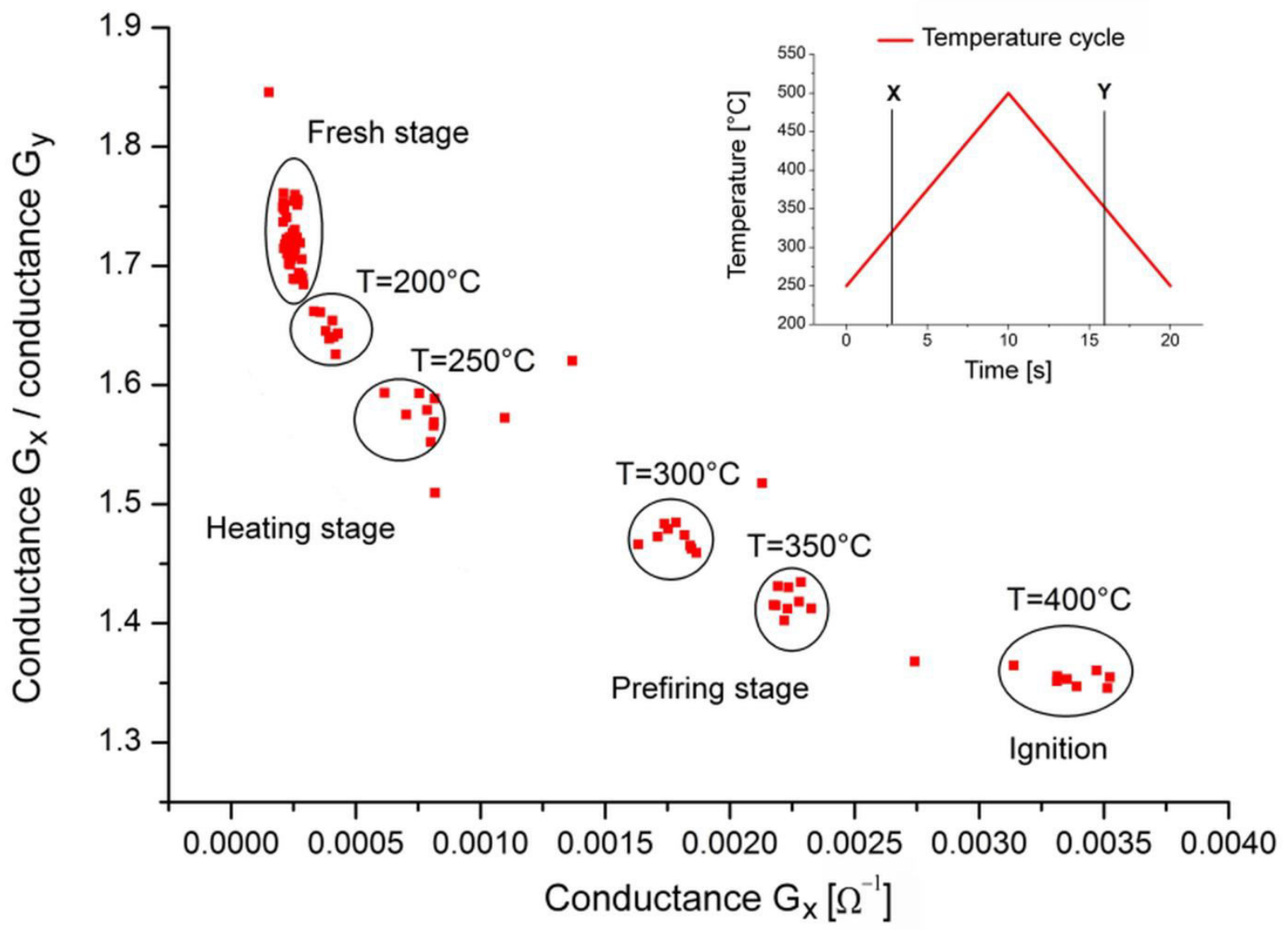

Fig. 2 shows the response (conductance, G) of a nanoporous WO3 sensor during the heating of wood strands in a burning chamber and the applied temperature cycle. $X$ and $Y$ (inset) are the points in the cycle, which were used for the discrimination between the fire phases.

significantly (heating stage-prefiring stage: $p<5 \%$; prefiring stage-ignition: $p<5 \%$ ). The wood chips began to smolder during the prefiring stage at $300{ }^{\circ} \mathrm{C}$ and $350{ }^{\circ} \mathrm{C}$. They began to burn in the ignition stage at $400{ }^{\circ} \mathrm{C}$. The door of the burning chamber was opened after the fire phase and the sensor signal returned to the fresh stage situation.

\section{MS analysis of furfural in the burning chamber}

The mass $\mathrm{m} / \mathrm{z}=96$ was detected at $300^{\circ} \mathrm{C}, 350^{\circ} \mathrm{C}$, and $400{ }^{\circ} \mathrm{C}$ (Figure 2). The abundance of ions with the mass/charge ratio of $\mathrm{m} / \mathrm{z}=96$ rose with increasing temperature in the prefiring stage and reached the highest level during inflammation at $400{ }^{\circ} \mathrm{C}$ (Figure 3).

\section{Discussion}

\section{Evaluation of a biomimetic early fire warning concept}

The monoterpenes $\alpha$-pinene and $\beta$-pinene are perceived by $M$. cuspidata at ppb-concentrations (Table 4) and are emitted at low concentrations (median < 20 ppmv in $30 \mathrm{~s}$ ) in the fresh stage and the heating stage of the wood chips (Table 2). $\alpha$-Pinene is reliably emitted with a minimum above 0 at all temperature steps of the fresh stage. The monoterpene 3-carene is perceived at higher concentrations and the beetles show highly significant attraction towards a $10^{-2}$ source in the walking assays. The compound is reliably emitted in the fresh stage, but not in the heating, prefiring or post- 


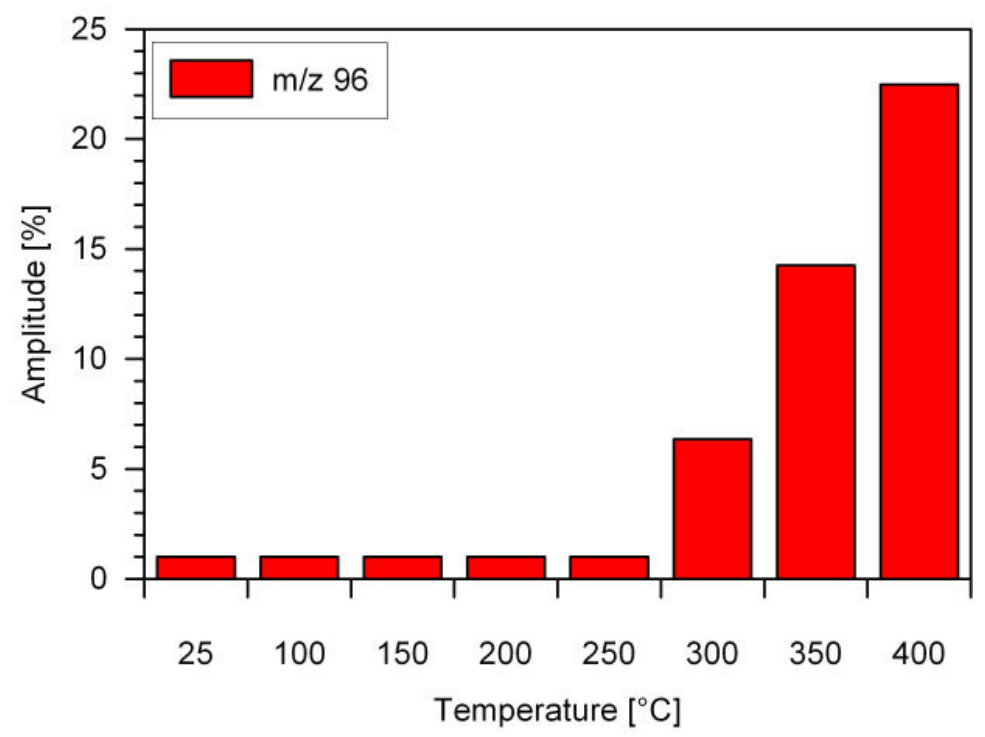

Fig. 3 shows the temperature dependent amplitude of the online mass-spectrometer detecting $\mathrm{m} / \mathrm{z}=96$ during the heating of wood strands in a burning chamber.

ignition stage (Table 2). It is typical for P. sylvestris (Granström 2002; Banerjee 2001; Englund et al., 2000 ), probably being a host plant volatile for $M$. cuspidata. Thus, $\alpha$-pinene and 3 -carene could allow the beetle to differentiate between the fresh stage and the stages of wood combustion.

1-Pentanol and hexanal are perceived at the low ppb range, but showed only slight and insignificant attraction in the walking bioassays. Both compounds are emitted from $150{ }^{\circ} \mathrm{C}$ to $250{ }^{\circ} \mathrm{C}$ at low concentrations (median < 20 ppmv / 30 s). This could explain the high sensitivity of the beetles towards these compounds. The occurrence of 1-pentanol and hexanal could inform the beetle about the beginning of the thermal oxidation of fatty acids (Risholm-Sudman et al., 1998; Svedberg et al., 2004; Arshadi, 2005; Roffael, 2006; Snyder et al., 1988; Manninen et al., 2002; Frankel, 1983). Especially hexanal is emitted reliably in all of the three stages. The beetles could differentiate between wood at elevated temperatures (later fresh stage, heating stage, or prefiring stage) and wood that is burned and not suitable for oviposition (post-ignition stage) by means of this compound.

5-Methylfurfural is perceived at the lower ppm-range and is also attractive for the beetles (Table 4). This compound is firstly discovered to play a role in the chemoecology of the genus Melanophila. 2Furanmethanol and furfural are perceived at the lower ppm-range, but showed only a slight and insignificant attraction in the walking bioassays. 5-Methylfurfural, 2-furanmethanol and furfural are products of hemicelluloses and cellulose degradation (Risholm-Sudman et al., 1998; Fengel and Wegener, 1984; Oasmaa et al., 2003; Tjeerdsma et al., 1998; Chheda et al., 2006) and 5methylfurfural and furfural were emitted during the thermal oxidation of cellulose (Table 3). A high furane emission might allow the beetle to differentiate between the post-ignition stage and the other stages of wood beneath charred bark. Especially furfural exhibits the highest reliable emission rate prior to ignition (Table 2 and 3 ).

2-Methoxyphenol is electrophysiologically active and behaviorally attractive down to the ppb-range. This confirms the results of former studies on M. acuminata, which is a fire beetle of the same genus (Schütz et al., 1999). 2-Methoxyphenol is a combustion product of lignin and emitted in high 
amounts after ignition (Azeez et al., 2010; Branca et al., 2006; Ingram et al., 2008; Karagoz et al., 2005). The results presented in Table 2 show low concentrations of 2 -methoxyphenol at $250^{\circ} \mathrm{C}$ and $300{ }^{\circ} \mathrm{C}$, because the ignitions took place between $250^{\circ} \mathrm{C}$ and $300^{\circ} \mathrm{C}$. The first combustion products of lignin in the wood flakes were collected shortly before ignition $\left(250{ }^{\circ} \mathrm{C}\right)$. The sampling at $300{ }^{\circ} \mathrm{C}$ collected only traces of the smoke, which were still present after the burning of the wood chips. The thermal oxidation of lignin resulted in higher emission rates of 2-methoxyphenol and proved its formation from lignin (Table 3). The presence of 2-methoxyphenol in the air around a charred stem can give no information about the physiological status of the wood beneath the bark. 2Methoxyphenol is most probably a far range volatile for the beetle and is used for a volatile based orientation towards wood land fires.

\section{Evaluation of the suitability of the biomimetic concept for the nanoporous $\mathrm{WO}_{3}$ sensor}

The chemoecological experiments with $M$. cuspidata resulted in a set of volatiles meeting the first criteria for marker volatiles. These volatiles are $\alpha$-pinene and 3 -carene for the differentiation between the fresh stage and the other stages, hexanal for the differentiation between the postignition stage and the other stages, the furans for the differentiation between the prefiring stage and the other stages, and 2-methoxyphenol for the detection of smoke during ignition. $\beta$-Pinene and 1pentanol are perceived by the beetle at low concentration but do not occur reliably in the trace analytical experiments (Table 2). It is possible that the compound can be detected by the highly sensitive antenna of the beetle (Table 4), but not by a GC-MS system. This shows the high sensitivity and selectivity of the biochemical detection in insects (Tegoni et al., 2004), which can perceive even single molecules with their antenna (Pelosi and Maida 1995). A transfer from the olfaction of insects to technical sensors is restricted by the lower performances of the technical sensors.

The results of the GC-MS/SOMSA experiments with the nanoporous sensor suggested a high sensitivity towards furfural. A concentration of $\sim 140 \mathrm{ppmv} / \mathrm{s}$ led to a sensor response of $525 \mathrm{mV}$. The sensitivity of the nanoporous sensor is confirmed by the results of the burning chamber experiment, where furfural was detected in the prefiring stage. A strong increase of the furfural emission between the heating stage and the prefiring stage (Figure 2) led to a strong change in the sensor signal (Figure 3). The nanostructured sensor exhibits a good performance in early fire detection in this experiment. However, the sensor signal already changed significantly after the fresh stage, although the portable mass spectrometer did not detect the mass $\mathrm{m} / \mathrm{z}=96$ in the heated stage. The nanoporous sensor corresponded to other volatiles than furfural. This shows that the nanoporous sensor allowed a sensitive detection of furfural, but could not completely eliminate influences by other gases. Field studies have to be performed in order to evaluate the sensitivity and selectivity of the proposed nanoporous sensor under complex conditions. Previous investigations on nanoporous $\mathrm{WO}_{3}$ sensors showed that the temperature variation technique is capable of discriminating between furfural (as a fire indicator) and alpha-pinene (as a ubiquitous emission of heated as well as of non-heated wood) (Paczkowski et al., 2011). This aspect will be important in field tests, because an overlapping of terpene and furfural emissions can be expected in field tests.

The third requirement for marker volatiles was the sole emission by wood and not by other sources. Although furfural is reported to form during cooking of meat (Meynier et al., 2000), this cannot be regarded as a source leading to false alarms. The compound does not form during the decomposition 
of vertebrates (Paczkowski and Schütz 2011). Therefore, a decaying mouse cadaver in a wood storage would not irritate a sensor system, while it is possible that furfural emits from heated carcasses during wood fires. Some species of cellulose degrading fungi might emit low concentrations of furfural (Sunesson et al., 1996), but this compound is not frequently reported in literature on wood and tree fungi (Thakeow et al., 2008). Furfural is known as a possible product of biochemical reactions in soil and litter (Leff and Fierer 2008), which could irritate a sensor used for wood storages on unsealed outdoor areas. This has to be considered for field studies on the proposed nanoporous sensor.

\section{Conclusions}

The volatile emission during the thermal oxidation of wood chips or wood strands is correlated to the temperature stage of the chips. The investigation of the chemoecology of $M$. cuspidata revealed several marker volatile candidates for the differentiation between the proposed stages of thermal oxidation. This indicates that $M$. cuspidata identifies appropriate oviposition places by means of olfaction and that its selective olfaction can be used for early fire warning systems. Furfural is the most promising compound to meet the three criteria for marker volatiles, because it is emitted reliably at high rates prior to ignition. The olfaction of $M$. cuspidata suggests to include $\alpha$-pinene, hexanal, and 2-methoxyphenol in the sensor concept, but this will require highly sensitive and selective gas sensors (Paczkowski et al., 2012). A self prepared semiconductor metal oxide sensor tuned to the selective detection of furfural was able to differentiate between the fresh stage, the heated stage, the prefiring stage, and the ignition stage of wood strands in a burning chamber. This sensor has to be integrated into multisensor-systems and promises a large application range in the wood processing industry or in wood storages. It was successfully tested as a part of a multi gas sensor array in the atmosphere of a kiln drier in a facility of the Glunz AG (Nettgau, Germany).

\section{Acknowledgement}

We like to thank Prof. Dr. Helmut Schmitz for the supply with M. cuspidata. We like to thank Dr. Bernhard Weissbecker and Dr. Gerrit Holighaus for their helpful comments on our methods and results. We like to thank Dr. Puttfarken and Alfred Engelshove from the Glunz AG for supporting our studies and Arnim Titze and Heino Schmeiß from the Glunz AG for their technical support during our sensor field tests. The olfactometer was kindly provided by the DFG Priority Programme/ SPP 1392: Integrative Analysis of Olfaction. The work of Marta Paczkowska was kindly supported by the BMBF (Bundesministerium für Bildung und Forschung). The work of Sebastian Paczkowski was kindly supported by the Cusanuswerk, Bischöfliche Studienförderung.

\section{References}

Arshadi,M. (2005) Emission of volatile organic compounds from softwood pellets during storage. Forest Products Journal, 55, 132-135.

Azeez,A.M., Meier,D., Odermatt,J. and Willner,T. (2010) Fast pyrolysis of African and European lignocellulosic biomasses using Py-GC/MS and fluidized bed reactor. Energy \& Fuels, 24, 2078-2085. 
Banerjee,S. (2001) Mechanisms of terpene release during sawdust and flake drying. Holzforschung, $55,413-416$.

Branca,C., Di Blasi,C. and Elefante,R. (2006) Devolatilization of conventional pyrolysis oils generated from biomass and cellulose. Energy \& Fuels, 20, 2253-2261.

Castello,G., Moretti,P. and Vezzani,S. (2009) Retention models for programmed gas chromatography. Journal of Chromatography A. 1216, 1607-1623.

Chheda,J.N., Roman-Leshkov,Y. and Dumesic,J.A. (2006) Production of 5-hydroxymethylfurfural and furfural by dehydration of biomass-derived mono- and poly-saccharides. Green Chemistry, 9, 342350.

Englund,F. and Nussbaum,R.M. (2000) Monoterpenes in scots pine and norway spruce and their emission during kiln drying. Holzforschung, 54, 449-456.

Eßinger, T. M., Becker, B., Sauerwald, T., Kohl, C.-D., Paczkowski, S. and Schütz, S. (2010) Volatile gas sensors for large-scale drying of wood. Sensoren und Messsysteme - 15. ITG/GMA-Fachtagung. pp. 1-4, Nürnberg, Germany.

Evans,W.G. (2010) Reproductive role of infrared radiation sensors of Melanophila acuminata (coleoptera: buprestidae) at forest fires. Annals of the Entomological Society of America. 103, 823826.

Fengel,D. and Wegener,G. (1984) Wood: Chemistry, ultrastructure, reactions. De Gruyter Inc., Boston, USA.

Frankel,E.N. (1983) Volatile lipid oxidation-products. Progress in Lipid Research, 22, 1-33.

Gramm,A. and Schütze,A. (2003) High performance solvent vapor identification with a two sensor array using temperature cycling and pattern classification. Sensors and Actuators B-Chemical, 95, 5865.

Gutmacher,D., Hoefer,U. and Wöllenstein,J. (2011) Gas sensor technologies for fire detection. Sensors and Actuators B-Chemical. 175, 40-45.

Granström,K. (2002) Emissions of monoterpenes and VOCs during drying of sawdust in a spouted bed. Forest Products Journal, 53, 48-55.

Hofmann,T., Schieberle,P., Krummel,C., Freiling,A., Bock,J., Heinert,L. and Kohl, C.-D. (1997) High resolution gas chromatography/selective odorant measurement by multisensor array (HRGC/SOMSA): a useful approach to standardise multisensor arrays for use in the detection of key food odorants. Sensors and Actuators: B-Chemical. 41, 81-87.

Ingram,L., Mohan,D., Bricka,M., Steele,P., Strobel,D., Crocker,D., Mitchell,B., Mohammad,J., Cantrell,K. and Pittman,C.U. (2008) Pyrolysis of wood and bark in an auger reactor: Physical properties and chemical analysis of the produced bio-oils. Energy \& Fuels, 22, 614-625.

Kakati,N., Jee,S.H., Kim,S.H., Lee,H.K. and Yoon,Y.S. (2009) Sensitive enhancement of ZnO nanorod gas sensors with surface modification by an InSb thin film. Japaneese Journal of Applied Physics. 48, 105002. 
Karagoz,S., Bhaskar,T., Muto,A. and Sakata,Y. (2005) Comparative studies of oil compositions produced from sawdust, rice husk, lignin and cellulose by hydrothermal treatment. Fuel, 84, 875-884.

Kollmann,F. (1982) Technologie des Holzes und der Holzwerkstoffe: Anatomie und Pathologie, Chemie, Physik, Elastizität und Festigkeit. Springer, Berlin, Germany.

Kreiss,E., Schmitz,H. and Gebhardt,M. (2007) Electrophysiological characterisation of the infrared organ of the Australian "Little Ash Beetle" Acanthocnemus nigricans (Coleoptera, Acanthocnemidae). Journal of Comparative Physiology A-Neuroethology Sensory Neural and Behavioral Physiology, 193, 729-739.

Leff,J.W. and Fierer,N. (2008) Volatile organic compound (VOC) emissions from soil and litter samples. Soil Biology and Biochemistry. 40, 1629-1636.

Manninen,A.-M., Pasanen,P. and Holopainen,J.K. (2002) Comparing the VOC emissions between airdried and heat-treated scots pine wood. Atmospheric Environment. 36, 1763-1768.

Martel,V. and Boivin,G. (2011) Do choice tests really test choice. Journal of Insect Behavior, 24, 329336.

Meynier,A. and Mottram, D.S. (2000) The effect of $\mathrm{pH}$ on the formation of volatile compounds in meat-related model systems. Food Chemistry 52, 361-366.

Oasmaa,A., Kuoppala,E. and Solantausta,Y. (2003) Fast pyrolysis of forestry residue. 2. Physicochemical composition of product liquid. Energy \& Fuels, 17, 433-443.

Paczkowski,S. and Schütz,S. (2011) Post-mortem volatiles of vertebrate tissue. Applied Microbiology and Biotechnology, 91, 917-935.

Paczkowski,S., Sauerwald,T., Weiß.A., Bauer,M., Kohl,C.-D., and Schütz,S. (2011) Biomimetic gas sensors for large-scale drying of wood particles. Martin-Palma, R. J. and Lakhtakia, A. 7975, 797505797505-8. Proceedings of the SPIE: Bioinspiration, Biomimetics, and Bioreplication, San Diego, USA.

Pelosi,P. and Maida,R. (1995) Odorant-binding proteins in insects. Comparative Biochemistry and Physiology B-Biochemistry \& Molecular Biology, 111, 503-514.

Piskorz,J., Radlein,D. and Scott,D.S. (1986) On the mechanism of the rapid pyrolysis of cellulose. Journal of Analytical and Applied Pyrolysis, 9, 121-137.

Risholm-Sudman,M., Lundgren,M., Vestin,E. and Herder,P. (1998) Emissions of acetic acid and other volatile organic compounds from different species of solid wood. Holz als Roh- und Werkstoff, 56, 125-129.

Roffael,E. (2006) Volatile organic compounds and formaldehyde in nature, wood and wood based panels. Holz als Roh- und Werkstoff, 64, 144-149.

Rossinyol,E. and Tian,B. (2005) Nanostructured metal oxides synthesized by hard template method for gas sensing applications. Sensors and Actuators B. 109, 57-63.

Schmitz,H., Bleckmann,H. and Mürtz,M. (1997) Infrared detection in a beetle. Nature. 386, 773-774. 
Schütz,S., Weissbecker,B., Hummel,H.E., Apel,K.H., Schmitz,H. and Bleckmann,H. (1999) Insect antenna as a smoke detector. Nature, 398, 298-299.

Semancik,S., Cavicchi,R.E., Wheeler,M.C., Tiffany,J.E., Poirier,G.E., Walton,R.M., Suehle,J.S., Panchapakesan,B. and DeVoe,D.L. (2001) Microhotplate platforms for chemical sensor research. Sensors and Actuators: B-Chemical. 77, 579-591.

Simoneit,H., Maunu,S.L., Sundholm,F., Jämsä,S. and Viitaniemi,P. (2002) Magnetic resonance studies of thermally modified wood. Holzforschung. 56, 648-654.

Snyder JM, Frankel EN, Selke E and Warner K. (1988) Comparison of gas chromatographic methods for volatile lipid oxidation compounds in soybean oil. Journal of the American Oil Chemistry Society. 65, 1617-1620.

Sunesson,A.L., Nilsson,C.A., Andersson,B. and Blomquist,G. (1996) Volatile metabolites produced by two fungal species cultivated on building materials. Annals of Occupational Hygiene, 40, 397-410.

Svedberg,U.R.A., Högberg,H.E., Högberg,J. and Galle,B. (2004) Emission of hexanal and carbon monoxide from storage of wood pellets, a potential occupational and domestic health hazard. Annals of Occupational Hygiene, 48, 339-349.

Tegoni,M., Campanacci,V. and Cambillau,C. (2004) Structural aspects of sexual attraction and chemical communlication in insects. Trends in Biochemical Sciences, 29, 257-264.

Thakeow,P., Angeli,S., Weissbecker,B. and Schütz,S. (2008) Antennal and behavioral responses of Cis boleti to fungal odor of Trametes gibbosa. Chemical Senses, 33, 379-387.

Thakeow,P., Angeli,S., Weissbecker,B. and Schütz,S. (2008) Antennal and behavioral responses of Cis boleti to fungal odor of Trametes gibbosa. Chemical Senses, 33, 379-387.

Weissbecker,B., Holighaus,G. and Schütz,S. (2004) Gas chromatography with mass spectrometric and electroantennographic detection: analysis of wood odorants by direct coupling of insect olfaction and mass spectrometry. Journal of Chromatography A, 1056, 209-216.

Zhang,B., Huang,H.J. and Ramaswamy,S. (2008) Reaction kinetics of the hydrothermal treatment of lignin. Applied Biochemistry and Biotechnology, 147, 119-131. 


\section{The olfaction of the fire beetle Merimna atrata as a model for early fire detection in eucalypt forests}

Corresponding author: Sebastian Paczkowski ${ }^{\mathrm{a} 1}$, Dept. of Forest Zoology and Forest Conservation, Büsgen-Institute, Georg-August-University, Göttingen, Germany. email: SebastianPaczkowski@gmx.de, Tel.: +49-07666-6026417, fax: fax: +49-551-39-22089

Marta Paczkowskaa, Dept. of Forest Zoology and Forest Conservation, Büsgen-Institute, GeorgAugust-University, Göttingen, Germany. email: mpaczko@gwdg.de

Sara Nickea , Dept. of Forest Zoology and Forest Conservation, Büsgen-Institute, Georg-AugustUniversity, Göttingen, Germany, e-Mail: snicke@gwdg.de

Prof. Dr. Stefan Schütz ${ }^{a}$, Dept. of Forest Zoology and Forest Conservation, Büsgen-Institute, GeorgAugust-University, Göttingen, Germany, email: stefan.schuetz@forst.uni-goettingen.de

\subsection{Abstract}

Volatile based early fire warning systems for wood land fires could shorten the time between the outbreak of a fire and the arrival of fire crews. This can prevent the development of crown fires, which are harder to control than ground fires. In this study, the temperature dependent volatile emission of tree biomass (Eucalyptus globulus wood and leaves) were analyzed by GC-MS and the 20 identified compounds were quantified and tested on the antennae of the fire beetle Merimna atrata (EAG). $\alpha$-Pinene, eucalyptol, furfural, and 2-furanmethanol are reliably emitted in distinct stages of heated wood, show the highest emission rates and are electrophysiological active on the antenna of the beetle. The role of these compounds for early fire warning systems in eucalypt forests is discussed.

Additional Keywords: Early fire detection; fire management; volatiles; gas sensor

\subsection{Introduction}

Wood land fires are important for the Western Australian ecosystem, but especially fast spreading crown fires can be a serious threat for the people living in this region. Therefore, fire management plans, including prescribed burnings (Wilson et al., 2010), minimize the risk of fast spreading fires. The early detection of emerging fires is an important part in fire management. While ground fires can be controlled by fire crews, crown fires can spread fast and are much harder to control. Therefore, the time between the ignition of a fire and the arrival of fire crews is important for the prevention of large fires. In order to minimize that time, gas sensors can be used for the detection of smoke and

\footnotetext{
${ }^{1}$ Permanent address: Schwarzwaldstr. 48, 79211 Denzlingen, Germany
} 
wood combustion products emitted even prior to ignition (Browne, 1958; Greenberg et al., 2006; Eßinger et al., 2010; Paczkowski et al., 2011).

These combustion products can be used to assess increasing fire risk or even to predict fires before they ignite. As combusting wood or leaves yield many different volatiles, reliable marker compounds have to be selected, which fulfill the three following criteria. Firstly, a marker volatile should be reliably emitted at a certain stage prior to ignition. Secondly, the selected combustion volatiles should not be emitted by other sources than combusting wood or leaves in order to reduce false alarms. These two criteria are fulfilled by compounds detected by $M$. atrata, because during millions of years of evolution the beetles' olfaction has been adapted to volatiles reliably emitted by heated trees. Thirdly, an emission of large quantities of the selected volatiles during combustion will ensure their detectebility by gas sensor systems (Kohl, 2003). Although gas sensors can detect isolated volatiles at very low concentration, the same volatile might not be detectable in a volatile mixture, since gas sensors are not very selective.

Because of the high diversity of tree combustion volatiles it would be very time consuming to identify volatiles that fulfill all of the three criteria. In order to enable a fast selection, the olfaction of the fire beetle $M$. atrata is utilized for the selection of marker volatiles. This beetle is reported to be attracted by Australian wood land fires in large numbers. It selects stems for mating and oviposition on the freshly burned area, which is a typical behavior for fire associated insects (Schütz et al., 1999; Paczkowski et al., 2011, Evans, 2010; Schmitz et al., 1997; Kreiss et al., 2007). The beetles select stems with heated but unburned wood tissue. Such stems provide nutrients for the larval development, but are damaged and cannot defend themselves against the feeding of the larvae. The beetles have to distinguish between intact, heated, and burned stems in order to find the appropriate oviposition place. The optical impression of the stems cannot be used for an assessment of the physiological status, because all stems are charred after a fire. However, this information could be gained by volatile compounds that form while wood or leaves are heated and it is highly probable that pyrophilic insects are adapted to sense marker volatiles of this heating process. In this study, the olfaction of M.atrata is used to select marker volatiles for early fire warning gas sensor systems.

\subsection{Material and Methods}

\subsubsection{Thermal oxidation experiments with E. globulus tissues}

Thermal oxidation experiments were performed with $2 \mathrm{~g}$ wood chips, $2 \mathrm{~g}$ leaves, and $1 \mathrm{~g}$ wood and $1 \mathrm{~g}$ leaves of Eucalyptus globulus. In order to get five replications for each type, five $E$. globulus branches with leaves were collected from trees in different botanical gardens in Germany (Bremen, Marburg, Leipzig, Halle, Bochum). The preparation of the chips and the heating of the chips and the leaves was done according to the method described in Paczkowski et al., 2013 (pp.100-101).

\subsubsection{Trace analysis with the volatile samples from E. globulus tissues}

The trace analysis was performed according to the method described in Paczkowski et al., 2012 (pp.71). 


\subsubsection{Quantification of volatiles}

The volatiles were quantified according to a method of Paczkowski et al., 2013 (pp.101).

\subsubsection{Insect collection and rearing}

Adult Merimna atrata were collected in eucalypt forests shortly after wildfires in the Dwellingup State Forest, Western Australia, and in the Mundaring State Forest, Western Australia, in summer 2009 and 2010 and in eucalypt forest areas at Jurien Bay, Western Australia, in summer 2010 and 2011. The beetles were found on charred stems or brushes one to ten days after a fire. They were kept in plastic containers with a $10 \%$ saccharose sugar water and water diet. After the transport to Germany, they were kept at $25 \pm 1^{\circ} \mathrm{C}, 40 \%$ relative humidity and a $12 / 12$ day/night period.

\subsubsection{EAG with M. atrata}

The electroantennogramm recordings were performed according to a method described in Paczkowski et al., 2012 (p.72).

\subsection{Results}

\subsubsection{Trace analysis with the samples from E. globulus tissues}

20 compounds of the chemical groups of terpenes, furans, methoxyphenols, and benzenes were detected in the trace analytical experiments (Table 1). The group of terpenes comprises nine compounds (48\%), the group of furans comprises five compounds (24\%), the group of methoxyphenols comprises five compounds (24\%), and the group of benzenes one compound (4\%).

Heated wood yields only few terpenes (Table 2). Furans and methoxyphenols are emitted because of hemicelluloses, cellulose, and lignin combustion in the temperature range from $200^{\circ} \mathrm{C}$ to $300^{\circ} \mathrm{C}$ (Azeez et al., 2010; Branca et al., 2006; Chedda et al., 2006; Ingram et al., 2008; Karagoz et al., 2005; Malekina et al., 2009; Oasmaa et al., 2003; Paczkowski et al., 2010). Furfural is emitted at the highest concentration of all compounds quantified with a maximum of $514 \mathrm{ng} / \mathrm{L}$ at $250^{\circ} \mathrm{C}$. 2-Furanmethanol is emitted at high emission rates $(8-100 \mathrm{ng} / \mathrm{L})$ from $200^{\circ} \mathrm{C}$ to $300^{\circ} \mathrm{C}$. The methoxyphenols 2methoxyphenol and 4-methyl-2-methoxyphenol increase in concentration from $250^{\circ} \mathrm{C}$ to $300^{\circ} \mathrm{C}$ during the ignition of the wood tissue.

Heated leaves yield most of the identified terpenes. Eucalyptol, $\alpha$-pinene, $\beta$-pinene, limonene, and aromadendrene are already emitted at room temperature, while camphene, $\beta$-myrcene, camphor and epiglobulol are emitted at elevated temperatures (Chalchat et al., 2009; Malekina et al., 2009; Paczkowski et al., 2011). Eucalyptol is the only compound that is emitted throughout the heating process in the wood and leave samples and in the leave samples with an emission maximum at $200^{\circ} \mathrm{C}$. Between $200^{\circ} \mathrm{C}$ and $300^{\circ} \mathrm{C}$, 1-isopropyl-4-methylbenzene is emitted in increasing concentration. 5-Methylfurfural, 5-hydroxymethylfurfural, 2-methoxyphenol, 4-methyl-2methoxyphenol, 4-ethyl-2-methoxyphenol, and 2-methoxy-4-formylphenol are emitted at low concentrations up to $300^{\circ} \mathrm{C}$.

The volatile emissions of heated wood and leave comprise all of the listed volatiles besides the terpene camphor. The wood and leaf samples and the leaf samples emitted comparable quantities of terpenes. 
Table 1: Identified volatiles from E. globulus wood, leaves, and wood and leaves. The Table shows the identified volatile combustion products of $E$. globulus leaves, wood or wood and leaves in the order of their retention index on a nonpolar column. A high vapor pressure indicates a high volatility.

Compound

TDS (LRI)

HP-5MS

Terpenes

$\alpha$-Pinene

Camphene

$\beta$-Pinene

$\beta$-Myrcene

Limonene

Eucalyptol

Camphor

Aromadendrene

Epiglobulol

Furanes

Furfural

2-Furanmethanol

5-Methyl-2(5)-Furanone

5-Methylfurfural

5-Hydroxymethylfurfural

Methoxyphenols

2-Methoxyphenol

4-Methyl-2-methoxypheno

4-Ethyl-2-methoxyphenol

2-Methoxy-4-(2)-propenylphenol

2-Methoxy-4-formylphenol

Benzenes

1-Isopropyl-4-methylbenzene
Vapor

Authentic standards (company, purity, CAS No.)

pressure

(SATP)

[hPa]

Merck, 98\%, 80-56-4 3.49

SAFC, 95\%, 79-92-5 3.38

Fluka, 98\%, 127-91-3 2.4

Fluka, 95\%, 123-35-3 2.29

Merck, 98\%, 138-86-3 1.54

Aldrich, 98\%, 470-82-6 $\quad 1.9$

SAFC, 97\%, 21368-68-3 4

Merck, 98\%, 489-39-4 $\quad 0.0226$

SAFC, 98\%, 88728-58-9 0.03

Acros, 99\%, 98-01-1 2.234

Merck, 98\%, 98-00-0 1.009

Merck, 98\%, 591-11-7 0.197

Acros, 98\%, 620-02-0 $\quad 0.644$

Aldrich, 95\%, 67-47-0 $\quad 0.001$

Fluka, 98\%, 90-05-1 $\quad 0.179$

Aldrich, 98\%, 93-51-6 $\quad 0.0783$

SAFC, 98\%, 2785-89-9 $\quad 0.0173$

Merck, 98\%, 97-53-0 $\quad 0.0104$

Merck, 99\%, 121-33-5 0.00194

Aldrich, 98\%, 99-87-6 $\quad 1.46$ 
Table 2: Quantification of volatiles from E. globulus wood, leaves, and wood and leaves. The table shows the emission rates of the volatile compounds quantified in the samples of $E$. globulus

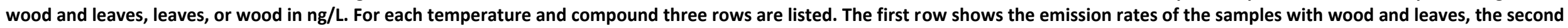
row shows the emission rates of the samples with wood and the third row shows the emission rates of the samples with leaves. The order of the numbers is: median, minimum, maximum, lower quartile, upper quartile. The median displays the average concentration in all samples per stage. If no value is given for the minimum the compound was absent in at least one sample. Results on the thermal oxidation of cellulose and lignin are presented in Table 3, p.106 of this thesis.

\begin{tabular}{|c|c|c|c|c|c|c|c|c|}
\hline \multirow{2}{*}{ Compound } & $25^{\circ} \mathrm{C}$ & $50^{\circ} \mathrm{C}$ & $75^{\circ} \mathrm{C}$ & $100^{\circ} \mathrm{C}$ & $150^{\circ} \mathrm{C}$ & $200^{\circ} \mathrm{C}$ & $250^{\circ} \mathrm{C}$ & $300^{\circ} \mathrm{C}$ \\
\hline & \multicolumn{5}{|c|}{ Fresh stage } & Heated stage & Prefiring stage & Post-Ignition \\
\hline \multicolumn{9}{|l|}{ Terpenes } \\
\hline$\alpha$-Pinene & $\begin{array}{l}9,1,41,2,26 \\
7,0,33,1,2 \\
12,2,33,9,30\end{array}$ & $\begin{array}{l}4,1,19,1,5 \\
4,0,20,0,1 \\
18,9,30,12,27\end{array}$ & $\begin{array}{l}12,2,19,1,5 \\
2,0,7,0,1 \\
12,4,137,12,17\end{array}$ & $\begin{array}{l}8,4,154,6,10 \\
2,0,7,0,1 \\
29,22,211,28,62\end{array}$ & $\begin{array}{l}70,9,256,59,188 \\
2,0,7,0,2 \\
94,75,324,83,101\end{array}$ & $\begin{array}{l}62,7,91,34,70 \\
4,0,18,0,0 \\
107,55,220,101,134\end{array}$ & $\begin{array}{l}14,0,32,8,20 \\
0,0,0,0,0 \\
30,19,107,28,44\end{array}$ & $\begin{array}{l}0,0,0,0,0 \\
0,0,0,0,0 \\
0,0,0,0,0\end{array}$ \\
\hline Camphene & $\begin{array}{l}0,0,0,0,0 \\
0,0,0,0,0 \\
0,0,0,0,0 \\
\end{array}$ & $\begin{array}{l}0,0,0,0,0 \\
0,0,0,0,0 \\
0,0,0,0,0 \\
\end{array}$ & $\begin{array}{l}0,0,0,0,0 \\
0,0,0,0,0 \\
0,0,0,0,0 \\
\end{array}$ & $\begin{array}{l}0,0,0,0,0 \\
0,0,0,0,0 \\
0,0,0,0,0 \\
\end{array}$ & $\begin{array}{l}0,0,0,0,0 \\
0,0,0,0,0 \\
0,0,0,0,0 \\
\end{array}$ & $\begin{array}{l}1,0,3,0,2 \\
0,0,0,0,0 \\
4,0,7,3,5 \\
\end{array}$ & $\begin{array}{l}3,1,8,2,3 \\
0,0,0,0,0 \\
11,3,22,8,20 \\
\end{array}$ & $\begin{array}{l}0,0,0,0,0 \\
0,0,0,0,0 \\
13,0,24,7,16 \\
\end{array}$ \\
\hline$\beta$-Pinene & $\begin{array}{l}0,0,1,0,1 \\
0,0,1,0,0 \\
0,0,1,0,1 \\
\end{array}$ & $\begin{array}{l}0,0,1,0,0 \\
0,0,0,0,0 \\
0,0,1,0,1 \\
\end{array}$ & $\begin{array}{l}0,0,5,0,1 \\
0,0,0,0,0 \\
0,0,3,0,0 \\
\end{array}$ & $\begin{array}{l}1,0,4,0,1 \\
0,0,0,0,0 \\
0,0,6,0,1 \\
\end{array}$ & $\begin{array}{l}1,1,4,1,2 \\
0,0,0,0,0 \\
1,0,4,1,1 \\
\end{array}$ & $\begin{array}{l}2,1,3,1,2 \\
0,0,1,0,0 \\
2,2,6,2,3 \\
\end{array}$ & $\begin{array}{l}0,0,2,0,0 \\
0,0,0,0,0 \\
1,0,2,0,2 \\
\end{array}$ & $\begin{array}{l}0,0,0,0,0 \\
0,0,0,0,0 \\
0,0,0,0,0 \\
\end{array}$ \\
\hline$\beta$-Myrcene & $\begin{array}{l}0,0,2,0,0 \\
0,0,0,0,0 \\
0,0,0,0,0\end{array}$ & $\begin{array}{l}0,0,1,0,0 \\
0,0,0,0,0 \\
0,0,0,0,0\end{array}$ & $\begin{array}{l}0,0,3,0,0 \\
0,0,0,0,0 \\
0,0,1,0,0\end{array}$ & $\begin{array}{l}0,0,6,0,1 \\
0,0,0,0,0 \\
0,0,2,0,1\end{array}$ & $\begin{array}{l}0,0,3,0,1 \\
0,0,0,0,0 \\
0,0,2,0,1\end{array}$ & $\begin{array}{l}0,0,0,0,0 \\
0,0,0,0,0 \\
0,0,0,0,0\end{array}$ & $\begin{array}{l}0,0,0,0,0 \\
0,0,0,0,0 \\
0,0,0,0,0 \\
\end{array}$ & $\begin{array}{l}0,0,0,0,0 \\
0,0,0,0,0 \\
0,0,0,0,0 \\
\end{array}$ \\
\hline Limonene & $\begin{array}{l}0,0,13,0,0 \\
0,0,0,0,0 \\
1,0,10,0,1 \\
\end{array}$ & $\begin{array}{l}1,0,6,0,1 \\
0,0,1,0,0 \\
1,0,10,1,1 \\
\end{array}$ & $\begin{array}{l}1,0,26,0,2 \\
0,0,0,0,0 \\
0,0,39,0,1 \\
\end{array}$ & $\begin{array}{l}1,0,12,0,2 \\
0,0,0,0,0 \\
1,0,65,0,3 \\
\end{array}$ & $\begin{array}{l}9,0,55,3,10 \\
0,0,0,0,0 \\
4,0,54,0,9 \\
\end{array}$ & \begin{tabular}{l|}
$10,3,46,4,40$ \\
$1,0,4,0,0$ \\
$27,0,37,18,31$ \\
\end{tabular} & $\begin{array}{l}44,17,46,19,45 \\
0,0,0,0,0 \\
55,13,127,34,107 \\
\end{array}$ & $\begin{array}{l}0,0,0,0,0 \\
0,0,0,0,0 \\
44,10,141,32,55 \\
\end{array}$ \\
\hline Eucalyptol & $\begin{array}{l}38,6,163,30,57 \\
21,0,98,0,5 \\
23,7,107,13,66 \\
\end{array}$ & $\begin{array}{l}31,8,80,30,57 \\
3,0,12,0,1 \\
80,12,107,17,83 \\
\end{array}$ & $\begin{array}{l}71,36,400,53,148 \\
1,0,5,0,1 \\
53,11,257,31,112 \\
\end{array}$ & $\begin{array}{l}81,55,387,64,82 \\
1,0,5,0,1 \\
57,34,316,44,115 \\
\end{array}$ & $\begin{array}{l}297,74,652,209,471 \\
5,0,22,0,1 \\
124,44,518,77,291 \\
\end{array}$ & $\begin{array}{l}323,162,294,200,287 \\
1,0,3,0,1 \\
250,85,500,132,270\end{array}$ & $\begin{array}{l}232,162,294,201,287 \\
1,0,2,0,1 \\
150,73,352,115,234\end{array}$ & $\begin{array}{l}0,0,9,0,5 \\
0,0,0,0,0 \\
87,3,351,61,93 \\
\end{array}$ \\
\hline Camphor & $\begin{array}{l}0,0,0,0,0 \\
0,0,0,0,0 \\
0,0,0,0,0 \\
\end{array}$ & $\begin{array}{l}0,0,0,0,0 \\
0,0,0,0,0 \\
0,0,0,0,0 \\
\end{array}$ & $\begin{array}{l}0,0,0,0,0 \\
0,0,0,0,0 \\
0,0,0,0,0\end{array}$ & $\begin{array}{l}0,0,0,0,0 \\
0,0,0,0,0 \\
0,0,0,0,0\end{array}$ & $\begin{array}{l}0,0,0,0,0 \\
0,0,0,0,0 \\
0,0,0,0,0\end{array}$ & $\begin{array}{l}0,0,0,0,0 \\
0,0,0,0,0 \\
0,0,0,0,0 \\
\end{array}$ & $\begin{array}{l}0,0,0,0,0 \\
0,0,0,0,0 \\
0,0,0,0,0 \\
\end{array}$ & $\begin{array}{l}0,0,0,0,0 \\
0,0,0,0,0 \\
1,0,1,0,1 \\
\end{array}$ \\
\hline Aromadendrene & $\begin{array}{l}0,0,4,0,2 \\
3,0,14,0,0 \\
3,0,8,0,3 \\
\end{array}$ & $\begin{array}{l}2,0,11,1,4 \\
3,0,15,0,0 \\
5,1,10,1,7 \\
\end{array}$ & $\begin{array}{l}4,1,26,2,15 \\
1,0,7,0,0 \\
4,1,43,3,7 \\
\end{array}$ & $\begin{array}{l}5,4,21,5,18 \\
1,0,4,0,0 \\
14,5,75,6,19 \\
\end{array}$ & $\begin{array}{l}17,12,47,15,38 \\
1,0,2,0,0 \\
17,15,116,16,54 \\
\end{array}$ & $\begin{array}{l}16,8,36,16,18 \\
1,0,4,0,0 \\
50,14,79,25,51 \\
\end{array}$ & $\begin{array}{l}7,4,18,6,9 \\
0,0,1,0,0 \\
12,5,19,9,17 \\
\end{array}$ & $\begin{array}{l}0,0,1,0,0 \\
0,0,0,0,0 \\
9,0,32,3,10 \\
\end{array}$ \\
\hline Epiglobulol & $\begin{array}{l}0,0,0,0,0 \\
0,0,0,0,0 \\
0,0,0,0,0\end{array}$ & $\begin{array}{l}0,0,0,0,0 \\
0,0,0,0,0 \\
0,0,0,0,0\end{array}$ & $\begin{array}{l}0,0,5,0,3 \\
0,0,0,0,0 \\
0,0,3,0,1\end{array}$ & $\begin{array}{l}1,1,9,1,2 \\
0,0,0,0,0 \\
0,0,10,0,1\end{array}$ & $\begin{array}{l}4,2,5,2,4 \\
0,0,0,0,0 \\
2,0,18,0,10\end{array}$ & $\begin{array}{l}2,1,2,1,2 \\
0,0,0,0,0 \\
4,1,7,1,5\end{array}$ & $\begin{array}{l}0,0,1,0,0 \\
0,0,0,0,0 \\
0,0,2,0,0\end{array}$ & $\begin{array}{l}0,0,0,0,0 \\
0,0,0,0,0 \\
0,0,1,0,0\end{array}$ \\
\hline
\end{tabular}




\begin{tabular}{|c|c|c|c|c|c|c|c|c|}
\hline \multirow{2}{*}{ Compound } & $25^{\circ} \mathrm{C}$ & $50^{\circ} \mathrm{C}$ & $75^{\circ} \mathrm{C}$ & $100^{\circ} \mathrm{C}$ & $150^{\circ} \mathrm{C}$ & $200^{\circ} \mathrm{C}$ & $250^{\circ} \mathrm{C}$ & $300^{\circ} \mathrm{C}$ \\
\hline & \multicolumn{5}{|c|}{ Fresh stage } & Heated stage & Prefiring stage & Post-Ignition \\
\hline \multicolumn{9}{|l|}{ Furanes } \\
\hline Furfural & $\begin{array}{l}0,0,0,0,0 \\
0,0,0,0,0 \\
0,0,0,0,0\end{array}$ & $\begin{array}{l}0,0,0,0,0 \\
0,0,0,0,0 \\
0,0,0,0,0\end{array}$ & $\begin{array}{l}0,0,0,0,0 \\
0,0,0,0,0 \\
0,0,0,0,0\end{array}$ & $\begin{array}{l}0,0,0,0,0 \\
0,0,0,0,0 \\
0,0,0,0,0\end{array}$ & $\begin{array}{l}0,0,0,0,0 \\
0,0,0,0,0 \\
0,0,2,0,0\end{array}$ & $\begin{array}{l}5,1,11,3,8 \\
55,4,129,39,61 \\
35,9,42,31,35\end{array}$ & $\begin{array}{l}89,25,312,68,110 \\
514,64,810,488,669 \\
68,14,162,58,77\end{array}$ & $\begin{array}{l}4,2,7,3,7 \\
13,2,44,4,10 \\
36,6,56,20,38\end{array}$ \\
\hline Compound & $25^{\circ} \mathrm{C}$ & $50^{\circ} \mathrm{C}$ & $75^{\circ} \mathrm{C}$ & $100^{\circ} \mathrm{C}$ & $150^{\circ} \mathrm{C}$ & $200^{\circ} \mathrm{C}$ & $250^{\circ} \mathrm{C}$ & $300{ }^{\circ} \mathrm{C}$ \\
\hline 2-Furanmethanol & $\begin{array}{l}0,0,0,0,0 \\
0,0,0,0,0 \\
0,0,0,0,0\end{array}$ & $\begin{array}{l}0,0,0,0,0 \\
0,0,0,0,0 \\
0,0,0,0,0\end{array}$ & $\begin{array}{l}0,0,0,0,0 \\
0,0,0,0,0 \\
0,0,0,0,0\end{array}$ & $\begin{array}{l}0,0,0,0,0 \\
0,0,0,0,0 \\
0,0,0,0,0\end{array}$ & $\begin{array}{l}0,0,0,0,0 \\
0,0,0,0,0 \\
0,0,0,0,0\end{array}$ & $\begin{array}{l}5,1,12,3,8 \\
8,0,16,4,14 \\
0,0,0,0,0\end{array}$ & $\begin{array}{l}89,25,312,68,111 \\
100,3,263,18,133 \\
0,0,0,0,0\end{array}$ & $\begin{array}{l}4,2,7,3,7 \\
41,0,101,4,70 \\
0,0,0,0,0\end{array}$ \\
\hline 5-Methyl-2(5)-Furanone & $\begin{array}{l}0,0,0,0,0 \\
0,0,0,0,0 \\
0,0,0,0,0 \\
\end{array}$ & $\begin{array}{l}0,0,0,0,0 \\
0,0,0,0,0 \\
0,0,0,0,0 \\
\end{array}$ & $\begin{array}{l}0,0,0,0,0 \\
0,0,0,0,0 \\
0,0,0,0,0 \\
\end{array}$ & $\begin{array}{l}0,0,0,0,0 \\
0,0,0,0,0 \\
0,0,0,0,0 \\
\end{array}$ & $\begin{array}{l}0,0,0,0,0 \\
0,0,0,0,0 \\
0,0,0,0,0 \\
\end{array}$ & $\begin{array}{l}0,0,0,0,0 \\
0,0,0,0,0 \\
0,0,0,0,0 \\
\end{array}$ & $\begin{array}{l}1,0,2,1,2 \\
2,0,7,0,4 \\
0,0,0,0,0 \\
\end{array}$ & $\begin{array}{l}0,0,0,0,0 \\
1,0,3,0,0 \\
0,0,0,0,0 \\
\end{array}$ \\
\hline 5-Methylfurfural & $\begin{array}{l}0,0,0,0,0 \\
0,0,0,0,0 \\
0,0,0,0,0 \\
\end{array}$ & $\begin{array}{l}0,0,0,0,0 \\
0,0,0,0,0 \\
0,0,0,0,0 \\
\end{array}$ & $\begin{array}{l}0,0,0,0,0 \\
0,0,0,0,0 \\
0,0,0,0,0 \\
\end{array}$ & $\begin{array}{l}0,0,0,0,0 \\
0,0,0,0,0 \\
0,0,0,0,0 \\
\end{array}$ & $\begin{array}{l}0,0,0,0,0 \\
0,0,0,0,0 \\
0,0,0,0,0 \\
\end{array}$ & $\begin{array}{l}3,2,6,2,5 \\
4,0,10,3,6 \\
5,1,6,4,6 \\
\end{array}$ & $\begin{array}{l}22,15,31,20,24 \\
23,4,35,17,29 \\
8,3,15,8,8\end{array}$ & $\begin{array}{l}0,0,0,0,0 \\
3,0,6,0,5 \\
5,1,10,2,7\end{array}$ \\
\hline 5-Hydroxymethylfurfural & $\begin{array}{l}0,0,0,0,0 \\
0,0,0,0,0 \\
0,0,0,0,0\end{array}$ & $\begin{array}{l}0,0,0,0,0 \\
0,0,0,0,0 \\
0,0,0,0,0\end{array}$ & $\begin{array}{l}0,0,0,0,0 \\
0,0,0,0,0 \\
0,0,0,0,0\end{array}$ & $\begin{array}{l}0,0,0,0,0 \\
0,0,0,0,0 \\
0,0,0,0,0\end{array}$ & $\begin{array}{l}0,0,0,0,0 \\
0,0,0,0,0 \\
0,0,0,0,0\end{array}$ & $\begin{array}{l}7,4,10,6,9 \\
30,0,56,12,44 \\
0,0,15,0,0\end{array}$ & $\begin{array}{l}0,0,0,0,0 \\
0,0,0,0,0 \\
0,0,2,0,0\end{array}$ & $\begin{array}{l}0,0,0,0,0 \\
10,0,39,0,11 \\
0,0,2,0,0\end{array}$ \\
\hline \multicolumn{9}{|l|}{ Methoxyphenols } \\
\hline 2-Methoxyphenol & $\begin{array}{l}0,0,0,0,0 \\
0,0,0,0,0 \\
0,0,0,0,0\end{array}$ & $\begin{array}{l}0,0,0,0,0 \\
0,0,0,0,0 \\
0,0,0,0,0\end{array}$ & $\begin{array}{l}0,0,6,0,1 \\
0,0,0,0,0 \\
0,0,0,0,0 \\
\end{array}$ & $\begin{array}{l}0,0,6,0,0 \\
0,0,0,0,0 \\
0,0,0,0,0\end{array}$ & $\begin{array}{l}0,0,12,0,7 \\
0,0,0,0,0 \\
0,0,0,0,0\end{array}$ & $\begin{array}{l}5,1,14,4,8 \\
0,0,0,0,0 \\
0,0,0,0,0 \\
\end{array}$ & $\begin{array}{l}9,5,12,5,9 \\
11,1,22,6,18 \\
2,1,3,1,2 \\
\end{array}$ & $\begin{array}{l}0,0,2,0,1 \\
14,1,45,3,15 \\
5,1,16,4,5 \\
\end{array}$ \\
\hline $\begin{array}{l}\text { 4-Methyl-2- } \\
\text { methoxyphenol }\end{array}$ & $\begin{array}{l}0,0,0,0,0 \\
0,0,0,0,0 \\
0,0,0,0,0 \\
\end{array}$ & $\begin{array}{l}0,0,0,0,0 \\
0,0,0,0,0 \\
0,0,0,0,0 \\
\end{array}$ & $\begin{array}{l}0,0,0,0,0 \\
0,0,0,0,0 \\
0,0,0,0,0 \\
\end{array}$ & $\begin{array}{l}0,0,0,0,0 \\
0,0,0,0,0 \\
0,0,0,0,0 \\
\end{array}$ & $\begin{array}{l}0,0,0,0,0 \\
0,0,0,0,0 \\
0,0,0,0,0 \\
\end{array}$ & $\begin{array}{l}0,0,0,0,0 \\
0,0,0,0,0 \\
0,0,0,0,0 \\
\end{array}$ & $\begin{array}{l}4,3,5,3,4 \\
4,1,6,3,6 \\
0,0,0,0,0 \\
\end{array}$ & $\begin{array}{l}2,0,3,1,3 \\
4,0,11,1,6 \\
1,0,3,0,1 \\
\end{array}$ \\
\hline 4-Ethyl-2-methoxyphenol & $\begin{array}{l}0,0,0,0,0 \\
0,0,0,0,0 \\
0,0,0,0,0 \\
\end{array}$ & $\begin{array}{l}0,0,0,0,0 \\
0,0,0,0,0 \\
0,0,0,0,0 \\
\end{array}$ & $\begin{array}{l}0,0,0,0,0 \\
0,0,0,0,0 \\
0,0,0,0,0 \\
\end{array}$ & $\begin{array}{l}0,0,0,0,0 \\
0,0,0,0,0 \\
0,0,0,0,0 \\
\end{array}$ & $\begin{array}{l}0,0,0,0,0 \\
0,0,0,0,0 \\
0,0,0,0,0 \\
\end{array}$ & $\begin{array}{l}0,0,0,0,0 \\
0,0,0,0,0 \\
0,0,0,0,0 \\
\end{array}$ & $\begin{array}{l}0,0,0,0,0 \\
0,0,0,0,0 \\
0,0,0,0,0 \\
\end{array}$ & $\begin{array}{l}1,0,3,0,1 \\
1,0,4,0,2 \\
1,0,4,1,1 \\
\end{array}$ \\
\hline $\begin{array}{l}\text { 2-Methoxy-4-(2)- } \\
\text { propenylphenol }\end{array}$ & $\begin{array}{l}0,0,0,0,0 \\
0,0,0,0,0 \\
0,0,0,0,0 \\
\end{array}$ & $\begin{array}{l}0,0,0,0,0 \\
0,0,0,0,0 \\
0,0,0,0,0 \\
\end{array}$ & $\begin{array}{l}0,0,0,0,0 \\
0,0,0,0,0 \\
0,0,0,0,0 \\
\end{array}$ & $\begin{array}{l}0,0,0,0,0 \\
0,0,0,0,0 \\
0,0,0,0,0 \\
\end{array}$ & $\begin{array}{l}0,0,0,0,0 \\
0,0,0,0,0 \\
0,0,0,0,0 \\
\end{array}$ & $\begin{array}{l}0,0,0,0,0 \\
0,0,0,0,0 \\
0,0,0,0,0 \\
\end{array}$ & $\begin{array}{l}1,0,1,0,1 \\
1,0,1,1,1 \\
0,0,0,0,0 \\
\end{array}$ & $\begin{array}{l}0,0,0,0,0 \\
0,0,2,0,1 \\
0,0,0,0,0 \\
\end{array}$ \\
\hline $\begin{array}{l}\text { 2-Methoxy-4- } \\
\text { formylphenol }\end{array}$ & $\begin{array}{l}0,0,0,0,0 \\
0,0,0,0,0 \\
0,0,0,0,0\end{array}$ & $\begin{array}{l}0,0,0,0,0 \\
0,0,0,0,0 \\
0,0,0,0,0\end{array}$ & $\begin{array}{l}0,0,0,0,0 \\
0,0,0,0,0 \\
0,0,0,0,0\end{array}$ & $\begin{array}{l}0,0,0,0,0 \\
0,0,0,0,0 \\
0,0,0,0,0\end{array}$ & $\begin{array}{l}0,0,0,0,0 \\
0,0,0,0,0 \\
0,0,0,0,0\end{array}$ & $\begin{array}{l}0,0,0,0,0 \\
1,0,3,0,1 \\
0,0,0,0,0\end{array}$ & $\begin{array}{l}7,5,11,6,10 \\
10,0,20,8,13 \\
0,0,1,0,0\end{array}$ & $\begin{array}{l}1,0,2,1,1 \\
9,0,20,0,16 \\
1,0,5,0,2\end{array}$ \\
\hline \multicolumn{9}{|l|}{ Benzenes } \\
\hline $\begin{array}{l}\text { 1-Isopropyl-4- } \\
\text { methylbenzene }\end{array}$ & $\begin{array}{l}0,0,2,0,0 \\
0,0,0,0,0 \\
0,0,1,0,1\end{array}$ & $\begin{array}{l}0,0,2,0,0 \\
0,0,1,0,0 \\
0,0,1,0,0\end{array}$ & $\begin{array}{l}1,0,1,0,1 \\
0,0,0,0,0 \\
0,0,2,0,0\end{array}$ & $\begin{array}{l}1,0,2,0,1 \\
0,0,1,0,0 \\
0,0,1,0,0\end{array}$ & $\begin{array}{l}2,1,9,2,4 \\
0,0,2,0,0 \\
0,0,3,0,0\end{array}$ & $\begin{array}{l}4,4,17,4,11 \\
0,0,2,0,0 \\
4,0,22,0,13\end{array}$ & $\begin{array}{l}15,8,26,12,19 \\
0,0,1,0,0 \\
18,3,64,11,24\end{array}$ & $\begin{array}{l}0,0,1,0,1 \\
0,0,0,0,0 \\
31,17,55,22,52\end{array}$ \\
\hline
\end{tabular}




\subsubsection{EAG with M. atrata}

Thirteen of the 20 compounds tested on the antenna of $M$. atrata showed a significant response (Table 3). Among them were eight terpenes ( $\alpha$-Pinene, camphene, $\beta$-pinene, $\beta$-myrcene, limonene, eucalyptol, camphor, and epiglobulol), three furans (furfural, 2-furanmethanol, 5-methylfurfural), one methoxyphenol (2-methoxyphenol) and one benzene (1-isopropyl-4-methylbenzene). The calculated vapor pressure of the first significant concentration varied between $0.179 \times 10^{-4}$ for 2 methoxyphenol and $146 \times 10^{-4}$ for 1-Isopropyl-4-methylbenzene. 2-Methoxyphenol, $\alpha$-pinene, epiglobulol, $\beta$-myrcen, limonene, eucalyptol, furfural, and 2-furanmethanol show a vapor pressure of the first significant response below three. Aromadendrene, 5-hydroxymethylfurfural, 4-methyl-2methoxyphenol, 4-ethylguaiacol, 2-methoxy-4-(2)-propenylphenol, and 2-methoxy-4-formylphenol showed no significant response at a concentration of $10^{-2} \mathrm{~g} / \mathrm{g}$ in paraffin oil in the EAG studies.

\subsection{Discussion}

\subsubsection{Volatile emission from thermally oxidized $E$. globulus tissues}

The emissions of the wood and the leaves of E. globulus show a pattern correlated to the temperature. The general tendency is the succession of terpenes, furans, and finally methoxyphenols. The emission of terpenes mainly derive from the leaves (Maleknia et al., 2009), as the highest terpene emissions are found in the leave and in the wood and leave samples (Table 2). While most terpenes are emitted at low concentrations, $\alpha$-pinene and eucalyptol are emitted reliably at high concentrations. $\alpha$-Pinene is emitted from $25^{\circ} \mathrm{C}$ to $250^{\circ} \mathrm{C}$ with a maximum emission at $150^{\circ} \mathrm{C}$ (fresh stage). Eucalyptol is emitted throughout the heating process with a maximum emission at $200^{\circ} \mathrm{C}$ (heated stage). Both compounds do not form from combustion processes, but are ingredients of eucalypt leaves and, to a lower extent, of eucalypt wood. Camphene might form by secondary chemical reactions, which are induced by high temperatures (Neuenschwander et al., 2010), because it is emitted in the temperature range between $200^{\circ} \mathrm{C}$ and $300^{\circ} \mathrm{C}$. Epiglobulol, which is an ingredient of eucalypt leaves (Chalchat et al., 1995), is mainly emitted in an intermediate temperature range at low concentrations because of its low vapor pressure. $\beta$-Pinene, $\beta$-myrcen, and camphor show very low emission rates and, therefore, are irrelevant for an early fire warning sensor concept.

At $200^{\circ} \mathrm{C}$ the decomposition of hemicelluloses and cellulose lead to furan emissions (RisholmSudman et al., 1998; Fengel and Wegener, 1984; Oasmaa et al., 2003; Tjeerdsma et al., 1998; Chheda et al., 2006; Browne, 1958)(Table 3, this thesis p.101). The furans emitted with the highest quantity are furfural and 2-furanmethanol, which start to form at $200^{\circ} \mathrm{C}$ (heated stage) and reach their maximum emission at $250^{\circ} \mathrm{C}$ (preignition stage)(Table 2).

In the heated stage the combustion of the macromolecule lignin leads to an increasing formation of methoxyphenols (Azeez et al., 2010; Branca et al., 2006; Ingram et al., 2008; Karagoz et al., 2005; Greenberg et al., 2006) (Table 3, this thesis p.101). These volatile compounds predominantly form from the combustion of the lignin-rich wood tissue, but can be found at lower concentrations in the leave samples, which also contain lignin (Table 2). 
Table 3: EAG. The table shows the antennal responses of $M$. atrata towards the identified and quantified volatile combustion products identified in the thermal oxisation experiments with $E$. globulus wood, leaves, and wood and leaves. The responses in $\mathrm{mV}$ were described in the following order by median, minimum, maximum, and upper and lower quartiles. Significant differences between the paraffin oil controls and the compound dilutions is indicated by stars $(p<0,05=* ; p<0,01=* * ; p<0,001=* * *)$. A low vapor pressure of the $1^{\text {st }}$ significant concentration indicates a low detection threshold and a (-) shows that the compound was not detected.

\begin{tabular}{|c|c|c|c|c|c|c|c|c|}
\hline Compound & Paraffin oil & $10^{-7}$ & $10^{-6}$ & $10^{-5}$ & $10^{-4}$ & $10^{-3}$ & $10^{-2}$ & $\begin{array}{c}\text { Vapor pressure } \\
\text { of } 1^{\text {st }} \text { significant } \\
\text { concentration } \\
{\left[\mathrm{hPa} \times 10^{4}\right]}\end{array}$ \\
\hline \multicolumn{9}{|l|}{ Terpenes } \\
\hline$\alpha$-Pinene & $30,9,62,11,45$ & $19,14,74,16,48$ & $32,22,113,22,50$ & $\begin{array}{c}* * \\
62,39,147,53,79\end{array}$ & $\begin{array}{c}* * \\
75,42,237,71,105\end{array}$ & $\begin{array}{c}* * \\
87,44,290,83,132\end{array}$ & $\begin{array}{c}* * \\
110,43,361,91,156\end{array}$ & 0.349 \\
\hline Camphene & $19,8,31,15,20$ & $18,12,30,14,18$ & $21,14,26,20,25$ & $26,17,39,23,30$ & $\begin{array}{c}* * \\
40,30,63,37,48 \\
\end{array}$ & $\begin{array}{c}* * \\
59,35,85,48,70 \\
\end{array}$ & $\begin{array}{c}* * * \\
77,44,90,53,89\end{array}$ & 3.38 \\
\hline$\beta$-Pinene & $25,15,30,23,29$ & $26,22,30,22,29$ & $26,21,32,24,29$ & $30,27,42,29,35$ & $\begin{array}{c}* * \\
45,37,54,38,48\end{array}$ & $\begin{array}{c}* * * \\
64,46,85,52,70\end{array}$ & $\begin{array}{c}* * \\
79,47,119,76,79\end{array}$ & 24 \\
\hline$\beta$-Myrcene & $9,4,17,7,15$ & $14,7,18,9,17$ & $15,9,17,9,16$ & $16,10,23,11,16$ & $\begin{array}{c}* * \\
17,14,26,15,24 \\
\end{array}$ & $\begin{array}{c}* * \\
25,21,40,23,33 \\
\end{array}$ & $\begin{array}{c}* * \\
45,37,60,42,48 \\
\end{array}$ & 2.29 \\
\hline Limonene & $25,15,30,23,29$ & $33,18,45,22,37$ & $29,19,46,24,41$ & $31,23,46,30,35$ & $\begin{array}{c}* * \\
41,34,57,37,45\end{array}$ & $\begin{array}{c}* * * \\
70,53,77,62,75\end{array}$ & $\begin{array}{c}* * * \\
111,77,14092,117\end{array}$ & 1.54 \\
\hline Eucalyptol & $16,9,21,15,18$ & $17,12,61,17,4 ß$ & $22,15,50,18,42$ & $25,14,68,22,43$ & $\begin{array}{c}* * \\
35,19,69,28,42\end{array}$ & $\begin{array}{c}* * * \\
54,40,72,45,58\end{array}$ & $\begin{array}{c}* * * \\
81,70,86,70,83\end{array}$ & 1.9 \\
\hline Camphor & $9,4,17,7,15$ & $8,6,16,8,15$ & $9,8,19,8,16$ & $18,9,23,14,20$ & $\begin{array}{c}* * \\
27,21,39,25,31\end{array}$ & $\begin{array}{c}* * * \\
36,29,48,35,41\end{array}$ & $\begin{array}{c}* * \\
48,44,63,46,51\end{array}$ & 4 \\
\hline Aromadendrene & $15,9,19,15,17$ & $15,12,19,14,17$ & $15,13,17,14,17$ & $15,13,18,15,17$ & $15,13,18,14,18$ & $17,14,20,15,19$ & $17,15,22,16,21$ & - \\
\hline Epiglobulol & $15,8,19,15,17$ & $16,12,22,15,17$ & $16,12,24,16,17$ & $15,13,25,15,18$ & $16,13,23,15,18$ & $\begin{array}{c}* * \\
20,18,25,19,23\end{array}$ & $\begin{array}{c}* * \\
26,21,28,22,27\end{array}$ & 0.3 \\
\hline \multicolumn{9}{|l|}{ Furanes } \\
\hline Furfural & $19,10,31,16,25$ & $29,12,76,24,32$ & $26,18,73,23,32$ & $28,20,73,27,33$ & $\begin{array}{c}* \\
34,28,79,32,40\end{array}$ & $\begin{array}{c}* * \\
49,42,82,48,65\end{array}$ & $\begin{array}{c}* * * \\
64,48,86,59,81\end{array}$ & 2.23 \\
\hline 2-Furanmethanol & $9,4,17,7,15$ & $15,6,19,8,16$ & $18,8,26,8,22$ & $21,10,32,13,25$ & $\begin{array}{c}* * \\
30,16,40,29,35 \\
\end{array}$ & $\begin{array}{c}* * * \\
48,33,64,43,51 \\
\end{array}$ & $\begin{array}{c}* * * \\
63,45,110,58,85 \\
\end{array}$ & 1.009 \\
\hline 5-Methyl-2(5)-Furanone & $15,12,22,14,15$ & $17,15,32,16,18$ & $18,15,26,16,19$ & $19,15,22,15,21$ & $17,14,22,14,18$ & $15,13,19,14,18$ & $16,12,22,14,18$ & - \\
\hline
\end{tabular}




\begin{tabular}{|c|c|c|c|c|c|c|c|c|}
\hline Compound & Paraffin oil & $10^{-7}$ & $10^{-6}$ & $10^{-5}$ & $10^{-4}$ & $10^{-3}$ & $10^{-2}$ & $\begin{array}{c}\text { Vapor pressure } \\
\text { of } 1^{\text {st }} \text { significant } \\
\text { concentration } \\
{\left[\mathrm{hPa} \times 10^{4}\right]}\end{array}$ \\
\hline 5-Methylfurfural & $20,9,26,12,21$ & $22,12,45,12,23$ & $23,12,36,13,25$ & $13,11,35,12,25$ & $15,12,30,13,24$ & $18,16,33,16,30$ & $\begin{array}{c}* * \\
38,17,49,34,45\end{array}$ & 64.4 \\
\hline 5-Hydroxymethylfurfural & $19,11,26,16,20$ & $18,12,25,15,20$ & $15,15,25,15,20$ & $14,10,32,12,21$ & $17,9,32,12,24$ & $21,9,33,12,23$ & $19,6,42,14,26$ & - \\
\hline \multicolumn{9}{|l|}{ Methoxyphenols } \\
\hline 2-Methoxyphenol & $19,16,61,18,21$ & $30,14,87,23,66$ & $44,18,86,23,73$ & $40,18,73,26,72$ & $\begin{array}{c}* \\
38,24,77,26,74\end{array}$ & $\begin{array}{c}* * \\
42,34,113,34,78\end{array}$ & $\begin{array}{c}* * * \\
85,50,176,67,111\end{array}$ & 0.179 \\
\hline $\begin{array}{l}\text { 4-Methyl-2- } \\
\text { methoxyphenol }\end{array}$ & $16,9,31,12,26$ & $19,6,28,12,19$ & $18,8,30,12,25$ & $14,7,31,14,23$ & $13,9,29,13,24$ & $17,9,34,15,27$ & $34,18,52,26,39$ & - \\
\hline 4-Ethyl-2-methoxyphenol & $15,10,26,11,23$ & $12,9,26,10,19$ & $15,11,30,11,24$ & $14,9,31,11,23$ & $13,9,27,10,21$ & $16,11,30,12,25$ & $20,14,43,15,33$ & - \\
\hline $\begin{array}{l}\text { 2-Methoxy-4-(2)- } \\
\text { propenylphenol }\end{array}$ & $23,11,37,16,31$ & $18,10,64,12,24$ & $19,9,70,11,32$ & $15,11,66,12,34$ & $19,10,60,13,24$ & $19,11,76,13,33$ & $23,13,84,14,36$ & - \\
\hline $\begin{array}{l}\text { 2-Methoxy-4- } \\
\text { formylphenol }\end{array}$ & $18,9,21,10,20$ & $17,11,29,13,22$ & $20,12,23,14,21$ & $19,12,25,13,24$ & $20,12,27,12,25$ & $14,11,25,13,20$ & $12,9,26,10,25$ & - \\
\hline \multicolumn{9}{|l|}{ Benzenes } \\
\hline $\begin{array}{l}\text { 1-Isopropyl-4- } \\
\text { methylbenzene }\end{array}$ & $15,9,19,15,17$ & $16,10,27,15,18$ & $17,12,25,15,18$ & $17,12,25,13,18$ & $16,12,24,13,18$ & $22,15,29,17,23$ & $\begin{array}{c}* * \\
38,24,41,27,40\end{array}$ & 146 \\
\hline
\end{tabular}




\subsubsection{Evaluation of marker volatiles for early fire warning}

According to the hypothesis of marker volatiles for early fire warning, volatiles should be selected, which fulfill the three requirements for marker volatiles (1: reliable emission prior to ignition; 2: characteristic for heated wood; 3: high concentration). According to the vapor pressure of the first significant electrophysiological active concentration, $\alpha$-pinene, limonene, eucalyptol, furfural, 2furanmethanol, and 2-methoxyphenol are detected by M. atrata with high sensitivity (vapor pressure of the first significant concentration below $3 \mathrm{hPa} \times 10^{4}$ ). These marker compound candidates can be regarded as reliably associated with the thermal oxidation of eucalypt wood and leaves, because the beetle adapted during millions of years of evolution to their perception due to the law of the survival of the fittest (Darwin, 1859). All of these compounds are emitted in the fresh, heated, or preignition stage and, therefore, they fulfill the first criteria of marker volatiles for the prediction of woodland fires.

All of these compounds are emitted by different heated woods or heated leaves (Risholm-Sudman et al., 1998; Fengel and Wegener, 1984; Oasmaa et al., 2003; Tjeerdsma et al., 1998; Chheda et al., 2006; Browne, 1958; Maleknia et al., 2009; Neuenschwander et al., 2010). Only eucalyptol is a compound emitted at higher quantities from eucalypt leaves than from other tree species. Therefore, most of the compounds detected by $M$. atrata are general thermal oxidation products and can be regarded as typical marker for most species of heated wood and leaves. In a forest habitat, there are no natural sources that might emit these compounds in large quantities, which would lead to false alarms. A rising ambient temperature might lead to an increasing eucalyptol emission, although there is no early stage fire. Furfural forms during the cooking of meat (Meynier and Mottram, 2000), but this will not lead to false alarms under field conditions and a decaying vertebrate cadaver in a wood storage will not irritate a sensor system tuned to the detection of furfural (Paczkowski and Schütz, 2011). Some fungi emit low concentrations of furfural (Sunesson et al., 1996) and the compound can be emitted by soil and litter (Leff and Fierer, 2008), which might irritate a sensor in a forest habitat. However, the concentrations emitted by heated wood are several orders of magnitude higher. All of the compounds sensitively perceived by M.atrata fulfill the second criterion of marker volatiles.

The third criterion is the emission of high quantities. Among the compounds that fulfill the first and the second criterion, $\alpha$-pinene, eucalyptol, furfural and furanmethanol are emitted at high concentrations prior to ignition. The first sign of heating is an increasing emission of $\alpha$-pinen and eucalyptol from the leaves of $\mathrm{E}$. globulus. In the temperature range from $25^{\circ} \mathrm{C}$ to $150^{\circ} \mathrm{C}$ the median of the $\alpha$-pinen emission from E. globulus leaves increases from $12 \mathrm{ng} / \mathrm{L}$ to $94 \mathrm{ng} / \mathrm{L}$ and the median of eucalyptol increases from $23 \mathrm{ng} / \mathrm{L}$ to $250 \mathrm{ng} / \mathrm{L}$. At $200^{\circ} \mathrm{C}$, in the heated stage, furfural and 2furanmethanol are emitted from the cellulose and hemicellulose of the combusting wood samples (Table 3, p.101). The median of their emission strongly increases from 55 to $514 \mathrm{ng} / \mathrm{L}$ (furfural) and from 8 to $100 \mathrm{ng} / \mathrm{L}$ (2-furanmethanol) in the preignition stage.

According to these results, an increasing terpene emission is a reliable sign for heated wood and an increasing furan emission is a reliable sign for a high fire risk. 2-Methoxyphenol can serve as a compound for improved fire detection, but it is emitted at low quantities prior to ignition and cannot serve as a marker for early fire warning. It should be remarked that the reason for the relatively low 
emissions of methoxyphenols is that the smoke itself was not sampled in this experiment. Semi conductor gas sensors were already adapted to the detection of $\alpha$-pinen and furfural for the heat stage assessment of Pinus sylvestris wood flakes in wood flake dryers of the wood processing industry (Eßinger et al., 2010; Paczkowski et al., 2011). As gas sensors yet require AC mains power, their application will be restricted to punctual monitoring of farm houses or village houses. However, such systems can increase the fire safety, as they will be able to predict fires before they ignite. Further enhancement of sensor selectivity and field test with an early fire warning gas sensor system should show the practicability of this approach toward wood land fire safety.

\subsubsection{Summary}

The aim of this study was the utilization of the olfaction of $M$. atrata for the selection of marker volatiles for gas sensor systems that can predict fires before ignition. Trace analytical results from wood, leaves, and wood and leaves at increasing temperatures were compared with the sensitivity of M. atrata towards the identified and quantified compounds. The emission of terpenes, furfural and 2-methoxyphenol are correlated to the heating stages of the tissue and are perceived by the beetles. Therefore, these compound groups / compounds have the potential to enable sensor systems to predict woodland fires.

\subsubsection{Acknowledgements}

We like to thank David Lindgen for his technical assistance during the trace analytical and electrophyiological experiments. We further like to thank the botanical garden in Bremen, Germany, the botanical garden of the Phillips University in Marburg, Germany, the botanical garden of the University in Leipzig, Germany, the botanical garden of the Martin-Luther University in Halle, Germany, and the botanical garden of the Ruhr University in Bochum, Germany, for providing the $E$. globulus branches.

\subsubsection{References}

Azeez,A.M., Meier,D., Odermatt,J. and Willner,T. (2010) Fast pyrolysis of African and European lignocellulosic biomasses using Py-GC/MS and fluidized bed reactor. Energy \& Fuels, 24, 2078-2085.

Branca,C., Di Blasi,C. and Elefante,R. (2006) Devolatilization of conventional pyrolysis oils generated from biomass and cellulose. Energy \& Fuels, 20, 2253-2261.

Browne, F. L. (1958) Theories of the combustion of wood and its control: A literature survey. 2136, 159. U.S. Department of Agriculture. Forest Products Laboratory.

Castello,G., Moretti,P. and Vezzani,S. (2009) Retention models for programmed gas chromatography. Journal of Chromatography A, 1216, 1607-1623.

Chalchat,J.-C., Cabard,J.L., Gorunovic,M.S., Djermanovic,V. and Bulatovic,V. (1995) Chemical composition of Eucalyptus globulus oils from the Montenegro coast and east coast of spain. Journal of Essential Oil Research, 7, 147-152. 
Chheda,J.N., Roman-Leshkov,Y. and Dumesic,J.A. (2006) Production of 5-hydroxymethylfurfural and furfural by dehydration of biomass-derived mono- and poly-saccharides. Green Chemistry, 9, 342350.

Darwin,C. (1859) On the origin of species by means of natural selection, or the preservation of favoured races in the struggle for live. Oxford University Press, Oxford, United Kingdom.

Eßinger, T. M., Becker, B., Sauerwald, T., Kohl, C.-D., Paczkowski, S., and Schütz, S. (2010) Volatile gas sensors for large-scale drying of wood. Conference Proceedings: Sensoren und Messsysteme 2010 - 15. ITG/GMA-Fachtagung, Nürnberg, VDE-Verlag, Berlin, Germany.

Evans,W.G. (2010) Reproductive role of infrared radiation sensors of Melanophila acuminata (Coleoptera, Buprestidae) at forest fires. Annals of the Entomological Society of America, 103, 823826.

Fengel,D. and Wegener,G. (1984) Wood: Chemistry, ultrastructure, reactions. De Gruyter Inc., Boston, USA.

Greenberg,J.P., Friedli,H., Guenther,A.B., Hanson,D., Harley,P. and Karl,T. (2006) Volatile organic emissions from the distillation and pyrolysis of vegetation. Athmospheric Chemistry and Physics, 6 , 81-91.

Ingram,L., Mohan,D., Bricka,M., Steele,P., Strobel,D., Crocker,D., Mitchell,B., Mohammad,J., Cantrell,K. and Pittman,C.U. (2008) Pyrolysis of wood and bark in an auger reactor: Physical properties and chemical analysis of the produced bio-oils. Energy \& Fuels, 22, 614-625.

Karagoz,S., Bhaskar,T., Muto,A. and Sakata,Y. (2005) Comparative studies of oil compositions produced from sawdust, rice husk, lignin and cellulose by hydrothermal treatment. Fuel, 84, 875-884.

Kohl,C.-D. (2003) Electronic Noses. In Waser,R. (ed.), Nanoelectronics and Information Technology Advanced Electronic Materials and Novel Devices. Wiley-VCH, Berlin.

Kreiss,E., Schmitz,H. and Gebhardt,M. (2007) Electrophysiological characterisation of the infrared organ of the Australian "Little Ash Beetle" Acanthocnemus nigricans (Coleoptera, Acanthocnemidae). Journal of Comparative Physiology A-Neuroethology Sensory Neural and Behavioral Physiology, 193, 729-739.

Leff,J.W. and Fierer,N. (2008) Volatile organic compound (VOC) emissions from soil and litter samples. Soil Biology and Biochemistry, 40, 1629-1636.

Maleknia,S.D., Bell,T.L. and Adams,M.A. (2009) Eucalypt smoke and wildfires: Temperature dependent emissions of biogenic volatile organic compounds. International Journal of Mass Spectrometry, 279, 126-133.

Meynier, A. and Mottram, D. S. (2000) The effect of $\mathrm{pH}$ on the formation of volatile compounds in meat-related model systems. Food Chemistry 52, 361-366. 2000.

Neuenschwander,U., Guignard,F. and Hermans,I. (2010) Mechanism of the aerobic oxidation of $\alpha$ pinene. ChemSusChem, 3, 75-84.

Oasmaa,A., Kuoppala,E. and Solantausta,Y. (2003) Fast pyrolysis of forestry residue. 2. Physicochemical composition of product liquid. Energy \& Fuels, 17, 433-443. 
Paczkowski, S. and Schütz, S. (2011) Post-mortem volatiles of vertebrate decay. Applied Microbiology and Biotechnology 91, 917-935. 2011.

Paczkowski, S., Sauerwald, T., Weiß.A., Bauer, M., Kohl, C.-D., and Schütz, S. (2011) Biomimetic gas sensors for large-scale drying of wood particles. Martin-Palma, R. J. and Lakhtakia, A. 7975, 797505797505-8. Conference Proceedings SPIE: Bioinspiration, Biomimetics, and Bioreplication, San Diego, USA.

Paczkowski, S., Paczkowska, M., Maibaum, F., and Schütz, S. (2012) Decaying mouse volatiles perceived by Calliphora vicina Rob.-Desv. Journal of Forensic Sciences. 57, 1497-1506.

Pelosi,P. and Maida,R. (1995) Odorant-binding proteins in insects. Comparative Biochemistry and Physiology B-Biochemistry \& Molecular Biology, 111, 503-514.

Risholm-Sudman,M., Lundgren,M., Vestin,E. and Herder,P. (1998) Emissions of acetic acid and other volatile organic compounds from different species of solid wood. Holz als Roh- und Werkstoff, 56, 125-129.

Schmitz,H., Bleckmann,H. and Murtz,M. (1997) Infrared detection in a beetle. Nature, 386, 773-774.

Schütz,S., Weissbecker,B., Hummel,H.E., Apel,K.H., Schmitz,H. and Bleckmann,H. (1999) Insect antenna as a smoke detector. Nature, 398, 298-299.

Sunesson,A.L., Nilsson,C.A., Andersson,B. and Blomquist,G. (1996) Volatile metabolites produced by two fungal species cultivated on building materials. Annals of Occupational Hygiene, 40, 397-410.

Tegoni,M., Campanacci,V. and Cambillau,C. (2004) Structural aspects of sexual attraction and chemical communlication in insects. Trends in Biochemical Sciences, 29, 257-264.

Thakeow,P., Angeli,S., Weissbecker,B. and S. Schütz,S. (2008) Antennal and behavioral responses of Cis boleti to fungal odor of Trametes gibbosa. Chemical Senses, 33, 379-387.

Tjeerdsma,B.F., Boonstra,M., Pizzi,A., Tekely,P. and Militz,H. (1998) Characterisation of thermally modified wood: molecular reasons for wood performance improvement. Holz als Roh- und Werkstoff, $56,149-153$.

Weissbecker,B., Holighaus,G. and Schütz,S. (2004) Gas chromatography with mass spectrometric and electroantennographic detection: analysis of wood odorants by direct coupling of insect olfaction and mass spectrometry. Journal of Chromatography A, 1056, 209-216.

Wicher,D., Schafer,R., Bauernfeind,R., Stensmyr,M.C., Heller,R., Heinemann,S.H. and Hansson,B.S. (2008) Drosophila odorant receptors are both ligand-gated and cyclic-nucleotide-activated cation channels. Nature, 452, 1007-1U10.

Wilson, B. A., Kuehs, J., and Valentine, L. E. (2010) Guidelines for ecological burning regimes for the Gnangara groundwater system. Wilson, B. A., Kuehs, J., and Valentine, L. E. 26-38. 2010. Perth, Western Australia, Department of Environment and Conservation, Government of Western Australia. Gnangara Sustainability Strategy. 


\title{
Volatile combustion products of wood attract Acanthocnemus nigricans (Coleoptera: Acanthocnemidae)
}

Sebastian Paczkowski ${ }^{1}$, Marta Paczkowska ${ }^{1}$, Stefan Dippel ${ }^{1,2}$, Gavin Flematti $^{3}$, Stefan Schütz ${ }^{1}$

Sebastian Paczkowski (Corresponding author); (S. Paczkowski), M.Sc., Dept. of Forest Zoology and Forest Conservation, Büsgen-Institute, Georg-August-University, Göttingen, Germany, Büsgenweg 3, 37077 Göttingen, Germany; e-Mail: spaczko@gwdg.de; phone: +49-551-39-3610; fax: +49-551-3922089

Running head: Volatile combustion products of wood attract $A$. nigricans

\begin{abstract}
A. nigricans is an Australian pyrophilic beetle approaching smoldering logs for mating and oviposition. We investigated the behavior of the beetles towards combustion products of cellulose (5-methylfurfural, hydroxacetone, and karrikinolide), lignin (4-methylguaiacol, 4-ethylguaiacol, eugenol), and (Z)-3-hexen-1-ol in a custom built dynamic two arm olfactometer. The beetles were attracted by 5-methylfurfural, 4-methylguaiacol, 4-ethylguaiacol, eugenol and hydroxyacetone in different concentrations. Abundances of beetles caught in traps baited with a mixture of the most attractive volatiles showed only a trend to higher numbers. These results are discussed with respect to weather conditions, complementing infrared perception and spatio-temporal distribution of the pyrophilic beetles.
\end{abstract}

\footnotetext{
${ }^{1}$ Dept. of Forest Zoology and Forest Conservation, Büsgen-Institute, Georg-August-University, Göttingen, Germany

${ }^{2}$ Dept. of Developmental Biology, Johann-Friedrich-Blumenbach-Institute of Zoology and Anthropology, GeorgAugust-University, Göttingen, Germany

${ }^{3}$ School of Biomedical, Biomolecular and Chemical Science, The University of Western Australia, Perth, Australia
} 


\section{Introduction}

Woodland fires are important for the Australian ecosystems and many plant species have adapted or become reliant on these fires. There are specialized insect species which depend on frequent fires, as well, for example the Australian "little ash beetle" Acanthocnemus nigricans (Hope, 1845) (Coleoptera: Acanthocnemidae). This insect approaches woodland fires and can be found in the ashes close to smoldering logs where they mate and oviposit (Kreiss et al., 2007). When colonizing a place which is almost free from other animal species, the beetles avoid competition and predation. Therefore, the detection of woodland fires is very important for these animals. Investigations showed that $A$. nigricans uses a specialized infrared radiation (IR) sensory organ responding to infrared frequencies emitted by forest fires (Kreiss et al., 2005; Schmitz et al., 2002; Kreiss et al., 2007).

The ability of the olfactory sense of $A$. nigricans has not yet been investigated. In the order of insects there are many examples of odor mediated behavior. Since the discovery of odorant binding proteins (OBPs) (Vogt and Riddiford 1981) the biochemistry of olfaction in insects has been investigated intensely (Tegoni et al., 2004; Hansson 1999). It was found that the detection of volatile odorant molecules by insect antennae can be very precise. This is especially prevalent where pheromones are concerned (Pelosi and Maida 1995; Wang et al., 2010), but also in combination with other senses, for host detection (Heisswolf et al., 2007). The host detection of the pyrophilic palaearctic buprestid beetle species Melanophila acuminata is mediated by a combination of IR radiation and different methoxyphenols (Schütz et al., 1999; Schmitz et al., 1997). Methoxyphenols are the products of incomplete oxidative lignin combustion (Oasmaa 2003 et al.; Mehmetli et al., 2008).

In this study, we investigated the behavior of A.nigricans towards common products of the incomplete thermal oxidation of wood tissue. We chose hydroxyacetone, 5-methylfurfural and karrikinolide, as products of the thermal oxidation of cellulose (Piskorz et al., 1986; Zhang et al., 2008; Oasmaa et al., 2003; Flematti et al., 2011) and 4-methylguaiacol, 4-ethylguaiacol and eugenol, as common products of the thermal oxidation of lignin (Azeez 2010 et al.; Branca et al., 2006; Ingram et al., 2008; Karagoz et al., 2005). (Z)-3-hexen-1-ol, as a green leaf volatile being a host plant compound not related to fire, was also tested. For the behavioral studies we used a custom built multiple two arm olfactometer. For the field test we used flight interception traps with a volatile mixture (5-methylfurfural, 4-methylguaiacol, 4-ethylguaiacol, and eugenol).

\section{Material and Methods}

\section{Animals (collection and rearing)}

Adult $A$. nigricans were collected in two eucalypt forests shortly after wildfires in the Dwellingup State Forest, Western Australia, and in the Mundaring State Forest, Western Australia, in summer 2009 and 2010. The beetles were found in the warm ashes close to smoldering logs and stumps one to ten days after a fire. They were imported to Germany and kept in plastic containers with a $10 \%$ saccharose sugar water and water diet at $25 \pm 1{ }^{\circ} \mathrm{C}, 40 \%$ relative humidity and a $12 / 12$ day/night period. The beetles were tested for two weeks in an olfactometer, each beetle was tested on each stimulus and due to mortality the number of beetles decreased from 80 to 45 during this time. 


\section{Selected stimuli for behavioral studies}

In order to apply odor stimulation in a physiological concentration range, odorants were diluted in a solvent (paraffin oil, Uvasol ${ }^{\circledR}$, Merck) which had been shown to evoke no behavioral response. Dilution factors ranged from $10^{-2}$ to $10^{-4}(\mathrm{mg} / \mathrm{mg})$ depending on the compound. We tested the methoxyphenols 4-methylguaiacol, 4-ethylguaiacol, and eugenol, which derive from the thermal oxidation of lignin. All three compounds are medium volatile compounds (see vapor pressure in Table 1). 5-Methylfurfural was tested as a typical product of the thermal oxidation of cellulose (Zhang et al., 2008; Oasmaa et al., 2003). 5-Methylfurfural is a medium volatile compound (Table 1). We further tested hydroxyacetone as a highly volatile breakdown product of cellulose (Piskorz et al., 1986) and karrikinolide, a semi volatile breakdown product of cellulose known to facilitate sprouting of plant seeds after a fire (Flematti et al., 2005, Flematti et al., 2011). The highly volatile green leaf volatile (Z)-3-hexen-1-ol (Table 1) (Feussner et al., 1997) was also tested as a host plant volatile that is not related to fire.

Table 1 lists the compounds used in this study with information about CAS number, company from which the compound was purchased, purity of the compound, vapor pressure in $\mathrm{mm} \mathrm{Hg}$ and the dilution tested in the behavioral experiments. The values of the vapor pressure were measured under SATP (Standard Ambient Temperature and Pressure) conditions $\left(25^{\circ} \mathrm{C}\right.$ and $\left.1013 \mathrm{hPa}\right)$.

\begin{tabular}{|c|c|c|c|c|c|}
\hline Compound & CAS No. & Company & $\begin{array}{l}\text { Purity } \\
\text { [\%] }\end{array}$ & $\begin{array}{c}\text { Vapor } \\
\text { pressure } \\
{[\mathrm{mm} \mathrm{Hg}]}\end{array}$ & $\begin{array}{c}\text { Dilution testec } \\
\text { in experiment } \\
\text { in paraffin oil } \\
{[\mathrm{mg} / \mathrm{gm}]}\end{array}$ \\
\hline \multicolumn{6}{|l|}{ Lignin } \\
\hline 4-Methylguaiacol & $93-51-6$ & Aldrich & 98 & 0,078 & $10^{-2}, 10^{-4}$ \\
\hline 4-Ethylguaiacol & $2785-89-9$ & SAFC & 98 & 0,017 & $10^{-2}, 10^{-4}$ \\
\hline Eugenol & $97-53-0$ & Merck & 98 & 0,01 & $10^{-2}, 10^{-4}$ \\
\hline \multicolumn{6}{|l|}{ Cellulose } \\
\hline 5-Methylfurfural & $620-02-0$ & Across & 98 & 0,644 & $10^{-2}, 10^{-4}$ \\
\hline Hydroxyacetone & $116-09-6$ & Aldrich & 98 & 2,95 & $10^{-2}$ \\
\hline Karrikinolide & $16275-44-8$ & $\begin{array}{l}\text { Technical synthesis (Flematti } \\
\text { 2005) }\end{array}$ & 99 & Semivolatile & $10^{-2}$ \\
\hline (Z)-3-Hexen-1-ol & $928-96-1$ & Aldrich & 98 & 1,04 & $10^{-2}$ \\
\hline
\end{tabular}




\section{Olfactometer setup}

The olfactometer was described in the manuscript "The olfaction of a fire beetle leads to new concepts for early fire warning systems", Paczkowski et al., 2012 (p.101-102).

\section{Olfactometer-Experiments}

\section{Experiment $\mathbf{A}$}

Prior to the choice tests the olfactometer was tested for possible positional bias with 50 beetles, using filtered and humidified air, which was led through the walking chambers from both sides of the olfactometer. As the beetles did not prefer any of the two olfactometer arms, all stimulus compounds were tested against filtered and humidified air. In the first behavioral test the compounds 5-methylfurfural, 4-methylguaiacol, 4-ethylguaiacol, eugenol and pure paraffin oil were tested against pure air. The compounds were diluted in paraffin oil to a concentration of $10^{-2} \mathrm{mg} / \mathrm{mg}$. The position of the stimulus was changed after every run with ten beetles and the olfactometer was turned by $180^{\circ}$ after every second run with ten beetles.

\section{Experiment B}

In the second behavioral test the same compounds as in experiment $A$ were tested in dilutions of $10^{-4}$ $\mathrm{mg} / \mathrm{mg}$. Paraffin oil did not show any visible behavioral activity in experiment A. However, an attractive trend in comparison to pure air could be calculated. Therefore, we tested the odor stimulus against pure paraffin oil in this experiment. We used paraffin oil from both directions as the control and pure air from both directions as a second control.

\section{Experiment C}

In the third set of behavioral tests, hydroxyacetone, (Z)-3-hexen-1-ol, and 3-methyl-2H-furo[2,3c]pyran-2-one (karrikinolide) were tested in a dilution of $10^{-2} \mathrm{mg} / \mathrm{mg}$ against paraffin oil.

\section{Walking tracks}

The experimental setup used for these studies allows the recording of walking tracks, which display in detail the behavior of each beetle. Selected walking tracks for eugenol and paraffin oil were displayed to show typical attractive and typical random behavior. It should give a better impression of the detailed behavior of the beetles and should underline the functionality of the custom build olfactometer. The walking tracks for eugenol were among the most attractive in experiment $A$.

\section{Field Test in Australia}

Flight interception traps (type "Witaprall Ecco"; Witasek, Feldkirchen, Germany) made of black plastic boards, a black funnel and a collection container were placed randomly in two fire damaged eucalypt forest areas at Jurien Bay, Western Australia, in summer 2010 and 2011. Area one (Knights track) was a 2 ha eucalypt forest on the top of a small hill surrounded by bush land, 1 ha of the forest subject to a full fire at the north of the hill. Area two (Banovich Road) was situated at the margin of a large burned eucalypt forest. On each area we placed five traps with a volatile mixture (5methylfurfural, 4-methylguaiacol, 4-ethylguaiacol, and eugenol in arbitrarily chosen equal amounts in dilution of $10^{-2} \mathrm{mg} / \mathrm{mg}$ in paraffin oil) and five traps with pure paraffin oil. This combination and concentration of compounds was chosen as these compounds were highly attractive in the olfactometer experiment A (Paczkowski et al., 2013). The minimal distance between the traps was $5 \mathrm{~m}$ and they covered an area of approximately one hectare. 
The mass release rate of the traps with volatile mixture matches the estimated release rate of a log of ca. $0.5 \mathrm{~kg}$ smoldering during 5 days in order of magnitude (Koppmann et al., 2005; Oros and Simoneit 2001; Akagi et al., 2011). Beetles had been observed previously to take directed flight towards such small sources of smoke on burnt forest sites.

Five additional traps with the volatile mixture were placed randomly in an unburned eucalypt forest at a distance of $30 \mathrm{~m}$ from area one and five were placed randomly in a patch of unburned eucalypt trees surrounded by unburned grassland at a distance of $500 \mathrm{~m}$ from area two. Between each trap there was a minimum distance of $10 \mathrm{~m}$. The traps in the burned eucalypt forests were placed on an area of approximately one hectare. All of the traps were placed 3 to $10 \mathrm{~cm}$ above the ground in order to catch low flying insects. Such a behavior was previously observed with flying A. nigricans approaching a smoldering log. The traps were controlled daily in the afternoon, from the first to the fifth day after the fire. In this period, a weather station at Jurien Bay recorded $25 \pm 7{ }^{\circ} \mathrm{C}, 16 \pm 1 \mathrm{~km} / \mathrm{h}$ wind speed and $52 \pm 8 \%$ relative humidity.

\section{Statistical Analysis}

\section{Olfactometer experiments}

The program Statistica 7.1 (StatSoft Inc., USA) was used for statistical analysis. The behavior of each beetle was expressed by an individual attractivity index, which was the mean value of the recorded positions. The attractivity index was calculated, expressing the mean value of the beetle position as the percentage of the theoretical maximum value, which would be 500 in case of direct orientation towards the stimulus. In this case, the individual attractivity index would be $100 \%$. In case of a maximum repellent behavior the individual attractivity index would be $0 \%$. The overall attractivity index for each stimulus was calculated as the median of all individual attractivity indices, because the individual attractivity indices were not normally distributed (Shapiro Wilks Test; $p=0,05$ ). The differences between the orientation towards the tested compounds and paraffin oil were tested with the Mann-Whitney U-Test or the sign test. For experiment A we used the Mann-Whitney U-Test test, because the orientation towards a volatile compound and towards paraffin oil was tested in separate behavior tests. For the analysis of the test with humidified air from both sides of the olfactometer we used the sign test for paired samples. For experiments $B$ and $C$ we always used the sign test for paired samples, because each beetle was tested against the internal paraffin oil control. For each test, we used the positions of the beetles outside the neutral zone (Paczkowski et al., 2011), which eliminated weak beetles that did not or almost not move. For both tests significance was rejected when the $p$-Level was higher than $5 \%$. P-Levels below $5 \%$ were indicated by one star, below $0.5 \%$ by two stars and below $0.1 \%$ by three stars.

\section{Field test}

Abundances of beetles caught on the four different sites by volatile baited traps and control traps were evaluated statistically by a $\chi^{2}$ test.

\section{Results \\ Choice tests}

The control test with air resulted in a median of $49 \%$ for the overall attractivity index (Figure 1 ). The paraffin oil control showed a median of $54 \%$, not significantly different from the air control. In 
experiment $A$, all of the tested compounds showed an attractivity index higher than $70 \%$ being significantly different to the paraffin oil control. However, the upper quartile of paraffin oil was higher than expected for a behavior without preferences (Figure 1). This led to the decision to test the same compounds in a higher dilution directly against paraffin oil to evaluate the results of experiment $A$. In experiment $B$ and $C$ the choice test with paraffin oil from both directions showed no significance, as well (Figure 2). 5-Methylfurfural, 4-ethylguaiacol, and 4-methylguaiacol were still significantly attractive for $A$. nigricans in a dilution of $10^{-4} \mathrm{mg} / \mathrm{mg}$. All compounds had an attractivity index of 72 or $73 \%$. Eugenol was not significantly attractive with an attractivity index of $56 \%$ (Figure 2). The attractivity index of hydroxyacetone (72\%) is in the same range as that of 5-methylfurfural, 4methylguaiacol or 4-ethylguaiacol in concentrations of $10^{-4} \mathrm{mg} / \mathrm{mg}$. (Z)-3-hexen-1-ol and 3-methyl$2 \mathrm{H}$-furo[2,3-c]pyran-2-one do not appeal to A. nigricans (Figure 3).

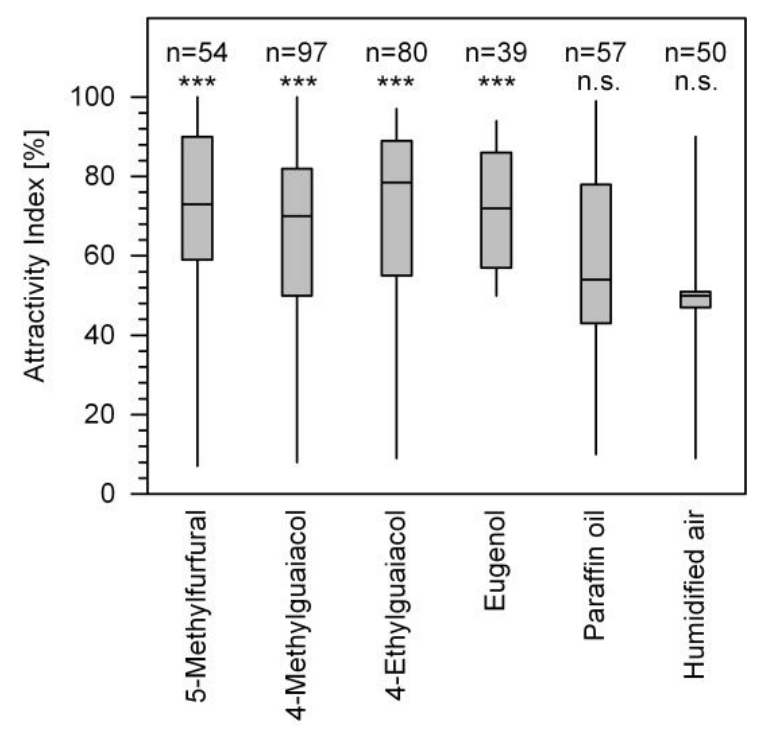

Figure 1 shows the attractivity indexes for experiment A. Bars and whiskers display the median, upper and lower quartiles and maxima and minima. All compounds were tested in a concentration of $10^{-2} \mathrm{mg} / \mathrm{mg}$ against humidified air. The $n$-value shows the number of insects used for each choice test. The Mann Whittney U-test was used to calculate significant differences between the responses to the paraffin oil control and the stimuli (n.s. = not significant; ${ }^{*}, p<0.05$; $* *, p<0.005 ; * * *, p<0.001)$. 


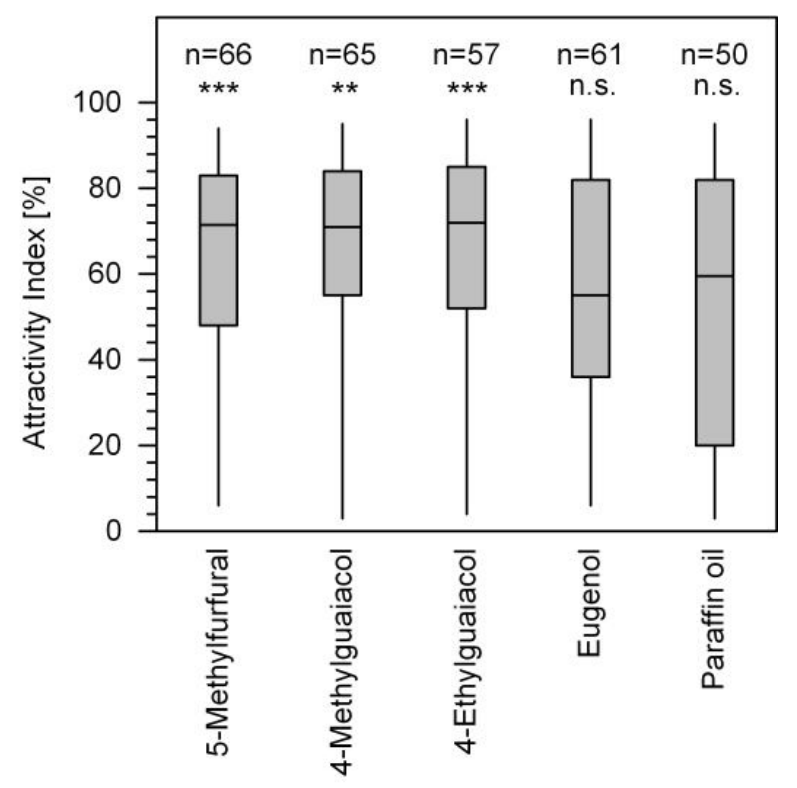

Figure 2 shows the attractivetly indexes for experiment B. Bars and whiskers display the median, upper and lower quartiles and maxima and minima. All compounds were tested in a concentration of $10^{-4} \mathrm{mg} / \mathrm{mg}$ against paraffin oil. The $\mathrm{n}$-value shows the number of insects used for each choice test. The sign-test was used to calculate significant differences between the responses to the internal paraffin oil control and the stimulus (n.s. $=$ not significant; ${ }^{*}=p<0.05 ;{ }^{* *}=p<0.005$; $* * *=p<0.001)$.

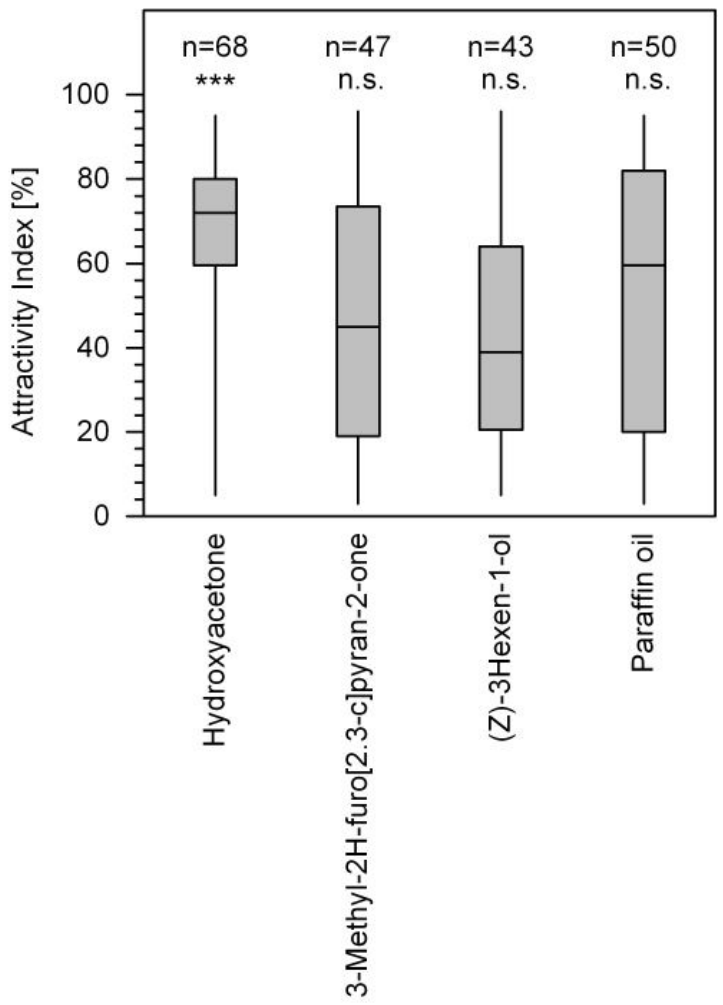

Figure 3 shows the attractivity indexes for experiment C. Bars and whiskers display the median, upper and lower quartiles and maxima and minima. All compounds were tested in a concentration of $10-2 \mathrm{mg} / \mathrm{mg}$ against paraffin oil. The $\mathrm{n}$-value shows the number of insects used for each choice test. The sign-test was used to calculate significant differences between the responses to the internal paraffin oil control and the stimulus (n.s. $=$ not significant; ${ }^{*}=p<0.05 ;{ }^{* *}=p<0.005$; $* * *=p<0.001)$. 


\section{Walking tracks}

In Figure 4, the walking tracks of ten selected beetles for eugenol in a concentration of $10^{-2} \mathrm{mg} / \mathrm{mg}$ (experiment $A$ ) and paraffin oil (experiment $A$ ) are displayed (Figure 4). On the $y$-axis the position 0 is the side of the olfactometer without stimulus compound and the posititon 500 is the side with the stimulus compound. The $\mathrm{x}$-axis begins with second one, as it took one second to remove the comb and release the beetles. The recorded starting point of the beetles is not 250 , because the first choice of the beetles is shown in the first second. Almost all of the beetles were quite active and most of the beetles directly oriented towards eugenol (Figure 4). The beetles which chose the other arm changed their direction and went to the stimulus. When exposed to paraffin oil, the beetles did not show the same first choice behavior. Some beetles walked through the olfactometer without showing preference and others remained on the side of their first choice. However, paraffin oil is slightly attractive (Figure 1).
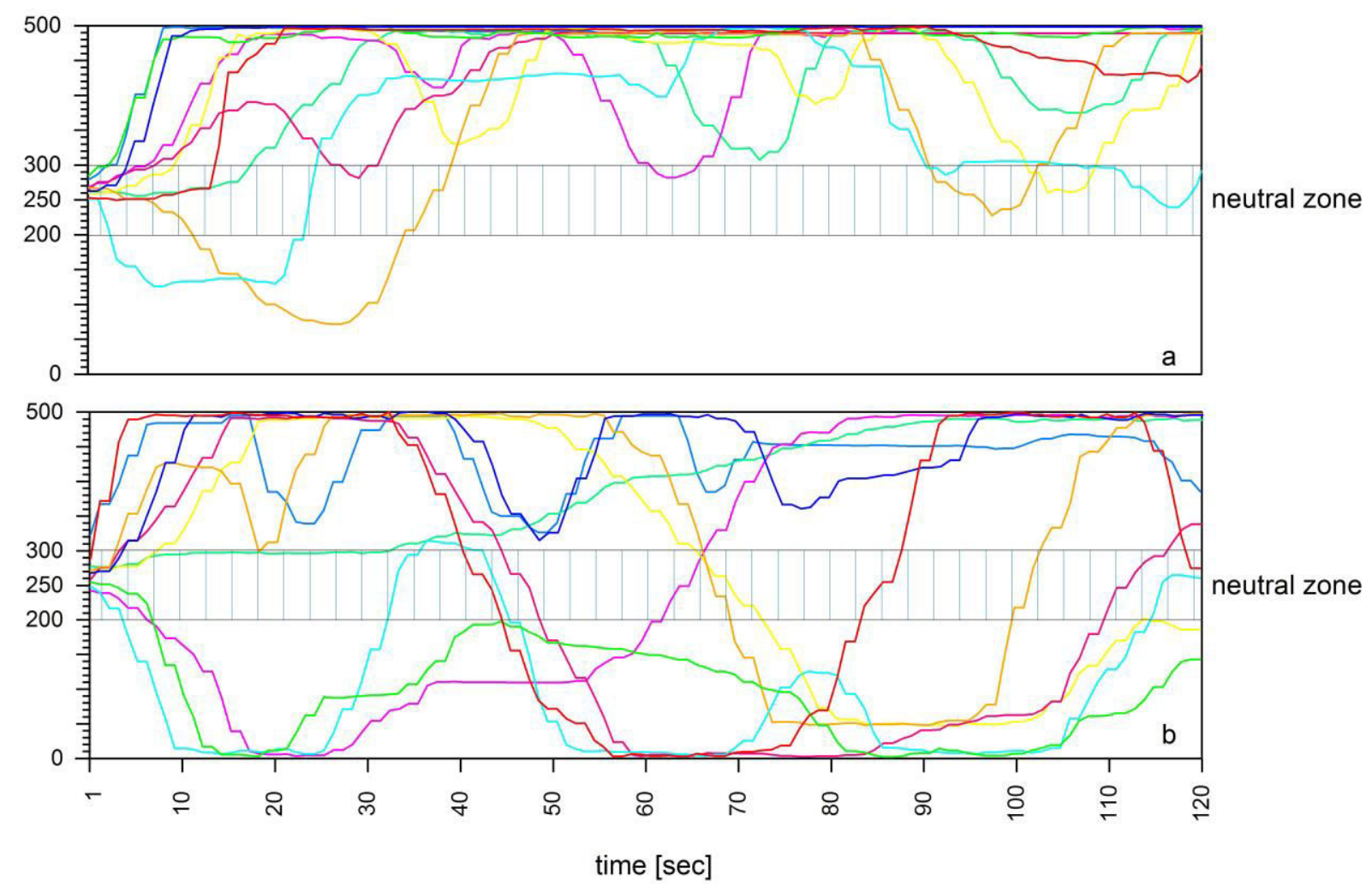

Figure 4 shows the tracks of ten beetles in the time period of two minutes for (a) eugenol in dilution of $10-2 \mathrm{mg} / \mathrm{mg}$ in paraffin oil against air and (b) pure paraffin oil against air in experiment $A$. The walking tracks of the beetles when exposed to eugenol in concentration of $\mathbf{1 0 - 2} \mathrm{mg} / \mathrm{mg}$ in paraffin oil are an example for the behavior towards a strongly attractive compound. The walking tracks when exposed to paraffin oil are an example for the behavior towards an unattractive compound.

\section{Field test in Australia}

During the field test, $35 \mathrm{~A}$. nigricans were caught in the flight interception traps. 20 beetles $(57 \%)$ were caught in the traps with the volatile mixture and 15 beetles $(43 \%)$ were caught in the traps with paraffin oil. On the first area, eleven beetles (61\%) were caught in the traps with the volatile mixture and seven (39\%) in the traps with paraffin oil. On the second area, nine beetles (53\%) were caught in the traps with the volatile mixture and eight $(47 \%)$ were caught in the traps with paraffin oil. The abundance of beetles caught in volatile baited traps and control traps did not show significant differences. On the unburned forest areas no beetles were caught (Figure 5). The abundance of 


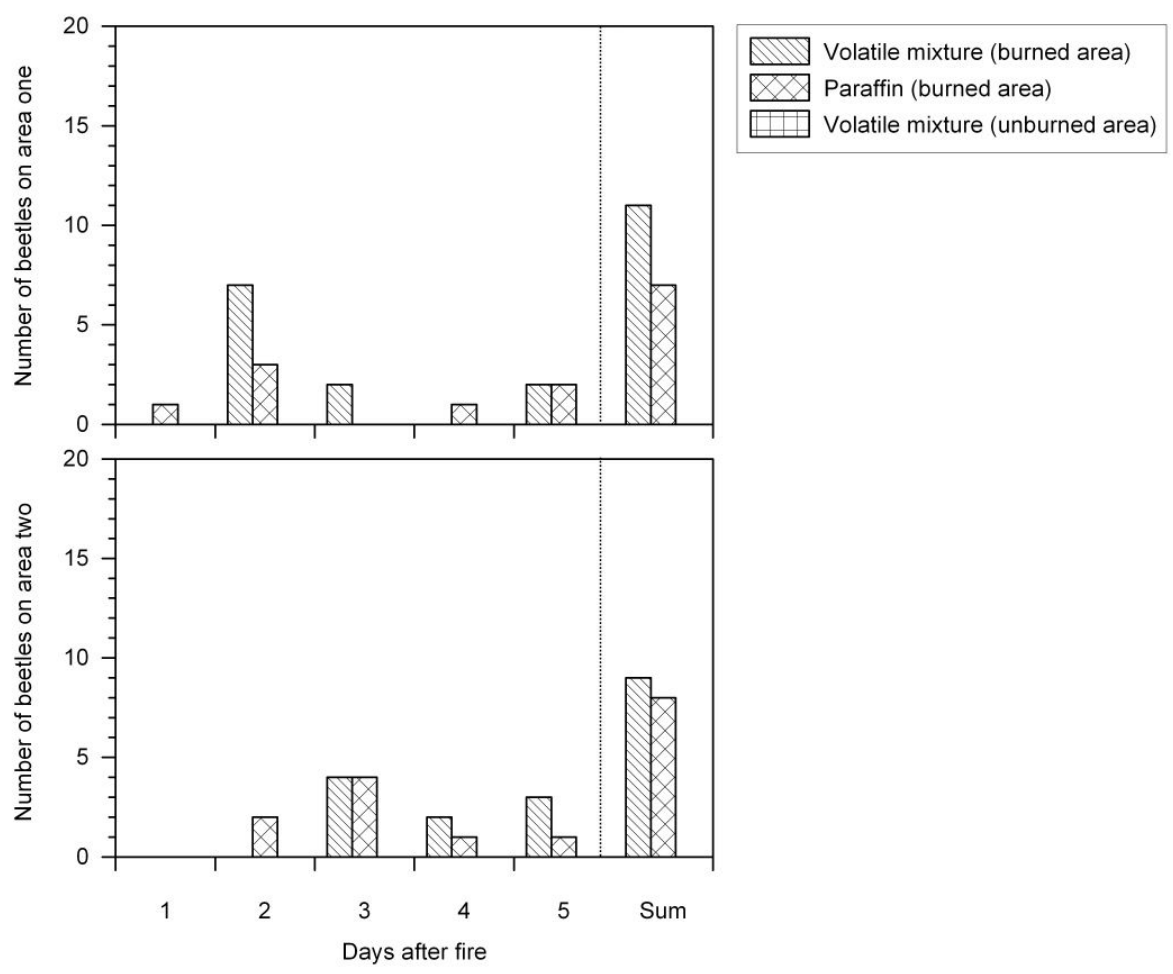

Figure 5 shows the number of beetles caught during the field test for both areas on each day. The sum shows the overall number of $\boldsymbol{A}$. nigricans caught in the traps. On both unburned areas no beetles were caught.

beetles caught on burned sites compared to those caught on unburned sites did show highly significant differences (国2 test, $\mathrm{P}<0.001$ ).

\section{Discussion}

According to Martel and Boivin (Martel and Boivin, 2011), our first experiment A tests the apparent choice of the beetles. An apparent choice experiment tests a perceived stimulus against a stimulus that is not perceived. This does not prove the orientation towards the stimulus in a choice situation, because the insects might orientate to any stimulus because it is the only one. In this experiment $(A)$ the stimulus was tested against pure air, which is not a perceived odor. Therefore, the third requirement introduced by Martel and Boivin, which requires the test against a perceived stimulus, is not fulfilled. Of course that shows that the beetles are able to detect this source, but the results of experiment $A$ do not show that the beetles prefer the tested combustion products of cellulose and lignin in a choice situation. Therefore, experiment A does not prove that the beetles would orientate towards smoldering logs in a natural environment. However, the high attractivity index of combustion products in comparison to the slight attraction of paraffin oil in experiment $A$ indicates a higher importance of the combustion products. This tendency is shown in experiment $B$, as well, where the apparent choice is tested, because it is not known if the beetles perceive paraffin oil. However, the results of experiment $B$ indicate a high importance of 5-methylfurfural, 4methylguaiacol and 4-ethylguaiacol for A. nigricans (Figure 2), because these compounds are attractive even in a higher dilution. Although the attractivity index of eugenol was the highest in experiment $A$ and most of the beetles walked directly towards the compound and stayed there (Figure 4), it is much lower at a concentration of $10^{-4} \mathrm{mg} / \mathrm{mg}$. It is possible that eugenol is a close range volatile (Walter et al., 2010), which is used by the beetles to identify and select places for 
oviposition when they arrive on a freshly burned area. They may respond to the volatile compound in high concentration because this resembles the concentration of smoldering logs. In experiment $\mathrm{C}$ the attractivity index of hydroxyacetone is in the same range as that of 5-methylfurfural, 4methylguaiacol and 4-ethylguaiacol. However, it is not possible to compare the behavior of the beetles towards these three compounds directly, as they have different vapor pressures (Table 1). This results in different concentrations of the tested compounds in the airflow. Hydroxyacetone is more attractive than (Z)-3-hexen-1-ol, which has a comparable vapor pressure (Table 1). Karrikinolide (3-methyl-2H-furo[2,3-c]pyran-2-one) is a compound with a very low vapour pressure and might be a short range volatile compound, which could be detectable for the beetles when they are close to smoldering logs (Walter et al., 2010).

The traps baited with the volatile mixture showed only slightly increased numbers of beetle catches in comparison to traps with paraffin oil on both areas. Moreover, the overall number of beetles caught was very low. However, it blew a steady wind with a speed of about $16 \mathrm{~km} / \mathrm{h}(4.4 \mathrm{~m} / \mathrm{s})$ during the whole experiment. Air velocities of that magnitude compromise the ability of oriented upwind flight by these small beetles as indicated by the high number of catches in control (paraffin oil only) traps because of physical flight interception. Thus, the higher numbers of beetles observed in traps baited with the volatile mixture might be regarded as some support of the olfactometer-results which were obtained under more favorable conditions (linear air velocity: $0.01 \mathrm{~m} / \mathrm{s}$ ). Beetles were caught in the traps with the volatile mixture neither on the unburned area near to the burned area, nor on the unburned area in larger distance to the burned area. Probably the high wind speed diluted the volatiles released from the trap making oriented upwind flight towards such a small source even more difficult for the beetles. Moreover, adult A. nigricans were depleted from the unburned areas because they had been attracted to the burned area still providing some bigger smoldering logs. Considering that the tested compounds are emitted by smoldering logs (Azeez et al., 2010; Branca et al., 2006; Ingram et al., 2008; Karagoz et al., 2005; Zhang et al., 2008; Oasmaa et al., 2003), the beetle is known to be attracted by smoldering logs in its natural environment (Kreiss et al., 2007), and the compounds were attractive in olfactometer-tests, it seems likely that another stimulus besides the olfactory stimulus like infrared radiation is needed by the beetle to complete the behavioral program of approaching its mating and oviposition site (Heisswolf et al., 2007; Kreiss et al., 2005; Schmitz et al., 2002; Kreiss et al., 2007).

An alternative interpretation of the strong concentration of beetles to the burnt site could be that the combination of heat from flames and the presence of combustion volatiles might induce the hatching of the beetles, in analogy to the mechanisms found in plants or fungi (Wilson et al., 2010, Flematti et al., 2004) and that olfactory and infrared stimuli only serve in short range orientation keeping the beetles on the burned site (Evans 2010). This would need the existence of a prolonged diapause to survive the time between two fire events. Such prolonged diapauses usually exist for insect species living in habitats that are unpredictable, such as deserts or the arctic, but they can also occur in ordinary environments (Danks 2006). Fires in Australia could be such an unpredictability, and the estimated duration of the diapause might be in the range of 3-5 years according to the discussion on aboriginal burnings (Burrows 2008) and 10 to 15 years according to the average time an area burned in the Gnangara Groundwater System from 1970 to 2009 (Wilson et al., 2010). The existence of such a diapause would explain the immediate presence of the beetles after a fire, although their ability in flying is restricted and not comparable to other pyrophilic species like $M$. atrata or Melanophila species (Evans 2010). 


\section{Conclusion}

Our results show that $A$. nigricans is attracted by volatile combustion products of cellulose and lignin in a dynamic two-arm olfactometer. The field studies indicate that the olfactory sense of the beetle might complement its IR perception and that its capability to reach forest fire sites might depend on weather conditions.

Further research should concentrate on measurements of release rates and distance-dependent concentrations of the stimulus compounds from forest fires and artificial volatile sources and on combined experiments using improved volatile baited traps, infrared-sources and a combination of both. Moreover, the assessment of fire frequencies (Wilson et al., 2010, Wittkuhn et al., 2011) and the assessment of beetle densities as affected by fire history of the sites will be required. This can lead to more realistic distance estimations for fire detection in pyrophilic insects (Evans 2010) and can contribute to an increased understanding of spatio-temporal distribution pattern of pyrophilic insects.

\section{Acknowledgements}

We like to thank Prof. Barbara Wilson, Prof. Helmut Schmitz, Dr. Neil Burrows, Mike Cantelo, Brian Inglis and Jan Seelig for scientific and technical support during our presence in Western Australia. We like to thank the fire fighters of Western Australia and the staff of the Department of Environment and Conservation, Western Australia, for supporting our research. The work of Stefan Dippel was kindly supported by DFG Priority Programme/ SPP 1392: Integrative Analysis of Olfaction. The work of Marta Paczkowska was kindly supported by the BMBF-Bundesministerium für Bildung und Forschung. The work of Sebastian Paczkowski was kindly supported by the Cusanuswerk, Bischöfliche Studienförderung, Germany.

\section{References}

Akagi,S.K., Yokelson,R.J., Wiedinmyr,C., Alvarado,M.J., Reid,J.S., Karl,T., Crounse,J.D., Wenneberg,P.O. (2011) Emission factors for open and domestic biomass burning for use in atmospheric models. Atmospheric Chemistry and Physics, 11, 4039-4072.

Azeez,A.M., Meier,D., Odermatt,J. and Willner,T. (2010) Fast pyrolysis of african and european lignocellulosic biomasses using Py-GC/MS and fluidized bed reactor. Energy \& Fuels, 24, 2078-2085.

Branca,C., Di Blasi,C. and Elefante,R. (2006) Devolatilization of conventional pyrolysis oils generated from biomass and cellulose. Energy \& Fuels, 20, 2253-2261.

Burrows,N.D. (2008) Linking fire ecology and fire management in south-west Australian forest landscapes. Forest Ecology Management, 255, 2394-2406.

Danks,H.V. (2006) Key themes in the study of seasonal adaptations in insects II. Life-cycle patterns. Applied Entomology and Zoology, 41, 1-13.

Evans,W.G. (2010) Reproductive role of infrared radiation sensors of Melanophila acuminata (Coleoptera: Buprestidae) at forest fires. Annuals of the Entomological Society of America 103, 823826. 
Feussner,I., Kühn,H. and Wasternack,C. (1997) Do specific linoleate 13-lipoxygenases initiate $\beta$ oxidation? FEBS Letters, 406, 1-5.

Flematti, G.R., Ghisalberti, E.L., Dixon, K.W., Trengove, R.D. (2004) A compound from smoke that promotes seed germination. Science 305, 977-977.

Flematti, G.R., Ghisalberti, E.L., Dixon, K.W., Trengove, R.D. (2005) Synthesis of the seed germination stimulant 3-methyl-2H-furo[2,3-c]pyran-2-one. Tetrahedron Letters, 46, 5719-5721.

Flematti,G.R., Scaffidi,A., Dixon,K.W., Smith,S.M. and Ghisalberti,E.L. (2011) Production of the seed germination stimulant karrikinolide from combustion of simple carbohydrates. Journal of Agricultural and Food Chemistry, 59, 1195-1198.

Hansson, B.S. (1999) Insect olfaction. Springer, New York, USA.

Heisswolf,A., Gabler,D., Obermaier,E. and Muller,C. (2007) Olfactory versus contact cues in host plant recognition of a monophagous chrysomelid beetle. Journal of Insect Behavior, 20, 247-266.

Ingram,L., Mohan,D., Bricka,M., Steele,P., Strobel,D., Crocker,D., Mitchell,B., Mohammad,J., Cantrell,K. and Pittman,C.U. (2008) Pyrolysis of wood and bark in an auger reactor: Physical properties and chemical analysis of the produced bio-oils. Energy \& Fuels, 22, 614-625.

Karagoz,S., Bhaskar,T., Muto,A. and Sakata,Y. (2005) Comparative studies of oil compositions produced from sawdust, rice husk, lignin and cellulose by hydrothermal treatment. Fuel, 84, 875-884.

Koppmann,R., Von Czapiewski,K., Reid,J.S. (2005) A review of biomass burning emissions, part $i$ : gaseous emissions of carbon monoxide, methane, volatile organic compounds, and nitrogen containing compounds. Atmospheric Chemistry and Physics Discussion, 5,10455-10516.

Kreiss,E.J., Schmitz,A. and Schmitz,H. (2005) Morphology of the prothoracic discs and associated sensilla of Acanthocnemus nigricans (Coleoptera, Acanthocnemidae). Arthropod Structure \& Development, 34, 419-428.

Kreiss,E., Schmitz,H. and Gebhardt,M. (2007) Electrophysiological characterisation of the infrared organ of the Australian "Little ash beetle" Acanthocnemus nigricans (Coleoptera, Acanthocnemidae). Journal of Comparative Physiology A-Neuroethology Sensory Neural and Behavioral Physiology, 193, 729-739.

Martel,V. and Boivin,G. (2011) Do choice tests really test choice?. Journal of Insect Behavior, DOI 10.1007/s10905-011-9257-9.

Mehmetli,E., Dogan,O., Tiris,M., Kiran,N.C. and Matuschek,G. (2008) Thermolysis product distribution of solid waste obtained from olive oil production. Clean-Soil Air Water, 36, 315-319.

Oasmaa,A., Kuoppala,E. and Solantausta,Y. (2003) Fast pyrolysis of forestry residue. 2. Physicochemical composition of product liquid. Energy \& Fuels, 17, 433-443.

Oros,D.R., Simoneit,B.R.T. (2001) Identification and emission factors of molecular tracers in organic aerosols from biomass burning part 2. Deciduous trees. Applied Geochemistry, 16,1545-1565.

Paczkowski,S., Paczkowska,M., Dippel,S., Schulze,N., Schütz,S., Sauerwald,T., Weiss,A., Bauer,M., Gottschald,J. and Kohl,C.-D. (2013) The olfaction of a fire beetle leads to new concepts for early fire warning systems. Sensors and Actuators. In peer-revision, submitted 28.10.2012. 
Pelosi,P. and Maida,R. (1995) Odorant-binding proteins in insects. Comparative Biochemistry and Physiology B-Biochemistry \& Molecular Biology, 111, 503-514.

Piskorz,J., Radlein,D. and Scott,D.S. (1986) On the mechanism of the rapid pyrolysis of cellulose. Journal of Analytical and Applied Pyrolysis, 9, 121-137.

Schmitz,H., Bleckmann,H. and Murtz,M. (1997) Infrared detection in a beetle. Nature, 386, 773-774.

Schmitz,H., Schmitz,A., Trenner,S. and Bleckmann,H. (2002) A new type of insect infrared organ of low thermal mass. Naturwissenschaften, 89, 226-229.

Schütz,S., Weissbecker,B., Hummel,H.E., Apel,K.H., Schmitz,H. and Bleckmann,H. (1999) Insect antenna as a smoke detector. Nature, 398, 298-299.

Tegoni,M., Campanacci,V. and Cambillau,C. (2004) Structural aspects of sexual attraction and chemical communlication in insects. Trends in Biochemical Sciences, 29, 257-264.

Vogt,R.G. and Riddiford,L.M. (1981) Pheromone binding and inactivation by moth antennae. Nature, 293, 161-163.

Walter,A.J., Venette,R.C., Kells,S.A. and Seybold,S.J. (2010) Walking response of the mediterranean pine engraver, Orthotomicus erosus, to novel plant odors in a laboratory olfactometer. Journal of Insect Behavior, 23, 251-267.

Wang,P., Lyman,R.F., Mackay,T.F.C. and Anholt,R.R.H. (2010) Natural variation in odorant recognition among odorant-binding proteins in Drosophila melanogaster. Genetics, 184, 759-767.

Wilson, B. A., Kuehs, J., and Valentine, L. E. (2010) Guidelines for ecological burning regimes for the Gnangara groundwater system. Wilson, B. A., Kuehs, J., and Valentine, L. E. 26-38. 2010. Perth, Western Australia, Department of Environment and Conservation, Government of Western Australia. Gnangara Sustainability Strategy.

Wittkuhn,R.S., McCaw,L., Wills,A.J., Robinson,R., Andersen,A.N., Van Heurck,P., Farr,J., Liddelow,G., Cranfield,R. (2011) Variation in fire interval sequences has minimal effects on species richness and composition in fire-prone landscapes of south-west Western Australia. Forest Ecology and Management 261:965-978.

Zhang,B., Huang,H.J. and Ramaswamy,S. (2008) Reaction kinetics of the hydrothermal treatment of lignin. Applied Biochemistry and Biotechnology, 147, 119-131. 


\title{
Biomimetic gas sensors for large-scale drying of wood particles
}

\author{
Sebastian Paczkowski ${ }^{a}$, Tilman Sauerwald ${ }^{b}$, Alexander Wei $\beta^{b}$, Marco Bauer $^{b}$, Dieter Kohl ${ }^{b}$, Stefan \\ Schütz*a \\ ${ }^{a}$ Forest Zoology \& Forest Protection; University Göttingen, Büsgenweg 3, 37077 Göttingen, Germany \\ ${ }^{\mathrm{b}}$ Institute of Applied Physics, University Giessen, Heinrich-Buff-Ring 16, 35392 Giessen, Germany
}

\begin{abstract}
The sensitivity and selectivity of insect antennae are evolutionary tuned to specific needs of the insect. The Australian pyrophilic beetle Merimna atrata needs freshly heated wood to bring up its offspring and, consequently, shows a very high sensitivity to volatiles specific for wood-fires and heated wood. Volatile organic compounds released by wood particles heated at different temperatures were collected. Parallel trace analytical examination and antennal responses of the pyrophilic beetles to volatiles released by the wood reveal a highly differentiated detection system of these insects for early and late products of wood fires. This enabled a selection of marker compounds used by insects since several million years for the discrimination of different stages of wood fires. In the industrial production of engineered wood such as particle boards, wooden particles are dried in large-scale high temperature dryers. Air temperatures between $150-600^{\circ} \mathrm{C}$ are essential for the required material flow in the particle board production. Despite the resulting energy-efficiency of high temperature drying, high temperatures are avoided because of the increased risk of spontaneous combustion. Losses in productivity caused by fire have a strong impact on the whole production system. In order to raise the drying temperature without risking a fire, it is important to develop a monitoring system that will reliably detect early fire stages by their characteristic volatile pattern. Thus, perception filters and evaluation algorithms of pyrophilic insects can provide blue prints for biomimetic gas sensors for large-scale drying of wood particles. Especially tungsten oxide sensor elements exhibit a high sensitivity to some of the key substances. Their high sensitivity and selectivity to terpenes and aldehydes in combination with high sensitivity and selectivity of tin oxide sensor elements to hydroxylated and phenolic compounds, both showing low cross-reactivity with water and carbon monoxide, mimic highly efficient biological fire detection systems.
\end{abstract}

Keywords: Insect, olfaction, pyrophilic, wood fire, particle board, tungsten oxide, nanocasting

\section{Introduction}

\section{Pyrophilic beetles}

Already in the beginning of the $20^{\text {th }}$ century firemen reported the presence of black beetles that flew in masses towards burning forests, disturbing the fire extinguishing and even attacked the firemen. In the reports about the unusual pyrophilic behavior of the black jewel beetle Melanophila 
acuminata speculations arose that the beetle might be able to orientate towards a forest fire by means of its olfaction.

These fire loving (pyrophilous) beetles depend on forest fires for their reproduction. Such insects approach ongoing fires and invade the burnt area immediately after fire. For long-range orientation toward a fire as well as for the short-range orientation on a freshly burnt area these insects have special sensors for smoke (Schütz et al., 1999a) and infrared radiation (Schmitz et al 1997). Whereas the olfactory receptors for smoke are located on the antennae, the infrared receptors are housed in extra-antennal sensory organs, which can be found on the thorax or on the abdomen. Two genera of jewel beetles (Buprestidae) can be classified as pyrophilous: More than ten species of the genus Melanophila, which are distributed nearly all over the northern hemisphere, and the buprestid beetle Merimna atrata, which is endemic to Australia (Schmitz and Schmitz 2002).

Despite the fact that Melanophila and Merimna show nearly the same behaviour and belong to the same family of jewel beetles, their infrared receptors are totally different from each other, suggesting that this specialization to fire developed independently in both genera from the northern hemisphere and from Australia.

Once on the freshly burnt area, the males of both genera often stay close to burning or smoldering wood looking for conspecific females. After mating, the females deposit eggs under the burnt bark of the trees. Only there, the wood-boring larvae of Melanophila and Merimna can develop by feeding in the weakened and pre-heated wood of fire damaged trees (Apel 1989). Consequently fire detection is the compulsory precondition for the survival of all these pyrophilous insect species. They should be able to detect fires from distances as large as possible. Moreover, they have to be able to discriminate trees that are charred to the core from trees that still contain pre-heated, but uncharred wood as substrate for their larvae.

Progressing research on Melanophila acuminata revealed, that its antenna detects smoke components such as guajacol compounds very sensitively (Schütz et al., 1999a). These compounds originate from the thermal oxidation of lignin, one of the basic constituents of wood. As the beetles breed in burned wood, they are adapted to the detection of wood fires like forest fires. Combustion of fossil wood (i.e., coal) can be detected by the beetles, too, as it also contains lignin.

Combustion products of diesel fuel or gasoline do not elicit comparable signals. This enables the design of a biosensor based on the antenna of Melanophila acuminata to differentiate between frequently occurring background compounds, e.g. originating in traffic activity, and wood fire. Thus, false alarms caused by engine exhausts can be avoided and a reliable fire early warning can be based on the performance of the biosensor. Field experiments revealed that a fire comprising only several trees (five $\mathrm{m}^{3}$ wood) can be detected by a biosensor based on the antenna of Melanophila acuminata in two km distance and can be traced by the concentration gradient of smoke components and wind direction through a dense forest stand. Combining the high sensitivity and selectivity for marker compounds of fires with the identification of the kind of fire and the traceability of the source, a biosensor based on the antenna of Melanophila acuminata could be a strong tool for the first link in the chain of firefighting.

Once reaching the freshly burned area the female beetles lay their eggs on stems that were not completely destroyed by the fire. The hatching larvae feed on the cambium and later on the preheated wood tissue. To be able to find an appropriate place for their eggs, the beetle has to be able to distinguish between different stages of heated or burned wood tissue. This ability can result in a 
marker compound pattern that significantly correlates the emission of compounds during wood heating with the temperature of the wood.

\section{Large-scale drying of wood particles}

In the industrial production of engineered wood such as particle boards, wooden particles are dried in large-scale high temperature dryers operated at air temperatures between $150-600^{\circ} \mathrm{C}$. These high temperatures are essential for the required material flow in the particle board production, because higher temperatures accelerate the process of wood drying significantly. Despite the resulting energy-efficiency of high temperature drying, high temperatures are avoided because of the increased risk of spontaneous combustion of wood particles. Losses in productivity caused by fire have a strong impact on the production process and decrease the profit margin. Therefore, in order to raise the drying temperature without risking a fire, it is important to increase the reliability of early fire warning.

During the drying process, the heated wood particles reach average temperatures of about $150^{\circ} \mathrm{C}$. In this temperature range self-ignition of wood is possible. Heated wood emits a broad range of volatile organic compounds (VOCs). Early fire warning is possible by detection of these volatiles. However, marker volatiles have to be selected, which enable reliable identification of different stages of wood prior to and after ignition, like open fire, smoldering fire or heated wood. This is a challenging task because of the multitude and high variability of the volatile compounds released by heated or burning wood. The selection of reliable marker volatiles might be simplified by using the olfactory profile of a pyrophilic beetle. These beetles have survived on these marker compounds for several million years.

\section{Biomimetic sensors}

Analyzing odors emitted due to the metabolic activity of organisms adapted to an ecological context usually results in a high qualitative and quantitative variation of the odor constituting compounds. Despite this high variation insects are able to orientate towards the aimed source of the scents by means of their olfaction. Taking in account the recent knowledge of the biochemical chain of odor compound detection this implies that insects selectively detect marker compounds from the whole scent emitted from the desired source, despite variation factors, like environmental conditions (e.g., temperature) or metabolic activity of other organisms (e.g., microorganisms). Such marker compounds can represent distinct conditions of dynamic ecological processes.

Although nature delivers the approach towards the solution in such cases, e.g. early fire detection utilizing the odor processing of Melanophila acuminata, an adaptation to the requirements of technical processes has to be performed.

Considering the performance of biosensors on the basis of insect antennae it is not possible yet to mimic its high sensitivity and selectivity in detecting single compounds by an artificial solely technical device. Anyway, the progress in gas sensor applications provides devices with certain advantages in comparison to biosensors. The major drawback of biosensors is the short life-time of the antenna that restricts its efficient usage. Although the requirements of running a biosensor are not high, personnel for the systems operation has to be preoccupied. Thus, biosensors are more useful for onsite screening or tracking purposes than for long term monitoring purposes. In contrast, solid state sensors like semiconductor gas sensors have a lifetime of approximately one year. That makes them good candidates for low-cost monitoring applications in industrial processes. As they do not possess the selectivity and sensitivity of biosensors, their applicability is restricted concerning the 
concentration of the compounds to be traced and the level of their identity, which are rather compound groups, like aldehydes or amines than single compounds. Applications of semiconductor gas sensors are, for instance, monitoring the shelf-life of fish (Xavier 2003) of wheat for fungal contamination (Presicce 2006), detecting different mold species in buildings (Kuske 2006) and smoldering fires in coal storage units (Schütz et al., 1999a). To combine this technique with the concept of marker compounds is challenging, as the sensor has to be modified to respond sensitively and to gate out other reactions to interfering compounds. This can be achieved by semiconductor gas sensor arrays where the combined reactions of all sensors in the array lead to a compound specific pattern. In the present paper we focus on the identification of marker compounds appropriate to assess the transition from heated wood to beginning smoldering, and the characterization of solid-state sensors for the selective detection of these marker compounds in an array.

\section{Material and Methods}

\section{Sampling}

Samples of volatiles released by heated, smoldering and burning wood were obtained from different biomass fuels from German forest (beech, scots pine, grass) and Australian woodland (Eucalyptus-, Banksia-, and Melaleuca- species) carried out in laboratory, and by sampling smoke of forest fire in different stages in Germany and Australia. For scots pine, laboratory experiments were performed to investigate the differences of the volatile pattern from room temperature to burning. Two grams of pine flakes were put in a heating chamber and volatiles were sampled for $30 \mathrm{sec}$ at $25^{\circ} \mathrm{C}, 50^{\circ} \mathrm{C}, 75^{\circ} \mathrm{C}$, $100^{\circ} \mathrm{C}, 150^{\circ} \mathrm{C}, 200^{\circ} \mathrm{C}, 250^{\circ} \mathrm{C}$ and $300^{\circ} \mathrm{C}$. During the experiment, synthetic air was provided into the heating chamber in order to implement oxidative conditions. After each sampling step the temperature was raised to the next level and the flakes were conditioned for $7 \mathrm{~min}$ at the new temperature. The experiment was repeated five times. Pyrophilic beetles were caught at the forest fires in Germany and Australia and were reared in the laboratory until electrophysiological experiments were performed.

\section{Trace analysis and electroantennography}

Insect antennae were excised and mounted in a special antenna holder providing contact to a pair of $\mathrm{Ag} / \mathrm{AgCl}$-electrodes via an electrolyte solution adapted to the insect hemolymph (Schütz et al., 1999b). The antenna holder provides a stable support for the antenna in which its surface is freely accessible to the air flow from the gas-chromatograph with parallel mass-spectrometric and electroantennographic detection (GC-MS/EAG).

Calibration of antennae is obtained by puffs from a glass syringe containing an oil-dilution of the stimulus compound on filter paper in a PTFE cartouche (Schütz et al., 1999b). Standard solutions used for calibration of the GC-MS/EAG system are listed in Table 1. One microliter (equivalent to 10 ng) of each prepared volatile sample was injected splitless into a GC/MS system (Agilent GC 6890N, $5973 \mathrm{~N}$ quadrupole mass spectrometer) coupled to an adapted electroantennographic chamber. System and settings are described in (Weißbecker et al., 2004). Helium was used as carrier gas at a flow rate of $1 \mathrm{ml} \mathrm{min}{ }^{-1}$. The effluent flow was split between a quadrupole mass spectrometer and the electroantennographic detector in proportion of 1 to 1 . The interface was kept at a temperature of $230{ }^{\circ} \mathrm{C}$ and was equipped with an additional inlet for humidified synthetic air $\left(90 \%\right.$ R. H., $300 \mathrm{ml} \mathrm{min}^{-}$ 
${ }^{1}$ ) in order to modify effluent condition for compatibility with electroantennographic detection. Electrophysiological responses were measured by a high impedance amplifier. Details have been described in (Weißbecker et al., 2004). All measurements were made using at least five insect antennae.

\section{Solid-state sensor preparation and evaluation}

Tungsten oxide sensor films were prepared by applying a suspension of $\mathrm{WO}_{3}$ on an alumina substrate with interdigital platinum electrode. Prior to use the sensors were heated in a temperature-profile up to $450{ }^{\circ} \mathrm{C}$. We used special nanostructured $\mathrm{WO}_{3}$ powder as well as commercial $\mathrm{WO}_{3}$ powder (Sigma Aldrich). Nanostructured WO3 was synthesized by casting in nanoporous silica template as described by (Rossinyol et al., 2005). Silica templates of KIT-6 mesostructure were synthesized according to (Kleitz et al., 2003). Subsequently, 0.6g of KIT-6 was dissolved in ethanol $1.2 \mathrm{~g}$ phosphotungstic acid (PTA) was added as a $\mathrm{WO}_{3}$-precursor. Upon drying and calcination as described in (Rossinyol et al., 2005), which includes another 0.6g of PTA, the silica template was removed by etching with hydrofluoric acid (HF). Physisorption measurements revealed a specific surface area of $66 \mathrm{~m}^{2} / \mathrm{g}$. Scanning electron micrographs (SEM) of the sensor surface show nanostructured $\mathrm{WO}_{3}$ at different magnifications. The film consists of an irregular spread of nanostructured grains (left). The grains have a size of approximately $2-5 \mu \mathrm{m}$. The nanostructure is shown at the right of figure 1 .

A high resolution gas-chromatograph with parallel mass-spectrometric and solid state sensor detection (High resolution gas chromatography/selective odorant measurement by multisensor array, HRGC-SOMMSA) was used to evaluate responses of the sensors to volatile mixtures released by heated wood. The system is described in detail in (Kohl et al., 1998). We injected 0.5 microliter of the standard solution with a split ratio of $1: 4$ into a GC-MS system (Varian Saturn 2000) coupled to an adapted sensor chamber. The standard solution contains $1 \%$ of each marker volatile in ethanol. Helium was used as carrier gas at a flow rate of $5 \mathrm{~m} \mathrm{~min}^{-1}$. The effluent flow was split between an ion trap mass spectrometer and a sensor box in proportion of $1: 4$. The sensor chamber (inner volume 2 $\mathrm{ml}$ ) was at room temperature. Changes in sensor conductance were measured at a constant voltage of $0.5 \mathrm{~V}$. All measurements were made using at least two sensor elements of each type.
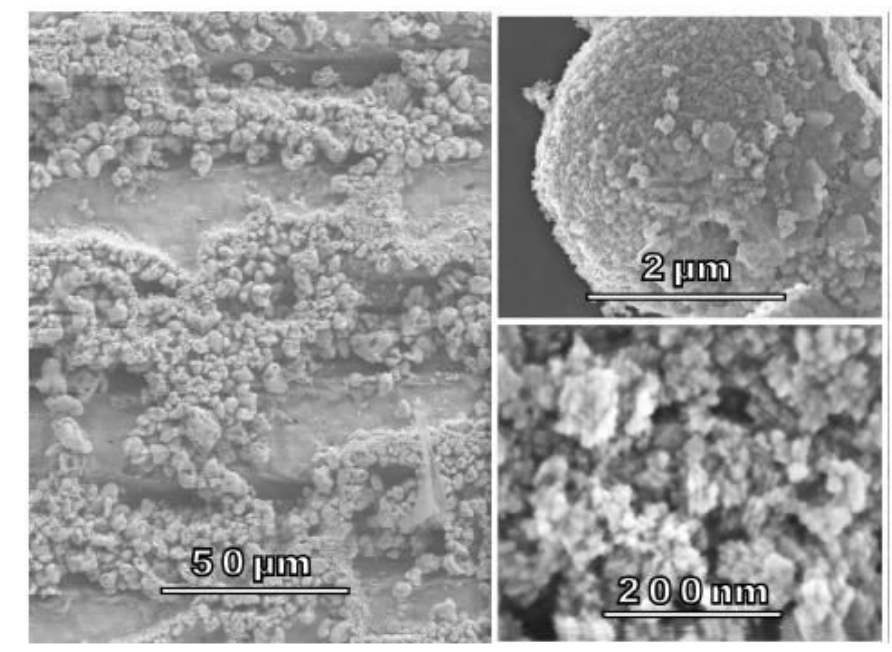

Figure 1: Nano-structured $\mathrm{WO}_{3}$ sensor films (SEM picture) 


\section{Results}

\section{Marker volatiles}

Despite separated development and different ecosystems, both beetles from Australia and from Europe show similar pattern of olfactory perception of forest fire volatiles. Both beetles show high electrophysiological responses to guaiacol and its derivatives, intermediate responses to syringol and its derivatives and least responses to coniferyl derivatives and benzenediols. Ortho- and parasubstituted methoxyphenoles show higher responses than meta- substituted methoxyphenoles. Host plant terpenoids are the second group of compounds detected with remarkable sensitivity. Whereas phenolic compounds are released at high temperatures due to the oxidative thermal degradation of lignin after ignition, terpenes are released at low to moderate temperature of the wood before ignition. Aldehydes from the thermal oxidation of fatty acids in the wood parenchyma are released as the first breakdown products of the wood composite structure. A fourth class of compounds released at increased heating temperatures but well before an ignition of a wood fire are decomposition products of celluloses and hemicelluloses, mainly comprising furane derivatives like furfural and furane breakdown products like hydroxyacetone. Using markers from these four groups allows a reliable assessment of the heating status of the trees and, therefore, can guide the beetles to an appropriately charred tree with enough heated wood left for bringing up their offspring. Electrophysiologcal and trace analytical data is presented in the manuscript "The olfaction of a fire beetle leads to new concepts for early fire warning systems", pp.103.

\section{Biomimetic solid-state sensor array}

We used nanostructured $\mathrm{WO}_{3}$ to achieve highly sensitive sensors (Figure 1). The comparison of nanostructured $\mathrm{WO}_{3}$ with bulk material reveals an increased sensitivity to the proposed marker substances. The sensitivity to hexanal, furfural and $\alpha$-pinene is shown in figure $2 . \mathrm{Bulk}^{\mathrm{W}} \mathrm{WO}_{3}$ sensor shows a response $(\Delta G / G-1)$ of 0.1 for furfural and $\alpha$-pinene respectively. The nanostructured sensor shows a response of 1.05 for furfural and 0.85 for $\alpha$-pinene. Figure 3 shows that sensitivity can be successfully increased by new nanostructuring methods. For the successful detection of smoldering wood fires especially for the detection of early stages a selective detection of several markers is required. This can be achieved with a sensor array in different ways. The most facile way is the usage of different semiconductor materials. A comparison of the sensitivity of the nanoporous $\mathrm{WO}_{3}$ sensors with the sensitivity reported by Eberheim (Eberheim 2003) for $\mathrm{SnO}_{2}$ sensors shows (Table 1) that a two sensor system should be feasible for the discrimination between early and late stages of wood fires. Previous research shows that selectivity can further be increased by various techniques, such as temperature (Eicker et al., 1981; Lalauze 1984; Gramm et al., 2002) or voltage (Sauerwald et al., 2007) cycled operation of the sensors. Preliminary results for nanostructured $\mathrm{WO}_{3}$ sensors show that a temperature between $350^{\circ} \mathrm{C}$ and $550^{\circ} \mathrm{C}$ is sufficient for the discrimination between furfural and $\alpha$ pinene (Figure 3). 


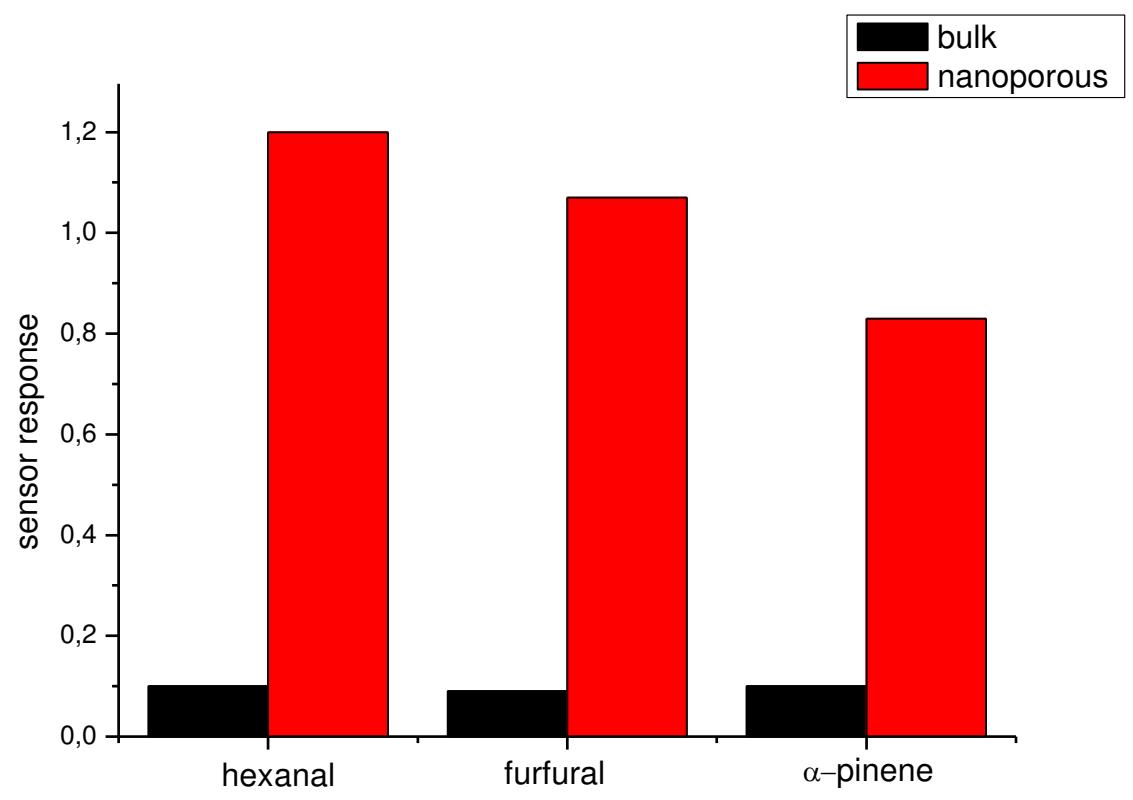

Figure 2: response of a nanostructured $\mathrm{WO}_{3}$ sensor and "bulk" $\mathrm{WO}_{3}$ sensor for three marker volatiles of heated wood. The marker volatiles are applied by GC-MS/SOMMSA method with $0.8 \mathrm{nl}$ of each volatile. Both sensors are heated to 500 ${ }^{\circ} \mathrm{C}$.

Table 1 Comparison of sensitivity of $\mathrm{WO}_{3}$ nanoporous sensors with the results for $\mathrm{SnO}_{2}$ sensors by Eberheim, 2003. Measurements were repeated twice (minimum). Signal intensity is classified as ++ strong, + medium, $\mathrm{X}$ weak or not significant. Signals of both sensor elements are reproducible in all measurements.

\begin{tabular}{|c|c|c|c|c|c|c|c|}
\hline Substance & 国/G & $\mathrm{SnO}_{2}$ & $\mathrm{WO}_{3}$ & Substance & 国/G & $\mathrm{SnO}_{2}$ & $\mathrm{WO}_{3}$ \\
\hline Guaiacol & & ++ & $x$ & Hexanal & & $-*)$ & ++ \\
\hline Furfural & & $x$ & ++ & $\alpha$-Pinene & & $x$ & ++ \\
\hline
\end{tabular}




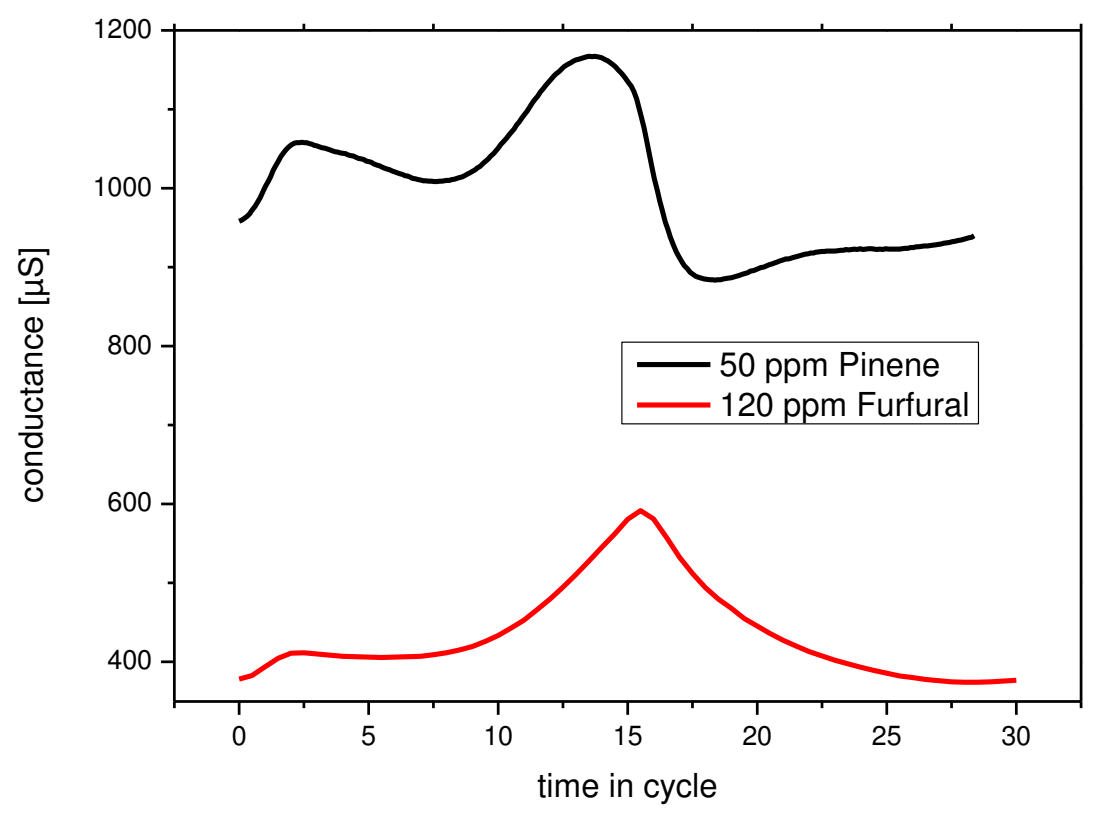

Figure 3: Conductance measurement of nanoporous $\mathrm{WO}_{3}$ sensor during a symmetric temperature cycle between $3500^{\circ} \mathrm{C}$ (at $\mathrm{t}=0$ and $\mathrm{t}=30$ resp.) and $550^{\circ} \mathrm{C}($ at $\mathrm{t}=15)$.

\section{Conclusions}

Phenolic compounds in wood smoke seem to play a predominant role in fire detection and discrimination of the examined pyrophilous beetles. These phenolic compounds are products of the oxidative thermal degradation of the biopolymer lignin; furans, aldehydes and ketones are degradation products of celluloses and fatty acids; and terpenoids are released by evaporation or degradation of secondary plant products like essential oils (Schütz et al., 1999c; Eberheim 2003). In different kinds of plants, lignin constitutes a bigger (trees) or smaller (grass, herbs) share of biomass and, moreover, differs in the structure of monomers contributing to the biopolymer. Whereas guaiacol is the major monomer contributing to lignin of coniferous wood, the wood of deciduous trees contains more syringol than guaiacol (Schütz et al., 1999a). Non-woody plants like grasses contain mainly lignin polymerized from coniferyl alcohols and benzenediols (Eberheim 2002). Upon thermal oxidation, lignin is broken down to fragments containing parts of these monomers. Thus, the constitution of biomass smoke reflects the constitution of the degrading lignin, enabling the pyrophilic insects to assess whether the fuel is a coniferous tree, a deciduous tree or only grassland. Differentiating guaiacol and syringol from para-substituted alkyl- or acyl derivatives enables the insect to assess the fire temperature, as with increasing temperature the breakdown products of lignin tend to become smaller. Detecting host plant terpenoids enables the insects to assess the suitability of the burning plant species as substrate for their offspring. Moreover, the ratio of phenolic compounds and terpenoids provides information if primarily wood or leaves of the respective plant species are burning, as burning fresh leaves or needles release higher quantities of terpenoids. Both, pyrophilic insects the European Melanophila acuminata and the Australian Merimna atrata, use a similar strategy for olfactory detection and classification of forest fires. Both of them detect guaiacol- derivatives and host plant terpenoids quite sensitively for long range orientation towards burning trees. Moreover, both of them do not only detect several guaiacol- and 
syringol-derivatives, but additional degradation products of cellulose and hemicelluloses like furane derivatives and hydroxyacetone which mark heated wood prior to ignition. Thus, a selection of a dozen of compounds from more than 200 detected volatile compounds in the forest fire samples is used by the beetles to extract vital information from highly diluted wood smoke.

These perception filters and evaluation algorithms can provide blue prints for biomimetic fire detection and fire early warning systems. Driven by these results, we focussed sensor development to the detection of specific groups of organic trace gases. Especially tin oxide and tungsten oxide sensor elements showed favourable characteristics. Their high sensitivity and selectivity to hydroxylated and phenolic compounds, and terpenes and furanes, respectively, in combination to low cross-reactivity with water and carbon monoxide mimics highly efficient biological fire detection systems. For sensor evaluation the HRGC/SOMMSA approach has proven to be very useful, as response time and sensitivity of the sensors are sufficient to use them as detectors for capillary gaschromatographic systems. A first practical application of these biomimetic sensors is already working as sensors for early warning systems in brown coal storage units (Eßinger et al., 2010) and is extended to wood chip storage units and wood chip driers of the wood processing industry.

\section{Acknowledgements}

Funding by the federal ministry of research and technology (BMBF) is gratefully acknowledged. The work of Sebastian Paczkowski is funded by the Cusanuswerk, Bischöfliche Studienstiftung, Germany.

\section{References}

Apel,K.-H. (1989) Zur Lebensweise des schwarzen Kiefernprachtkäfer Melanophila acuminata, Entomologische Nachrichten Berlin 33, 278-280.

Eberheim A. (2003) Qualifizierung von Halbleiter-Gassensoren für die Detektion spezifischer organischer Rauchgaskomponenten, Inauguraldissertation Inst. f. Angewandte Physik JLU Gießen.

Eicker,H., Kartenberg,H.J., Jacob,H. (1981) Untersuchung neuer Messverfahren mit Metalloxidhalbleitern zur Überwachung von Kohlenoxid-Konzentrationen, Technisches Messen 48, 421-430.

Eßinger,T.M., Becker,B., Sauerwald,T., Kohl,C.D., Paczkowski,S., Schütz,S. (2010) Volatile gas sensors for large-scale drying of wood. Proceedings Sensoren und Messsysteme 1, 780-783.

Gramm,A., Schütze,A., Rühl,T. (2002) Identification of organic solvents by a virtual multisensor system with hierarchical classification, Proceedings of the IEEE Sensors, 1, 382-387.

Kleitz,F., Choi,S.H., Ryoo,R. (2003) Cubic la3d large mesoporous silica: synthesis and replication to platinum nanowires, carbon nanorods and carbon nanotubes, Chemical Communications 17, 21362137.

Kohl,D., Schieberle,P. et al., (1998) High Resolution Gas Chromatography - Selective Odorant Measurement by Multi Sensor Array, Flavour Analysis, Chap. 29, American Chemical Society.

Kohl,D., Koczan,D. (1999) Application and perspective of biosensors based on insect olfaction, Proceedings Sensor 11, 105-110. 
Kuske,M. (2006) Detection of diverse mould species growing on building materials by gas sensor arrays and pattern recognition, Sensors \& Actuators B 119, 33-40

Lalauze,P. (1984) A new approach to selective detection of gas by a $\mathrm{SnO}_{2}$ solid-state sensor, Sensors and Actuators 5, $55-63$.

Presicce,D.S. (2006) Response evaluation of an E-nose towards contaminated wheat by Fusarium poae fungi, Sensors \& Actuators B 118, 433-438.

Rossinyol,E., Tian,B., et al., (2005) Nanostructured metal oxides synthesized by hard template method for gas sensing applications, Sensors and Actuators B 109, 57-63.

Sauerwald,T., Skiera,D., Kohl,C.D. (2007) Selectivity enhancement of gas sensors using nonequilibrium polarisation effects in metal oxide films, Applied Physics A 87, 3.

Schmitz,H., Schmitz,A. (2002) Australian fire beetles, Landscope 1, 36-41.

Schmitz,H., Mürtz,M., Bleckmann H. (2002) Infrared detection in a beetle Nature 386, 773-774.

Schütz,S., Weißbecker,B., Hummel,H.E., Apel,K.-H., Schmitz,H., Bleckmann,H. (1999a) Insect antennae as a smoke detector. Nature 398, 298-299.

Schütz S., Hummel H.E., Schöning M.J., Schroth P., Kordos P., Lüth H., Zimmermann S., Schwarz A. (1999b) Application and perspective of biosensors based on insect olfaction, Proceedings Sensor 11, 105-110.

Schütz,S., Weißbecker,B., Koch,U.T., Hummel,H.E., (1999c) Detection of volatiles released by diseased potato tubers using a biosensor on the basis of intact insect antennae, Biosensors \& Bioelectronics 14, 221-228.

Weißbecker,B., Holighaus,G., Schütz,S. (2004) Gas chromatography with mass spectrometric and electroantennographic detection:-analysis of wood odour by direct coupling of insect olfaction and mass spectrometry, Journal of Chromatography A 1056, 209-216.

Xavier,N. (2003) Odors in the food industry, Springer Verlag, Heidelberg, London \& New York, 61-122. 


\title{
Calliphora vicina (Rob.-Desv.) as an information filter for volatile-based meat spoilage detection
}

Authors names and affiliations

Corresponding author: Sebastian Paczkowski, Dept. of Forest Zoology and Forest Conservation, Büsgeninstitut, Georg-August-University, Büsgenweg 3, 37077 Göttingen, Germany.

Sara Nicke, Dept. of Forest Zoology and Forest Conservation, Büsgeninstitut, Georg-AugustUniversity, Büsgenweg 3, 37077 Göttingen, Germany. email: snicke@gwdg.de

Prof. Dr. Stefan Schütz, Dept. of Forest Zoology and Forest Conservation, Büsgeninstitut, GeorgAugust-University, Büsgenweg 3, 37077 Göttingen, Germany. email: stefan.schuetz@forst.unigoettingen.de

\begin{abstract}
The olfaction of the female blowfly was used to select volatiles that are reliably emitted by pork meat samples stored under warm/dry, warm/wet, cold/dry, and cold/wet conditions for meat quality assessment. The volatiles emitted by the samples were collected eight days with charcoal filters. 2,3Butanediol, hexanal, heptanal, dimethyl trisulfide, phenol, undecane, nonanal, and indole were identified and quantified by GC-MS. The volatiles were tested on the antenna of female $C$. vicina, which resulted in dimethyl trisulfide, nonanal, and indole as electrophysiological active marker compounds for meat spoilage. These compounds can be used for the identification of meat spoilage during meat maturation or as a sign for an interruption of the cooling chain.
\end{abstract}

Keywords: VOC, nonanal, pork meat spoilage, bacterial growth, blowfly, Electroantennography (EAG), gas sensor

\section{Introduction}

Meat quality is an important aspect in the supply chain from producer to customer. Although quality factors can be subjective, the microbial spoilage of meat is an objective health hazard. There are many different treatments that decrease bacterial growth on meat. Among them are packaging under anaerobic conditions, irradiation, washing, or treatment with inhibitors of bacterial growth (Reid et al., 1993; Cheng et al., 2011; Giri et al., 2011). The most wide spread method to prevent spoilage is cooling. Meat is usually stored at $5{ }^{\circ} \mathrm{C}$, as low temperature decreases enzymatic activity (Goll et al., 1983), which consequently suppresses bacterial metabolism. However, storage time is always limited and there are many studies concerning volatile based quality assessment of stored meat (Ercolini et al., 2009; Stutz et al., 1991; Mayr et al., 2003; Lindinger et al., 1998b; Lindinger et 
al., 1998a). Volatiles are good indicators for bacterial growth on meat, but the high variation of the volatile emissions impedes the transfer of the results from laboratory conditions to practical solutions (Winquist et al., 1993; Olafsdottir and Kristbergsson, 2006; Rajamaki et al., 2006; Paczkowski and Schütz, 2011). Different conditions might favor the growth of different bacterial communities, which emit different volatiles. Therefore, the identification of reliably emitted volatiles allowing an estimation of bacterial growth on meat will exceed the limits of conventional trace analytical studies. In this study, the biological optimization process of nature is used to identify volatiles that are reliably emitted during storage. The olfaction of the female blowfly Calliphora vicina, which is attracted by spoiled meat, was used to confirm the reliability of the detected volatile compounds. The evolution of the fly's olfaction led to an adaption to reliably emitted volatiles, as the female fly needs to find its nutrition and oviposition source by means of olfaction (Paczkowski and Schütz, 2011; Paczkowski et al., 2012). Four treatments were evaluated by this biological information filter. These treatments resembled the conditions of natural decomposition, meat maturation, and dry or wet storage in a refrigerator. The four different treatments allow a comparison of the investigated concept of spoilage detection between the control (natural decomposition) and the three artificial treatments (maturation and both cool storage conditions). This comparison assesses the feasibility of the fly's olfaction, which has been adapted to the natural conditions of vertebrate decay (Paczkowski and Schütz, 2011), for the artificial conditions during meat storage.

\section{Material and Methods}

\section{Collection of volatiles from decaying meat}

Four Longissimus dorsi of pork were purchased from a local butcher 2-4 hours after slaughtering. The pieces from the $11^{\text {th }}$ thoracic vertebra to the $2^{\text {nd }}$ lumbar vertebra were used for the experiments, because they show the lowest variation in the intramuscular fat content (Branscheid et al., 2007). Five $25 \mathrm{~g}$ samples of each $L$. dorsi were used for each treatment.

Two sets were stored at $25{ }^{\circ} \mathrm{C}$ and $20 \%$ rel.hum., one under dry conditions (warm/dry) and one set was sprinkled with $10 \mathrm{ml}$ water every day (warm/wet). Volatile samples were taken daily for eight days.

Two sets were stored at $5{ }^{\circ} \mathrm{C}$ and $35 \%$ rel.hum.. One set was kept under dry conditions (cold/dry) and the other set was sprinkled with $10 \mathrm{ml}$ water after each volatile sampling (cold/wet). From these two sets, volatile samples were taken nine times in one month.

For volatile sampling of one set, each of the five samples were put separately in a closed glass container with $0.5 \mathrm{~L}$ volume at $24 \pm 1{ }^{\circ} \mathrm{C}$. The containers had two openings, one entrance for filtered air and one exit for the air containing the volatiles from the sample. A membrane pump (Thomas Division, Sheboygan, USA) sucked $40 \mathrm{~L}$ in 30 min through the exit and through a charcoal filter tube (1.8 mg Charcoal, CLSA Filter, Daumazan sur Arize, France). 


\section{Trace analysis of the CLSA samples}

The trace analytical experiments were performed according to a method described in Paczkowski et al., 2012 (p.71).

\section{Quantification of volatiles from decaying meat}

The quantification was performed according to a method of Paczkowski et al., 2012 (p.71).

\section{Collection and rearing of flies}

In the summer of 2011, female C. vicina were caught from fresh ground meat (half pork, half beef) in the city of Göttingen. The species $C$. vicina was chosen as it is one of the first to arrive on a dead vertebrate, and is present during all stages of decay. This species is important for forensic entomology (Smith, 1986; Amendt et al., 2004) and is a model organism for research on the olfaction of blowflies (Kaib, 1974; Huotari and Mela, 1996; Stensmyr et al., 2002). After removing the antennae for electrophysiological experiments, the development of the ovaries was assessed (Stoffolano, 1974). This was used as an indicator of the predilection of the flies to oviposit and, therefore, exhibit high electrophysiological performance. Only flies with developed ovaries were selected for electrophysiological measurements.

\section{EAG with C. vicina}

Most compounds identified in the CLSA samples were tested in paraffin oil (Uvasol quality, Merck/VWR, Darmstadt, Germany) dilutions from $10^{-9} \mathrm{~g} / \mathrm{g}$ to $10^{-2} \mathrm{~g} / \mathrm{g}$. 2,3-butanediol and hexanal were tested in paraffin oil dilutions from $10^{-7}$ to $10^{-2}$. The EAG experiments were performed according to the method described in Paczkowski et al., 2012 (p.72).

\section{Results}

\section{Trace analysis and quantification of volatile samples from decaying meat}

The compounds emitted from the four different $L$. dorsi sets and detedcted in the trace analytical experiments are listed in Table 1 in the order of their retention index. Among the eight compounds identified and quantified there are one alkane (undecane), three aldehydes (hexanal, heptanal, nonanal), one alcohol (2,3-butanediol), one sulfur compound (dimethyl trisulfide), one homoaromatic compound (phenol), and one heteroaromatic compound (indole).

All of the four treatments showed a different odor profile (Table 2). The samples stored under warm conditions emitted more volatiles at higher concentrations than the samples stored under cold conditions, because both the vapor pressure of compounds and their biochemical formation processes depend on the temperature. The warm/dry treatment resulted in the maturation of the meat with the typical odor of dried ham (Toldra, 1998). These samples emitted nonanal at relatively high rates and hexanal, heptanal, and undecane at low rates. The warm/wet samples developed a pungent smell, showed a greenish surface and deliquesced during the nine days of sampling. They exclusively emitted phenol and indole at high concentration and dimethyl trisulfide at low concentration and hexanal, nonanal, undecane, and 2,3-butanediol. The cold/dry samples dried out 
Table 1: Identified volatiles. The table shows the compounds of the $L$. dorsi samples detected in the trace analytical studies in the order of their retention index and assigned to their chemical classes. Information on the CAS number, the company from which the compound was purchased and its purity, the vapor pressure in ppm and the ion that was used for quantification of the compound is listed.

\begin{tabular}{|c|l|c|l|c|c|c|c|c|}
\hline No. & Compound & $\begin{array}{c}\text { HP- } \\
\text { 5MS } \\
\text { (LRI) }\end{array}$ & \multicolumn{1}{|c|}{$\begin{array}{c}\text { Chemical } \\
\text { class }\end{array}$} & CAS No. & Company & $\begin{array}{c}\text { Purity } \\
{[\%]}\end{array}$ & $\begin{array}{c}\text { Vapor } \\
\text { pressure } \\
\text { [hPa]* }\end{array}$ & $\begin{array}{c}\text { Ion for } \\
\text { quanti- } \\
\text { fication } \\
\text { [m/z] }\end{array}$ \\
\hline 1 & 2,3 -Butanediol & $<800$ & Alcohol & $19132-06-0$ & Aldrich & 98 & 0.22 & 45 \\
\hline 2 & Hexanal & 826 & Aldehyde & $66-25-1$ & Aldrich & 98 & 12 & 44 \\
\hline 3 & Heptanal & 910 & Aldehyde & $111-71-7$ & Arcos & 95 & 3 & 44 \\
\hline 4 & Dimethyl trisulfide & 979 & Sulfur compound & $3658-80-8$ & SAFC & 98 & 0.35 & 126 \\
\hline 5 & Phenol & 995 & $\begin{array}{l}\text { Homoaromatic } \\
\text { compound }\end{array}$ & $108-95-2$ & Aldrich & 99 & 0.2 & 94 \\
\hline 6 & Undecane & 1101 & Alkane & $1120-21-4$ & Arcos & 99 & 0.6 & 57 \\
\hline 7 & Nonanal & 1118 & Aldehyde & $124-19-6$ & Merck & 98 & 0.5 & 57 \\
\hline 8 & Indole & 1211 & $\begin{array}{l}\text { Heteroaromatic } \\
\text { compound }\end{array}$ & $119-36-8$ & Fluka & 99 & 0.001 & 120 \\
\hline
\end{tabular}

* measured at $20^{\circ} \mathrm{C}$ and $1013 \mathrm{hPa}$

slowly and were completely hardened at the end of the sampling period. The samples emitted only 2,3-butanediol at high rates and nonanal at low rates. The cold/wet samples emitted 2,3-butanediol and nonanal at rates comparable to the cold/dry samples. Although the samples were very moist they did not deliquesced during the sampling period.

The consistency of the volatile profiles between the samples is restricted to nonanal and 2,3 butanediol, which are present in all meat samples. This shows the strong influence of the treatments on the identified volatile profile and indicates the restriction of the transfer from warm conditions to which C. vicina is adapted, to the cooler conditions during meat storage. 
Table 2: Quantification. The table shows the emission rates ( $\mathrm{ng} / \mathrm{L}$ ) of the compounds identified and quantified in the different treatments of the meat samples ( $\mathrm{N}=5$ for each treatment). They

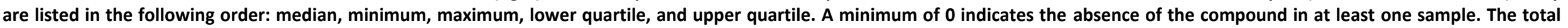
emission is the sum of the medians.

\begin{tabular}{|c|c|c|c|c|c|c|c|c|}
\hline Day & 1 & 2 & 3 & 4 & 5 & 6 & 7 & 8 \\
\hline \multicolumn{9}{|l|}{ L. dorsi (warm/dry) } \\
\hline 2,3-Butanediol & - & - & - & - & - & $0,0,1,0,0$ & $0,0,1,0,0$ & $0,0,3,0,0$ \\
\hline Hexanal & $0,0,1,0,0$ & - & $0,0,1,0,1$ & $1,0,1,1,1$ & $1,0,1,0,1$ & $1,1,2,1,2$ & $1,1,1,1,1$ & $2,1,2,1,2$ \\
\hline Heptanal & - & $0,0,1,0,0$ & - & $0,0,1,0,1$ & - & $0,0,1,0,0$ & $0,0,1,0,0$ & $1,1,2,1,1$ \\
\hline Dimethyl trisulfide & $1,0,1,1,1$ & - & - & - & - & - & $0,0,1,0,0$ & - \\
\hline Undecane & $1,0,1,0,1$ & $1,0,3,1,1$ & - & - & $0,0,1,0,1$ & $1,0,1,0,1$ & $1,0,1,0,1$ & $1,0,1,1,1$ \\
\hline Nonanal & $47,26,71,43,55$ & $32,11,58,31,36$ & $52,35,56,45,56$ & $55,26,61,35,59$ & $26,16,26,25,26$ & $26,23,31,25,29$ & $17,16,24,17,21$ & $41,33,50,36,42$ \\
\hline Total emission & 49 & 33 & 52 & 56 & 27 & 28 & 19 & 45 \\
\hline \multicolumn{9}{|l|}{ L. dorsi (warm/wet) } \\
\hline 2,3-Butanediol & - & - & $1,0,4,0,2$ & $104,2,304,2,255$ & $158,15,216,27,181$ & $191,32,289,54,243$ & $261,40,735,69,305$ & $95,26,531,40,531$ \\
\hline Hexanal & $1,1,2,1,2$ & $1,0,1,0,1$ & $0,0,1,0,1$ & - & - & - & - & - \\
\hline Dimethyl trisulfide & - & - & - & - & $1,1,5,1,2$ & $1,0,1,1,1$ & $0,0,1,0,1$ & $1,1,1,1,1$ \\
\hline Phenol & - & - & - & $3,2,257,2,130$ & $146,82,292,85,172$ & $296,254,437,274,302$ & $287,183,298,231,290$ & $190,132,333,187,198$ \\
\hline Undecane & $1,1,2,1,2$ & - & - & - & $1,0,1,1,1$ & $0,0,1,0,1$ & $0,0,1,0,0$ & $0,0,1,0,0$ \\
\hline Nonanal & $3,2,3,2,3$ & $2,1,3,2,2$ & $2,1,4,1,3$ & $1,0,1,0,1$ & $1,0,1,0,1$ & - & - & - \\
\hline Indole & - & - & - & $4,0,45,0,27$ & $9,0,34,3,17$ & $48,11,76,48,67$ & $41,7,66,28,46$ & $38,6,72,31,47$ \\
\hline Total emission & 5 & 3 & 3 & 112 & 306 & 536 & 589 & 324 \\
\hline Day & 1 & 4 & 9 & 11 & 16 & 18 & 23 & 25 \\
\hline \multicolumn{9}{|l|}{ L. dorsi (cold/dry) } \\
\hline 2,3-Butanediol & - & - & $4,0,12,0,5$ & $28,0,57,0,44$ & $28,20,85,21,50$ & $34,23,83,26,76$ & $34,26,61,32,44$ & $34,20,54,25,40$ \\
\hline Nonanal & $1,0,2,0,1$ & $3,0,6,2,3$ & $2,1,2,1,2$ & $3,2,4,2,3$ & $0,0,1,0,0$ & $0,0,1,0,1$ & $1,0,2,1,1$ & $1,1,1,1,1$ \\
\hline Total emission & 1 & 3 & 6 & 31 & 28 & 34 & 35 & 35 \\
\hline Day & 1 & 3 & 8 & 10 & 15 & 17 & 22 & 24 \\
\hline \multicolumn{9}{|l|}{ L. dorsi (cold/ wet) } \\
\hline 2,3-Butanediol & - & - & $0,0,1,0,1$ & $4,0,58,1,58$ & $23,8,131,23,125$ & $55,24,139,28,99$ & $21,9,65,16,40$ & $25,16,73,17,31$ \\
\hline Nonanal & $5,3,7,5,5$ & $5,3,6,5,5$ & $10,7,14,7,12$ & $5,1,16,3,8$ & $6,1,12,6,8$ & $1,1,2,1,1$ & $3,2,7,2,6$ & $2,1,3,1,3$ \\
\hline Total emission & 5 & 5 & 10 & 9 & 29 & 56 & 24 & 27 \\
\hline
\end{tabular}




\section{EAG with C. vicina}

The results of the EAG experiment have been published in Paczkowski et al., 2012 (p.71). Additional data is presented in Table 3.

Table 3: EAG. The table shows the electrophysiological response of $C$. vicina females towards hexanal and 2,3-butanediol. The vapor pressure of the first significant dilution is calculated in order to compare both compounds. A low vapor pressure indicates a strong response of the insect. Further electrophysiological data has been published in Paczkowski $e t$ al., 2012.

$\begin{array}{lccccccc} & \begin{array}{c}\text { Paraffin } \\ \text { oil }\end{array} & 10^{-6} & 10^{-5} & 10^{-4} & 10^{-3} & 10^{-2} & \begin{array}{c}\text { Vapor pressure of } \\ 1^{\text {st }} \text { significant } \\ \text { dilution [ppm] }\end{array} \\ \text { Hexanal } & & & & & & & \\ & 11(9,13) & 12(11,14) & 13(13,18) & 20(19,28) & 33(31,57) & 78(73,124) & 14 \\ \text { 2,3-Butanediol } & 10(9,12) & 11(9,14) & 10(9,13) & 10(9,11) & 11(10,12) & 18(13,18) & 90\end{array}$

\section{Discussion}

The samples stored under warm conditions did emit more volatiles than the samples stored under cold conditions, because low temperatures inhibited the bacterial growth (Goll et al., 1983) and decreased the volatility of the compounds (Thomson, 1946). The differences in the volatile emission pattern between the wet and the dry treatments can be explained by the moisture that increases bacterial growth (Gottschalk, 1986) and thereby increases the volatile formation.

Nonanal is the only compound that is reliably present in all four treatments and it is perceived by the fly at a low vapor pressure of the first significant concentration (Paczkowski et al., 2012). Nonanal is emitted at high rates during the warm/dry treatment (Table 2), which resembled meat maturation by autolysis (Toldra, 1998). This shows that an exclusive emission of nonanal and other aldehydes are a reliable sign for the correct maturation of meat by autolytic processes. As the emission of 2,3butanediol is linked to the growth of Pseudomonas (Mayr et al., 2003, Ercolini et al., 2009), the compound could, together with nonanal, be a reliable marker for an inappropriate maturation of the meat or of spoilage under cool conditions (Table 2). The emission of nonanal decreases under the increase of 2,3-butanediol emission, which indicates an inhibition of autolytic processes by bacterial growth. Microorganisms will most probably utilize carbohydrates as the first and most feasible source of energy, which yields 2,3-butanediol or other products of carbohydrate fermentation (Paczkowski and Schütz, 2011). However, C. vicina does not perceive 2,3 butanediol, as the fly is optimized for the detection of cadavers in the vegetation period and does not perceive volatiles emitted under cold conditions as they are in hibernation at these temperatures (Matuszewski et al., 2013).

Several compounds are uniquely detected for meat stored under warm and wet conditions. Dimethyl trisulfide forms from methanethiol (Frederick et al., 1957), which is a product of cysteine and methionine (Paczkowski and Schütz, 2011), and is a sign for advanced deterioration of meat (Table 2). The formation of sulfur compounds can be linked to anaerobic and aerobic bacterial species 
(Dainty et al., 1989; Ercolini et al., 2009). The antiseptic compound phenol is a compound associated with advanced deterioration, as well. It could be emitted by phenol-resistant bacteria who metabolize tyrosine (Paczkowski and Schütz, 2011) in order to compete against non-resistant species (Gu et al., 2007). The meat pieces were small and a fast growth of different bacterial species would lead to bacterial competition. Indole forms from tryptophan and is a reliable marker for bacterial growth (Smith and Macfarlane, 1996). Despite its low vapor pressure (Table 1) it is detected at high rates. As dimethyl trisulfide and indole are perceived by female $C$. vicina at low vapor pressure (Paczkowski et al., 2012), both compounds are regarded as marker compounds for meat spoilage under natural conditions. Therefore, the additional emission of dimethyl trisulfide and indole together with 2,3-butanediol and nonanal can be a sign for a discontinuous cold chain that led to increasing growth of mesophilic bacteria. Metal oxide gas sensors will have to be tuned to the selective detection of these four volatiles. Temperature cycles (Gramm and Schütze, 2003; Paczkowski et al., 2011; Semancik et al., 2001), nanostructured thin layer sensors (Kakati et al., 2009) or $\mu$ GCs (Kim et al., 2012) can improve the selectivity and sensitivity of these gas sensors, however, it has to be taken in account that the olfaction of the fly even exceeds the detection treshhold of a GCMS system. Although the sensitivity of gas sensors are in the same order of magnitude as GC-MS systems, their selectivity is much lower. This results in a challenging task to adapt gas sensors to compounds perceived by $C$. vicina.

\section{Conclusion}

The volatile emission patterns analyzed in this study are correlated to the treatment of the meat samples. Nonanal is detected by C. vicina and can be used together with dimethyl trisulfide, indole and 2,3 butanediol for a reliable differentiation between maturation and deterioration of meat. As $C$. vicina is adapted to perceive a dead vertebrate during the warmer growth season, the olfaction of the fly is restricted to the detection of spoilage under warm conditions. However, the emission of volatiles detected by the fly can indicate a disruption of the cooling chain, which can be of importance for the meat trading sector. This study further indicates that 2,3-butanediol is a good candidate for spoilage detection under cold conditions.

\section{Acknowledgements}

The work of Sebastian Paczkowski was kindly supported by the Cusanuswerk, Bischöfliche Studienstiftung. The work of Sara Nicke was kindly supported by the BMBF, Germany 


\section{References}

Amendt,J., Krettek,R. and Zehner,R. (2004) Forensic entomology. Naturwissenschaften, 91, 51-65.

Branscheid,W., Honikel,K.O., v.Lengerken,G., and Troeger,K. (2007) Qualität von Fleisch- und Fleischwaren. Deutscher Fachverlag GmbH, Frankfurt am Main, Germany.

Castello,G., Moretti,P. and Vezzani,S. (2009) Retention models for programmed gas chromatography. Journal of Chromatography A, 1216, 1607-1623.

Cheng,A.W., Wan,F.C., Xu,T.C., Du,F.L., Wang,W.L. and Zhu,Q.J. (2011) Effect of irradiation and storage time on lipid oxidation of chilled pork. Radiation Physics and Chemistry, 80:475-480.

Dainty,R.H., Edwards,R.A., Hibbard,C.M. and Marnewick,J.J. (1989) Volatile compounds associated with microbial-growth on normal and high $\mathrm{pH}$ beef stored at chill temperatures. Journal of Applied Bacteriology, 66, 281-289.

Ercolini,D., Russo,F., Nasi,A., Ferranti,P. and Villani,F. (2009) Mesophilic and psychrotrophic bacteria from meat and their spoilage potential in vitro and in beef. Applied and Environmental Microbiology, 75, 1990-2001.

Frederick,L.R., Starkey,R.L. and Segal,W. (1957) Decomposability of some organic sulfur compounds in soil. Proceedings of the Soil Science Society of America, 21, 287-292.

Giri,A., Osako,K., Okamoto,A., Okazaki,E. and Ohshima,T. (2011) Effect of meat washing on the development of impact odorants in fish miso prepared from spotted mackerel. Journal of the Science of Food and Agriculture, 91:850-859.

Goll,D.E., Otsuka,Y., Nagainis,P.A., Shannon,J.D., Sathe,S.K. and Muguruma,M. (1983) Role of muscle proteinases in maintenance of muscle integrity and mass. Journal of Food Biochemistry, 7, 137-177.

Gottschalk,G. (1986) Bacterial metabolism. Springer Verlag, New York, USA.

Gramm,A. and Schütze,A. (2003) High performance solvent vapor identification with a two sensor array using temperature cycling and pattern classification. Sensors and Actuators B-Chemical, 95, 5865.

Gu,Y.Q., Mo,M.H., Zhou,J.P., Zou,C.S. and Zhang,K.Q. (2007) Evaluation and identification of potential organic nematicidal volatiles from soil bacteria. Soil Biology \& Biochemistry, 39, 2567-2575.

Huotari,M. and Mela,M. (1996) Blowfly olfactory biosensor's sensitivity and specificity. Sensors and Actuators B-Chemical, 34, 240-244.

Kaib,M. (1974) Receptors for meat-odor and flower-odor on antenna of blowfly Calliphora-vicina. Journal of Comparative Physiology, 95, 105-121. 
Kakati,N., Jee,S.H., Kim,S.H., Lee,H.K., Yoon,Y.S. (2009) Sensitive enhancement of ZnO nanorod gas sensors with surface modification by an InSb thin film. Japaneese Journal of Applied Physics. 48, 105002.

Kim,S.K., Chang,H.W., Zellers,E.T. (2011) Microfabricated gas chromatograph for the selective determination of trichloroethylene vapor at sub-parts-per-billion concentrations in complex mixtures. Analytical Chemistry, 83:7198-7206.

Lindinger,W., Hansel,A. and Jordan,A. (1998a) On-line monitoring of volatile organic compounds at pptv levels by means of proton-transfer-reaction mass spectrometry (PTR-MS) - Medical applications, food control and environmental research. International Journal of Mass Spectrometry, 173, 191-241.

Lindinger,W., Hansel,A. and Jordan,A. (1998b) Proton-transfer-reaction mass spectrometry PTR-MS: on-line monitoring of volatile organic compounds at pptv levels. Chemical Society Reviews, 27, 347354.

Matuszewski,S., Szafalowicz,M., Grzywacz,A. (2013) Temperature-dependent appearance of forensically useful flies on carcasses. International Journal of Legal Medicine, DOI 10.1007/s00414013-0921-9.

Mayr,D., Margesin,R., Klingsbichel,E., Hartungen,E., Jenewein,D., Schinner,F. and Märk,T.D. (2003) Rapid detection of meat spoilage by measuring volatile organic compounds by using proton transfer reaction mass spectrometry. Applied and Environmental Microbiology, 69, 4697-4705.

Olafsdottir,G. and Kristbergsson,K. (2006) Electronic-nose technology, Application for quality evaluation in the fish industry. In Nicolay,X. (ed.), Odors in the food industry. Springer, pp. 57-74.

Paczkowski,S. and Schütz,S. (2011) Post-mortem volatiles of vertebrate tissue. Applied Microbiology and Biotechnology 91, 917-935.

Paczkowski,S., Sauerwald,T., Weiß.A., Bauer,M., Kohl,C.-D., Schütz,S. (2011) Biomimetic gas sensors for large-scale drying of wood particles. Martin-Palma, R. J. and Lakhtakia, A. 7975, 797505-7975058. Conference Proceedings SPIE: Bioinspiration, Biomimetics, and Bioreplication, San Diego, USA.

Paczkowski, S., Paczkowska, M., Maibaum, F., and Schütz, S. (2012) Decaying mouse volatiles perceived by Calliphora vicina Rob.-Desv. Journal of Forensic Sciences. 57, 1497-1506.

Rajamaki,T., Alakomi,H.L., Ritvanen,T., Skytta,E.S., Smolander,M. and Ahvenainen,R. (2006) Application of an electronic nose for quality assessment of modified atmosphere packaged poultry meat. Food Control, 17, 5-13.

Reid,D.H., Young,O.A. and Braggins,T.J. (1993) The effects of antioxidative treatments on mutton flavor odor intensity and species flavor differentiation. Meat Science, 35:171-182.

Schütz,S., Weissbecker,B., Koch,U.T. and Hummel,H.E. (1999) Detection of volatiles released by diseased potato tubers using a biosensor on the basis of intact insect antennae. Biosensors and Bioelectronics, 14, 221-228. 
Semancik,S., Cavicchi,R.E., Wheeler,M.C., Tiffany,J.E., Poirier,G.E., Walton,R.M., Suehle,J.S., Panchapakesan,B. and DeVoe,D.L. (2001) Microhotplate platforms for chemical sensor research. Sensors and Actuators B Chemical, 77, 579-591.

Smith,K.G.V. (1986) A manual of forensic entomology. Department of Entomology, British Museum (Natural History), London, United Kingdom.

Smith,E.A., Macfarlane,G.T. (1996) Enumeration of human colonic bacteria producing phenolic and indolic compounds: effects of $\mathrm{pH}$ carbohydrate availability and retention time on dissimilatory aromatic amino acid metabolism. Journal of Applied Bacteriology, 81, 288-302.

Stensmyr,M.C., Urru,I., Collu,I., Celander,M., Hansson,B.S. and Angioy,A.M. (2002) Rotting smell of dead-horse arum florets - These blooms chemically fool flies into pollinating them. Nature, 420, 625626.

Stoffolano,J.G. (1974) Influence of diapause and diet on development of gonads and accessory reproductive glands of black blowfly, Phormia-regina (Meigen). Canadian Journal of Zoology-Revue Canadienne de Zoologie, 52, 981-991.

Stutz,H.K., Silverman,G.J., Angelini,P. and Levin,R.E. (1991) Bacteria and volatile compounds associated with ground-beef spoilage. Journal of Food Science, 56, 1147-1153.

Toldra,F. (1998) Proteolysis and lipolysis in flavour development of dry-cured meat products. Meat Science, 49, S101-S110.

Thomson,G. (1946) The antoine equation for vapor-pressure data. Chemical Reviews, 38, 1-39.

Weissbecker,B., Holighaus,G. and Schütz,S. (2004) Gas chromatography with mass spectrometric and electroantennographic detection: analysis of wood odorants by direct coupling of insect olfaction and mass spectrometry. Journal of Chromatography A, 1056, 209-216.

Winquist,F., Hornsten,E.G., Sundgren,H. and Lundstrom,I. (1993) Performance of an electronic nose for quality estimation of ground meat. Measuring Science and Technology. 4, 1493-1500. 


\section{General Discussion}

This discussion is devoted to the general discussion of the methods used for this thesis and to the evaluation of the hypothesis, which was that insect olfaction can be used as an information filter for analytical applications. In detail the three hypothesesis, which should be verified in this thesis, were:

- Feasibility of the olfaction of Calliphora vicina for post mortem interval (PMI) estimation

- Feasibility of the olfaction of different fire beetles for early fire warning

- Feasibility of $C$. vicina for meat spoilage detection

Considering both aspects (method and hypothesis) is the basis of scientific work. Any method has its limitations, which defines the informative value of the results and this has to be considered when interpreting the results. This can be of high importance for the evaluation of a hypothesis. It is possible that experiments yield repeatable results, but these results cannot be related to natural conditions, because the method used does not resemble these natural conditions.

For instance, sampling volatiles at a constant temperature in a laboratory experiment enables a rather precise determination of the emission rate, but this rate is of little use for an estimation of the emission rate under natural conditions (DeGreef and Furton, 2011). Sampling the same volatiles under natural temperature variation restricts the exact prediction of emission rates (Statheropoulos et al., 2005; Paczkowski et al., 2012a), because the rate of volatile formation and its emission depend on the temperature in a non linear way (Goll et al., 1983; Giancoli, 2010). The combination of lab results and field studies can improve the limitations of both approaches. In general, it is necessary to design more than one experiment in order to verify scientific information by getting different "points of view" on this information (personal communication, Prof. Dr. Angeli, 2008). When all points of view (= all experiments) lead to the same result, this result can be regarded as confirmed. Chemoecology offers three points of view. The first is the trace analytical analysis. The second step is the identification of electrophysiological active compounds and the third step is the behavioral study in the lab or in the field (Schütz, 2009).

\section{Discussion of the Methods}

Table 1 shows the projects of this thesis and the methodical steps applied for the projects. Only those experiments are listed in the table, which were considered for publication. Unsuccessful experiments with methodological problems or unexplanatory results were excluded.

None of the chemoecological projects in this thesis comprise all methodological steps. In general, one has to conclude that all these missing methods should be performed in order to verify the project's hypothesis. 
Table 1 shows the experimental steps successfully performed for the chemoecological projects of this thesis. Gas sensor experiments, which were performed by colleagues at the Institute of Applied Physics in Gießen are not listed.

\begin{tabular}{|l|c|c|c|c|}
\hline Project & Trace analysis & Electrophysiology & Behavioral study & Field experiment \\
\hline C. vicina - meat & $\mathrm{X}$ & $\mathrm{X}$ & & \\
\hline C. vicina - cadaver & $\mathrm{X}$ & $\mathrm{X}$ & $\mathrm{X}$ & \\
\hline M. cuspidata - fire & $\mathrm{X}$ & $\mathrm{X}$ & $\mathrm{X}$ & \\
\hline M. atrata - fire & $\mathrm{X}$ & & $\mathrm{X}$ & $\mathrm{X}$ \\
\hline A. nigricans - fire & & & & \\
\hline
\end{tabular}

However, it is not always possible to acquire all of the data necessary for a complete chemoecological study. Behavioral studies with $C$. vicina led to no satisfactory results, because no wind tunnel was available (Aak et al., 2010) and the experiments were performed in a custom build Y-olfactometer where the flies had to walk and could not fly. Time consuming field tests on Melanophila cuspidata could be a topic for another doctoral thesis. Comprehensive behavioral studies with Merimna atrata could not be performed because of the lack of time and resources in Australia and the weakness of the beetles after the transport to Germany. The field tests with $M$. atrata showed only a slight tendency of a higher attractivity of odor baited traps in comparison to the paraffin control. Trace analysis for Acanthocnemus nigricans was not performed, as the results from both the trace analysis for $M$. cuspidata and $M$. atrata already suggested key volatiles emitted by smoldering logs, which were successfully tested in behavioral studies on $A$. nigricans. Comprehensive electrophysiological experiments with $A$. nigricans failed because of low antennal responses with low reproducibility in the EAG. Although there are good reasons for not having performed all of the chemoecological steps, this causes restrictions for the interpretation of the data. This is considered in the discussions of the manuscripts written for this doctoral thesis.

\section{Trace analysis}

\section{Compound identification}

The identity of a volatile compound was confirmed by two GC-MS methods in this doctoral thesis. The first method is the confirmation by the retention time in a GC-MS analysis and the second is the comparison of the mass spectra with a database (Hübschmann, 2001). In order to apply the first method, a compound is purchased and analyzed with the same GC-MS parameters as the sample. For the second method, databases can be purchased (Watson et al., 2004). If the retention time of this standard compound is the same as the retention time in the sample, the vapor pressure is the same for both compounds. However, it is still possible that there exist two or more compounds with the same vapor pressure or that the combination of vapor pressure and column properties result in the same retention time for two compounds. For instance, on a polar column a compound with a low vapor pressure and low polarity might reach the detector at the same time as a compound with higher vapor pressure and higher polarity. This leads to a mixed mass spectrum, which disables precise identification by MS. In such cases, a variation of the analytical parameters, e.g. the choice of column or the temperature program, can improve compound separation (McNair and Miller, 1997). Modern approaches already combine methods during one analytic procedure, like combined columns (GCXGC, two dimensional gas chromatography) (Kalinova et al., 2009) or combined quadrupole mass spectrometer (MS/MS, tandem mass spectrometry) (Müller et al., 2002) or by combining both (GCXGC-MS/MS) (Poliak et al., 2008) to enhance the analytical performance. TOF 
(time of flight) mass spectrometers (Kim and Kim, 2012) or triple quadrupole mass spectrometer (Yoon et al., 2012) are among the latest inventions for increased precision.

Another important point to consider when interpreting a GC-MS chromatogram is the artifact formation. In this context the term "artifact" is used for any compounds or ions that form during the whole analysis from sampling (Adams et al., 2012; Lestremau et al., 2004) to the detector plate of the MS (Jones and Chalmers, 2000; Andersen et al., 2012). Of course, most artifact formations do not influence the chromatogram to a high extent, but they have to be considered in order to assess the limits of the method. The first source of artifacts is the volatile sampling on an adsorbent. All kinds of chemical groups can occur as volatiles and it is probable that these groups react with each other on the adsorbent, which leads to the formation of different chemical compounds (Adams et al., 2012). A long interval between sampling and injection of the sample contributes to this kind of artifact formation. Especially the sunlight with the resulting raise of the temperature and UV induced radical formation during direct exposition causes chemical reactions on the adsorbents. The usage of passive volatile traps, which are deposited for a long period in the field, can lead to such an artifact formation (Dekeirsschieter et al., 2009). Furthermore, the adsorbents itself can degrade and cause artifact formation (Clausen and Wolkoff, 1997).

The second source of fragments is the analysis by GC-MS. During this process the volatiles in the sample are exhibited to highly artificial conditions, including high temperature, high concentrated solvents, reaction inducing surfaces, or ionization. For instance, it has been observed that furfural solved in ethanol turns to a different compound, possibly a ketone, with a higher retention time. The quantitative relation of both compounds change during storage (Personal communication, Dr. Gottschald, 2009). Furthermore, liquid injection or thermo desorption are usually performed at high temperatures (see Introduction, Identification of VOCs, Injector). These temperatures favor the reaction between compounds in the sample (Atkins and Beran, 2006), which has been shown by a study on artifact formation during SPME-GC-MS analysis with relatively unstable sulfur compounds. The study demonstrates that the sulfur compounds sampled on SPME and desorbed in a high temperature injection port change their structure (Lestremau et al., 2004). Own studies on dimethyl trisulfide showed three peaks for this compound in one chromatogram at time intervals of 0.5 and 2 minutes (Paczkowski, 2008). This result, which is a contradiction to the principle of gaschromatography, gave rise to the interpretation that a formation of dimethyl trisulfide from other sulfur containing compounds took place. As all compounds are located at the beginning of the column prior to separation, the rising temperature can induce chemical reactions on unstable compounds, like methanethiol, which would react to more stable compounds, like methylated sulfides. It has already been shown, that sulfides are more stable than other sulfur compounds (Lestremau et al., 2004; Paczkowski and Schütz, 2011) and it is most probable that the second and the third peak of dimethyl trisulfide are products of a chemical reaction induced by the temperature program of the GC oven during analysis (Paczkowski, 2008). Even octaatomic sulfur, which is the most stable compound among the sulfur compounds, was detected. These results support the hypotheses that the column in the GC oven can be regarded as a small reaction chamber. Another source of artifacts is the ionization of the compounds in the mass spectrometer. lonized fragments can form different structures by reacting with each other. If two compounds are overlapping in a chromatogram, this effect will influence the mixed spectrum and might disable an interpretation of the two mass-spectra (Maire and Lange, 2010). 


\section{Compound quantification}

After the identification of a volatile compound it can be quantified. Quantification can be performed in different grades of exactness. The lowest grade of exactness is reached by using the quantity of the standard compound as a single reference. For exacter quantification, the standard compound can be diluted at different concentrations in order to calculate a calibration curve based on the different peak areas (Steffen and Pawliszyn, 1996). It has to be considered that quantification should be compound specific. Quantifying the MS peak areas of two different compounds by using the same standard compound will distort the results, because the fragmentation is compound specific. Even in the single ion mode every compound has to be quantified with its own standard compound. The FID offers the possibility to use one standard compound peak area for the quantification of all carbon compounds. However, it has to be considered that the same concentration of two compounds with different mol weight can result in different peak areas. Empirically determined response factors can be used to compare the peak areas of different compounds with each other (Tong and Karasek, 1984).

Despite the discussed limitations of the analytical devices, it should always be considered that the quality of quantification highly depends on the bias of the sampling method and the sample preparation. While analytical devices and correct data processing allow a quite exact determination of a compound's quantity in a sample, the sampling method can show a low quantitative reproducibility (Paczkowski and Schütz, 2011). The reliability of the sampling method can be increased by a repetitive sampling, the use of an internal standard, and the calibration of the adsorbent (Tholl et al., 2006). High repetition and internal standard can only increase the exactness of the quantitative analysis, but the calibration of the adsorbents enables the calculation of emission rates. An adsorbent tube has to be exposed to a headspace that contains a standard compound, which emits from a prepared solution. The adsorbed amount depends on the emission rate of the compound and of the adsorbents properties of the tube. This amount can be compared with a detected amount in a sample and can be used for the estimation of the emission rate. Another possibility to calculate the emission rate is the injection of a defined amount of a standard compound into the adsorbents tube. Airflow transports the compound through the tube and part of it is adsorbed, while the other part leaves the tube. The resulting peak area takes in account the loss of molecules which had not been adsorbed in the tube and can be used to estimate the compound specific recovery rate of the adsorbents tube.

Despite the discussed limitations of quantitative trace analysis, peak areas can give a sufficient impression of the quantitative relations within one and between different samples. This can be used to understand the formation processes of volatiles (Paczkowski and Schütz, 2011; Kundel et al., 2012) and to assess the practical use of these emissions (Paczkowski et al., 2011a; Paczkowski et al., 2012b).

\section{Electrophysiology}

Odor recognition in insects can be highly compound specific (Vogt and Riddiford, 1981) and can be based on a pattern recognition, as well (Hallem and Carlson, 2006; Bohbot and Dickens, 2012). As the insect's brain or different olfactory receptors were not analyzed in this thesis, no information about pattern recognition was evaluated. However, the information gained by sum potentials of the antenna is sufficient to differentiate between compounds that are detectable or undetectable for insects (Weissbecker et al., 2002; Schütz et al., 1999a; Schütz et al., 1999b; Paczkowski et al., 2012a). 
The fact that insects can perceive a broad variety of compounds suggests that they select the important compounds among the detected compounds by neuronal pattern recognition in higher brain centers. Therefore, the exact role of a volatile, which is electrophysiological active in the GCMS/EAD setup, remains unknown. Additional behavioral studies are necessary to estimate the ecological role of detected volatiles (von Arx et al., 2011; Riolo et al., 2012).

It has further to be considered that the trace analytical method selectively adsorbs volatiles and does not display the complete mixture of VOCs in the natural environment of the insect (Tholl et al., 2006, Paczkowski and Schütz, 2011). Therefore, the GC-MS/EAD method shows only a subset of the complete odor horizon of an insect (Weissbecker et al., 2002). In case of the studies presented here, the subset included the sampled VOCs in a vapor pressure range from $\sim 1$ ppmv (5hydroxymethylfurfural) to $\sim 14,500 \mathrm{ppmv}$ (hexanal) at SATP conditions (Standard Ambient Temperature and Pressure; $25^{\circ} \mathrm{C}$ and $1013 \mathrm{hPa}$ ). Very high vapor pressure compounds like $\mathrm{CO}_{2}$, formaldehyde, or ammonia were not sampled or could not be identified with sufficient quality. It is known that insects can perceive such highly volatile gases for host finding (Garcia et al., 1989). Additional studies, especially on the very high vapor pressure compounds could be performed in order to complete the picture of the odor horizons of the insects investigated in this study.

Both discussed methodical restrictions lead to the conclusion that the GC-MS/EAD results presented in this doctoral thesis are valuable, because they show part of the odor horizon of the investigated insects. These results do not display the relative importance of the odor for the insect and the selectivity of their perception. It is probable that an odor that is perceived by the antenna of the female blowfly is not used by the insect to detect a decaying vertebrate and the information of its presence is eliminated in the higher brain centers (Hannson, 1999; Martel and Boivin, 2011). It is also probable that the insect does not differentiate between dimethyl disulfide, dimethyl trisulfide, and dimethyl tetrasulfide for its orientation towards a cadaver (Hallem and Carlsson, 2006; Stensmyr et al., 2002) or that it perceives $\mathrm{CO}_{2}$ gradients (Garcia et al., 1989) or methanethiol (Lindiger et al., 1998).

The EAG method offers the possibility to evaluate marker compounds (Weissbecker et al., 2004), because this method is used to confirm the electrophysiological response of a compound. Furthermore, the dose-response relation between antenna and compound can be investigated in order to assess the sensitivity of the insect (Paczkowski et al., 2012a). An advanced EAG method even allows the analysis of compound interactions, which further improves the understanding of the odor horizon of insects (Kosanke-Schütz, 2012).

\section{Behavioral studies}

Behavioral studies are a strong tool to confirm electrophysiological results (Aak et al., 2010; von Arx et al., 2011; Martel and Boivin, 2011; Riolo et al., 2012). For each insect investigated in this doctoral thesis, behavioral studies were performed in the laboratory and for $M$. atrata and A. nigricans in the field, as well. Table 1 shows that only three experiments were successful. This indicates that performing a behavioral experiment is not trivial (Martel and Boivin, 2011). An advantage of the electrophysiological studies is the simplification of the natural process of odor recognition. This complex process is reduced to a neuronal response of a receptor population and interpretable as discussed in the section "Electrophysiology". Behavioral experiments do not only evaluate the pattern recognition of the higher brain centers but also the physiology of the complete organism (Peric-Mataruga et al., 2001). The interaction of physiological factors, like nutrition, individual fitness, 
influence of other sensory impressions, influence of hormones, day/night cycles, temperature, and humidity far exceeds our knowledge about insects. The observer of a behavioral study has to keep in mind that a positive reaction towards a perceived stimulus is not necessarily expectable. On the other hand, a repetitive orientation of an insect towards or away from a perceived stimulus in a well designed experimental setup strongly underlines the importance of this stimulus for the insect (Hörmann et al., 2011). However, the design of behavioral studies is challenging, because many factors can distract insects. The position of the light is a very important factor in behavioral experiments, especially for flying species that use the position of the sun for orientation. Many flying insects have an optical sense with a high time resolution, which enables them to see light frequencies not detectable by humans (Agee, 1971). Blowflies are able to perceive the flickering of fluorescent lamps and such lights are usually not suitable for behavioral studies with flying insects. Light emitting diodes are a good alternative to fluorescent lamps, because they do not flicker (Paczkowski et al., 2012c). On the other hand, if the stimulus is extremely interesting for the individual tested, it will orientate towards it despite external irritations (Takasaki et al., 2012). Despite this possibility, behavioral studies require a species specific setup and a large $\mathrm{N}$ number in order to avoid a high standard error or false interpretation (Köhler et al., 2002). The control experiments play an important role for the testing of the setup (Martel and Boivin, 2011). One or several controls (pure solvent, control volatile which is not electrophysiological active or no stimulus) should exhibit no significant orientation. If insects orientate towards one side of a stimulus-free olfactometer with high significance, this could mean that an unknown factors is responsible for this orientation. Behavioral study planning and result interpretation is reviewed and discussed in a paper of Martel and Boivin (Martel and Boivin, 2011). Discussions with the authors led to the interpretation of the behavioral data for A. nigricans and $M$. cuspidata presented in this doctoral thesis.

\section{Statistics}

Besides the principle of using different methodical approaches to verify an information, scientists have another tool for result interpretation, which is statistics. Although statistic consists of different mere mathematical models, which are generally not able to describe complex biological interactions, this tool implies requirements that are valuable for experimental planning and result interpretation. The first statistical requirement is the number of repetitions (Michaud et al., 2012; Köhler et al., 2003). One has to consider that a low number of repetition decreases the exactness of the results by increasing the standard error. A high number of repetitions should be used in order to decrease the influence of chance. On the other hand, a high number of repetitions might have negative implications like time and money consumption and an increasing complexity of the experimental setup that could be harder to interpret than a simple experiment. Therefore, a compromise between a high repetition number and the requirements of the experimental procedure has to be found for each experiment and the results of this experiment have to be interpreted considering this limitation.

Secondly, pseudo-replications have to be avoided (Michaud et al., 2012). If one insect is tested several times, this will increase the repetition number for this insect, not for the species. On the other hand, standardized responses of one insect could be grouped with standardized responses of other insects of the same species in order to analyze both the inner- and inter-individual variation. However, this approach does not increase the number of repetitions on individuals. It is a feasible option when a high number of compounds have to be evaluated on the antennae of insects (screening), but it has to be kept in mind that this decreases the quality of the results and the data 
should be interpreted in that way. One example for this approach is the statistical analysis of the EAG responses of $C$. vicina (Paczkowski et al., 2012a).

Thirdly, an experiment should not test two different characteristics (Michaud et al., 2012). If pyrophilic beetles meet on a charred tree for mating and pheromones are not involved, this will indicate the same olfactory mechanisms for both sexes. Both males and females are attracted by the same compounds and perceive these compounds in the same way (Paczkowski et al., 2012b). In this case, males and females can be grouped for electrophysiological data analysis. In case of the blow flies, almost exclusively females are found on cadavers or meat during field observations and the electrophysiological data for both sexes cannot be grouped, because it is probable that males and females have different olfactory mechanisms (Aak et al., 2010; Paczkowski et al., 2012a). If two characteristics are tested in one experiment and the $\mathrm{N}$ number is large enough, the fitted normal distribution of the data will be bimodal (Köhler et al., 2003).

\section{Discussion of the hypothesis}

The general hypothesis evaluated in this thesis is the utilization of insect olfaction as an information filter. This should decrease the experimental time for trace analytical studies, because the insect's olfaction is adapted to evolutionary selected marker volatiles valuable for applications. This hypothesis was evaluated for the three examples fire detection, post mortem interval estimation, and meat quality control.

The three described methods in chemoecology (trace analysis, electrophysiology and behavioral studies) show the evaluation process of chemoecological data in general and in this thesis. The trace analysis resulted in a set of volatile compounds, which are a selected part of the chemical environment of the insect. Potential marker volatile candidates are selected out of this part by means of electrophysiology and further evaluated by behavioral studies. The applicability of the resulting marker compounds was the most important factor for the verification and this applicability is discussed in the following subchapters.

\section{Fire detection}

All over the world, fire is one of the most important perils and various branches of science contribute to improved fire management. As fires occur in ecosystems since long, the investigation of animals, which are adapted to fires, can be of benefit for fire management. Pyrophilic beetles can detect fires over long distances (Evans, 2010). Their olfaction allows these beetles to smell guajacol derivates (Schütz et al., 1999), which are among the most abundant volatiles in the smoke of a wood fire (Paczkowski et al., 2011). These results suggest that olfaction plays an important role in the orientation of pyrophilic beetles towards forest fires (Evans, 2010).

The aim of the studies on pyrophilic beetles performed during this doctoral thesis was the evaluation of the feasibility of their olfaction for fire warning. Guaiacol is one of the most prominent VOCs in smoke and allows the beetles to detect fires from large distances (Schütz et al., 1999). A more differentiated mechanism has to be used by the beetles for the identification of oviposition places on recently burned areas (Paczkowski et al., 2012b). During the field trips to Australia it has been observed that female and male $M$. atrata accumulated and mated on charred trees with a diameter of a few centimeters (Schmitz, 2011; Personal communication with Prof. Dr. Schütz 2009; Personal observations during field studies in Perth 2010). These trees were usually, but not necessarily, 
positioned close to smoldering logs, which still emitted smoke. The trees had a charred bark; however, they had an intact but heat-damaged cambium. This could be regarded as an optimal place for oviposition. The trees were damaged, but still a source of nutrition. Such trees are usually preferred by bark beetles or wood boring beetles for oviposition (Gilbert et al., 2005). The identification of heated trees on a recently burned area can only be done by volatiles. There is no possibility for the beetle to identify such a tree by means of infrared radiation or by the radiation of the visible spectrum. Only volatiles can show the physiological state of the wood and cambium beneath the charred bark.

The trace analytical results confirm this hypothesis, as there are volatiles typically emitted in an intermediate heating stage between room temperature and ignition. This intermediate stage can be divided into an early intermediate stage, where aldehydes are emitted and a later stage prior to ignition, where furans are emitted. Electrophysiological studies on M. cuspidata and M. atrata performed in this thesis show that the beetles perceive compounds from these groups. The behavioral studies with $M$. cuspidata show a low significant attraction to hexanal and furfural (Paczkowski et al., 2012b). These three experiments allow the conclusion, that hexanal and furfural play a role in the oviposition place finding of $M$. cuspidata and $M$. atrata. On the other hand, both compounds are emitted from other sources, as well (Paczkowski and Schütz, 2011), which seems to be a general inconsistence in the odor perception of insects (Bohbot and Dickens, 2012; Holighaus 2012).

Field studies should be performed in order to prove the attractiveness of hexanal and furfural in a natural environment. The usefulness of these volatiles for applications should be verified by further research. It has not been investigated yet, if a sensor that corresponds to hexanal and furfural will be able to predict a fire in a wood storage. In a BMBF-project the applicability of this bionic sensor concept was evaluated for a wood flake dryer. As the complex air streams in this wood flake dryer led to a mixing of volatiles from heated wood and volatiles from burned bark in the whole system it was only possible to confirm the general feasibility of the concept in a burning chamber experiment (Paczkowski et al., 2012b).

\section{Post mortem interval estimation}

C. vicina is among the most prominent carrion visitors and distributed all over the world. Since it is known that necrophagous insects are present on cadavers in time intervals this can be used for post mortem time estimation in forensic science (Smith, 1986; Amendt et al., 2004). As these insects use their olfaction to find cadavers (Kalinova et al., 2009; LeBlanc and Logan, 2010; Paczkowski et al., 2012a) it is most probable that they use their olfaction for a identification of their time interval in the decaying process (Hoermann et al., 2011; Paczkowski et al., 2012). The aim of the investigation of the olfaction of $C$. vicina was the selection of marker compounds to assess reliably the stage of decomposition. These marker volatiles can serve to narrow the post mortem interval estimation in forensic science, as well.

The results in this study show that $C$. vicina perceives several volatiles emitted by decaying vertebrates. These volatiles show a quantitative dynamic during decomposition of decaying mice (Myodes glareolus) and pig (Sus scrofa domesticus). However, the temperature seems to influence these emissions and, therefore, the onsets of the selected marker compounds' emissions are correlated to a time range rather than to a time point (Michaud and Moreau, 2011, Paczkowski et al., 2012a). This restriction is important for any post mortem interval estimation method. The additional 
value of the volatile based method is the relatively long time range in comparison to established methods (Amendt et al., 2004) and that it is not based on the optical impression of the cadaver.

The identified marker compounds have a strong potential for application, but this potential has to be evaluated further in field studies (Dekeirsschieter et al., 2009; LeBlanc and Logan, 2010; Vass et al., 2002; Statheropoulos and Karma, 2007; Paczkowski et al., 2011; Paczkowski et al., 2012a). The sulfur compounds dimethyl disulfide and dimethyl trisulfide are detected by $C$. vicina at astonishing low concentrations and are emitted in a repeatable and time dependent quantitative dynamic (Paczkowski et al., 2012a). These compounds should be the core of a biomimetik sensor concept for PMI estimation. Aldehydes, phenol, and 1-butanol can give additional information, although the aldehydes show a low emission rate. The most important objection towards this volatile based sensor concept is that $C$. vicina is adapted to the natural process of decay. A volatile pattern influenced by clothing or drug consumption might show an unnatural volatile emission (Boumba, 2008). Furthermore, the studies performed allow a first estimation of the quantitative volatile dynamic of compounds associated with vertebrate decay in deciduous forests in central Europe. It has to be evaluated if the findings are repeatable in this and other ecosystems.

\section{Meat spoilage detection}

In the chain of custody the spoilage of meat cannot always be prevented because of a lack of transparency in the storage conditions (Simons, 2000). The odor perception of $C$. vicina was used to identify marker volatiles of early decay that enable an early detection of meat spoilage and allow an estimation of the potential storage time under given conditions.

The results of the trace analytical experiments show dynamics correlated to storage conditions and storage time. The volatile pattern seems to be dependent on temperature, humidity, and storage time. C. vicina perceives volatiles that can be used for spoilage estimation (Kaib, 1974; Huotari and Mela, 1996; Paczkowski et al., 2012a). However, the fly`s odor horizon is adapted to decaying cadavers in a natural environment and not to spoiled meat pieces stored under artificial conditions. These conditions can be radiation, washing, mincing, vacuum storing, or many other treatments to prevent bacterial growth (Reid et al., 1993; Cheng et al., 2011; Giri et al., 2011). These treatments influence the bacterial growth and in consequence the volatile emission (Paczkowski and Schütz, 2011). Therefore, compounds like 2,3-butanediol are emitted by meat stored under artificial conditions and are not perceived by the fly. In such cases, the information gained by the chemoecological experiments can only be part of the sensor concept. This concept has to be tested in a meat processing facility in order to adapt it to the specific requirements of this facility. First negotiations with Tönnies Fleischwerk GmbH \& Co. KG, Rheda-Wiedenbrück, resulted in discussions on the evaluation of a sensor solution for spoilage detection of freshly butchered pork parts or ground meat, for processed pork meat prior to packaging, or for the assessment of the maturation stage of imported Argentinean beef parts.

\section{General conclusion}

The established methods of Chemoecology applied for this thesis are strong tools for the investigation of insect olfaction. Although the biochemical mechanisms of this olfaction are not completely understood (Hallem and Carlsson, 2006; Bohbot and Dickens, 2012), the functionality of the method is undoubted. However, it has to be considered that the methods have restrictions and these restrictions influence the interpretation of the results. In case of the investigated organisms in 
this study, the ambitious hypotheses of marker compounds serving for industrial applications cannot be completely confirmed. The work performed in this thesis resulted in several very promising marker compound candidates, but the methods used do not allow a complete evaluation of the hypothesis, especially because of the lack of field studies with semi conductor metal oxide sensors.

It remains unknown, if $M$. cuspidata really assesses the heating stage of a stem by temperature dependent volatile emission. Of course, the data in the literature and the own results show a repeatable dynamic in the volatile pattern and these volatiles are perceived by the beetle, but this does not allow a confirmation of the hypotheses. For this confirmation, stems on recently burned areas, which are preferred by $M$. cuspidata, have to be sampled and field behavioral tests have to be performed with odor mixtures resembling the proposed odor profile. The performed sensor tests showed that a sensor shaped for the detection of volatiles emitted prior to inflammation can predict a fire in a burning chamber, which is a first promising step to application.

As $C$. vicina is a generalist among carrion visitors, it is probable that it does not differentiate between all of the stages of decay. However, the marker volatiles perceived by the fly are valuable for PMI estimation. An assessment of habitat induced variation and chemoecological investigations on further necrophagous specialists, as proposed by Matuszewski et al., (Matuszewski et al., 2010) and performed by Hörmann et al., (Hörmann et al., 2011) and Kalinova et al., (Kalinova et al., 2009), will most probably lead to valuable information resulting in a concept that can be evaluated together with forensic staff members of police forces.

The studies on decaying meat gave a further insight into the processes of decay and identified volatiles connected to meat spoilage. This information should be regarded as complementary to other publications on the complicated field of meat spoilage volatiles. The results show that, in case of $C$. vicina, it is not possible to transfer the information gained by chemoecological methods directly to a sensor concept that detects meat spoilage.

The chemoecological studies performed during this doctoral thesis led to results on three selected scientific fields: fire detection, PMI estimation, and meat spoilage detection. Although the benefit of these studies for sensor solutions still has to be evaluated in detail, some results suggest a strong potential for applications. From the point of view of basic science the results are very valuable. Especially the studies on early wood fire detection and PMI estimation in forensic science gave valuable new insights in these fields of science. Therefore, this doctoral thesis is an example for the high potential of the interdisciplinary approach of chemical ecology for both basic science and applied science.

\section{References}

Aak,A., Knudsen,G.K. and Soleng,A. (2010) Wind tunnel behavioural response and field trapping of the blowfly Calliphora vicina. Medical and Veterinary Entomology, 24, 250-257.

Adams,A., Van Lancker,F., De Meulenaer,B., Owczarek-Fendor,A. and De Kimpe,N. (2012) On-fiber furan formation from volatile precursors: A critical example of artefact formation during Solid-Phase Microextraction. Journal of Chromatography B, 897, 37-41.

Agee,H.R. (1971) Flicker fusion frequency of the compound eye of Heliothis zea (Lepidoptera: Noctuidae). Annals of the Entomological Society of America, 64, 942-945. 
Amendt,J., Krettek,R. and Zehner,R. (2004) Forensic entomology. Naturwissenschaften, 91, 51-65.

Andersen,K.B., Hansen,M.J., Feilberg,A. (2012) Minimisation of artefact formation of dimethyl disulphide during sampling and analysis of methanethiol in air using solid sorbent materials. Journal of Chromatography A, 1245, 24-31.

Atkins,P.W. and Jones,L. (2006) Chemie - einfach alles. Wiley-VCH, New York.

Bohbot,J.D. and Dickens,J.C. (2012) Selectivity of odorant receptors in insects. Frontiers in Cellular Neuroscience, doi: 10.3389/fncel.2012.00029.

Boumba,V.A., Ziavrou,K.S. and Vougiouklakis,T. (2008) Biochemical pathways generating postmortem volatile compounds co-detected during forensic ethanol analyses. Forensic Science International, 174, 133-151.

Cheng,A.W., Wan,F.C., Xu,T.C., Du,F.L., Wang,W.L. and Zhu,Q.J. (2011) Effect of irradiation and storage time on lipid oxidation of chilled pork. Radiation Physics and Chemistry, 80:475-480.

Claussen,P.A. and Wolkoff,P. (1997) Degradation products of Tenax TA formed during sampling and thermal desorption analysis: Indicators of reactive species indoors. 31, 715-725.

DeGreef,L.E. and Furton,K.G. (2011) Collection and identification of human remains volatiles by noncontact, dynamic airflow sampling and SPME-GC/MS using various sorbent materials. Analytical and Bioanalytical Chemistry, 401, 1295-1307.

Dekeirsschieter,J., Verheggen,F.J., Gohy,M., Hubrecht,F., Bourguignon,L., Lognay,G. and Haubruge,E. (2009) Cadaveric volatile organic compounds released by decaying pig carcasses (Sus domesticus L.) in different biotopes. Forensic Science International. 189, 46-53.

Evans,W.G. (2010) Reproductive Role of Infrared Radiation Sensors of Melanophila acuminata (Coleoptera: Buprestidae) at Forest Fires. Annals of the Entomological Society of America, 103, 823826.

Garcia,R., Colwell,A.E., Voigt,W.G. and Woodward,D.L. (1989) Fay-Prince trap baited with CO2 for monitoring adult abundance of Aedes sierrensis (Diptera: Culicidae). Journal of Medical Entomology, 26, 327-331.

Giancoli,D.C. (2010) Physik, Lehr- und Übungsbuch. Pearson Studium, München, Germany.

Gilbert,M., Nageleisen,L.M., Franklin,A. and Gregoire,J.C. (2005) Post-storm surveys reveal largescale spatial patterns and influences of site factors, forest structure and diversity in endemic barkbeetle populations. Landscape Ecology, 20:35-49.

Giri,A., Osako,K., Okamoto,A., Okazaki,E. and Ohshima,T. (2011) Effect of meat washing on the development of impact odorants in fish miso prepared from spotted mackerel. Journal of the Science of Food and Agriculture, 91:850-859.

Goll,D.E., Otsuka,Y., Nagainis,P.A., Shannon,J.D., Sathe,S.K. and Muguruma,M. (1983) Role of muscle proteinases in maintenance of muscle integrity and mass. Journal of Food Biochemistry, 7, 137-177.

Hallem,E.A. and Carlson,J.R. (2006) Coding of odors by a receptor repertoire. Cell, 125, 143-160. 
Hansson,B.S. (1999) Insect Olfaction. Springer, New York.

Holighaus,G. (2012) Odour signals relevant to beetles in deadwood habitats - odorants, olfaction and behaviour. Dissertation, Georg-August University Göttingen, Faculty of Forest Science and Forest Protection.

Hudson,N. and Ayoko,G.A. (2008) Odour sampling. 2. Comparison of physical and aerodynamic characteristics of sampling devices: A review. Bioresource Technology, 99, 3993-4007.

Huotari,M. and Mela,M. (1996) Blowfly olfactory biosensor's sensitivity and specificity. Sensors and Actuators B-Chemical, 34:240-244.

Hoermann,C., Ruther,J., Reibe,S., Madea,B. and Ayasse,M. (2011) The importance of carcass volatiles as attractants for the hide beetle Dermestes maculatus (De Geer). Forensic Science International, 212, 173-179.

Hübschmann,H.-J. (2001) Handbook of GC/MS. Wiley-VCH Verlag GmbH, Weinheim.

Jones,M.G. and Chalmers,R.A. (2000) Artefacts in organic acid analysis: occurrence and origin of partially trimethylsilylated 3-hydroxy-3-methyl carboxylic acids. Clinica Chimica Acta, 300, 203-212.

Kaib,M. (1974) Receptors for meat-odor and flower-odor on antenna of blowfly Calliphora-Vicina. Journal of Comparative Physiology, 95:105-121.

Kalinova,B., Podskalska,H., Ruzicka,J. and Hoskovec,M. (2009) Irresistible bouquet of death-how are burying beetles (Coleoptera: Silphidae: Nicrophorus) attracted by carcasses. Naturwissenschaften, 96, 889-899.

Kim,Y.H. and Kim,K.H. (2012) Ultimate detectability of volatile organic compounds: How much further can we reduce their ambient air sample volumes for analysis? Analytical Chemistry, 84, 82848293.

Kosanke-Schütz,K., Gabriel,M., Weissbecker,B., Reinecke,H., Werner,D., Koch,U.T. and Schütz,S. (2012) Sequence matters - selective adaptation in electroantennographic response to binary odour mixtures by the Colorado potato beetle. Journal of Applied Entomology, 135, 372-385.

Kundel,M., Thorenz,U.R., Petersen,J.H., Huang,R.J., Bings,N.H., Hoffmann,T. (2012) Application of mass spectrometric techniques for the trace analysis of short-lived iodine-containing volatiles emitted by seaweed. Analytical and Bioanalytical Chemistry, 402, 3345-3357.

Köhler,W., Schachtel,G. and Voleske,P. (2002) Biostatistik. Springer, Berlin.

LeBlanc,H.N. and Logan,J.G. (2010) Exploiting Insect Olfaction in Forensic Entomology. In Amendt,J., Campobasso,C.P., Goff,M.L. and Grassberger,M. (eds.), Current Concepts in Forensic Entomology. Springer, New York, pp. 205-222.

Lestremau,F., Andersson,F.A.T. and Desauziers,V. (2004) Investigation of artefact formation during analysis of volatile sulphur compounds using solid phase microextraction (SPME). Chromatographia, 59, 607-613.

Lindiger,W., Hansel,A. and Jordan,A. (1998) Proton-transfer-reaction mass spectrometry (PTR-MS): on-line monitoring of volatile organic compounds at pptv levels. Chemical Society Reviews, 27, 347354. 
Maire,F. and Lange,C.M. (2010) Formation of unexpected ions from a first-generation polyamidoamine dendrimer by use of methanol: an artefact due to electrospray emitter corrosion? Rapid Communications in Mass Spectrometry, 24, 995-1000.

Martel,V. and Boivin,G. (2011) Do Choice Tests Really Test Choice. Journal of Insect Behavior, 24, 329-336.

Matuszewski,S., Bajerlein,D., Konwerski,S. and Szpila,K. (2010) Insect succession and carrion decomposition in selected forests of Central Europe. Part 2: Composition and residency patterns of carrion fauna. Forensic Science International, 195, 42-51.

Michaud,J.P. and Moreau,G. (2011) A statistical approach based on accumulated degree-days to predict decomposition-related processes in forensic studies. Journal of Forensic Sciences, 56, 229-232.

Michaud,J.P., Schoenly,K.G. and Moreau,G. (2012) Sampling flies or sampling flaws? Experimental design and inference strength in Forensic Entomology. Journal of Medical Entomology, 49:1-10.

Müller,A., Düchting,P. and Weiler,E.W. (2002) A multiplex GC-MS/MS technique for the sensitive and quantitative single-run analysis of acidic phytohormones and related compounds, and its application to Arabidopsis thaliana. Planta, 216, 44-56

Paczkowski,S. (2008) Masterthesis: Antennal response to decaying meat volatiles and purification of putative $O B P$ in Calliphora vomitoria (Linnaeus, 1758).Department of Forest Zoology and Forest Protection, Georg-August University Göttingen.

Paczkowski,S. and Schütz,S. (2011) Post-mortem volatiles of vertebrate tissue. Applied Microbiology and Biotechnology 91, 917-935.

Paczkowski,S., Sauerwald,T., Weiß.A., Bauer,M., Kohl,C.-D. and Schütz,S. (2011) Biomimetic gas sensors for large-scale drying of wood particles. Martin-Palma, R. J. and Lakhtakia, A. 7975, 797505797505-8. Conference Proceedings SPIE: Bioinspiration, Biomimetics, and Bioreplication, San Diego, USA.

Paczkowski, S., Paczkowska, M., Maibaum, F., and Schütz, S. (2012a) Decaying mouse volatiles perceived by Calliphora vicina Rob.-Desv. Journal of Forensic Sciences. 57, 1497-1506.

Paczkowski,S., Paczkowska,M., Dippel,S., Schulze,N., Schütz,S., Sauerwald,T., Weiß,A., Bauer,M., Gottschald,J. and Kohl,C.-D. (2012b) The olfaction of a fire beetle leads to new concepts for early fire warning systems. Sensors and Actuators B-Chemical, Manuscript submitted and without decision.

Paczkowski,S., Paczkowska,M., Dippel,S. and Schütz,S. (2012c) Volatile combustion products of wood attract Acanthocnemus nigricans (Coleoptera: Acanthocnemidae). Journal of Insect Behavior, Manuscript accepted with major revisions.

Peric-Mataruga,V., Lazarevic,J. and Nenadovic,V. (2001) A possible role for the dorsolateral protocerebral neurosecretory neurons in the trophic adaptations of Lymantria dispar (Lepidoptera: Lymantriidae). European Journal of Entomology, 98, 257-264.

Poliak,M., Fialkov,A.B. and Amirav,A. (2008) Pulsed flow modulation two-dimensional comprehensive gas chromatography-tandem mass spectrometry with supersonic molecular beams. Journal of Chromatography A, 1210, 108-114. 
Reid,D.H., Young,O.A. and Braggins,T.J. (1993) The effects of antioxidative treatments on mutton flavor odor intensity and species flavor differentiation. Meat Science, 35:171-182.

Riolo,P., Minuz,R.L., Anfora,G., Stacconi,M.V.R., Carlin,S., Isidoro,N. and Romani,R. (2012) Perception of host plant volatiles in Hyalesthes obsoletus: Behavior, morphology, and electrophysiology. Journal of Chemical Ecology, 38, 1017-1030.

Schmitz,H. (2011) Personel communication during field studies in Perth, Western Australia, Department of Zoology Friedrich-Wilhelm University Bonn.

Schütz,S., Weissbecker,B., Hummel,H.E., Apel,K.H., Schmitz,H. and Bleckmann,H. (1999a) Insect antenna as a smoke detector. Nature, 398, 298-299.

Schütz,S., Weißbecker,B., Koch,U.T. and Hummel,H.E, (1999b). Detection of volatiles released by diseased potato tubers using a biosensor on the basis of intact insect antennae. Biosensors and Bioelectronics, 14, 221-228.

Simons,J. (2000) Ökonomische Bewertung Regionaler Vermarktungssysteme bei Fleisch, Schriftenreihe Agrarspectrum, 30:130-146.

Smith,K.G.V. (1986) A Manual of Forensic Entomology. Department of Entomology,British Museum (Natural History), London.

Statheropoulos,M. and Karma,S. (2007) Complexity and origin of the smoke components as measured near the flame-front of a real forest fire incident: A case study. Journal of Analytical and Applied Pyrolysis, 78, 430-437.

Statheropoulos,M., Spiliopoulou,C. and Agapiou,A. (2005) A study of volatile organic compounds evolved from the decaying human body. Forensic Science International, 153, 147-155.

Stensmyr,M.C., Urru,I., Collu,I., Celander,M., Hansson,B.S. and Angioy,A.M. (2002) Rotting smell of dead-horse arum florets - These blooms chemically fool flies into pollinating them. Nature. 420, 625626.

Takasaki,T., Namiki,S. and Kanzaki,R. (2012) Use of bilateral information to determine the walking direction during orientation to a pheromone source in the silkmoth Bombyx mori. Journal of Comparative Physiology A, 198, 295-307.

Tholl,D., Boland,W., Hansel,A., Loreto,F., Rose,U.S.R. and Schnitzler,J.P. (2006) Practical approaches to plant volatile analysis. Plant Journal, 45, 540-560.

Tonk,H.Y. and Karasek,F.W. (1984) Quantitation of polycyclic aromatic hydrocarbons in diesel exhaust particulate matter by high-performance liquid chromatography fractionation and highresolution gas chromatography. Analytical Chemistry, 56, 2129-2134.

Vogt,R.G. and Riddiford,L.M. (1981) Pheromone Binding and Inactivation by Moth Antennae. Nature, 293, 161-163.

Von Arx,M., Schmidt-Busser,D. and Guerin,P.M. (2011) Host plant volatiles induce oriented flight behaviour in male European grapevine moths, Lobesia botrana. Journal of Insect Physiology, 57, 1323-1331. 
Vass,A.A., Barshick,S.A., Sega,G., Caton,J., Skeen,J.T., Love,J.C. and Synstelien,J.A. (2002) Decomposition chemistry of human remains: A new methodology for determining the postmortem interval. Journal of Forensic Sciences, 47, 542-553.

Watson,P.R., Van Hove,M.A. and K. Hermann,K. (2004) NIST Surface Structure Database - Version 5.0 National Institute of Standards and Technology. Gaithersburg, MD.

Weissbecker,B., Holighaus,G. and Schütz,S. (2004) Gas chromatography with mass spectrometric and electroantennographic detection: analysis of wood odorants by direct coupling of insect olfaction and mass spectrometry. Journal of Chromatography A, 1056, 209-216.

Wolska,L., Gdaniec-Pietryka,M., Konieczka,P. and Namiesnik,J. (2008) Problems of PAH quantification by GC-MS method using isotope-labelled standards. Talanta, 78, 730-735.

Yoon,S., Nakada,N., Tanaka,H. (2012) A new method for quantifying N-nitrosamines in wastewater samples by gas chromatography-triple quadrupole mass spectrometry. Talanta, 97, 256-261. 


\section{Curriculum Vitae}

Date of Birth:

Born in:

Adress:

Tel.:

E-Mail:

Hochschulprofil:

Family Status:

Nationality:

Oct. 2005 - Apr. 2008

Georg-August-University, Göttingen

Master Forest Science and Forest Ecology

- Final grade 1,5

- Specialization on wood technology and woodbiology, including aspects of chemical analysis and biotechnology

- Master thesis: Antennal response to decaying meat volatiles and purification of a putative odorant binding protein in Calliphora vomitoria (Linnaeus, 1758) (written in English with English speaking supervisor)

Oct. 2002 - Sept. 2005 Georg-August-University, Göttingen

Bachelor Forest Science and Forest Ecology

- $\quad$ Final grade 2,5

Aug. 1998 - July 2002 Infantry regiment Wildeshausen

- Rank: Sergeant

Aug. 1991 - July 1998 Katholic school St. Marien, Berlin Neukölln

- $\quad$ Final grade 2,2 